$\% \%^{\circ} q^{\circ}$ LA-5336

An Engineering Design Siudy of

A Reference Theta-Pinch Reactor (RTPR)

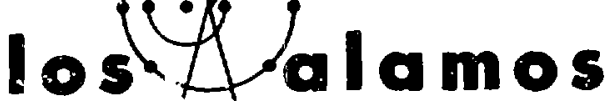

scientific laboratory

of the University of California

los alamos, NeW MEXICO 37544

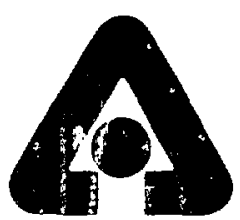

U.JTC. AUA USAEC

ARGONNE NATIONAL LABORATORY

ARGONNE, ILLINOIS 
This report was prepared as an account of work sponsored by the United Stntes Government. Neither the United States nor the United States Atomic Energy Commission, nor any of their employees, nor any of their contractors, subcontractors, or their employees, makes any warranty, express or implied, or assumes any legal liability or responsibitity for the accuracy, completeness or usefulness of any information, apparatus, product or process disclosed, or represents that its use would not infringe privately owned rights.

Printed in the United States of America. Availabie from National Technical Information Service

U. S. Department of Commerce

5285 Port Royal Road

Springfield, Virginia 22151

Price: Printed Copy $\$ 7.60$ Microfiche $\$ 1.45$ 


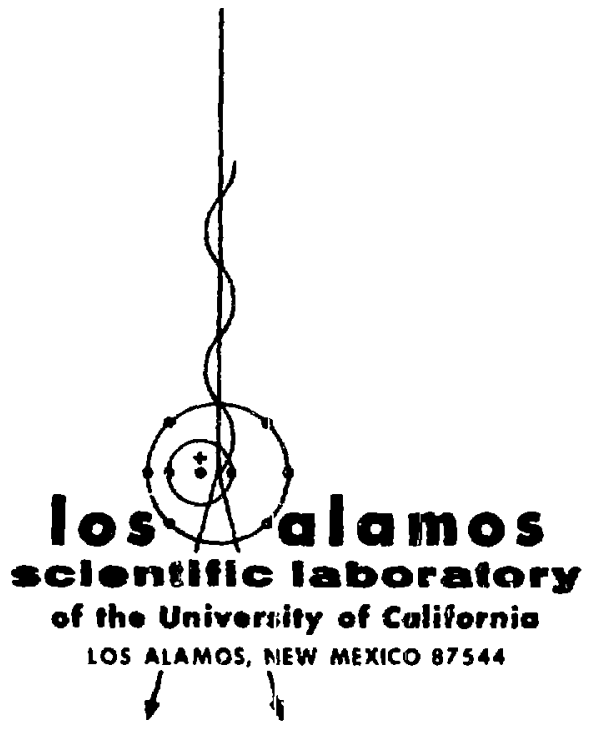

\author{
LA. 5336 \\ ANL-8019 \\ SPECIAL DISTRIBUTION \\ ISSUED: March 1974
}

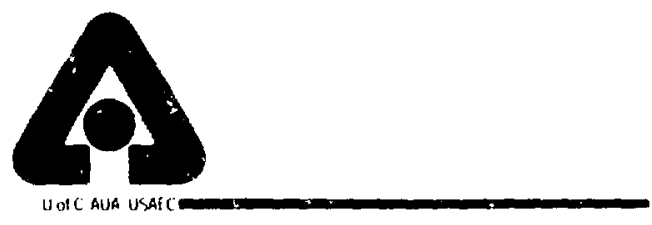

ARGONNE NATIONAL LABORATORY ARGONNE, ILLINOIS

\title{
An Engineering Design Study of A Reference Theta-Pinch Reactor (RTPR)
}

\author{
A Joint Report
}

Prepored for the U.S. Atomic Energy Commission

by

Argonne National Laboratory and

Los Alamos Scientific Laboratory

This report was prepsred as an account of work
sponsored by the United States Government. Neither
the United States nor the Untited States Atomic Energy
Commission, nor any of their employees, nor any of
the ir contractors, subcontractors, or their employees,
makes any warranty, express or implied, or assumes any
legal liability or responsibility for the accuracy, com-
pleteness or usefulness of any information, apparatus,
product or process disclosed, or represtents that its use
would not infringe privately owned rights. 


\section{CONTENTS}

Foreword and Acknowledgments . . . . . . . . . . . . . . . . . . v

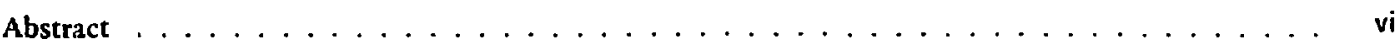

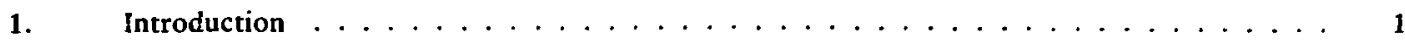

1.1 Background and Review of CTR Concepts . . . . . . . . . . . . . . 1

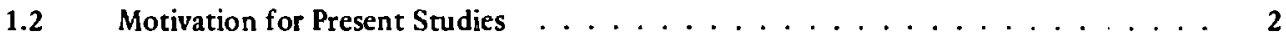

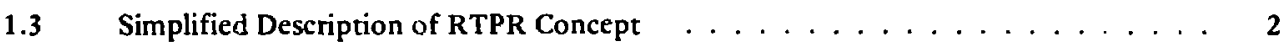

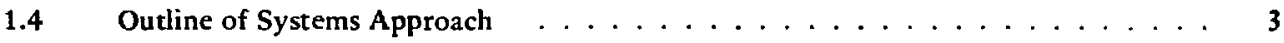

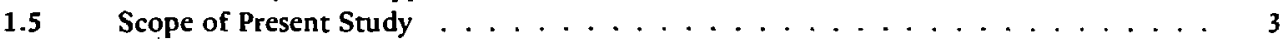

2. Basic Concepts of the Staged Theta-Pinch System . . . . . . . . . . . . . . 5

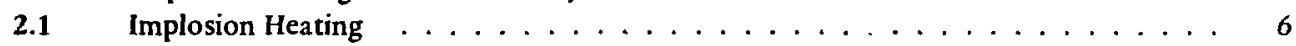

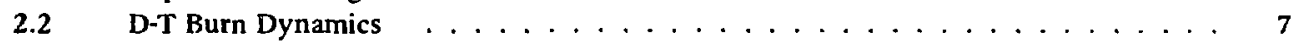

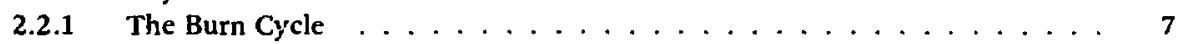

2.2.2 The Direct Conversion Cycle . . . . . . . . . . . . . . . . . . . . . 9

2.3 Plasma Cooling by Neutral Gas Layer . . . . . . . . . . . . . . . 10

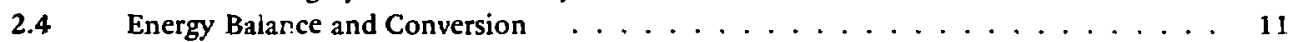

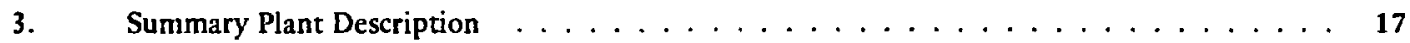

3.1 Overall Plant Layout . . . . . . . . . . . . . . . . . . . . 17

3.2 Description of the Reactor Module . . . . . . . . . . . . . . . . 17

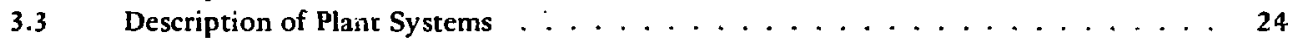

3.3.1 Implosion Heating System . . . . . . . . . . . . . . . . 24

3.3.2 Adiabatic Compression Coil System . . . . . . . . . . . . . 25

3.3.3 Magnetic Energy Transfer System . . . . . . . . . . . . . 25

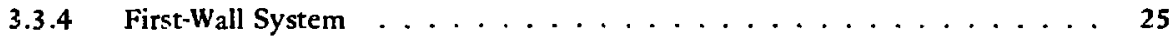

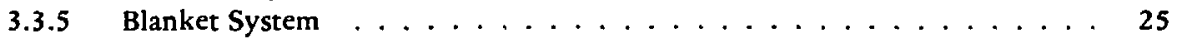

3.3.6 Biological Shielding System ... . . . . . . . . . . . . 25

3.3.7 Fuel Supply and Removal System _................. 26

3.3.8 Fuel Processing System ..................... 26

3.3.9 Coolant Processing System . . . . . . . . . . . . . . . . 26

3.3.10 Energy Conversion System . . . . . . . . . . . . . 26

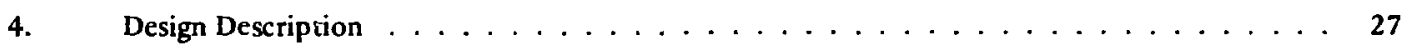

4.1 Implosion Heating System . . . . . . . . . . . . . . . . . . . . 27

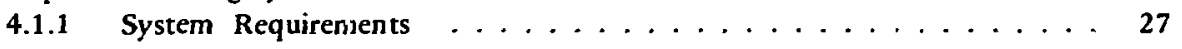

4.1.2 Physics of the Implosion Heating Process . . . . . . . . . . . . . . 27

4.1.3 Design Aspects of Implosion Heating System . . . . . . . . . . . . 28

4.1.3.1 Electrical Interaction of Implosion Hearing

Coil with Other Systems ................ 28

4.1.3.2 Construction of Implosion Heating System . . . . . . . . . . 29 
Plasma Confinement and Compression System $\ldots \ldots \ldots \ldots$. . . . . . . 30

4.2.1 System Requirements . . . . . . . . . . . . . . . . . 30

4.2 .2 Physics of Adiabatic Compression $\ldots \ldots \ldots \ldots \ldots \ldots \ldots$

4.2 .3 Compression Coil Design ................. 31

4.2.3.1 Electrical Design of Compression Coil . . . . . . . . 31

4.2.3.2 Radiation Heating in the Compression Coil . . . . . . . 32

4.2.3.3 Mechanical Stresses in the Compression Coil ........ 33

4.2.3.4 Mechanical Design . . . . . . . . . . . . . . 33

4.3 Magnetic Energy Transfer and Storage System . . . . . . . . . . . . 34

4.3 .1 System Requirements . . . . . . . . . . . . . . . . 34

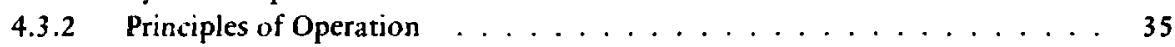

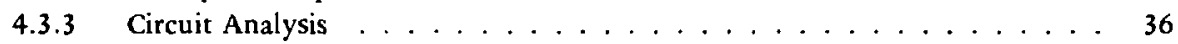

4.3 .4 Design Features . . . . . . . . . . . . . . . . . . 37

4.3.5 Switching System $\ldots \ldots \ldots \ldots \ldots \ldots \ldots$

4.3 .6 A C Losses and Heat Load . . . . . . . . . . . . . . . 41

4.3.7 Concluding Remarks on METS System . . . . . . . . . . 41

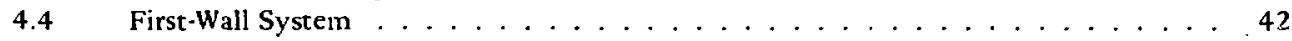

4.4 .1 System Requirements . . . . . . . . . . . . . . . 42

4.4 .2 The First-Wall Insulator . . . . . . . . . . . . . . 44

4.4 .3 The First-Wall Metal $\ldots \ldots \ldots \ldots \ldots . \ldots . \ldots . \ldots 44$

4.4 .4 Heat Transfer ai the First Wall $\ldots \ldots \ldots \ldots \ldots \ldots$

4.4.5 First-Wail Stress Calculations . . . . . . . . . . . . . 48

4.4.6 Failure Modes for the First Wall . . . . . . . . . . . . 50

4.4.6.1 First-Wall Failure Induced by Excessive

Temperature ................ 50

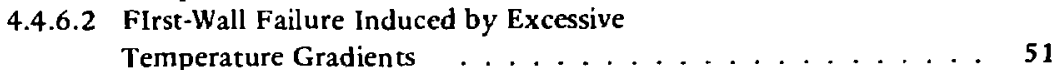

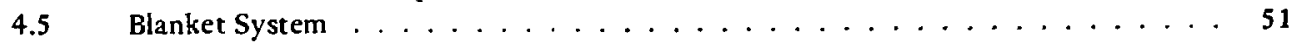

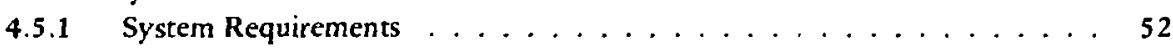

4.5 .2 Neutronics . . . . . . . . . . . . . . . 52

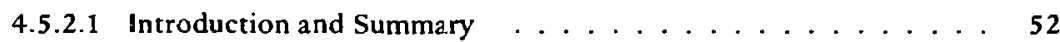

4.5.2.2 Neutronic Model $\quad \ldots \ldots \ldots \ldots . \ldots . \ldots 54$

4.5.2.3 Analysis of Blanket Reaction Rates . . . . . . . . . . 57

4.5.2.4 Induced Radioactivity . . . . . . . . . . . . . . . 63

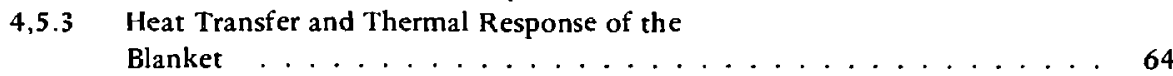

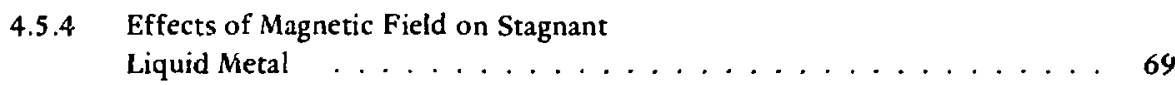

4.5.5 Lithium Flow Transients within Blanket . . . . . . . . . . . 71

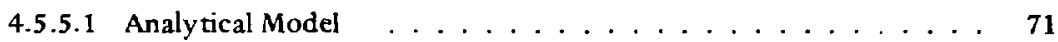

4.5.5.2 Results and Conclusions . . . . . . . . . . . . 72

4.5.6 Blanket Component Interactions with Forces Other $\ldots \ldots \ldots \ldots \ldots \ldots$

4.5.6.1 Forces Related to Pressure Differentials . . . . . . . . . 73

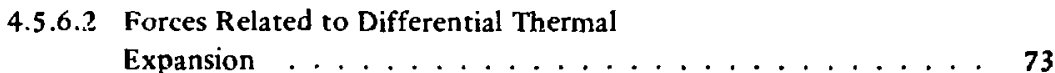

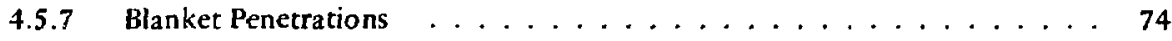

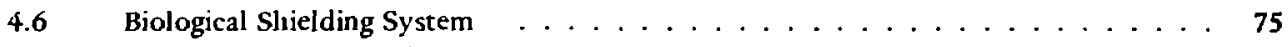

4.6.1 System Requirements . . . . . . . . . . . . . . 75

4.6.2 General Shielding Engineering Layout $\ldots \ldots \ldots \ldots \ldots$

4.6.2.1 Reactor Core Components . . . . . . . . . . . . . . . 75

4.6.2.2 Controlled and Noncontrolled Areas . . . . . . . . 75

4.6.2.3 Shield Design and Composition . . . . . . . . . . 76

4.4 .2 .4 Shielding of Coolant System . . . . . . . . . . 77

$4.7 \quad$ Fuel Supply and Removal System $\ldots \ldots \ldots \ldots \ldots \ldots$ 
4.7.1 System Requirements . . . . . . . . . . . . . . . . . 78

4.7.2 Physical Principles . . . . . . . . . . . . . . . . 78

4.7.3 Design of Fueling System . . . . . . . . . . . . . . . . 79

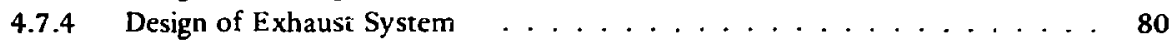

4.7.5 Leakage Through the Walls of the Toroid . . . . . . . . . . . 82

4.8 Fuel-Ash Processing System _. . . . . . . . . . . . . . . . 82

4.8.1 System Requirements . . . . . . . . . . . . . . . . 82

4.8.2 Mcthods to Separate Protium and Helium . . . . . . . . . . . 82

4.8.3 Design Description of Cryogenic Distillation

System ...................... 82

$4.9 \quad$ Lithium Processing System . . . . . . . . . . . . . . . . . . 84

4.9 .1 System Requirements .................... 84

4.9.2 Method of Recovering Tritium from Lithium . . . . . . . . . . . . . . 84

4.9.2.1 The Behavior of Tritium in Liquid

Metals .................... 84

4.9.2.2 Tritium Management and Recovery Schemes . . . . . . . . 85

4.9.3 Molten Salt Contactor . . . . . . . . . . . . . . . . . 85

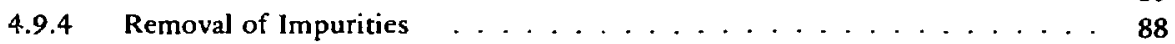

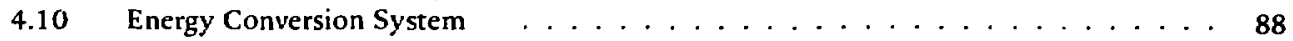

4.10 .1 System Requirements . . . . . . . . . . . . . . . . . 88

4.10 .2 Blanket Cooling Systems . . . . . . . . . . . . . . . . 88

4.10.2.1 General Description ................... 89

4.10.2.2 Intermediate Heat Exchanger and Intermediate

Heat Transfer Loop . . . . . . . . . . . . . 89

4.10 .3 Power Plant . . . . . . . . . . . . . . . . . . . . 89

4.10.3.1 Conventiona! Sodium Steam Power Plant . . . . . . . . . . . 89

4.10.3.2 Advanced Potassium Topping Cycle Power

Plant ..................... 89

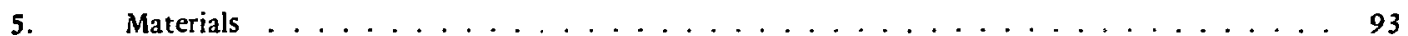

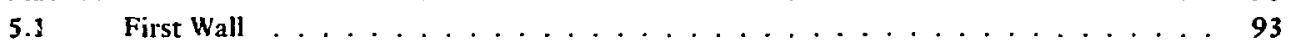

5.1.1 First-Wall Insulator ..................... 93

5.1.1.1 Effects of High Temperature on Electrical and

Structural Properties .................. 94

5.1.1.2 Effects of Neutron Bombardment on Electrical and Structural Properties . . . . . . . . . . . . . 94

5.1.1.3 Effecss of Bremsstrahlung and Gamma Irradiation . . . . . . . 96

5.1 .2 First-Wall Metal . . . . . . . . . . . . . . . . 96

5.1.2.1 Effects of Cyclic Stress and Fatigue on Strength ..................... 96

5.1.2.2 Radiation Effects on First-Wall Metal . . . . . . . . . . 97

5.1.2.3 Chemical Interactions with First Wall . . . . . . . . . . 99

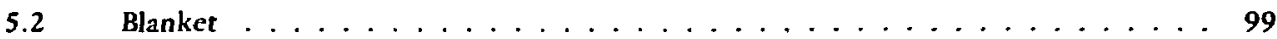

5.2.1 Beryllium Region ...................... 100

5.2 .2 Graphite Region . . . . . . . . . . . . . . . . 100

5.2.3 Structural Metals in Blanket .................. . . . . . . . . . . . . . . .

5.3 Compression Coil ......................... 100

5.4 Coolant ............................ 101

5.4.1 General Problems with Lithium . . . . . . . . . . . . . . . 101

5.4 Corrosion Effects by Lithium . . . . . . . . . . . . . . . . 101

5.4.2.1 Corrosion of Refractory Metals . . . . . . . . . . . . . 101

5.4.2.2 Corrosion of Ceramic Materials . . . . . . . . . . . . 101

5.4.2.3 Corrosion of Graphize . . . . . . . . . . . . . 104 


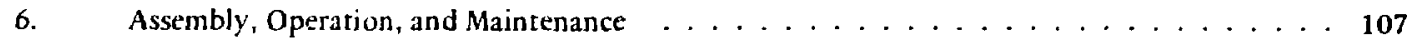

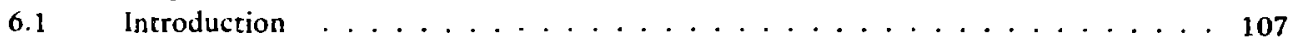

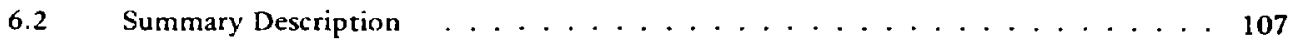

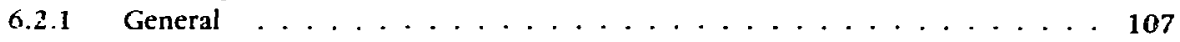

6.2 .2 Consiruction and Assembly of a Module . . . . . . . . . . . . 107

6.3 Module Handling and Maintenance . . . . . . . . . . . . . . 108

6.3 .1 Module Lifetime . . . . . . . . . . . . . . . . 108

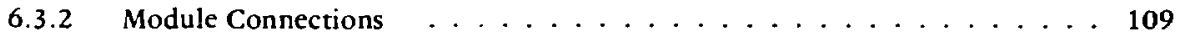

6.3 .3 Module Failure Modes ... . . . . . . . . . . . . . 109

6.3 .4 Module Replacement . . . . . . . . . . . . . . . . 109

6.3 .5 Module Storage and Repair . . . . . . . . . . . . . . . . . 109

6.4 Miscellaneous Startup and Maintenance Problems . . . . . . . . . . . . 110

Appendix A. Derivation of Design Relationships for the Implosion

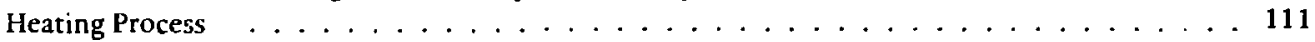

A.1 Simple Implosion Heating Mod $\iota_{1} \ldots \ldots \ldots \ldots \ldots \ldots \ldots$

A.2 Programmed Implosion Heating; Free Expansion

of lons ............................... 112

Appendix B. Computer Output Summary of RTPR Parameter Design

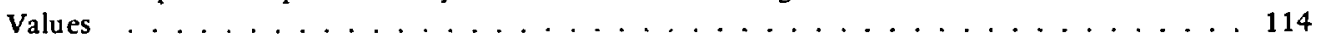

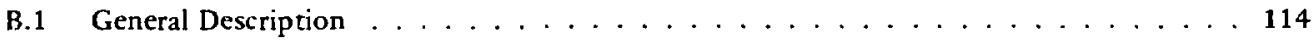

B.2 Potential of System . . . . . . . . . . . . . . . . . . . . 114

B.3 Description of Existing RTPR System . . . . . . . . . . . . . . . . 114

B.4 Design Data for Reference Theta Pinch Reactor (RTPR) . . . . . . . . . . 115

Appendix C. Effects of the Magnetic Field on Stagnant Liquid Metal . . . . . . . . . . . . 128

C.1 Effects on Fluid in Flow Channels Parallel
to the Field . . . . . . . . . . . . . . . . . . . . . 128

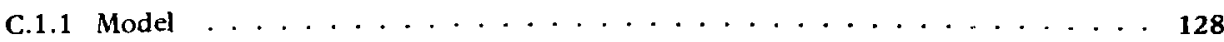

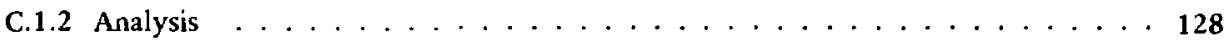

C.1.3 Numerical Results . . . . . . . . . . . . . . . . . . . . 130

C.2 Effects on the Fluid in the Feed Lines Perpendicular

C.2.1 Model ............................ 130

C.2.2 Analysis ............................131

C.2.3 Numerical Results . . . . . . . . . . . . . . . . . . . . 132

C.3 Effects of Magnetic Field on Flow Channels . . . . . . . . . . . . . . . 132

C.3.1 Model ........................... 132

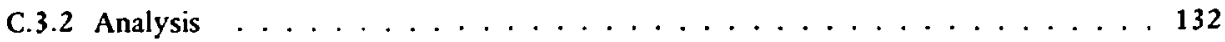

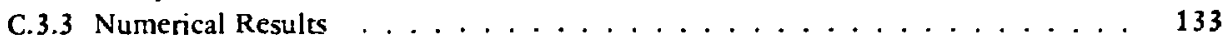

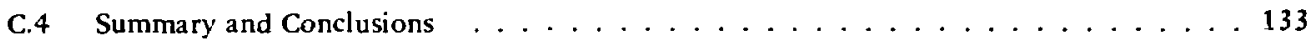

Ajpendix D. Mechanical and Eiectrical Aspects of a Homopolar Motor

Generator as a Trarisfer Capacitor . . . . . . . . . . . . . . . . . . 134

D.1 Homopolar Motor Generator . . . . . . . . . . . . . . . . . . . . 134

D.2 Homopalar Capacitor ........................... 134

D.3 Rotor Speed and Material . . . . . . . . . . . . . . . . . . . . . 134

D.4 Th. Use of a Homopolar Capacitor ...................... 135

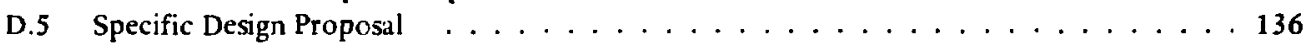




\section{FOREWORD AND ACKNOW LEDGMENTS}

The conceptual design of a controlled thermonuclear reactor (CTK) based on the the ta-pinch principle began in the mid-1960s. These early efforts were directed primarily toward establishing physics requirements of the pulsed theta-pinch concept. This report, Volume $l$ of a three-volume series on engineering design of the Reference Theta-Pinch Reactor (RTPR), assembles the physics and engineering aspects of the RTPR into a reasonably selfconsistent systems aralysis. This report also provides sufficient engineering detail to allow realistic assessments of the environmental impact of the RTPR and of the rechnological problems envisioned in its development, cunsituction, and operation. Volume 11 investigates the environmental impact of the RTPR, and Volum: Ill deals with major unresolved technological problems.

At the present evolutionary stage of the RTPR design, it should come as no surprise that a number of design problems are unresolved. Their resolution will depend on new knowledge as well as on trade-offs between design requirements and functions of the various RTPR systems.
Recognition of these problems and awareness of inconsistencies which they may entail is of utmost importance in realizing the benefits of fusion energy. Special emphasis has been given to elucidating design inconsistencies, identifying technolcgical problems, and presenting (when possible) viable and realistic solutions.

This report is a compilation of efforts by many people at the Argonne National Laboratory (ANL) and the Los Alamos Scientific Laboratory (LASL). Major contributors are listed below and are identified at the beginning of their respective section(s). The editors wish to express their appreciation to the staff at ANL and LASL who contributed by comment or coraposition to this document.
R. A. Krakowksi, F. L. Ribe (LASL) T. A. Coultas, A. J. Hatch (ANL)

July 1, 1973

\section{CONTRIBUTORS}

LASL
J. L. Anderson
M. Bowman
F. W. Clinard
D. J. Dudziak
W. V. Green
R. A. Krakowski
J. Marshall
T. A. Oliphant
F. L. Ribe
K. I. Thomassen

AN L

L. J. Anastasia

T. Bump

R. J. Burke

J. Cook

T. A. Coultas

p. Dauzvardis

C. B. Dennis

S. A. W. Gerstl

A. J. Hatch

D. C. Hess

V. A. Maroni

P. J. Persiani

M. Potrick

E. Pierson

A. T. Santhanam

J. H. Talboy 


\begin{abstract}
This report describes an engineering conceptua! design study of a fusion reactor power plant based on the theta-pinch concept. A high-beta, deuteriumtritium (DT) plasma is produced by means of a programmed pulsed magneric field in an approximately toroidal chamber having major and minor radii of 56 and 0.5 meters, respectively. The field pror ices a short, fast implosion stage followed by a longer adiabatic compression stage during which the DT fusion occurs. The DT burn stage has a duration of $\sim 0.080 \mathrm{sec}$, and the cycle time is $10 \mathrm{sec}$. To protect the first wall after the burn, a gas blanket is used to cool the plasma in a slowly' decreasing magnetic field. The fusion reactor power plant consists of 10 major systems as follows: implosion heating, plasma compression and confinement, magnetic energy transfer and storage, first wall, blanket, biological shield, fuel supply and removal, fuel-ash reprocessing, lithium processing, and energy conversion. Liquid lithium is the primary coolant. Other materials include alumina, provisionally chosen as the first-wall insulator, niobium as the first-wall and blanket structural material, beryllium as the neutron breeder, and graphite as a moderator. Two energy conversion schemes are considered: i) a conventional, low-temperature system which uses a $\mathrm{Na}$ steam cycle, and ii) an advanced, high-temperature steam system which uses a potassium topping cycle. Special attention is given to plasma burn dynamics, overall energy balance, blanket neutronics and thermal response, radiation shielding, radiation effects on and corrosion of construction materials, thermal and irradiation response of the $\mathrm{Al}_{2} \mathrm{O}_{3}-\mathrm{Nb}$ first wall, and tritium recovery and containment. Unique features of the design include a novel superconducting magnetic energy transfer and storage system and a modular blanket siructure which permits easy access for maintenance. The engineering design is detailed enough to provide the basis for an environmental impact study (Vol. II) and an assessment of future research and development (Vol. III).
\end{abstract}




\section{INTRODUCTION*}

This first comprehensive design of a fusion reactor power plant based on the theta-pinch concept of plasma heating and confinement is the culmination of two major phases of study. The first phase was a seri zs of preliminary feasibility studies of various theta-pinch designs begun in 1965 at the Los Alamos Scientific Laboratory (LASL) and culminating in a design study for a Reference Theta-Pinch Reactor (RTPR) in 1972. The secund phase was a more comprehensive design study carried out jointly by LASL and the Argonne National Labotatory (ANL) during 1972-73. The present design represents the first iteration in a series of comprehensive conceptual designs planned to be carried out during the 1970s.

This report presents a self-consistent engineering plan for a fusion reactor based on the theta-pinch concept, with elucidation of major technological problems as a minimum goal. Proposed solutions for some of these problems are given whenever the associated technology is sufficiently advanced. Some of the engineering problems intrinsic to the RTPR, however, are not solved here, and some of the proposed solutions in fact need considerable development to become workable. The authors clearly recognize this situation. Nonetheless, an unambiguous and realistic assessment of the technology required to construct and operate a theta-pinch power plant is attempted; the present and future modus operandi required to achieve engineering feasibility should be more lucidly defined by successive design iterations.

This chapter sets the stage for the remainder of the report by reviewing the background of previous design studies, establishing the motivation for the present design, providing a simplified description of the theta-pinch concept, summarizing the ground rules adopted for the present design, and outlining the systems methodology employed in the design.

\subsection{BACKGROUND AND REVIEW CF CTR CON- CEPTS}

The earliest feasibility studies of pulsed theta-pinch fusion reactors ${ }^{1-3}$ deale with systems in which a singleturn coil furnished magnetic fields for both the implosion

*R. A. Krakowski, F. L. Ribe, LASL.: T. A. Coultas, A. J. Hatch, ANL. heating and adiabatic compression, as has been the case in most theta-pinch plasma experiments to dare. In these early studies the magnet coil was situated inside the neutron blanket and was gas-cooled. Such a system presented difficulties having to do with materials properties of the compression coil and with obtaining a sufficiently large excess of reactor energy output over the joule losses incurred in producing and maintaining the magnetic field.

Tc overcome these difficulties, a staged, thet 1 -pinch syscem $^{4-7}$ was developed in which separate energy sources were used for the implosion-heating and adiabatic-compression stages. The initial implosionheating field was provided by a relatively low-energy, high-voltage circuit discharging in a few microseconds through a thin coil cooled by a liquid metal. The subsequent adiabatic-compression field was provided by a high-energy, low-voltage circuit which established a more slowly sising magnetic field appropriate to adiabatic compression of the implosion-heated plasma; the compression field was maintained for tens of milliseconds, and the multiturn compression coil was located uutside the neitron moderating blanket and was gas-cooled (helium). This design was shown to be economical of joule electrical losses and, therefore, was capable of yielding a satisfactory excess of reactor power output.

Th: feasibility studies performed heretofore have focused on the plasma prucesses and included detailed time-dependent computations of the effects of D-T burnup and alpha-particle production on plasma parameters such as net power production, bremsstrahlung losses, and direct conversion. Past feasibility scudies also considered several of the most prominent engineering and technological aspects of a contro'!ed chermonuclear reactor (CTR) such as blanket neutronics, tritium breeding, energy conversion, heat transport, and radiation damage. The most advanced and comprehensive of these srudies was for the Reference Theta Pinch Reactor ${ }^{7,8}$ (RTPR) based on the staged theta-pinch concept and operated as a pulsed system.

The staged, theta-pinch concept represents the point $c f$ departure for the present design, although the computations of plasma parameters have been markedly improved and refined. ine major thrust of the present design, however, is in the direction of a greatly expanded scope 
of engineering and technolugical requirements of the design. To the extent that all essential physicial systems and subsysteris have been included, the present work represents the first somplete, full-scale design of a pulsed theta-pinch fusion reactor. Furthermore, the study has been extended to include the first realistic assessment of the environmental impact of the present specific design. Finally, the design has provided the basis for an evaluation of critical design problems and for an assessment of the near-term and long-range research and development efforts required to bring the RTPR to fr:ition. The latter two topies are included undar separate cover (Volumes II and III, respectively).

\subsection{MOTIVATION FOR PRESENT STUDIES}

The earlier studies of the RTPR performed by LASL were undertaken to explore the applicability of the thetapinch concept to an actual fusion reactor. The increased emphasis on feasibility studies for all fusion reactor concepts in 1968-69 was motivated by the well-publicized improvements in plasma confinement and heating that occurred about that time; fusion reactor technology has becime an essential activity in the major fusion efforts since the breakthrough in plasma physics which occurred in the late $1960 \mathrm{~s}$.

The present LASL-ANL design has an immediate and specific purpose of defining a realistic engineering design on which to base an environmental impact assessment for presentation at an international conference on this subject in Jan. 1974. The RTPR design as well as designs for other fusion concepts will provide the basis of the first realistic assessment of the oft-proclaimed envirommental advantages of fusion power. Feedback to the plasma research program represents a more general purpose of this and futur: studies.

Ihis study also establishes the research and development requirements of a technology program necessary for an orderly transition from the present research-dominated fusion program to an engineering-domunated program. This transition is expected in about 1978-80 if hopes for the demonstration of scientific feasibility of fusion are realized. Experience in the various fission-reactor programs has shown that lead times of about 5 to 10 years for such technological build-up is not unreasonable and in some cases have proved to be too short.

\subsection{SIMPLIFIED DESCRIPTION OF RTPR CONCEPT}

The theta-pinch plasma is produced in a toroidal chamber having a minor radius of $0.5 \mathrm{~m}$ and a major radius of $56 \mathrm{~m}$. Surrounding the plasma chamber is a $0.4 \mathrm{~m} \mathrm{multi-}$ region blanket composed of lithium, beryllium, and graphite. The blanker composition is optimized to obtain maximum tritium breeding while assuring adequate neutron thermalization, cooling, and radiation shielding for a minimized thickness. The implosion heating coil is situated immediately outside the blanket. The adiabatic compression coll ( $\sim 0.4 \mathrm{~m}$ thick) surrounds the implosion coil. Both coils are thermally as well as electrically isolated. A biological radiation shield surrounds the core (plasma, blanket, implosion, and adiabatic compression coil) region.

The pulsed cycle starts with a U-T thermonuclear burn time of nominally $0.080 \mathrm{sec}$. The bulk of the cycle is used to cool the post burr plasma which contains about 5\% alpha-particle "ash"), remove the burn products from the plasma chamber, and refuel the reactor with a 50:50 mixture of D-T. The kinetic energy of the (14.1 MeV) neutrons plus the energy associated with biemsstrahlung and gamma radiations are converted to thermal energy in the lithium-beryllium-graphite blanket. This thermal energy is transported by liquid lithium to the energy conversion system. Two (alternative) thermal conversion systems have been designed. One design uses a conservative steam plant which is typical of present power plants; the other design proposes an advanced steam plant with a potassium topping cycle. An important energy bonus results from work done during t.e D-T burn by the expanding hot plasma against the confining magnetic rield. This direcr conversion energy is returned directly to the magnetic energy transfer system (METS) used for the adiabatic compression heating and makes up most of the losses incurred within that system.

The toroidal plasna chamber, blanket, shielding, primary heat exchanger, and related systems (vacuum pumps, tritium recovery, fuel preparation, coolant pumps, etc.) constitute the "nuclear island" of the fusion power plant. The intermediate heat exchangers, steam generators, turbogenerator, and other reiated systems outside this nuclear island represent standard items and are not treated in detail by this study. Much of the developing LMFBR power conversion technology can be applied directly to the low-temperature option of the RTPR design, whereas considerable development is required for the more advanced, high-temperature system.

The gross thermal power developed by the $R$.PR is $3600 \mathrm{MWt}$, which is based upon a conservative cycle time of 10 seconds per pulse. The resulting first-wal! loading is $2.0 \mathrm{MW} / \mathrm{m}^{2}$ (14.1 MeV neutron curtent) and comparable to wall loadings for other fusion power plants. This value is dictated by estimated radiation damage effects to structural and first-wall materials. Should the damage effects prove less severe than presently envisioned, other limitations would allow duty cycle times as low as three seconds. In portions of this report where the three second cycle time poses more severe design requirements it is used in exemplary numerical calculations rather than the less stringent 10 second time. Using the advanced potassium topping cycle, the on-line power from the plant is 
$1700 \mathrm{MWe}$. In any event, the adopted values of cycle time, plant efficiency, and on-line output power are respectively $\tau_{c}=10 \mathrm{sec}, \eta_{\mathrm{TH}}=0.564$, and $\mathrm{P}_{\mathrm{E}}=1700 \mathrm{MWe}$.

\subsection{OUTLINE OF SYSTEMS APPROACH}

At the most fundamental level the RTPR can be considered as comprising two major systems, (1) the plasma system, which is the prime source of thermonuclear energy, and (2) other systems that provide the means for establishing the plasma and utilizing its energy.

Plasma processes in the theta-pinch concept in rlude implosion (shock) heating, adiabatic compression, thermonuclear burn dynamics, energy production, and plasma cooling dynamics. These processes establish the reactor parameters and requirements. Because of the basic nature of these processes and the extensive analytical and numerical treatment involved, the plasma system and energy balance are discussed and reviewed in Chapter 2. The engineering system which utilizes the basic principles outlined in Chapter 2 is described in Chapter 3. This chapter is devoted entirely to the engineering and plant design of the RTPR.

The systems described in Chapter 3 must interface with a situation determined in large part by the plasma processes and are subject to constraints imposed by magnetoelectrodynamics, heat transfer, and fluid dynamics. Additionally the physical properties of materials, such as neutronic and radiative properties, resistance to radiation damage, electrical and thermal conductivitics, thermal capacity, working temperatures, structural properties, and chemical behavior and compatibility, dictate real technological limitations on the overall reactor design. To facilitate a logical, manageable, and self-consistent approach to the engineering design of RTPR, a systems approach has been adopted.

The systems ritethod entails division of the reactor into a set of systems each of which has a well-defined major function and interfaces distinctively with other systems. Following this design philosophy, the RTPR has been devided into ten systems as follows:

- Implosion Heating System

- Plasma Confinement and Compression System

- Magnetic Energy Transfer and Storage System

- Hirst-Wall System

- Blanket System

- Biological Shield System

- Fuel-Ash Supply and Removal System

- Fuel Processing System

- Coolant Processing System

- Finergy Conversion System

In most cases this division was made along functional lines, the most obvious exception being the first-wall system. Although the first wall might be considered as merely the innermost edge ot the blanket system, its functional role of plasma-reactor interface makes it one of the more crucial and interesting problem areas of the design of any fusion reactor. Therefore, for the purposes of the present design the first wall is considered as a separate system. A detailed analysis and design of each of these ten reactor systems and the way in which each system interfaces with each other is given in Chapter 4.

\subsection{SCOPE OF PRESENT STUDY}

An ideal design is one in which all paraneters and constraints are incorporated into a mathematical functional dependence capabie of being manipulated computationally to determine a unique set of parameters, optimized with respect to some desired criterion. Such an optimization seems premature. Instead, the design described below represents only an early ireration on many possible sets of parameters. The necessity of making compromises and trade-offs to obtain a mutually selfconsistent set of design parameters, however, has resulted in a first-order design optimization. New technical and economic information will be required before a reasonably clear optimum can be attained.

In addition to the discussion of basic plasma processes (Chapter 2), the RTPR power plant (Chapter 3), and the RTPR systems (Chapter 4), Chapter 5 is devoted exclus ively to the materials problems expected in and imposed on the RTPR design described in previous parts of the report. In some cases the RTPR design reported herein is pushing material requirements up to and beyond the present state of the art; undoubtedly either future materials developments or relaxation of specific design requirements will be required.

Lastly, the method by winich the proposed RTPR design will be fabricated, operated, and repaired is described in Chapter 6. Once again the proposals made in Chapter 6 represent a first iterarion, although a reasonable amount of consistency exists between plasma requirements, system requirements, material requirements and the fabrication, operation, and maintenance requirements.

\section{REFERENCES}

1. F. L. Ribe, T. A. Oliphant. Jr., and W. E. Quinn, "Feasibility Study of a Pulsed Thermonuclear Reactor," Los Alamos Scientific Laboratory Report LA-3294 MS (May 1965).

2. G. I. Bell, W. H. Borkenhagen, and F. L. Ribe, "Feasibility Studies of Pulsed, High $\beta$ Fusion Reactors," in Proceedings of the B.N.F.S. Conference on Nuclear Fnsion Reactors UKAEA Culbam Laboratory, Sept. 17-19, 1969. pp. 242-254.

3. F. L. Ribe, "Economic Considerations and Magnetic Energy Storage for High- $\beta$, Pulsed Reactors," in Fusion Technology. Proceedings of the Intersociety Energy Conversion Engineering Conference, "Energy 70," Las Vegas, Nev., Sept. 21-25. 1970. Vol. 1, pp. 13-19. 
4. R. F. Gribbie and F. L. Kibe, "Low-Compression Theta Pinch with Separated Shock Heating," Los Alamos Scientific Laboratory Report LA-4194-MS (June 1969).

5. F. L. Ribe in "Controlled Thermonuclear Research at LASL: Present Status and Future Pians for Feasibility and Reactor Experiments" Los Alamos Scientific Laboratory Report LA-4656-MS (June 1971), p. 23.

6. F. L. Ribe, "Parameter Study of a Long, Separated-Shock 0 Pinch with Superconducting lnductive Energy Storage," Los Alamos Scientific Laboratory Report LA-4828-MS (December $1971 !$.
7. S. C. Burnett, W. R. Ellis, T. A. Oliphant, and F. L. Ribe, "A Reference Theta Pinch Reactor (RTPR): A Study of a Puised High-Beta Fusion Reactor Based on the Theta Pinch," Los Alamos Scientific Laboratory Report LA-5121-MS (December 1972).

8. F. L. Ribe, S. C. Burnett, W. R. Ellis, "Pulsed High-Beta Fusion Reactor:" U.S. Patent 3,748,226, July 24, 1973. 


\section{BASIC CONCEPTS OF THE STAGED THETA-PINCH SYSTEM*}

Based on the magnitude of plasma confinement times and the method used to heat the plasma, three schemes to obtain magnetically confined thermonuclear fusion can be identified. Methods of confining the plasma by magnetic mirrors operate continuously and require high-energy particle injection. Tokamak and Stellarator confinement schemes operate in a quasi-steady-state mode (10 to 1000 sec), and combines ohmic/injection heating. The proposed theta-pinch reactor will operate in a purely pulsed fashion; heating of the plasma is achieved by a combination of nonisentropic, implosive compression and near isentropic compression. The thermonuclear burn will occur for $\sim 0.1$ sec once every cycle.

Unlike other CTR concepts, the theta-pinch reactor being considered is a high-beta device $(\beta \equiv$ (plasma kinetic pressure)/(magnetic pressure) $\sim 1$ ); very little penetration of either the implosion or compression field into the fully ionized plasma occurs. Figure 2.1-1 illustrates schematically the essential elements of the staged theta-pinch concept. A lowenergy magnetic field, $\mathbf{B}_{\mathbf{S}}$, implodes a fully ionized, low-temperature plasma. The implosion field, which must have a risetime of a few tenths of a microsecond to be effective in heating the plasma, is followed by a slowly rising ( $\sim$ tens of milliseconds) compression ficld, $\mathrm{B}_{0}$, after the radial kinetic energy of the imploding ions is thermalized (equipartitioned). The energy contained by the compression field is significant and must be efficiently recovered. The shaded regions of Fig. 2.1-1 represent a magnetic field perpendicular to the plane of the figure. The arrows indicate the flow of energy into and out of the sysicm. As the plasma is reduced in radius, the density and temperature increase isentropically. Shortly before attainment of the maximum compression field, the plasma ignites and the thermonuclear burning accelerates at a constant compression field, $B_{0}$. The energetic alpha particles (a D- $T$ cycle is being considered) thermalize in the dense plasma and the internal energy of the plasma increases proportionately. The plasma, therefore, expands against the constant field, $\mathbf{B}_{f}$, doing useful (direct conversion) work in the process. At some point in time the compression field is reduced to a value $\mathbf{B}_{\mathbf{Q}}$, the density and temperature decrease, doing more work on the magnetic field, and the thermonuclear

*R. A. Krakowski, T. Oliphant, F. L. Ribe, LASL. 


\subsection{IMPLOSION HEATING}

Figure 2.1-2 illustrates the kind of magnetic-piston or implosion heating being considered. The impiosionheating coil shown is a theta-pinch coil (shown in Fig. 2.1.2 with tivo voltage feed slots) carrying azimuthal current derived from a high-voltage, low-impedance source. Ideally, the magnetic field advances radially inward on a field-free plasma, projecting the ions forward at rwice the piston velocity from a thin plasma sheath. The Alfvén and acoustic Mach numbers are very high, and, strictly speaking, no shock front exists ahead of the piston; the term "implosion heating" is preferred.

To illustrate the physical aspects of the implosion heating process, a summarized derivation of the scaling equations for the "simple" implosion is presented. The detailed analysis of the implosion process with numerical results is given in Appendix $A$. The results for the "simple" implosion are modificd de facto to include equilibration after implosion heating as well as free expansion. Based on the conserv tion of energy and momentum, and assuming that no equilibration occurs in the implosion-heated plasma prior to application of the adiabatic compression field, the following expression gives the sheath velocity during implosion. (The units used in this and following equations are expressed in $\mathrm{cm}, \mathrm{sec}, \mathrm{keV}$, $k \mathrm{G}$, volts, and mTorr.)
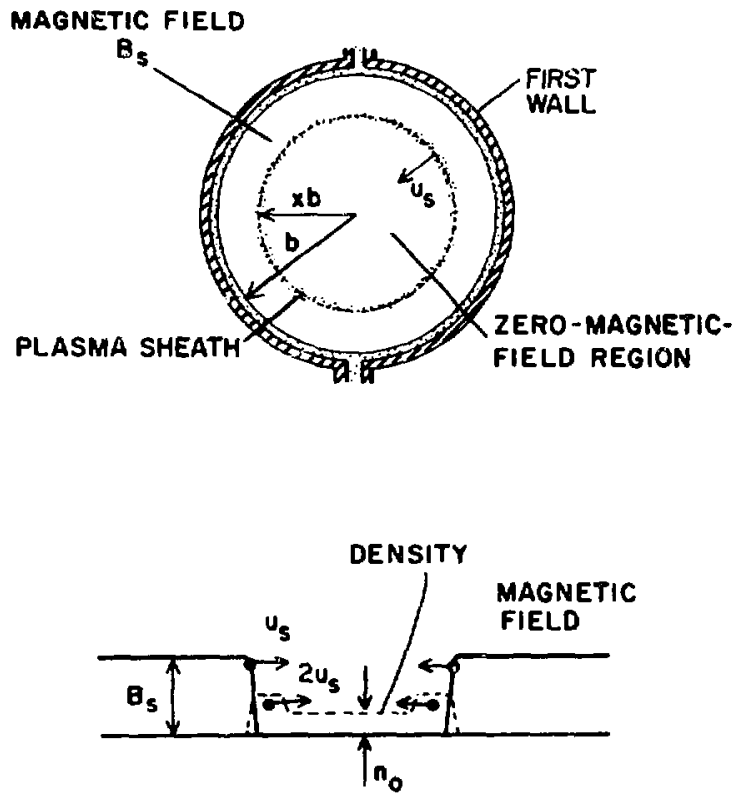

Fig. 2.1-2.

Processes involved in an ideal implosion beating of tbe preionized plasma. $\mathrm{u}_{\mathrm{s}}=2.43 \times 10^{7}\left[\mathrm{kT}_{\mathrm{SH}}\right]^{1 / 2} \cdot(2.1-1)$

By using momentum and pressure balances in conjunction with Eg. (2.1-1), the compression ratio for the quiescent plasma, $\mathrm{x}_{\mathrm{SH}}$, can be shown to equal $\mathbf{0 . 6 3 2}$.

During implosion, the moving plasma surface induces an azimuthal back emf, $V_{s}(\mathrm{keV})$, around the inside surface (defined by radius $b$ ) of the discharge tube equal to $2 \pi \times 10^{-6} \quad b u_{s} B_{s}$. The resulting azimuthal electric field, $\mathrm{E}_{\theta}=\mathrm{V}_{\mathrm{s}} / 2 \pi \mathrm{b}$, is given by

$$
\mathrm{E}_{\theta}=0.65 \mathrm{P}_{\mathrm{A}}^{1 / 2}\left[\mathrm{kT}_{\mathrm{SH}}\right] \text {. }
$$

where $\mathbf{P}_{A}$ represents the $D-T$ filling pressure in $m$ Torr at $297 \mathrm{~K}$. Substitution of Eq. (2.1-1) into the sheath momentum balance yields a relationship between the shock field, $B_{S}(k G)$, the filling pressure, and the quiescent shock temperature, $\mathrm{kT}_{\mathbf{S H}_{\mathrm{H}}}(\mathrm{keV})$,

$$
\mathrm{B}_{\mathrm{S}}^{2}=7.14 \mathrm{P}_{\mathrm{A}}\left[\mathrm{kT}_{\mathrm{SH}}\right] \text {. }
$$

If the ion-electron collision time is large compared to the time when adiabatic compression is induced, the ion temperature after compression $T_{0}$, is related to $T_{S H}$ by

$\mathrm{T}_{0}=\mathrm{T}_{\mathrm{SH}}\left(\mathrm{x}_{\mathrm{SH}} / \mathrm{x}_{0}\right)^{2(\gamma-1)}$.

where $x$ is the radial compression ratio, $\gamma=(2+f) / f$, and $f$ represents the number of degrecs of freedom associated with the ions. The required pressure balance among ions, $n k T=B^{2} / 8 \pi$, leads to the following relationship between $B_{0}(k G)$ and $k T_{0}(k e V)$.

$\mathrm{B}_{0}^{2}=2.86 \mathrm{P}_{\mathrm{A}}\left[\mathrm{kT} \mathrm{T}_{0}\right] / \mathrm{x}_{0}^{2}$.

Equations (2.1-1) through (2.1-5) represent the fundamental design relationships for the simple, implosionheated theta pinch. When implosion hearing is accomplished by a particular programmed magnetic field (free expansion)* ${ }^{*} x_{\text {SH }}$ equals 0.76 (Ref. 7). Furthermore, the

-The particular case of free expansion implosion heating is achieved by applying an initial field equal to $B_{S^{\prime}} / \sqrt{2}$ for a time to achieve a compression ratio of $1 / 3$, removal of the field, and the application of a larger field equal to $\mathbf{B}_{\mathbf{S}}$ after a period of zero field equal to $50 \%$ of the time the initial field is applied. When the field $B_{S} / \sqrt{2}$ is removed, all ions initially projected from the sheath will have passed through the axis of the discharge and impinged again upon the sheath. Upon application of the larger field, $B_{\mathbf{S}}$, all ions have advanied to the opposite side and been reflected with velocity $2 u_{S}$. 
assumptions that equilibration occurs before compression and the effectiv: mass number is 2.5 (instead of 2 used in the foregoing d(velopment), lead to the following set of equations,

$$
\begin{aligned}
& \mathrm{u}_{\mathrm{B}}=1.29 \times 10^{7}\left[\mathrm{kT}_{\mathrm{SH}}\right]^{1 / 2} \\
& \mathrm{E}_{\sigma}=0.202 \mathrm{P}_{\mathrm{A}}^{1 / 2}\left[\mathrm{kT}_{\mathrm{SH}}\right] \\
& \mathrm{B}_{S}^{2}=4.93 \mathrm{P}_{\mathrm{A}}\left[\mathrm{kT}_{\mathrm{SH}}\right] \\
& \mathrm{B}_{0}^{2}=5.71 \mathrm{P}_{\mathrm{A}}\left[\mathrm{kT}_{\mathrm{O}}\right] / \mathrm{x}_{\mathrm{O}}^{2} .
\end{aligned}
$$

The units used in these equations are those used in Eq. (2.1-1). This set of equations illustrates the advantages of a programmed implosion and are used hereafter as scaling equations. By using the isentropic relationship, $\left(B_{O} / B_{S}\right)=\left(x_{S H} / x_{O}\right) \gamma$, one can elininate $P_{A}$ and $k T_{S H}$ from these scaling relationships to yield

$$
\begin{aligned}
& E_{\theta}=.244\left(\mathrm{x}_{0}\right)^{7 / 3}\left[\mathrm{kT}_{0}\right]^{1 / 2} \mathrm{~B}_{0}(2.1-10) \\
& \mathrm{B}_{S}=4.314\left\{\left[\mathrm{~B}_{0}\right]^{2 / 5} \mathrm{E}_{\forall} /\left[\mathrm{kT}_{0}\right]^{1 / 2}\right\}^{5 / 7}
\end{aligned}
$$

Equations (2.1-10) and (2.1-11) are used in determining $\mathrm{E}_{\theta}$ for first-wall design purposes as well as $\mathrm{B}_{\mathbf{S}}$, the magnitude of the implosion heating field. Generally, from selected values of $x_{0}$ and $k T_{0}$ together with the required final density, $n_{0}=n_{A} / x_{0}^{2}$, the value of $B_{0}$ is determined. $B_{0}$ (or a given $n_{0}$ ) will yield the resulting value of $E_{\theta}$.

\subsection{D-T BURN DYNAMICS}

\subsubsection{The Burn Cycle}

When a plasma begins to burn, the alpha-particle reaction products thermalize within the plasma, thereby increasing the internal energy of the ions and electrons. In a low-beta plasma confined by a constant magnetic field, $B_{0}$, the plasma pressure increases and the plasma beta may increase. The pressure in a unit beta plasma, however, remains constant and works against the magnetic pressure, $\mathrm{B}_{0}^{2} / 8 \pi$. This work represents a direct conversion of thermonuclear energy into electrical energy.
The essential elements of a pulsed theta-pinch reactor cycle are illustrated in Fig. 2.1-1. The implosion heating stage is shown in Fig. 2.1-1A where a magnetic field $B_{S}$ of risetime $\sim 0.10 \mu \mathrm{sec}$ and magnitude $\sim 14 \mathrm{kG}$ implodes the fully ionized plasma. After the ion energy associated with the radially directed motion of the plasma has equilibrated between electrons and jons, the temperature is $T_{E}$ and the compression ratio is $\mathrm{x}_{\mathrm{E}}$. The lightly shaded areas in Fig. 2.1-1 represent a magnetic field perpendicular to the risetime the plane of the figure. The adjabatic compression field is then applied by energizing the compression coil. As illustrated in Fig. 2.1-1C, the risetime of the compression field is $31.4 \mathrm{msec}$ and the maximum value is $\sim 110 \mathrm{kG}$. The precise definition of the rise time, $\tau_{\mathbf{R}}$, is given by Eq. (2.2-1). Energy flows into the compression coil at this point, as indicated by the arrow in Fig. 2.1-1C. As the plasma is compressed and the burn occurs, the 3.5-MeV alpha particles exchange energy with the ions and electrons, and the plasma internal energy is increased; the plasma expands against the constant maratic field and useful work is performed (see Fig. 2.1-1D).

The magnetic compression and decompression cycle, accompanied by important energy quantities, is shown in Fig. 2.2-1. The rising portion of the adiabatic compression field is described by

$$
B_{0}\left(t^{\prime}\right)=B_{s}+\left(B_{0}-B_{s}\right) \sin ^{2}\left(\frac{\pi}{2} \frac{t^{\prime}}{\tau_{R}}\right)
$$

and the falling portion of the compression field is given by

$$
B_{0}\left(t^{\prime \prime}\right)=B_{Q}+\left(B_{0}-B_{Q}\right) \cos ^{2}\left(\frac{\pi}{2} \frac{t^{\prime \prime}}{\tau_{R}}\right) \text {. }
$$

In these expressions, $B_{0}$ is the maximum compression field, $\mathrm{B}_{\mathbf{S}}$ is the implosion heating field, $\mathrm{B}_{\mathrm{Q}}$ is the quench ficld, $t^{\prime}$ is measured from point $a$ in Fig. 2.2-1, and $t^{\prime \prime}$ is measured from point $c$. The adiabatic compression field is held at the maximum value $B_{0}$ for 0.070 sec., and 0.031 sec was used for $\tau_{R} \cdot$ * After the ion-electron equilibration which follows the implosion heating stage, the compression field rises in $31.4 \mathrm{msec}$ to a peak value of $110 \mathrm{kG}$ at

Approximating the waveform described by Eqs. (2.2-1) and (2.2-2) by a linearly rising (and falling) curve with a slope evaluated at the inflection point gives a nominal tise (fall) time of 20 msec. The effective burn time which results from this approximation is 80 msec. Whenever calculations do not require the exact waveform, the nominal values of $\tau_{R}$ and $\tau_{B}$ are used. 


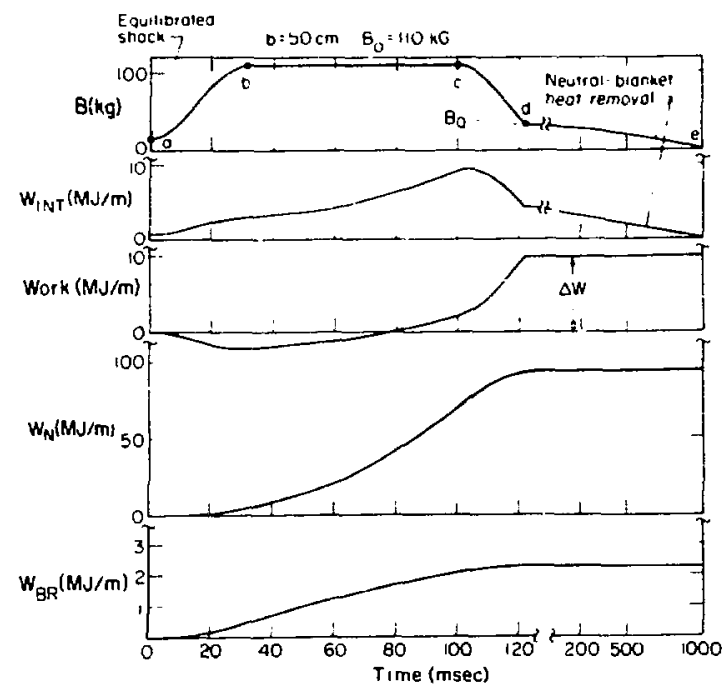

Fig. 2.2-1.

Time bistory of the magnetic field used for adiabatic compression and time bistory of important energy parame ters

$W_{I N T}$ : Total internal energy of plasma including the contribution from alpha particles;

$\Delta W$ : Direct conversion work done by the plasma expanding against the compression field;

$W_{N}$ : Total thermonuclear energy deposited in the blanket by s.eutrons;

$W_{B R}$ : Total bremsstrablung energy radiated from plasma.

point $b$, as energy is transferred to the compression coil from a cryogenic magnetic energy storage. ${ }^{7}$ The major portion of the D-T burn occurs between points $b$ and $c$ under conditions of constant magnetic pressure. $B_{0}^{2} / 8 \pi$. The compression field is reduced at point $c$ and attains a value of $B_{0}=32 \mathrm{kG}$ as point $d$, most of the magnetic energy being permitted to flow back into the magnetic energy storage.

At the end of the quench stage, point $d$, the D-T reaction rate is negligibly low ( $\mathrm{kT} \sim 9 \mathrm{keV})$. On a much longer time scale, the magnetic field is programmed to decrease slowly, thereby preventing the hot plasma from touching the first wall. During the period from points $d$ to e, the plasma is maintained at a constant radius and internal energy is transferred through a neutral gas blanket to the reactor blanket. 8,9

The alpha-particle thermalization, plasma heating, and plasma expansion are compured numerically by a Fokker-Planck calculation, ${ }^{10}$ which accounts for the timedependent inagnetic field. These calculations indicate that ignition occurs slightly prior to point $b$, and the burn continues almost to point $d$. Shown as a function of time on Fig. 2.2-1 are the plasma internal energy, $W_{1 \mathrm{NT}}$, the energy deposited in the blanket by neutrons $\left(Q_{N}=20.5 \mathrm{MeV} /\right.$ neutron), $W_{N}$, and the bremsstrahlung energy, $W_{B R}$. Figure 2.2-2 summarizes the timedependence of the plasma radius, ion density, alphaparticle density, plasma temperature, and alpha-particle energy. Numerical values from these computations are used to detei mine the overall energy balance.

The nominal 30-msec risetime of the compression field represents a first estimate of an optimal process. The inertia associated with the massive machinery that generates the compression field dictates large values for $\tau_{R}$, whereas the need to maintain the implosion-heating field for times of the order of milliseconds introduces complexities into the design of the implosion-heating power supply. The values used for $\tau_{R}$ and $\tau_{B}$ (time from point $b$ to point $c$ on Fig. 2.2-1) represent a compromise between the long risetime requirements of the METS system and

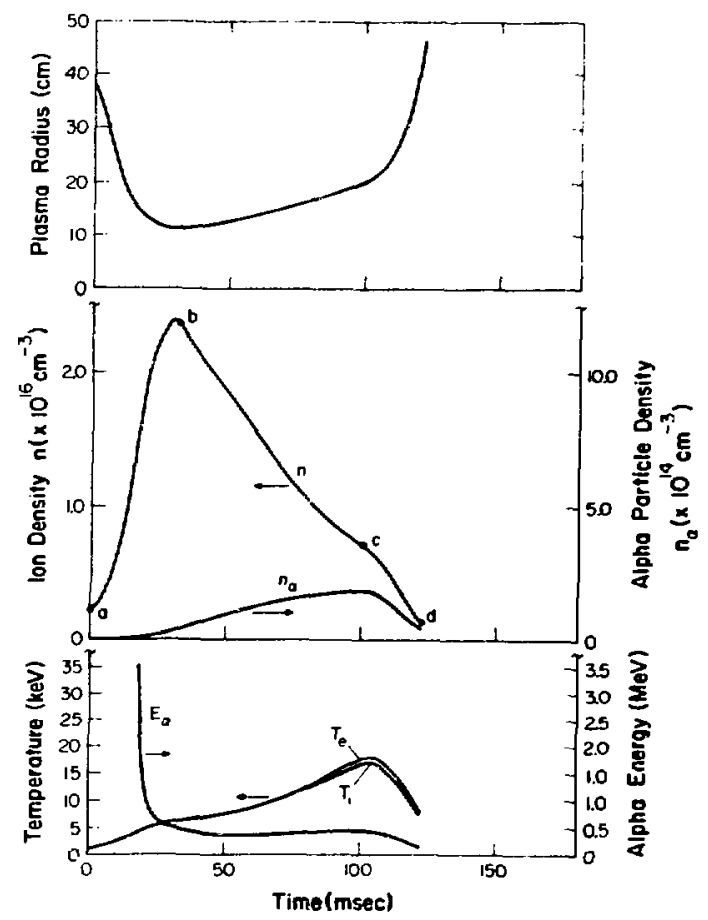

Fig. 2.2-2.

Time bistory of plasma radius, ion density (n), alpha particle density $\left(n_{\alpha}\right)$, alpha particle energy $\left(E_{\alpha}\right)$, electron temperature $\left(T_{e}\right)$, and ion temperature $\left(T_{i}\right)$. 
the benefits accrued in the implosion-heating system by use of short risetimes. For a given $\tau_{R}$ the value of $\tau_{B}$ is selected to ensure an abundance of thermonuclear energy. Figure 2.2-3 indicates the dependence of the neutron energies deposired into the blanket, $W_{N}$, on $\tau_{B}$ for various values of $\tau_{R}$. The dependence of the direct conversion efficiency, $\eta_{D C}=\Delta W / W_{\alpha}$ (discussed in Sic. 2.2.2) on $\tau_{R}$ and $\tau_{\mathrm{B}}$ is also displayed, as are the present design points. As discussed in Sec. 4.3, design restraints on the METS system indicate that $\tau_{\mathrm{R}}$ may have to be increased, and the data presented in Fig. 2.2-3 clearly dernonstrate that problems with ignition, energy release, and direct conversion are not expected if increases in $\tau_{R_{R}}$ are dictated by design requirements imposed by other parts of the RTPR system.

\subsubsection{The Direct Conversion Cycle}

The burn cycle is represented in the form of a pressure-volume diagram in Fig. 2.2-4 and the various stages of the cycle defined by Fig. 2.2-1 are injicated. Figure 2.2-5 interprets the burn cycle in terms of a temperature-entropy diagram. In both cases the portion of the cycle between points $e$ and a in Figs. 2.24, and 2.2-5 is designated by a dashed line to indicate uncertainty in the path to be taken from the end of the cooling stage through the end of the implosion-heating stage. The direct conversion work, $\Delta W$, generated throughout each cycle is represented by the area encompassed by the curve on Figs. 2.2-4 and 2.2-5.

The total energy released in the form of alpha-particle energy is $15.8 \mathrm{MJ} / \mathrm{m}$ and $\Delta W=9.8 \mathrm{MJ} / \mathrm{m}$; the intrinsic

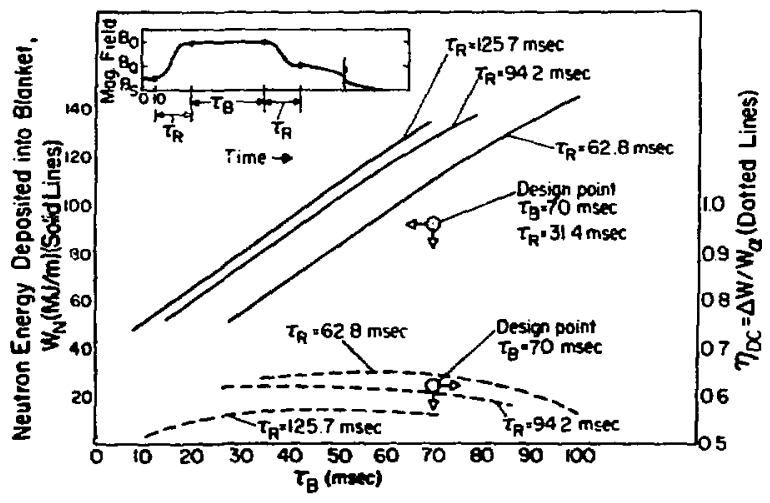

Fig. 2.2-3.

Dependence of neutron energy deposited into blanket, $W_{N}$, and direct conversion efficiency, $\eta_{D C}=\Delta W / W_{\alpha}$, on rise time of the compression field.

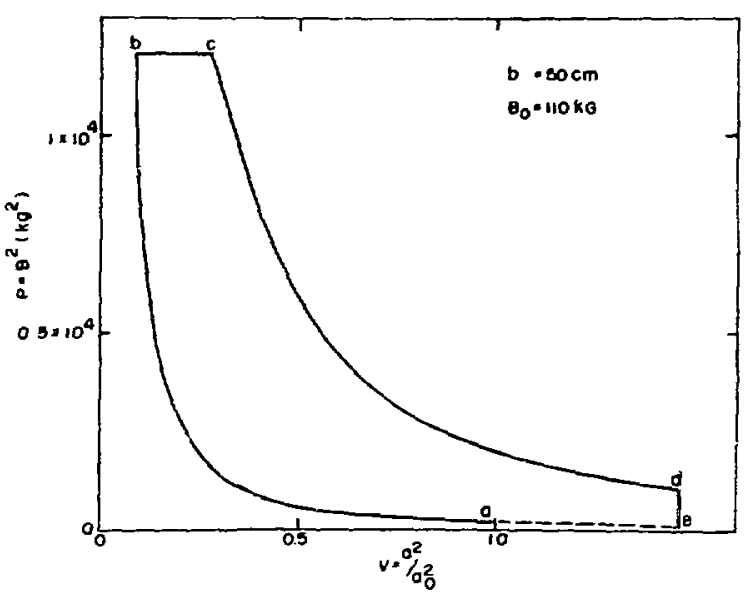

Fig. 2.2-4.

Depiction of thermonuclear burn cycle on a pressure-volume diagram.

a: plasma radius;

$a_{o}$; initial plasma radius;

B: compression field.

thermodynamic efficiency of the direct conversion cycle is $62 \%$, and $\Delta \mathrm{W}$ is available to the reactor system at $\sim 100 \%$ efficiency. The thermonuclear energy, $w_{N}=93.1 \mathrm{MJ} / \mathrm{m}$, however, is available only for thermal conversion with an efficiency $\eta_{\mathrm{TH}}$. The ratio of

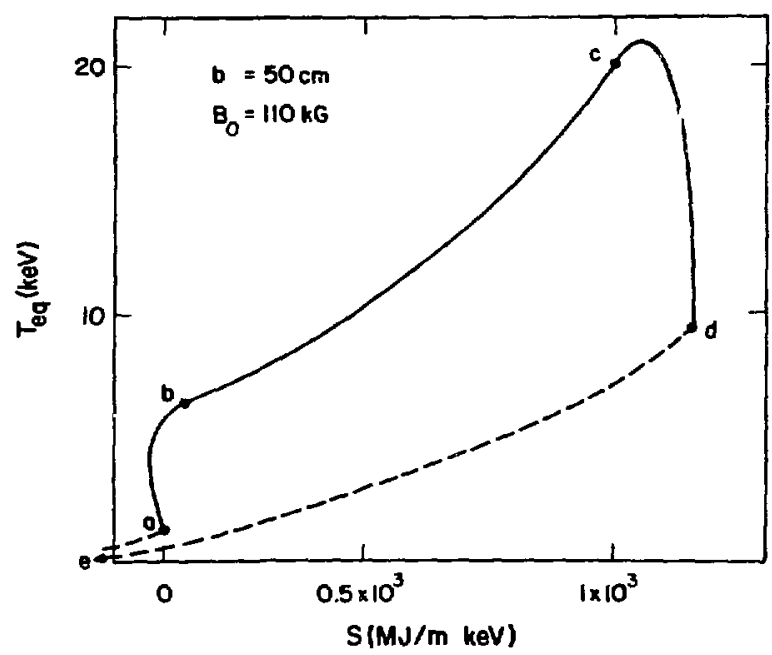

Fig. 2.2-5.

Depiction of thermonuclear burn cycle on a temper. atureentropy diagram. 
direct-conversion energy to thermally converted energy is

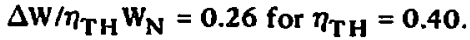

The direct conversion work has an appreciable effect on the net plant efficiency, $\eta_{p}$, and the circulating power frartion, $\epsilon$, for the theta-pinch reactor; in a sense the direct conversion process represents a to ping cycle for the thermal conversion plant.

\subsection{PLASMA COOLING BY NEUTRAL GAS LAYER}

An attractive feature of a pulsed fusion reactor is the possibility of removing the alpha-particle "ash" resulting from the burnup of the deuterium-tritium fuel mixture and injecting fresł fuel between burning pulses. No divertor is required, as in the case of a steadystate, toroidal reactor. A iayer of neutral gas injected between the hot central plasma and the first wall is used to cool, neutrahize, and purge the partially burned D-T plasma. Preiiminary calculations show that sputtering problems are alleviated because heat transfer to the wall, which would otherwise occur by energetic ions, will now occur primarily by means of low-energy neutral atoms and to a lesser extent by ultraviolet and visible radiation.

The fuel cyciing is accomplished in the following manner. It the end of the burning phase (point $c$ in Fig. 2.2-1), the reaction is quenched by suddesly reducing the confining field to a value which allows the hot central plasma to expand to a radius which is close to that of the wall. Cold, neurral gas is rapidly introduced into the space between the central plasma and the wall at some point during the thermonuclear bum. The central plasma is cooled by the outward radial flow of heat through this gas layer to the wall. The confining field, which is programmed to maintain the outer radius of the plasma at a fixed value, decreases with time as the plasma is cooled. After the central plasma has lost most of its energy and is neutralized, it is removed by vacuum pumps.

In calculating the rate of heat transfer throughout the cooling stage, ${ }^{8}$ it is assumed that the cooling layer is furmed instantaneously after quenching by the introduction of a neutral gas of uniform density, $n_{B}$, and remperature, $T_{w}$. Introduction of the cooling gas at times before the quench is also being considered. A quasisteady tomperature profile is quickly established by heat conduction through the gas layer to the wall. The thermal conduction within the plasma is high enough to maintain an essentially uniform temperature profile through the plasma region, since the plasma contains litcle or no magnetic field $(\beta \sim 1)$. Therefore, the central plasma is represented in the calculation by a rigid, isothermal cylinder with a temperature $T_{a}$, which decreases in time in accordance with the amount of energy extracted. Although the plasma radius, $x_{Q} b$, and the wall radius, $b$, are fixed, internal motion occurs as a result of pressure gradients arising from the changing temperature profile as heat flows. The degree of ionization at various points will also vary with changing densities and temperatures. The assumption is made that, at each point in the gas layer, the plasma and neutral gas are in thermal and ionization equilibrium. The plasma and neutral components are assumed to move together as a single fluid. Although the pressure gradients of the separate components will tend to drive a relative motion, " proper inclusion of the $\vec{v} \cdot \nabla \vec{v}$ terms in the momentum equations. $^{8}$ Another assumption is that the D-T blanket gas enters in the atomic rather than in the nolecular state. This situation is not totally real, since the gas is assumed to enter at the wall temperature. Although molecular dissociation can be inclucled in the calculation, it is not expected to be significant; the dissociation energy is sinall compared to atomic ionization and excitation energies, and the change in thermal conductivities between atomic and molecular gases is not significant compared to that between ionized and rieutral gases in the presence of a magnetic field.

The initial conditions for the cooling calculation are the conditions obtained from the burn calculation at the time corresponding to point $\mathrm{d}$ in Fig. 2.2-1. The total internal energy of the plasma at that time is $4.13 \mathrm{MJ} / \mathrm{m}$. Since energy equipartition among the alpha particles, ions, and electrons takes place in a time short compared to the cooling time, equilibration is assumed to take place instantaneously as cooling begins. For the final burn conditions the equilibrated plasma temperature is $\mathbf{9 . 6 2}$ $\mathrm{keV}$. A family of computed curves for the central plasma temperature $T_{2}$ vs time for different values of average cooling layer diensity, $n_{B}$ has been plotred in Fig. 2.3-1. The intercepts of these curves with the time axis give the cooling time as a function of the density $n_{B}$, and this function is plotted in Fig. 2.3-2. As seen from this figure, a density $n_{B}$ of $1 \times 10^{14} \mathrm{~cm}^{-3}$ gives the design cooling time of: sec.

Temperature profiles at various times for $\mathrm{n}_{\mathrm{B}}=1 \times 10^{14}$ c ${ }^{3}$ are plotted in Fig. 2.3-3. The "break point" exhibiteu by these profiles occurs at the arnsition from ion to neutral atom heat conduction and reflects the fact that atom thermal conduction is much greater than ion thermal conduction; atom thermal conduction, therefore, requires a temperature gradient which is orders of magnitude smaller than ion thermal conduction.

An important feature which emerges from examination of Fig. 2.3-1 is that the centraj plasma temperature drops approximately linearly with time. To a good approximation, it is possible simply to use a constant value for heat flow through the first wall in estimates of therrial loadings during the plasma cooling stage. The physical 


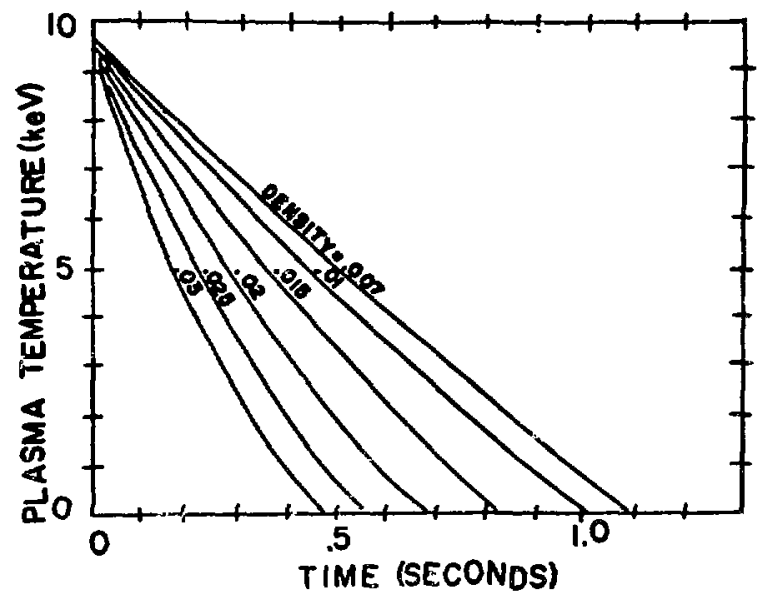

Fig. 2.3-1.

Plasma ten.perature as a function of time and density $\left(10^{16} / \mathrm{cm}^{3}\right)$ of neutral gas density.

reason for the almost constant rate of heat flow is that the thermal conductivity in the region of maximum slope in the curves shown in Fig. 2.3-3 remains almost constant in time; this region of the curve primarily governs the heat transport.

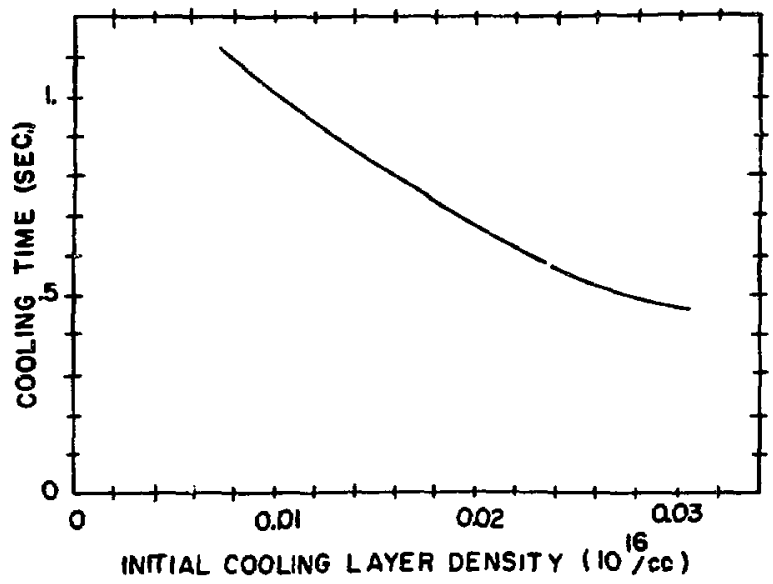

Fig. 2.3-2.

Time required to cool the plasma vs density of the initial cooling iayer $\left(10^{16} / \mathrm{cm}^{3}\right)$.

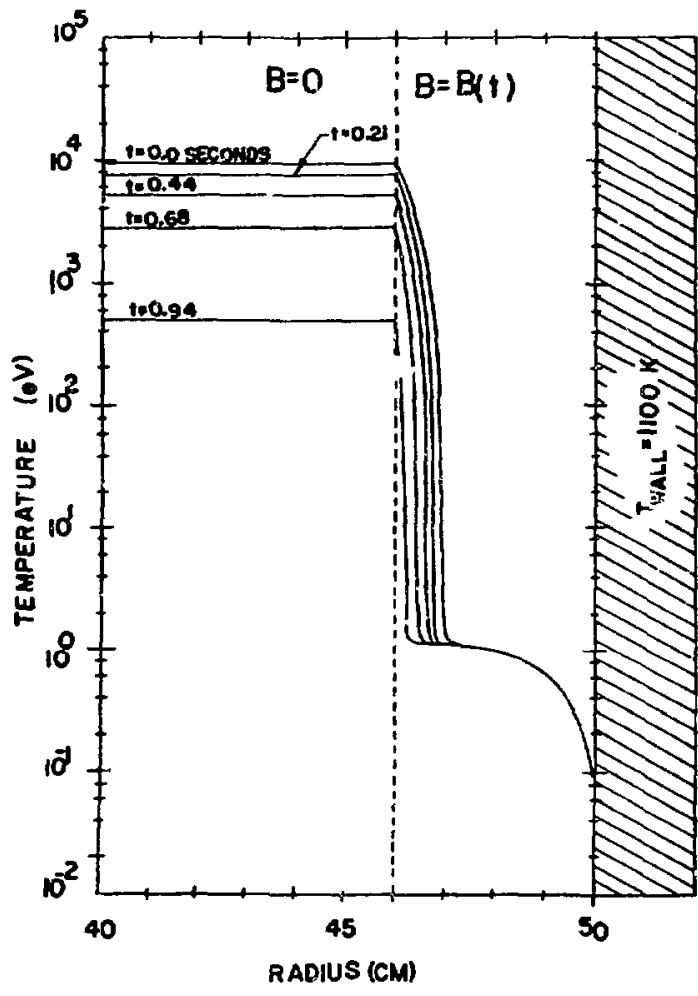

Fig. 2 3-3.

Typical temperature profiles in cooling gas layer as a function of time.

\subsection{ENERGY BALANCE AND CONVERSION}

The energy from the $\mathrm{D}-\mathrm{T}$ thermonuclear reaction appears in the form of energetic alpha particles $(3.52$ $\mathrm{MeV}$ ) and neutrons (14.1 MeV). About $62 \%$ of the energy associated with the alpha particles is channeled into the magnetic store by direct conversion (Sec. 2.2). The remainder of the alpha-particle energy is either transferred to the electrons, which radiate bremsstrahlung to the first wall, or is extracted by heat flow through the cooling layer as discussed in Sec. 2.3. Most of the neutron energy is deposited in the blanket. Neutron multiplication in the blanket and exoergic nuciear transmutation reactions generate additional energy. The details of the blanket neutronics are discussed in Sec. 4.5. The bremsstrabiling energy deposited in the first wall during the burn, although not a significant fraction of the net energy produced, must be taken into account insofar as avoidance of excessive thermal transients at the first wall is concerned. 
All thermal energy generated in the blanket by neutron, gamma ray, bremsstrahlung, and plasma cooling is eventually transferred to the lithium coolant. This energy is used by a thermal conversion system to generate electrica! energy. Two thermal conversion systems are considered. One is a conservative and conventional steam plant operating with a maximum steam temperature of $755 \mathrm{~K}$ and a correspondingly low thermal efficiency $\left(\eta_{\mathrm{TH}}=0.40\right)$. The second power conversion system is an advanced steam plant which uses a potassium topping cycle. The maximum potassium temperature is $1273 \mathrm{~K}$ and results in a thermal efficiency of $\eta_{\mathrm{TH}}=0.56$. Both cycles are discussed in Chapter 3 along with details of the heat exchanger, turbines, piping, and the rationalization for the plant choice. Both plants operate with a $127 \mathrm{~K}$ temperature rise in the lithium coolant; the conventional plant uses lithium at an inlet temperature of $820 \mathrm{~K}$, whereas $1273 \mathrm{~K}$ is used for the advanced power plant. The overall energy balance for the RTPR is presented for both the conventional and advanced power conversion systems.

Important plasma parameters at the start of and during the burn are summarized in Tables 2.4-1 and 2.4-2. The overall energy balance for the RTPR based on these design parameters is depicted schematically in Fig. 2.4-1. The total energy content of the plasma plus the magnetic energy contained within the reactor core is shown as a function of time throughout the power cycle (impiosion heating $\rightarrow$ adiabatic compression $\rightarrow$ burn $\rightarrow$ quench $\rightarrow$ cooling). In Fig. 2.4-1 time flows horizontally from left to right. Major sources of energy input into the reactor core are indicated temporally in the lower portion of Fig. 2.4-1 and consist of the implosion heating energy, $W_{\mathrm{SH}}$, the energy circulating in the METS, $W_{B}$, the transport curren، losses in the compression coil, $W_{T}(C u)$, eddy current losses in the reactor core, $W_{E}(\mathrm{Li}), W_{E}(C), W_{E}(\mathrm{Cu})$, and auxiliary energy needed throughout the power cycle, $w_{A}$.

The fraction 0.623 of the total energy in the METS, $W_{B O}$. is cycled between the cryogenic storage system and the reactor ccre. The energy $W_{B}$ flows into tile reactor core during the adiabatic compression and the amount $\left(1-n_{\mathrm{B}}\right) \mathrm{W}_{\mathrm{BO}}$ is assumed irrecoverably lost during the power cycle.* The fraction $\eta_{\mathrm{DC}}$ of the total alpha-particle energy, $w_{\alpha}$, is recovered as direct conversion energy and is returned directly to the METS. The total power circulated during the power cycle, $w_{C}$, obtained from the balance indicated schematically on Fig. $2.4-1$, is

\footnotetext{
" $\eta_{B}$ describes the total METS efficiency with the exclusion of transport and eddy current losses in the compression coil; joule losses in the compression coil are taken into account separately.
}

TABLE 2.4-1

\section{PLASMA PARAMETERS AT START OF BURN}

\begin{tabular}{|c|c|c|}
\hline Symbol & Definition & Value \\
\hline $\mathbf{B}_{\mathbf{0}}$ & Compression field & $110 \mathrm{kG}$ \\
\hline$x_{0}$ & $\begin{array}{l}\text { Compression ratio at start of } \\
\text { burn }\left(a_{0} / b\right)\end{array}$ & 0.226 \\
\hline $\mathbf{b}$ & Radius of first wall & $50 \mathrm{~cm}$ \\
\hline $\mathrm{kT}_{0}$ & $\begin{array}{l}\text { Electron and ic } n \text { temperature at } \\
\text { start of burn }\end{array}$ & $\sim 6.1 \mathrm{keV}$ \\
\hline$\stackrel{a}{o}_{\mathbf{k T}_{\text {SH }}}$ & $\begin{array}{l}\text { Plasma radius at start of burn } \\
\text { Temperature of ions after implo- } \\
\text { sion heating (before } \\
\text { equilibration) }\end{array}$ & $\begin{array}{r}\sim 11.3 \mathrm{~cm} \\
2.28 \mathrm{keV}\end{array}$ \\
\hline $\mathrm{kT}_{\mathrm{E}}$ & $\begin{array}{l}\text { Electron and ion semperature after } \\
\text { implosion heating (after } \\
\text { equilibration) }\end{array}$ & $1.14 \mathrm{keV}$ \\
\hline $\mathbf{B}_{\mathbf{S}}$ & Implosion heating field & $13.8 \mathrm{kG}$ \\
\hline$u_{s}$ & Implosion velocity & $20.1 \mathrm{~cm} / u \mathrm{sec}$ \\
\hline & Plasma back emf & $650 \mathrm{kV}$ \\
\hline $\mathrm{E}_{0}$ & Implosion heating electric field & $2.0 \mathrm{kV} / \mathrm{cm}$ \\
\hline $\mathbf{P}_{\mathbf{A}}$ & Ambient filling pressure ( $300 \mathrm{~K}$ ) & 17 mTorr \\
\hline $\mathbf{n}_{\mathbf{0}}$ & $\begin{array}{l}\text { Ion density }\left(n_{D}=n_{T}=\frac{n_{0}}{2}\right) \text { at } \\
\text { start of burn }\end{array}$ & $2.46 \times 10^{16} \mathrm{~cm}^{-2}$ \\
\hline$\beta$ & $\begin{array}{l}\text { Ratio of plasma to magnetic } \\
\text { pressure }\end{array}$ & $\sim 1$ \\
\hline $\begin{array}{r}\mathrm{w}_{\mathbf{C}}=\mathrm{Y} \\
+\end{array}$ & $\begin{array}{l}W_{E}(L I)+W_{E}(C)+W_{E}(C u)+ \\
W_{S B}+W_{A}+\left(1-n_{B}\right) w_{B O}-\end{array}$ & $\begin{array}{l}\mathrm{W}_{\mathrm{T}}(\mathrm{Cu}) \\
{ }_{\mathrm{DC}} \mathrm{w}_{\alpha} \cdot \\
(2.4-1)\end{array}$ \\
\hline
\end{tabular}

Included in $W_{C}$ is the make-up energy required per cycle by the implosion heating system, $\mathbf{W}_{\mathrm{SH}}$ This energy is distributed between the irreversible implosion heating losses, $W_{S L}$, and the plasma internal energy at the end of the implosion heating stage, $W_{P}(S)$. The energy $W_{S H}$ is dissipated in each power cycle whether or not a plasma exists, i.e., if the plasma density is zero, then $W_{S H}=W_{S L}$. In general, $W_{S H}$ is equal to the sum of $W_{S L}$ and $W_{P}(S)$.

The energy released from the reactor core at various stages of the puwer cycle is indicated chronologically in the upper region of Fig. 2.4-1. The high-grade (recoverable) thermal energy, $W_{T H}$, is given by

\footnotetext{
-The implosion heating coil can be designed to keep the joule losses in the coil near zeto, and, therefore, this loss is not incorporated into $W_{\mathrm{SH}}$.
} 
TABLE 2.42

PLASMA PARAMETERS DURING, BURN

Symbol

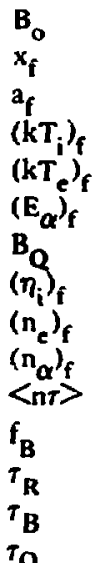

Definition

Adiabatic compression field

Compression iatio at end of burn"

Final plasma radius"

Final ion temperature ${ }^{\bullet}$

Final electron temperature

Final $\alpha$-particle mean energy

Guench field

Final ion density ${ }^{*}$

Final electron density

Final $\alpha$-particle density ${ }^{*}$

Integrated Lawson parameter $\neq$

Fractional fuel burnup ${ }^{\neq}$

Effective compression field rise and fall time

Effective burn time

Time to cool plasma (2 sec used for energy balance)

\begin{tabular}{l} 
Value \\
\hline $110 \mathrm{kG}$ \\
0.40 \\
$20.0 \mathrm{~cm}$ \\
$15.4 \mathrm{keV}$ \\
$17.1 \mathrm{keV}$ \\
$0.45 \mathrm{MeV}$ \\
$32.2 \mathrm{kG}$ \\
$7.19 \times 10^{15} \mathrm{~cm}^{-3}$ \\
$7.55 \times 10^{15} \mathrm{~cm}^{-3}$ \\
$1.80 \times 10^{14} \mathrm{~cm}^{-3}$ \\
$8 \times 10^{14} \mathrm{~cm}^{-3} \mathrm{sec}$ \\
$4.8 \%$ \\
$0.020 \mathrm{sec}$ \\
$0.08 \mathrm{sec}$ \\
$1-3 \mathrm{sec}$
\end{tabular}

- At teginning of quench stage.

At end of quench stage.

$$
\begin{aligned}
& w_{T H}=w_{S L}+w_{E}(L i)+W_{E}(C)+w_{N} \\
& +W_{B R}+W_{I N T}
\end{aligned}
$$

where $w_{N}$ represents the total neutron energy (inelastic + elastic scatterings, transmutations) and $W_{B R}$ is the bremsstrahlung radiation. The energy multiplication factor, $Q$, is defined as the ratio $W_{T H} W_{C}$ and equals $1: \eta_{\mathrm{TH}} \epsilon$, where $\eta_{T H}$ represents the thermal conversion efficiency (assumed to be 0.40 for the conventional power conversion system and 0.56 for the advanced power conversion system) and $\epsilon$ is the circulating power fraction, $\epsilon=w_{C} / \eta_{T H} W_{T H}$. Using the identity $w_{S H}=W_{S L}+W_{P}(S)$, the following expression for $Q$ results.
Table 2.4-3 summarizes the definitions of important energy quantities and gives numerical values based on the plasma parameters listed in Tables 2.4-1 and 2.4-2. The total joule losses incurred per power cycle, $W_{L} \equiv W_{E}(\mathrm{Li})$ $+W_{E}(C)+W_{E}(C u)+W_{T}(C u)+W_{S H}-W_{P}(S)$, amount to $8.03 \mathrm{Mj} / \mathrm{m}$. Compared to the direct conversion energy, $\Delta \mathrm{W}=\eta_{\mathrm{DC}} \mathrm{W}_{\alpha}=9.82 \mathrm{MJ} / \mathrm{m}$, the direct conversion work done during the burn by the plasma expanding against the compression fielu more than compensates for $W_{L}$. The minimum acceptable value of $\eta_{B}$ is 0.86 , i.e., that for which $\epsilon=1.0$; this situation corresponds to a loss of $14.0 \%$ of $W_{B O}$ per power cycle in the METS. Table $2.4-4$ summarizes the RTPR performance for the conditions described in Tables 2.4-1, 2.4-2, and 2.4-3. Figure 2.4-2 presents the overall energy baiance schematically according to the format suggested by Persiani. ${ }^{12}$

$$
Q=\frac{w_{S H}+w_{E}(L i)+w_{E}(C)+w_{N}+W_{B R}-w_{P}(S)+w_{I N T}}{w_{S H}+W_{E}(L i)+w_{E}(C)+w_{E}(C u)+w_{T}(C u)+w_{A}+\left(1 \eta_{B}\right) w_{B O}-\eta_{D C} w_{\alpha}}
$$


TABLE 2.4-3

\section{SUMMARY OF ENERGY BALANCE FOR RTPR}

Symbol

$w_{\text {SH }}$

$\mathbf{w}_{\mathrm{B}}$

$\mathrm{w}_{\text {BO }}$

$\mathrm{W}_{\mathrm{E}}(\mathrm{Li})$

$W_{E}(C)$

$w_{E}(\mathrm{Cu})$

$\mathrm{W}_{\mathrm{T}}(\mathrm{Cu})$

$w_{\alpha}$

$\Delta W$

$w_{N}$

$\mathbf{w}_{\mathbf{B R}}$

$\mathbf{W}_{\mathbf{A}}$

$\mathbf{w}_{\text {TH }}$

$w_{p}(S)$

$\mathrm{W}_{\text {INT }}$

$\mathrm{w}_{\mathrm{sC}}$

$w_{\mathrm{C}}$

W $_{\text {ET }}$

$\mathbf{W}_{\mathbf{E}}$
Definition

Value $(\mathrm{MJ} / \mathrm{m})^{*}$

Energy required for implosion heating system $=f\left(B_{S}{ }^{2} / 8 \pi\right) \pi b^{2}(f \sim 2.79)$ Joule losses in coil per se are estimated to be less than $0.1 \mathrm{MJ} / \mathrm{m}$

Circulating energy in the METS/compression coil system

$=\left(3_{0}{ }^{2} / 8 \pi\right) \pi \bar{R}_{c}^{2}$

Peak magnetic energy in cryogenic system $W_{B} / 0.623$

286

Eddy current losses in the Li portion of blanket $\equiv 0.002 .5 \mathrm{~W}_{B}$

Eddy current losses in the $C$ portion of blanket $\equiv 0.0010 \mathrm{~W}_{B}$

0.16

Eddy current losses in the Cu compression coil $\equiv 0.03 \mathrm{w}_{\mathrm{T}}(\mathrm{Cu})$

0.18

Transport current losses in the compression coil during compression, burn, and quench

5.96

$\frac{2 \pi \bar{R}_{c} \eta_{C u} B_{O}{ }^{2}\left(\tau_{B}+2 / 3 \tau_{R}\right)}{(.4 \pi)^{2} \lambda \Delta R_{C}}\left[1+1 / 2\left(B_{Q} / B_{O}\right)^{2} \tau_{Q} /\left(\tau_{B}+2 / 3 \tau_{R}\right)\right]$

Total alpha particle energy created during burn

15.80

Direct conversion energy generated during burn $\left(\eta_{\mathrm{DC}} w_{\alpha}\right)$

9.82

Neutron energy deposited in blanket $\left(Q_{N}=20.53 \mathrm{MeV} / \mathrm{n}\right)$

93.11

Bremsstrahlung energy during burn

Auxiliary power requirements $=0.05 \eta_{\mathrm{TH}} \mathrm{w}_{\mathrm{TH}}$

$2.03(2.86)$

Total usable thermal energy $\equiv W_{S H}+W_{E}(L i)+W_{E}(C)+W_{N}-W_{P}(S)$

$+W_{B R}+w_{I N T}$

Plasma energy at end of shock phase $=3 n_{e} k T_{E}\left(x_{S H} b\right)^{2}$

0.33

Total plasma internal energy at quench

4.13

5.72

$6.29(7.12)$

$$
+w_{T}(C u)+w_{A}+\left(1-\eta_{B}\right) w_{B O} \cdot \eta_{D C} w_{\alpha}
$$

Gross electrical energy $=\eta_{\mathrm{TH}} \mathrm{w}_{\mathrm{TH}}$

Net electrical energy $=(1-\epsilon) \eta_{T H} w_{T H}$

Value given for $\eta_{\mathrm{TH}} \neq 0.40$, if value in parentheses $\eta_{\mathrm{TH}}=0.564$. 


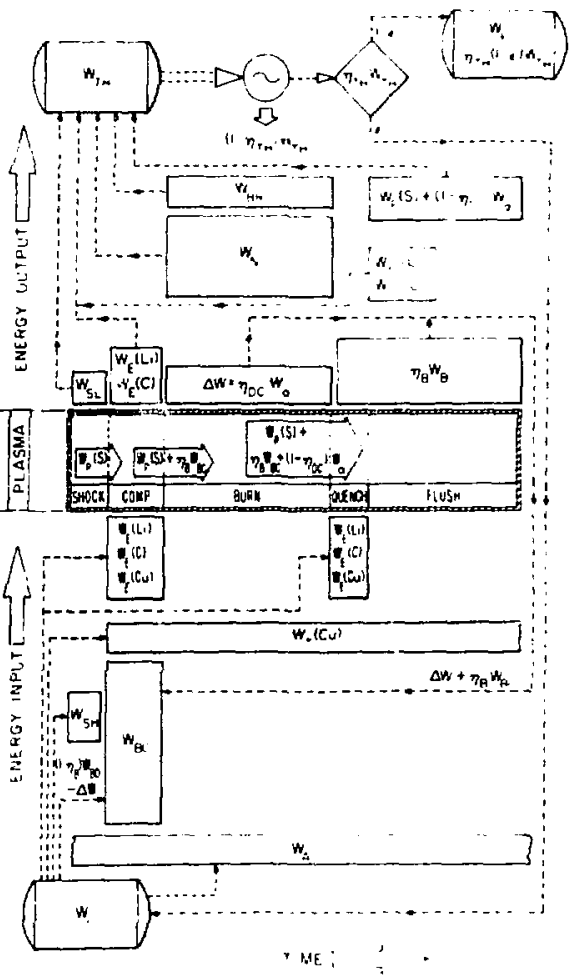

Fig. 2.4-1.

Scbematic diagram of generalized energy balance for a pulsed, theta-pincb fusion reactor. (Refer to Tables 2.5-3 and 2.5-4 for notation.)

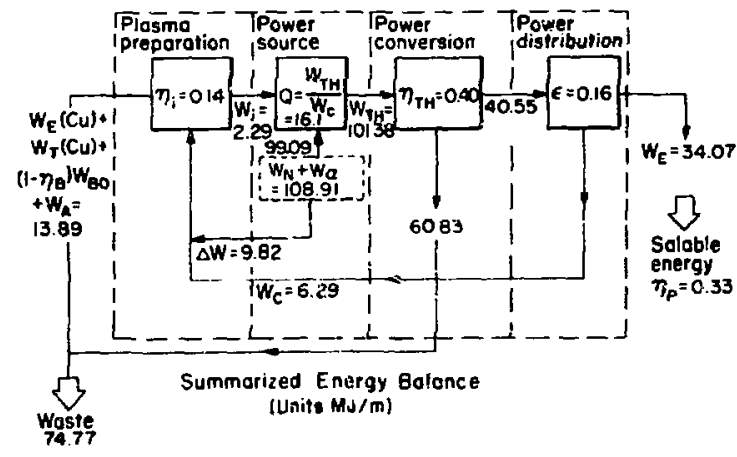

Fig. 2.4-2.

Flow diagram of summarized energy for $R T P R$ which uses a conversional $\left(\eta_{T H}=0.40\right)$ sodiumsteam power conversion system.
TAELE 2.4-4

PERFORMANCE VALUES FOR RTPE

\begin{tabular}{|c|c|c|}
\hline Symbol & Definition & Value \\
\hline $\mathbf{Q}$ & $\begin{array}{l}\text { Energy multiplication factor } \\
=\mathrm{W}_{\mathrm{TH}}{ }^{\prime W_{c}}\end{array}$ & 16.12014 .24 \\
\hline$\epsilon$ & $\begin{array}{l}\text { Recirculating power fraction } \\
=w_{c}{ }^{\prime} \eta_{T H} w_{T H}=1 / \eta_{T H} Q\end{array}$ & $0.16(0.13)$ \\
\hline$\eta_{\mathrm{TH}}$ & Thermal conversion efficiency & $0.40(0.564)$ \\
\hline$\eta_{B}$ & $\begin{array}{l}\text { METS cycle efficiency } \\
\text { (if } \eta_{B}<0.84 \text { then } \epsilon>1.0 \text { ) }\end{array}$ & 0.98 \\
\hline$\eta_{D C}$ & Direct conversion efficiency $=\Delta W / W_{\alpha}$ & 0.62 \\
\hline$\eta_{\mathbf{P}}$ & Nex plant efficiency $=\eta_{\mathrm{TH}}(1 \cdot \epsilon)$ & $0.34(0.45)$ \\
\hline
\end{tabular}

\section{REFERENCES}

1. F. L. Ribe, T. A. Oliphant, Jr., and W. E. Quinn, "Feasibility Study of a Pulsed Thermonuclear Reacter," Los Alamos icien. tific Laboratory Report LA-3294-MS i May 1965)

2. G. I. Bell, W. H. Borkenhagen, F. L. Ribe, "Feasibility Sturies of Pulses High-Beta Fusion Reactors." Procetaings of the B.N.E.S. Conference on Nuclear Funon Rewcrors, UKAEA Colham Laboratory, Sept. 17-1 9, 1969, p.242.

3. F. L Ribe, "Economic Considerat ons and Masnetic Energy Storage for High-Beta Pulsos Reactors," Procieding; Inter society Energy Conversion Engineerng Conference. "Linergy 70, "Las Vegas, Nevada, Sept. 21-2E, 1970, j. 10

4. F. L. Ribe, "Parameter Study of a Lygg, Separared-Shock 5 Pinch with Superconducting Inductive Energy storage," Los Alamos Scientific Laboratory Repore, LA-4828-MS, (Decetnher 1971).

5. T. A. Oliphant, "Fuel Burn-Up and Dizece Conversion of Energy in a D-T Plasma," Proced Jings R.N.E.S. Couterence om Nuclear Fusion Reactors, UKAEA Culham Laborato:y, Sept. 17-19, 1969, p. 309.

6. S. C. Burnett, W. R. Fillis, T. A. Oliphant, Ir. F. L. Ribc. "A Reference Theta Pinch Reactor (RTPR)." Los Alamus Scientific Laboratory Repon LA-51 21-MS (December 1972).

7. K. I. Thomassew, "Reversible Magnetit Enetgy Transfer and Storage Systems," Los Alamos Scientific Laboratory Report LA-5087-MS (Novernber 19?2). See also "Magnetic Enetgy" Transfer and Storage Systems " presented at the Texas Sympo:ium on the Tecbnology of Conerolled Thimonuclear Fusion Experimerts and the Eingineering Asperts of Fusion, University of Texas at Austin, November 1972. 
8. T. A. Oliphant, "Flushing and Refucling of a Pulsed Thermonuclear Reactor by Means of a Neutral Gas Layer, " sulmmitted for publication to Nuclear Fusion. (1973).

9. S. C. Burnert, W. R. Eliis, F. L. Ribe, "Parameter Study of it Pulseủ High- $\beta$ Fusion Reactor," Trans ANS 15, 1, p. 36 (June 1972).

10. T. A. Oliphant, F. L. Ribc, T. A. Coultas, "Direct Conversion by Means of High- $\beta$ Magnctic Compression and Expansion," submitted for publication to Nuclear Fusion, (1973).
11. B. Lehnert. "Screening of a lligh-Density Plasma from Neutral Gas Penetration," Nuclear Fusion, 8. 173 (1968).

12. P. J. Persiant, W. C. Lipinski. and A. J. Itatch, "Some Coniments on the Power-Balance Parameters $Q$ and $\epsilon$ as Measures of Performance for leusion Power Reactions," Argonne National Laboratery Report ANL.7932 (1972). 


\section{SUMMARY PLANT DESCRIPTION*}

The reference theta pinch power plant is a pulsedplasma, lithium-cooled fusion reactor with thermal energy conversion. The fuel is a 50:50 atomic percent mixture of deuterium (D) and tritium ( $T$ ). The plasma pulse duration is approximately $0.1 \mathrm{sec}$ and the cycle time is $10 \mathrm{sec}$. Lithiuin is the primary coolant and also breeds tritium. Two alternative thermal energy conversion systems have been designed. One system uses a lithium-sodium-stcam heat transport system and operates at conservatively rated temperatures, giving a state-of-the-art net plant efficiency of $\mathbf{4 0} \%$. The other system uses a lithium-potassium-steam heat transport system with a potassium vapor topping cycle. This advanced system operates at higher temperatures, giving a net plant efficiency of $56 \%$. The output thermal power is 3,600 $\mathrm{MWt}$ for both energy conversion systems. The net output electrical power for the consen'atively rated plant is $1200 \mathrm{MWe}$, and $1700 \mathrm{MWe}$ for the advanced plant.

\subsection{OVERALL PLANT LAYOUT}

A cutaway view of the plant is shown in Fig. 3.1-1. The plasma is formed in an approximatcly toroidal chamber having major and minor diameters of $112 \mathrm{~m}$ and $1 \mathrm{~m}$, respectively. The large major diameter and high aspect ratio control the plant configuration and set the scale for the large plant area. This area is approximately twice that of comparably rated fossil-fueled or nuclear fission plants where the primary energy source is concentrated in a relatively small firebox or core.

The plasma cisambe $i$ is assembled from 176 cylindrical modu'es, each having an outside diameter of $\sim 3 \mathrm{~m}$ and an overall length of $2 \mathrm{~m}$. A module consists of a blanket section for moderating neutrons and breeding tritium, implosion coils for the initial production and heating of the plasma, adiabatic compression coils for heating and compressing the plasma to thermonuclear burn condition, duct systems for coolant flow, and structural material. Each module has separate, externally located energy supplies for energizing the coils. The modular design was chosen to facilitate assembly and maintenance and to alleviate thermal expansion problems.

The RTPR core (plasma chamber, blanket, and coils) is losated beneath grade level in a 2 -m thick concrete biological shield. This shield is lined with stainless steel and serves as a secondary vacuum chamber for the plasma chamber. The lower portion of the shield is a monolithic support structure penetrated only by relatively sma'l vacuum ducts which lead to vacuum pumps in a lower runnel. The upper portion of the shield is an array of vacuum-tight plugs which isolate and proxect the modulchandling tunnel located above the torus Each modul: weighs approximately 70 metric tons and is handled remotely by an overhead crane which traverses the entir: module-handling tunnel. The crane tunnel is also evacuated and compartmented so that the vacuum lock facii:tates the transfer of modules between the evacuated tunnel and the unevacuated storage areas in the center of the ring. All pipe galleries and tunnels are filled with an inert gas to protect the hot niobium pipes.

Capacitor energy storage units for the implosion crils are located in a tunnel immediatcly outside and slightl: below the module ring. Superconducting energy storag: units for the compression coils are located on three tiers inside the torus, and eight fuel-processing units are located in the lower-level tunnel and outside the torus. Four lithium-processing units are located in the upper deck outside the torus.

The thermal energy conversion system shown in Fig. 3.1-1 consists of four turbogenerators located symmctrically outside the torus. A multiple-loop heatexchanger transports thermal energy from the blanket portion of each module to the steam turbines.

Figures 3.1-2 and 3.1-3 are a plan view and an elevation, respectively, of the plant with the conventional (sodium/steam) energy conversion subsystems. Important parameters and characteristics of the plants are listed in Tables 3.1-1 and 3.1-2.

\subsection{DESCRIPTION OF THE REACTOR MODULE}

Each reactor module is a right circular cylinder havin: an inner diameter of $1.0 \mathrm{~m}$, an outer diameter of $\sim 3 \mathrm{~m}$. and a length of $2.0 \mathrm{~m}$. A cutaway view of the module i: shown in Fig. 3.2-1. Each moduie is a self-contained unit, having independent electrical, mechanical, and flow linc connections to the various power plant systems. Therefore, each module can be inserted into or removed from the tons independently of the other modules. The

-T. A. Coultas, P. Davsvardis, A. J. Hatch, ANL. 


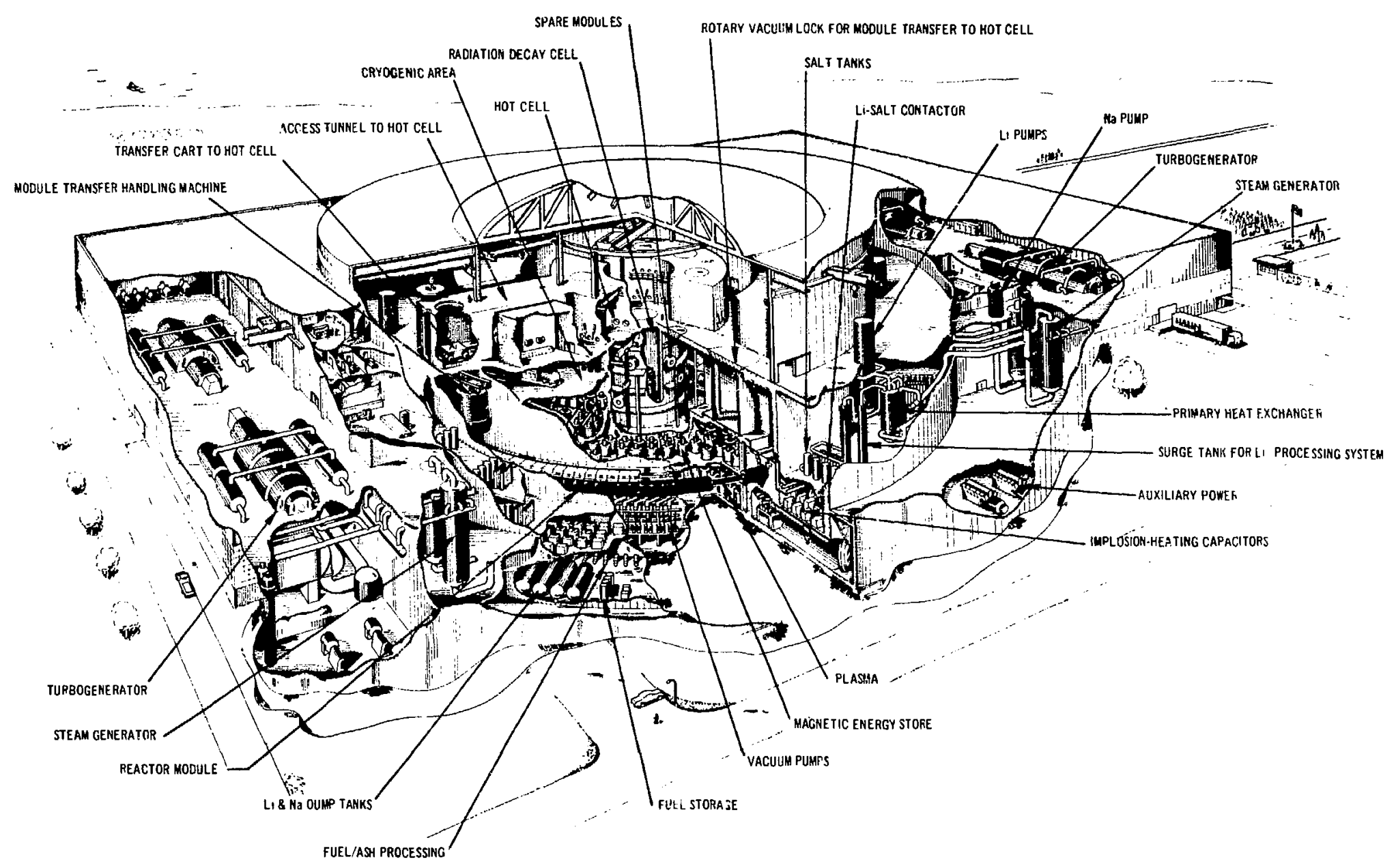

Fig. 3.1-1.

Cutaway view of RTPR power plant. 


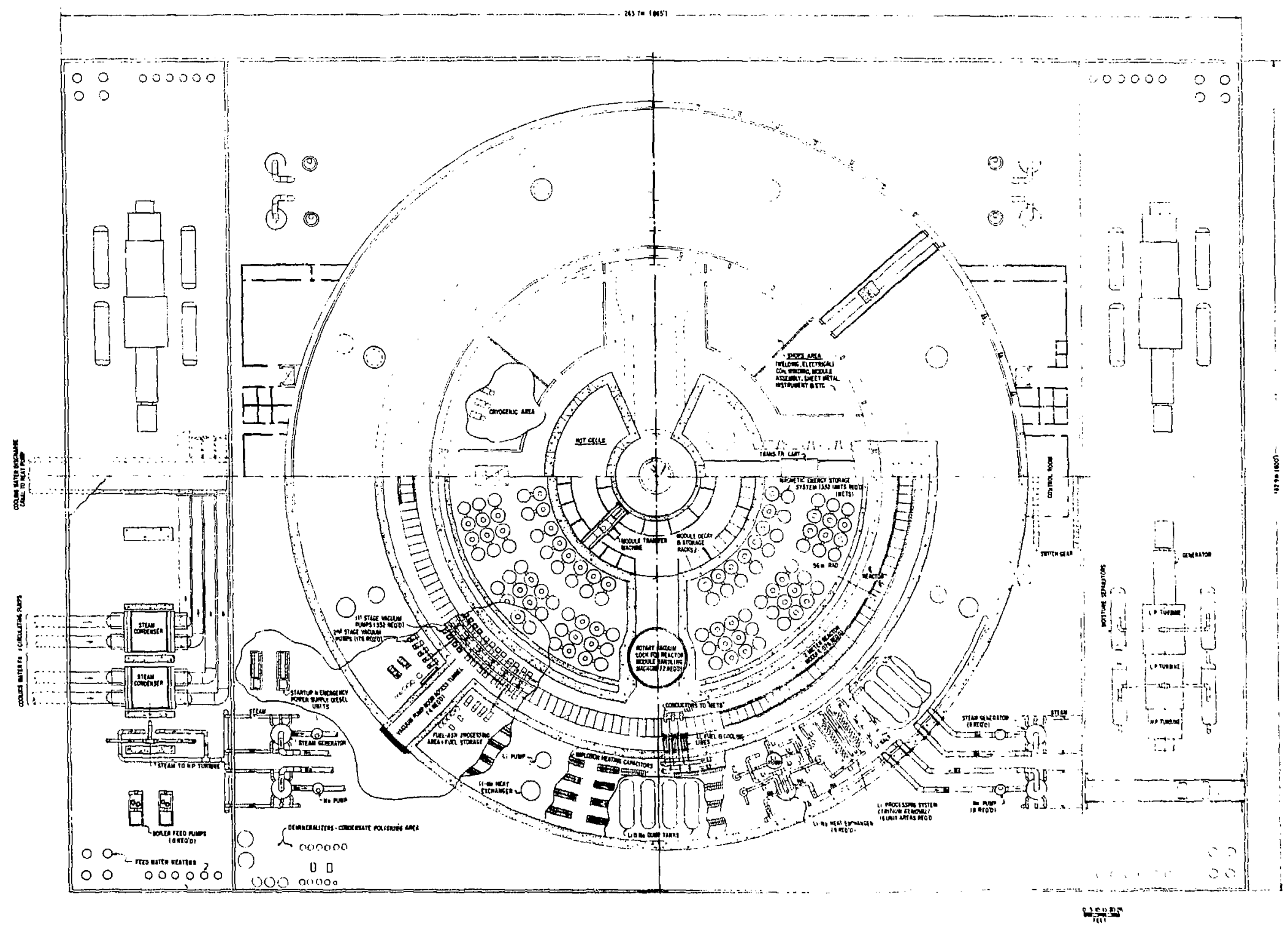

Fig. 3.1-2. 


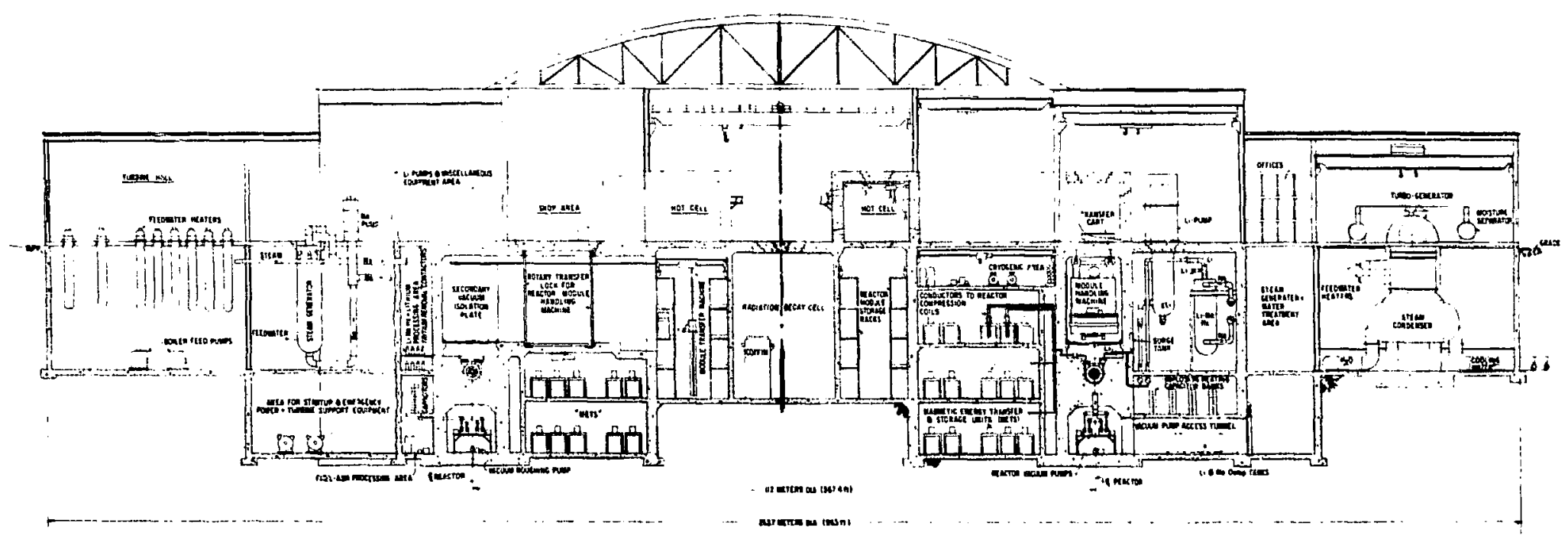

Fig. 3.1-3.

Elevation vicw of RTPR power plant with conventional (sodium-steam) poiver conversion system. 
TABLE 3.1-1

SUMMARY OF PLANT CHARACTERISTICS

$\left(\tau_{c}=10 \mathrm{sec}, \eta_{\mathrm{th}}=0.56\right)$

\section{POWER RATING}

Gross thermal power output, MWt

Gross electrical power output (potassium topping cycle), MWe

Net electrical power output (potassium topping cycle), MWe

Gross overall efficiency (potassium topping cycle), \%

\section{COOLANT LOOP ARRANGEMENT}

Number of primary lithium loops and pumps

Number of potassium boilers

Number of potassium turbines

Number of steam generators

Number of steam turbine-generator units

Number of steam condensers

\section{COOLANT FLOW DATA}

3,600 Total lithium flow, million $\mathrm{kg} / \mathrm{hr}$

25

Total potassium flow, million $\mathrm{kg} / \mathrm{hr}$

7

2,030

Total steam flow, million $\mathrm{kg} / \mathrm{hr}$

3.7

1,700

Lithium temperature, In/out of blanket, $K$.

$1273 / 1400$

Potassium temperarure, turbine inlet, $\mathrm{k}$

1,273

49

Potassium pressure at turbine inlet, $\mathrm{MP}_{\mathrm{a}}$ (psi)

$0.55(80)$
2

2

2

2

2

4
FUEL REQUIREMENTS

Deuterium, kg/day (at rated outpl t)

0.27

0.41

1.04

\section{OPERATING CYCLE}

Plasma preparation time, msec

Plasma thermonuclear burn time, msec

Plasma quench time, msec

Plasma gas-blanket cooling time, sec

Cycle time, sec

10 
TABLE 3.1-2

SUMMARY OF PLANT CHARACTERISTICS

$\left(\tau_{c}=3.0\right.$ sec., $\left.\eta_{t h}=\$ .40\right)$

\section{DIMENSIONS}

Torus major diameter, $\mathbf{m}$

Torus minor diameter (inside), $\mathbf{m}$

Total building plan area, acres

Building height above grade, $m$

\section{POWER RATING}

Gross thermal power out put, MWt

Gross electrical power output, MWe

Net electrical power output

(conventional cycle), MWe

Net overall efficiency

(conventional cycle), $\%$
Maximum building height, $m$

\section{COOLANT FLOU DATA}

112 Tutal lithium flow, million $\mathrm{kg} / \mathrm{hr}$

1.0 Total sodium flow, million $\mathrm{kg} / \mathrm{hr}$

202

12 Total steam flow, million $\mathrm{kg} / \mathrm{hr}$

20.6

75 Lithium temperature, In/out of blanket, $K$

$683 / 820$

41 Sodium temperarure, In/out of intermediasc heat exchanger- $1, \mathrm{~K}$

6251795

Steam conditions at turbine inlet, Temperature Pressure

$755 \mathrm{~K} / 16.6 \mathrm{MPa}$

12,000

4.800

FUEL REQUIREMENTS

4,100

Deuterium kg/day (at rated output)

0.97

Tritium, kg/day (at rated output)

1.46

34

Lithium, kg/day (at rated output)

3.36

\section{OPERATING CYCLE}

Number of primary lithium loops and pumps

8

3

Number of primary heat exchangers

Number of secondary sodium loops and pumps

8

8

Number of secondary steam generators

Number of turbine-generator units

4

8
30

70

Plasma thermonuclear burn time, msec

30

Plasma gas-blanket cooling time, sec

I

Cycle time, sec

3
Number of steam condensers 


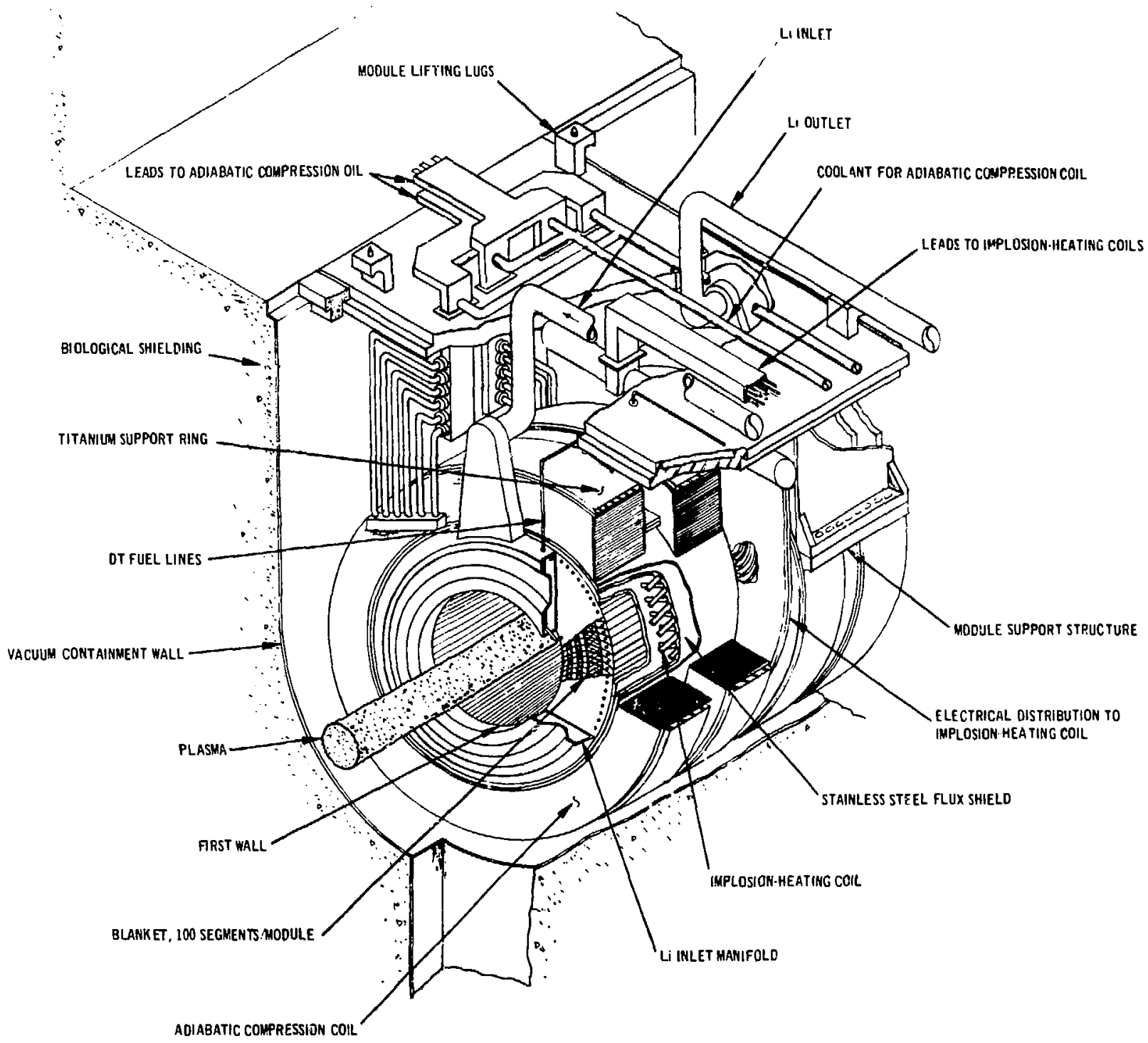

Fig. 3.2-1.

Cutaway view of a 2-meter-long RTPR module. 

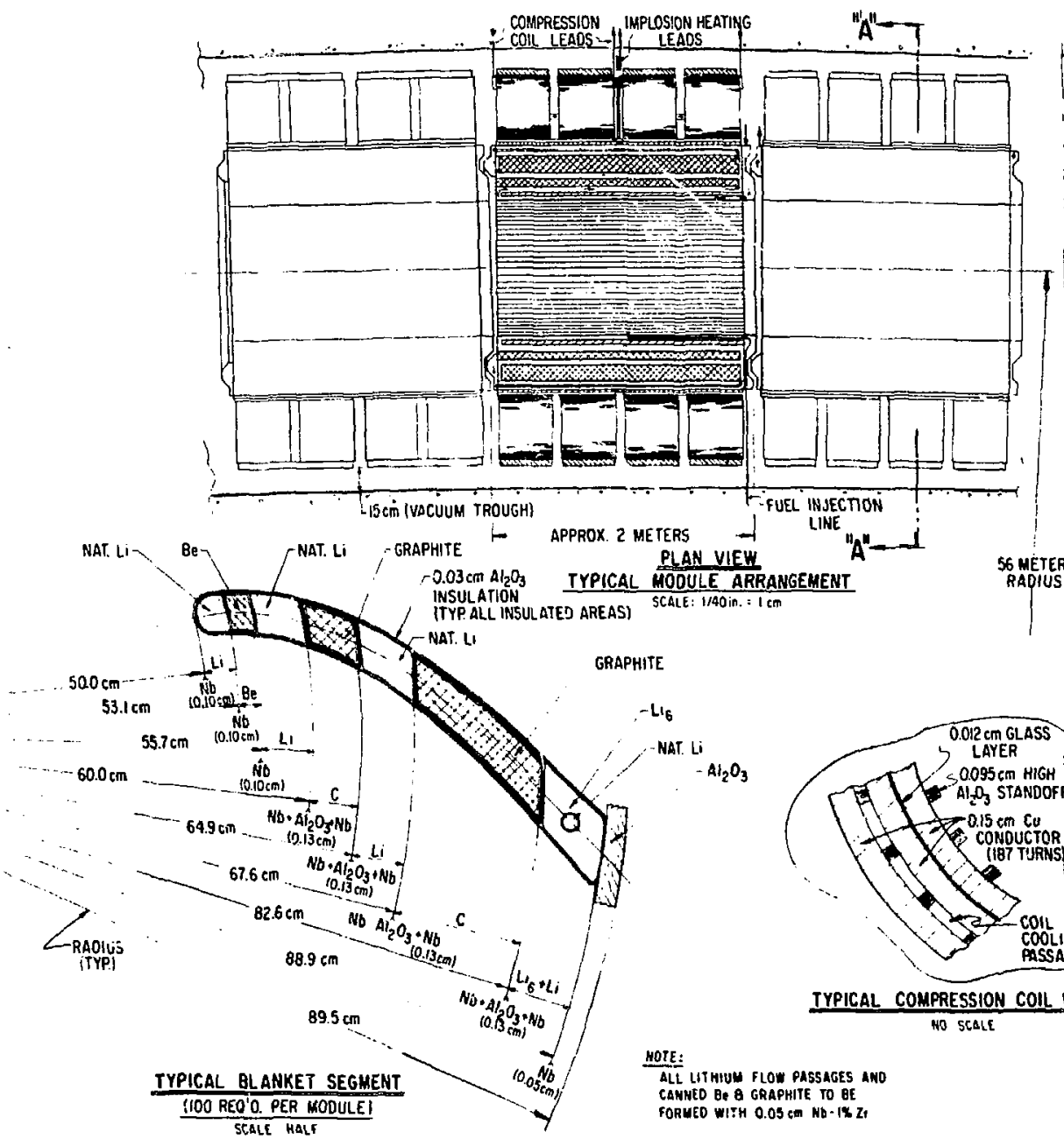

PLAN VIEY

INE "An $A$

MOOULE ARRANGEMENT

APHITE

$-L_{6}$ Nar Li $-\mathrm{Al}_{2} \mathrm{O}_{3}$

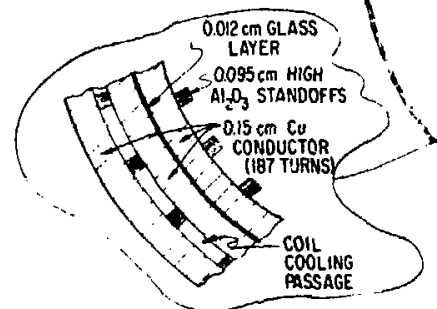

IYPICAL COMPRESSION COIL WIRING Ho scale

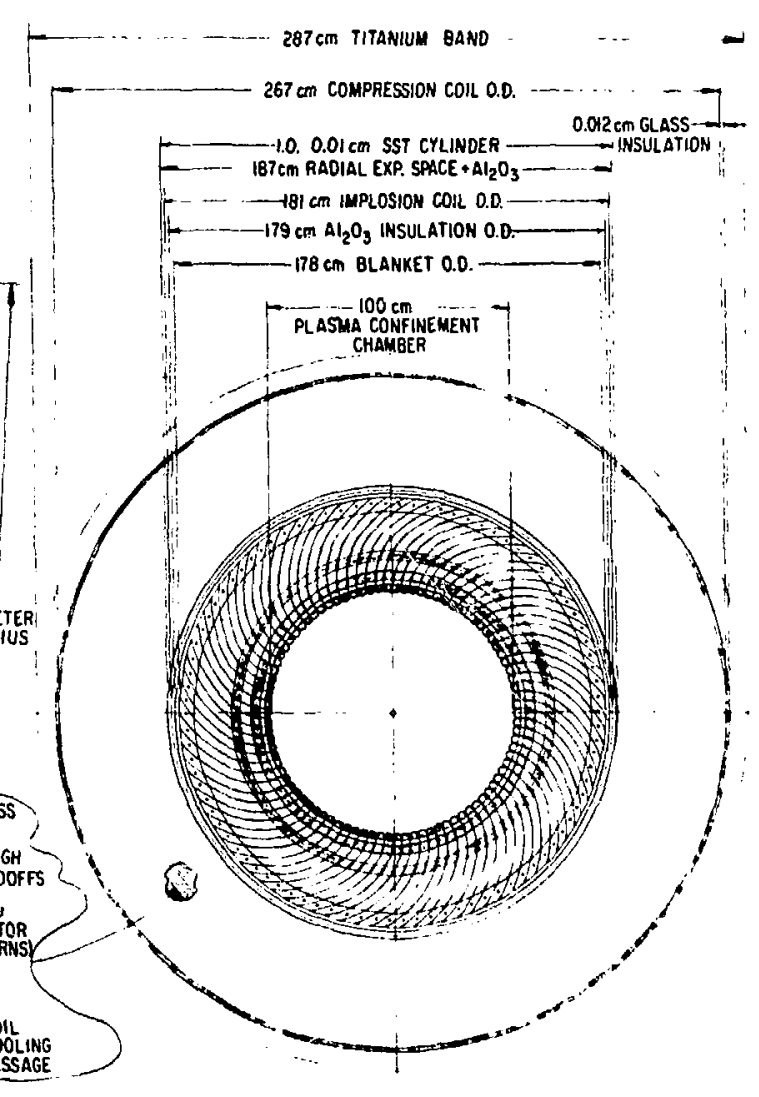

MORE:

ALL LTHHUM FLOW PASSAGES AMO

CANMED BE Q GRAPPITE TO BE
FORUED WITH $0.05 \mathrm{Cm}$ HB- $18 \mathrm{Z}$ 
modules are mounted end-to-end with a small separation for vacuum pumping and thermal expansion at each interface.

Many details of the modules, including the blanket and the coil structures, are shown in Fig. 3.2-2. The innermost 1-m diameter surface represents the first wall that surrounds the plasma. With this first wall as a reference point and proceeding outward radially, the major cylindrical zones of a module are as follows:

1. A $0.10-\mathrm{cm}$-thick first wall of $\mathrm{Nb}-1 \% \mathrm{Zr}$ alloy coated with a $0.03 \mathrm{~cm}$ rhickness of $\mathrm{Al}_{2} \mathrm{O}_{3}$ insulator.

2. A $38-\mathrm{cm}$-thick blanket of alternate layers of niobium, natural lithium, beryllium, graphite, and lithium-6.

3. A $0.5-\mathrm{cm}$-thick layer of $\mathrm{Al}_{2} \mathrm{O}_{3}$ insulator.

4. A 1-cm-thick layer of implosion coil windings made of copper and potted in an inorganic electrical insulator.

5. A $3-\mathrm{cm}$ space for radial thermal expansion.

6. A 0.01-cm-thick stainless steel magnetic shield.

7. A 40-cm-thick zone of compression coil windings made of copper and interspersed with inorganic insulating material and coolant.

8. A $5-\mathrm{cm}$-thick support hoop of titanium.

The blanket is radially segmented to divide the large azimuthal voltages and to minimize eddy-current losses when the implosion field is increasing. Each of the 100 segments is canned in $\mathrm{Nb}-1 \% \mathrm{Zr}$ alloy and coated with a ceramic insulator. Segments are curved to reduce neutron streaming through the niobium alloy cans.

\subsection{DESCKIPTION OF PLANT SYSTEMS}

To facilitate a logical and self-consistent approach to the RTPK engineering design, the reactor system has been divided into the following ten systems according to function:

- Implosion (Shock) Heating System

- Plasma Confinement and Compression System

- Magnetic Energy Transfer and Storage System.
- First-Wall System

- Blanket System

- Biological Shielding System

- Fue! Supply and Removal System

- Fuel-Ash Processing System

- Lithium Processing System

- Energ $y^{\prime}$ Conversion Systein

The first wall represents one of the more crucial problem areas of the RTPR design and for analytical emphasis is considered as a separate system. Figure 3.3-1 gives a schematic outlay of the ten RTPR systems, indicating clearly the interreiationship and interfacing between each. Chapter 4 presents a detailed description and analysis of each of the ten RTPR systems and Chapter 6 summarizes the means by which these ten systems will be assembled, tested, operated, and maintained. A brief summary description of each system is given below.

\subsubsection{Implosion Heating System}

The implosion heating system provides a theta-pinch implosion field of $13.8 \mathrm{kG}$ having a characteristic risetime of $\sim 0.1 \mu \mathrm{sec}$ and a duration of $\sim 10 \mathrm{msec}$. The implosion heating coil (IHC) is wound diagonally as a double layer of copper strap on a cylindrical shell form and is potted in an insulating ceramic material. The IHC is mounted between the blanket and the adiabatic compression coils, where it is protected from excessive radiation damage. Energy for the IHC is provided by a capacitor bank and

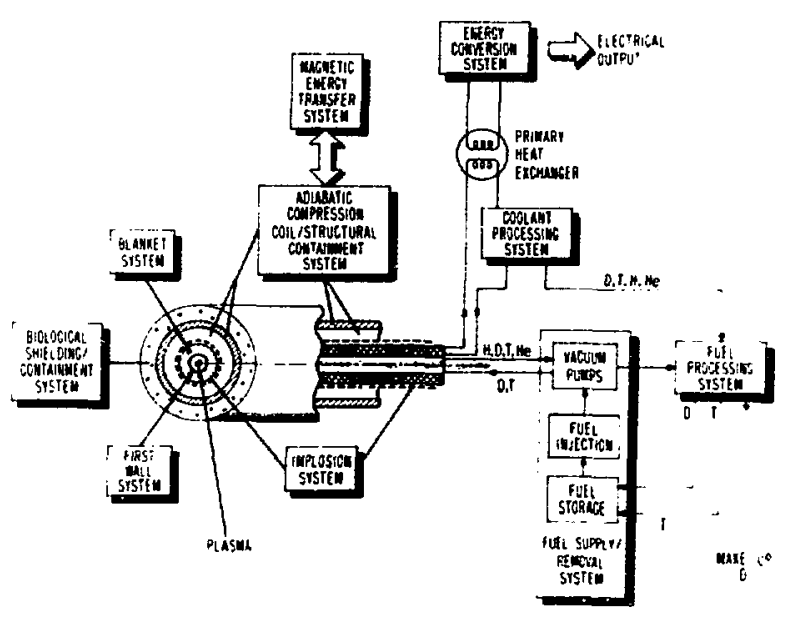

Fig. 3.3-1. Schcmatic diagram of RTPR systems. 
associated spark-gap switching gear located in a tunnel just outside and slightly below the module ring. The pulse-shaping and staging circuitry for the implosion heating system has not been developed, although work currently in progress at LASL' with respect to the Physics Test Reactor will be invaluable in providing a workable system.

\subsubsection{Plasma Confinement and Compression System}

The plasma confinement and compression system provides a theta-pinch compression field of $110 \mathrm{kG}$ having a characteristic risetime of $\sim 31 \mathrm{msec}$. The compression field is staged unto the implosion ficld $(13.8 \mathrm{k}(;)$ and is held for $70 \mathrm{msec}$ at $110 \mathrm{kC}$. After a $\sim 31 \mathrm{msec}$ characteristic decay time to $32.2 \mathrm{kC}$; to quench the thermonuclear bum, the compression ficld is programmed to decay parabolically to zero during the gas-blanket cooling phase. To interface with the implosion-heating pulse. the adiabatic compression pulse must be initiated approxjmately at the midpoint of the implosion-heating pulse. The adiabatie compression coil (ACC) is formed of copper strap wound in multiple-layer, single-turn, pancaks corils with interspersed ceramic spacers and coolant passages. The ACC operates near room temperature. Finergy for the ACC is provided by a superconducting energy storage and switching system, such as a set of variable-inductance coils or a homopolar generator (Sec. 4.3).

\subsubsection{Magnetic Energy Transfer and Storage System}

The Mits system stores, transfers, and returns the large amount of magnetic energy used in the adiabatic compression system. During the 110 lok ; flat-top portion of the compression ficld, the energy stored in the compression field per meter of plasma length is $176 \mathrm{MJ} / \mathrm{m}$. The energy transfer at the beginning and end of the pulse oceurs within $\sim 31$ msec. The encrgy transfer must occur with high efficiency (>98\%) since the amount of energy transferred is $\sim 4$ times the (useful) fusion encrgy developed per pulse. One candidate system to perform this demanding task is a nested set of three superconducting spherical coils, the inner two of which are mechanicalty linked but magnetically decoupled. By rotating these nwo coils $180^{\circ}$ with respect to the fixed ou*er coil, over $60 \%$ of the total stored magnetic cnergy of the system can be transferred to the ACC: by means of a transfomer. $A$ subsequent $180^{\circ}$ rotation (in either cirection) will return the transferred energy to the spherical coil systent. Work done by the cxpanding plasma against the compression field during the quench phase of the R'THR power cycle more than compensates for the ohmic losses incurred in the ACC during the adiabatic compression and plasma couling stage

Another candidate system is a superconducting homopolar generator with inertial energy storage. This system is considered briefly in Sec. 4.3. and can be used either as a transfer capacitor between the storage and load coils or as a major component in the energy storage and staging system.

\subsubsection{First-Wall Systiem}

The first-wall system is the insulator-metal composite of $50-\mathrm{cm}$ radius which serves as the plasma-blanket interface. It is required to withstand the direct particle and radiation bombardment (14-MeV neutrons. hot neutral atonts, and bremsstrahlung) from the plasma as well as back-scattered neutrons and gamma radiation from the blanket without exceeding the erosion limits set by intrinsic requirements of structural integrity ind plasma contamination. It must withstand the $\sim 2-\mathrm{kV} / \mathrm{cm}$ voltage pulse during the $\sim \mathbf{0 . 1} \mu \mathrm{sec}^{2}$ risetime of the implosion heating pulse. The first-wall composite must also be chemically comparible with liquid lithium and be stable in the chemically reducing environment. Finally. the first wall must have acceptable neutronic properties. a high melting point, and adcquate thermal conductivity to accomodate the large volumetric energy deposition. For the present design the first wall is made of 10 .lo-cm-thick niobium. coated with a $0.03-\mathrm{cm}$-thick layer of $\mathrm{Al}_{2} \mathrm{O}_{3}$.

\subsubsection{Blanket System}

The blanket system moderates the 14-MeV neutron thux from the plasma, converts the neutron kinetic cnergy to thermal energy, and breeds tritum. The blanket section of each module is an assenbly of 100 radial segments which make up a series of cylindrical shell zones of alternate layers of lithium, beryllium, graphite, and lithium-6: all regions are canned in niobium and coated with a ceramic insulator. The lithium coolant flows only when the magnetic field is nearly zero. During this flow interval approximately $101 \mathrm{MJ} / \mathrm{m}$ of thermonuclear energy must be transported out of the blanket. Surge tanks are provided so that the lithium coolant pumps can operate continuously and at a uniform power level throughout the power cycle.

\subsubsection{Biological Shielding System}

The biological shiclding system is required to attenuate the neutron and ganma radiation emanating from the 
reactor core to levels that are acceptable for eithe: limited or unlimited access to galleries, decks, tunnels, and other regions adjacent to the reactor module, module handling equipment, and storage areas. Supplementary shielding is required around coolant, vacuuni, and electrical passthroughs. Temporary shielding is required for general accessibility and maintenance of auxilliary subsy'stems such as primary heat exchangers, tritium processing equip* ment, and lithium processing equipment. For most purposes $\sim 2 \mathrm{~m}$ of ordinary concrete appears to provide adequate shielding.

\subsubsection{Fuel Supply and Removal System}

The fuel supply and removal system supplies a highpurity D-T mixture at the proper pressure to the plasma chamber at the teginning of each cycle and regulates the gas pressure in regions of the reactor external to the plasma during the D-T burn, quench, and cooling phases of the cycle. The s:-stem also flushes unburned fuel and contaminating burn products from the plasma chamber at the end of each cycle. The RTPR powar cycle is controlled mainly by the pumping speed of a continuousflow, vacuum system using Roots blowers as primary pumps and compressors as secondary pumps.

\subsubsection{Fuel Processing System}

The fuel processing system continuously separates helium and other impurity products from the D-T fuel flowing from the plasma chamber. A multiple-stage cryogenic fractional distillation column system is used.

\subsubsection{Lithium Processing System}

The lithium processing system removes bred tritium from both the ${ }^{6} \mathrm{Li}$ and ${ }^{7} \mathrm{Li}$ portions of the blanket on a continuous basis and maintains the lowest possible tritium inventory in the blanket and coolant stream. A molten salt contactor system is used.

\subsubsection{Energy Conversion System}

The energy conversion system transports thermal energy from the blanket to a suitable heat exchanger system in which steam or another working medium is generated to operate turbogenerators. This system must permit only a minimal amount of tritium to diffuse into the steam, because tritium recovery from steam is known to be unfeasible. Two alternative systems are used. One system involves a conventional steam cycle using a lithium-sodium-steam cycle at moderate temperatures $(820 \mathrm{~K}$ maximum lithium temperaturel, which gives a modest plant thermal efficiency of $40 \%$. The other system combines a potassium vapor topping cycle with a lithiumpotassium-steam heat exchanger chain at high temperanures (1400 $\mathrm{K}$ maximum lithium tempenture), which gives a more desirable overall plant efficiency of $56 \%$. (See Table 3.1-1 and 3.1-2)

\section{REFERENCES}

1. F. L. Ribe (Comp.). "Propiosed Experiments on Heating, Staging. and Stabilization of Theta Pinches". Los Alamos Scientific Laboratory LA-5026-P (September 1972). 


\section{4. )ESIGN DESCRIPTICN}

\subsection{IMPLOSION HEATING SYSTEM*}

in the staged theta-pinch concept, implosion hearirig is done by high-voltage circuits whose energy content is only a small fraction of that required by the cotal system. The magnetic energy associated with adiabatic compression is preponderant and is contained in a coil with slow risetime. The mixture of hating by implosion and adiabatic compression can be adjusted to give desired radial compression ratios of about 0.20 . This process of staged heating is calculated on the basis of plasma sheaths driven by high-voltage transmission lines, followed by equilibration of ion and electron temperatures and arliabatic compression. This section describes the design aspects and related physics of the implosion heating system.

\subsubsection{System Requirements}

The implosion heating coil is located at a radius of $\sim 90 \mathrm{sm}$ and must provide a magnetic field cqual to $\sim 14 \mathrm{kG}$ for $\sim 10 \mathrm{msec}$ with a riserime of $\sim 0.10 \mu \mathrm{sec}$. Furthermore, the inaplosion field must interface with the slowly rising compression field, as indicated on Fig. 4.1-1. Figure 2.1-1 illistrates the implosion process diagrammatically. The initially cold plasma has a radius equal to that of the first wall $(50 \mathrm{~cm})$ and is compressed to a factor of $\sim 0.7$ of the first-wall radius: the ion temperature after implosion heating is $2.3 \mathrm{keV}^{\prime}$. During the implosion stage the ion sheath moves radially inward with a velocity of $\sim 20 \mathrm{~cm} / \mu \mathrm{sec}$ and therefore induces an azimuthal electric field at the first wall equal to $2.0 \mathrm{keV} / \mathrm{cm}$. The energy required by the implosion-heating stage is $1.7 \mathrm{MJ} / \mathrm{m} / \mathrm{pu}$ se of which approximately $0.3 \mathrm{MJ} / \mathrm{m} /$ pulse appears as plasma internal energy.

\subsubsection{Physics of the Implosion Heating Process}

Figure 2.1-2 illustrates the process of magnetic-piston or implosicn heating being considered. Two feed slots permit entry of magnetic energy generated by the implosion heating coil. Ideally, this arrangement dri res a magnetic field parallel to the axis of the system. The field advances radially inward on a field-free plasma, and a thin

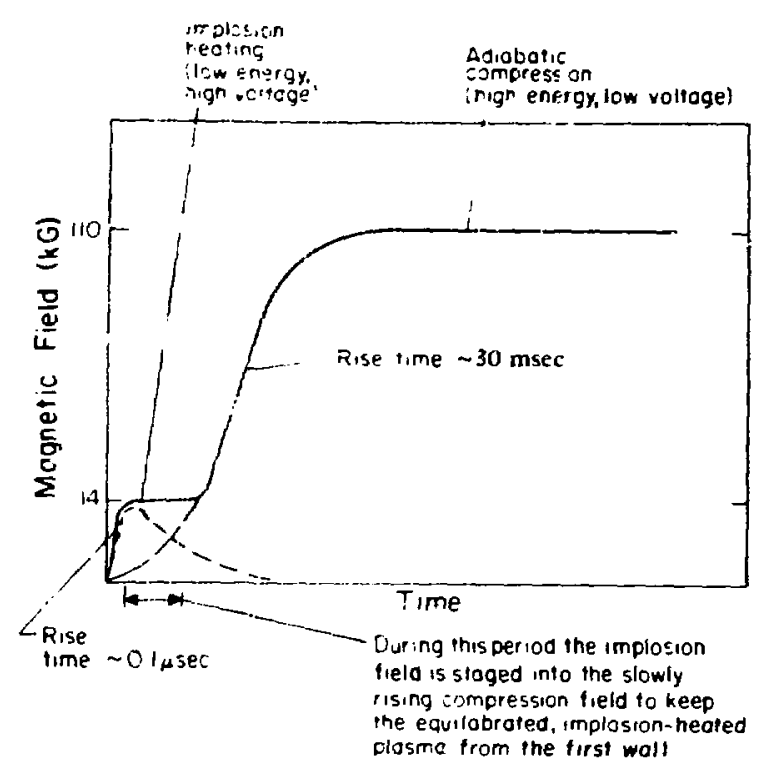

Fig. 4.I-1.

Schematic diagram sbowing the staging of the implosicu antì compression fields.

ion shearh sejrarates the plasma and the magnetic field throughout the implusion stage.

On the time scale of interest the classical conductivity of the theta-pinch plasma in its initial state is sufficiently. large to exclusts completely the magnetic field between the coil and tixe plasma. Under these conditions the magnetic field acts as a massless piston to push the plasma toward the aris. The detailed response of the plasma to the piston depends on the mean free path of the ions in the plasma. If the mean free path is much shorter than the radius, $b$, a shock forms and propagates radially inward from the mignetic piston through the plasma, greatly increasing the plasma internal energy. In addition, the shock-hicated plasma has significant kinetic energy of motion toward the axis; this kinetic energy must eventually be: converted to plasma heat when the inward

"J. Marshall, F. L. Ribe, LASL. 
motion is stopped by compression near the axis. However, experimental and theoretical evidence suggests that the ion mean free path is long in theta pinches. In this case, ions should simply bounce off the imploding piston with twice the piston velocity if the piston velocity is much larger than the initial thermal velocicy. This kind of pistun heating without the actual formation of a shock plays the same role in plasma heating as a shock and, in fact, is somewhat more effective, since it can be shown to make the most efficient use of a given power supply.

The effect of either process is to heat the plasma more for a given compression ratio than would be done by adiabatic compression. This process is nonisentropic and can be viewed in terms of increasing the plasma entropy. The temperature achieved by nonisentropic heating is limited, however, by the speed at which the imploding piston can be driven, which in turn amounts (o) the practical technological limit on the voltage applied to the coil. Nonisentropic heating must be folluwed by some adiabatic-compression heating to achieve thermonuclear temperatures at sufficiently high plasma densitics to be of practical interest. The implosion heating circuits use high voltage and are relatively expensive per unit of energy. However, the energy used is small because of the low magnetic ficld. Additionally, the relative amounts of implosion and compression hearing can be adjusted so that the plasma fills an appreciable fraction of the vacuum volume. This is necessary for reactor power balance and desirable for MHD stability in toroidal systcms. 1,2

The fundamental scaling equations which describe the implosion heating process are developed in Appendix $A$. The case of simple implosion heating (Appendix A.1) is shown to be of optimal conditions with respect to a programmed implosion heating scheme. To obtain lower compression for a given implosion (shock) heating field, a programmed implosion heating scheme which allows for free expansion of the ions (Appendix A.2) is used. Equations 4.1-1 and 4.1-2 summarize the results of the analyses given in Appendix $A$ and state the fundamental design relationships for the implosion heating system. The units are the same as those used in Sec 2.1 .

$$
\begin{aligned}
& \mathrm{V}_{\mathrm{S}}=1.27 \mathrm{P}_{\mathrm{A}}^{1 / 2} \mathrm{~b}\left[\mathrm{kT}_{\mathrm{SH}}\right] \\
& \mathrm{B}_{\mathrm{S}}=2.22 \mathrm{P}_{\mathrm{A}}^{1 / 2}\left[\mathrm{kT}_{\mathrm{SH}}\right]^{1 / 2}
\end{aligned}
$$

$P_{A}$ is the filling pressure at $300 \mathrm{~K}$ in mTorr and $\mathrm{b}$ is the first wall radius in $\mathrm{cm}$. The free-expansion value for $x_{\mathrm{SH}}$ (0.76) has been used. The inward velocily of the ion sheath is given by

$$
u_{S}=1.29 \times 10^{7}\left[\mathrm{kT}_{\mathrm{SH}}\right]^{1 / 2}
$$

\subsubsection{Design Aspects of Implosion Heating System}

4.1.3.1 Electrical Interaction of Implosion-Heating Coil with Other Systems. The implosion heating coil is energized with an overall risetime $(\sim 0.1 \mu \mathrm{sec})$ that results in : very large time rate-of-change of magnetic field. The hight rate of change of magnetic inductance may couple the enclosing compression coil, which by trarisformer action. could lead to a voltage appearing across the turns of the compression coil. Since the turns ratio is 1 to 187 , the inuplosion heating voltage $(650 \mathrm{kV})$ can in principle be multiplied to $-120 \mathrm{MV}$ on the open-circuited compres sion coil. A ralated effect is the transmission line process. which may uccur on the long length of the compression, coil; if the 187 -turn coil is represented by a continuou: turn circuit element, the length is about $1.4 \mathrm{~km}$. Tht transmission time for this length is $\sim 4 \mu$ sec and exceed: the risetine of the shock-heating voltage by a factor of $\sim 40$. This impedance mismatch could increase the already. high voltage induced between turns on the compression coil.

Another potential problem is the "shorting" of the implosion heating coil by a continuous, external loop of conducting material. If the ends of the compression coi' are capacitively grounded during the firing of the implo sion heating circuit, the compression coil would, in fact act as an externally shorted turn.

Other problems can be related to the interaction. between the implosion-heating and adiabatic-compression systems. Until experimentation and more detailed circuit analyses can elucidate the high-roltage, fast-risetime effects, two methods of dealing with the situation have been included in the design. The first technique, and the one adopted in this design, is to shield the compression coil from the implosion heating field by a metallic hoop placed between the implosion heating and compression coils. The metal shield is thin enough to permit penetration by the compression fie!d but thick enough to shield the cumpression coil from the implosion heating field. The area for flux return between the implosion heating coil and the metal hoop has not been optimized. As this area is increased, the energy requirements of the 
implosion heating system diminish, although the energy requirements of the cornpression coil increase.

Other possibilities of alleviating the electrical interaction between the implosion heating and compression coils are being considered. The turns in the compression coil could be put in series; the compression coil turns could be capacitively shorted outside the radiation shielding; and the compression coil could be adequately insulated to withstand the voltages induced during the implosion heating.

4.1.3.2 Construction of Implosion Heating System. The relative position of the implosion heating coil (IHC) with respect to other components of the core is indicated on the reactor core cross-section in Fig. 3.2-1. The IHC has a radius of $\sim 90 \mathrm{~cm}$ and is separated by thermal insulation fror 1 both the reactor blanket and the adiabatic compression coil. Because of the time scale associated with the operation of the $I H C$, the conductors are "litzed" to reduce skin effects. Internal gaseous cooling will be provided to remove transport current losses and gammaray heating. Because of the vacuum environment and high gamma-ray field, inorganic electrical insulation totally enclosing coils and leads must be used; glass, plasmasprayed ceramic, and swaged ceramic powders are being considered.

The voltage and coolant passthroughs to the IHC must be located at the end(s) of each coil sector (length $\sim 1$ to $2 \mathrm{~m}$ ). Since the electrical connections to the fast, lowinductance power supply (capacitors, spark-gaps, crowbar circuit) must traverse the adiabatic compression field. The IHC leads must present a minimum profile to the adiabatic compression field; eddy current losses and mechan:cal strain in the IHC passthroughs must be minimized.

A simplified design for the IHC is presented in Fig. 4.1-2. Although many design details are yet to be resolved, the scheme indicated in Fig. 4.1-2 represents a useful calculational model. The current passing through each separate conductor adds vectorially in the $z$ direction. The net current, therefore, has a purely theta orientation and the desired $\mathrm{B}_{2}$ field is thereby derived.

Each copper conductor shown in Fig. 4.1-2 is attached to a coaxial cable leading through the shield to the power supply. The shock coil power supply (high-voltage capacitors) is situated immediately adjacent to the vacuum chamber. The coaxial cables leading from the shock coils to the capacitive power supply are insulated with glass or ceramic and subsequently "potted" into a ceramic mold. Each coil is an integral piece with the module assembly; wis an a module is exchanged in the torus the implosion coil, coolant, and electrical leads are also changed as an
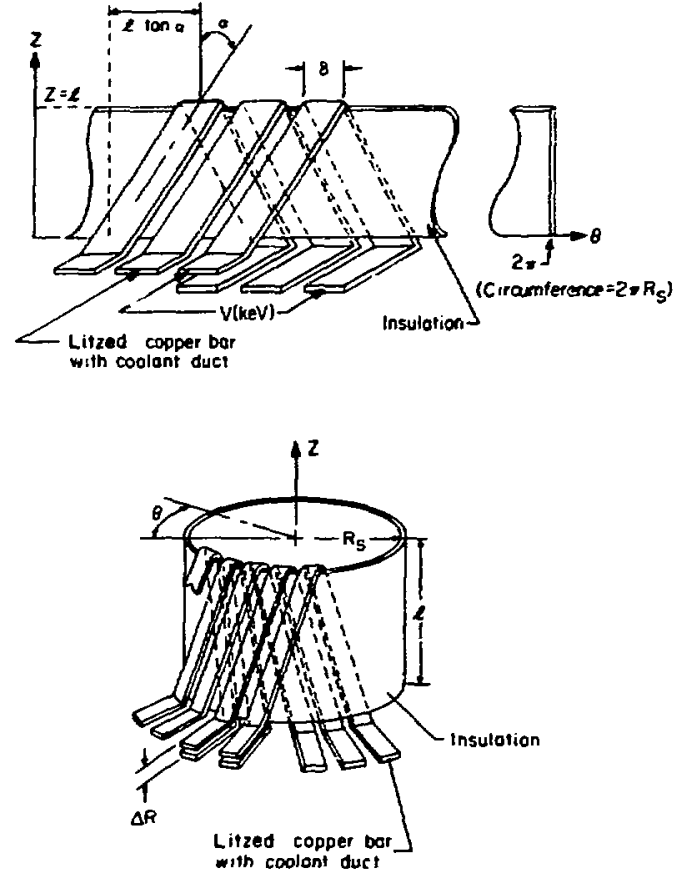

$L=$ lengih of implosion heasing coil $R_{S}=$ radius of implosion heoting coil

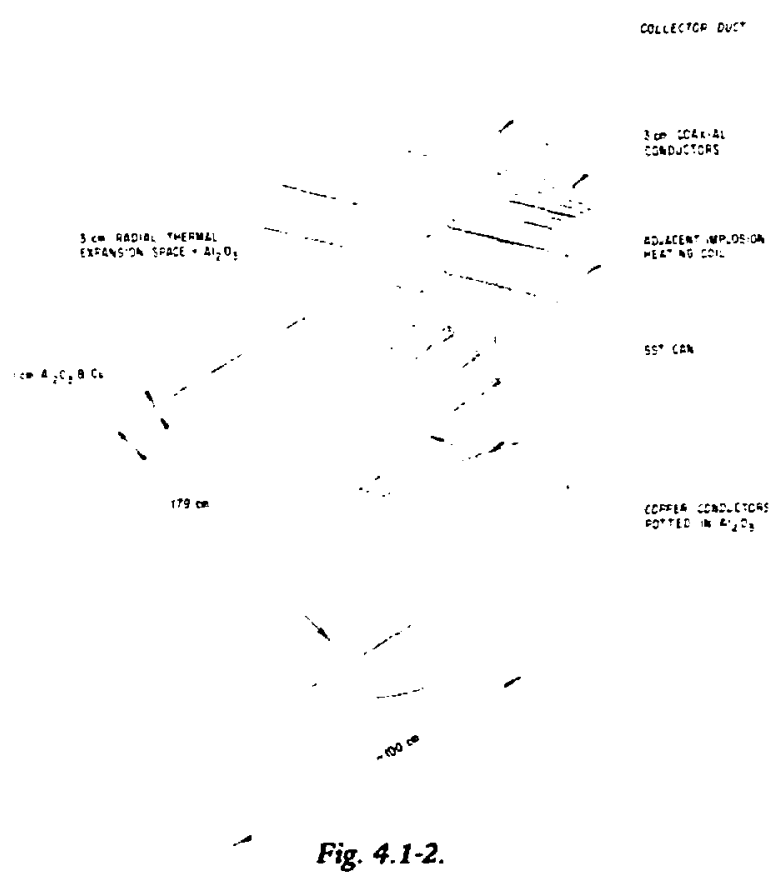

Proposed construction scberse for implosion beating coil. 
integral unit. The assembly of this module is shown schematically in Fig. 3.2-1.

Referring to Fig. 4.1-2 for dimensional notation,

$\mathrm{V} / \mathrm{V}_{\mathrm{S}}=\frac{\ell \tan \alpha}{2 \pi \mathrm{R}_{\mathrm{S}}}$

where $V$ is the voltage across each fractional turn of the IHC $(\sim 60 \mathrm{kV})$ and $V_{s}=650 \mathrm{kV}$ is the total azimuthal voltage. For $\ell \sim 100 \mathrm{~cm}$ and $R_{s} \sim 90 \mathrm{~cm}$, the angle $\alpha$ becomes $\sim 14^{\circ}$.

A detailed analysis of the implosion heating power supply and associated pulse shaping network for the RTPR has not been made. Recent experiment at LASL, however, on heating, staging, and stabilization of theta pinches $^{3}$ will be invaluable in developing a clearer picture of the electrical requirements of the IHC. For instance, Fig. 4.1-3 illustrates a circuit which uses a Marx generator to fast-charge through the inductance of the Marx and the connecting coaxial cables. The capacitance in the pulse shaping network contributes to ho!ding-up of the current through the plasma load. Circuit analyses are presently being made of the system depicted in Fig. 4.1-3 which, of course, include the effects of the varying inductance of the imploding plasma $\left(\mathrm{L}_{\mathrm{p}}(\mathrm{t})\right)$. Additionally, component development (capacitors, switches, transmission lines) is in progress at LASL to provide a system capable of supplying implosion aging current to a four feed-slot coil at a nominal $125 \mathrm{kV}$ per feed point ( $500 \mathrm{kV}$ total). Although the related electrical requirements of the RTPR dre more stringent, experience gained from on-going implosion work at LASL will be valuable in satisfying the electrical requirements of the RTPR.

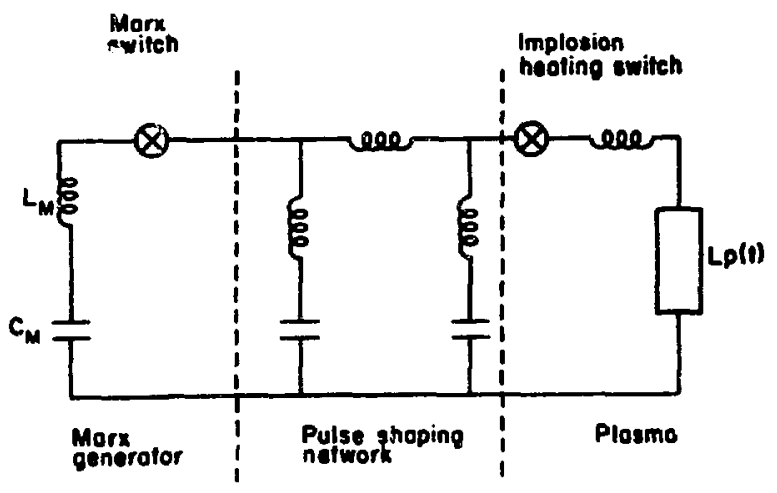

Fig. 4.1-3

Schematic diagram of implosion beating electrical circuit.

\section{REFERENCES}

1. R. F. Gribble and F. L. Ribe, "Low-Compression Theta Pinch with Separated Shock Heating," Los Alamos Scientific Laboratory Report LA-4194MS (j une 1969).

2. J. P. Freidberg and R. L. Morse, Proc. Second Topical Confer ence on Pulsed High-Beta Plasmas, Garchirg, Germany, Max-Planck Institute fur Plasmaphysik Report IPP 1/127 (1972) p. 139.

3. F. L. Ribe, (Comp.), "Proposed Experiments on Heating, Staging, and Stabilization of Theta Pinches," Los Alamos Scientific Laboratory Report LA-5026-P (September 1972).

\subsection{PLASMA CONFINEMENT AND COMPRESSION SYSTEM*}

\subsubsection{System Requirement}

The adiabatic compression coil, associated electrical passthroughs, and structural members comprise the plasma confinement and compression system. The compression coil must provide a magnetic field of $110 \mathrm{kG}$ with a risetime of $\sim 31 \mathrm{msec}$ and sustain the field for $\sim 70$ msec. The field is then reduced to $32 \mathrm{kG}$ with a falltime of $\sim 31$ msec. Thereafter, the magnetic field must be programmed to decrease to zero in 1 to $3 \mathrm{sec}$, while the internal energy of the plasma is transferred by thermal conduction through a neutral gas blanket to the first wall. The plasma radius throughout the plasma cooling stage is to be maintained constant at $46 \mathrm{~cm}(4 \mathrm{~cm}$ from the first wall); the plasma cooling calculations presented in Sec. $\mathbf{2 . 3}$ indicate that $\mathrm{B}_{\mathrm{O}}^{2}$ must decrease linearly in time. Heat transfer calculations given in 4.4 .3 indicate that 1 to $3 \mathrm{sec}$ represent an adequate time scale over which $B_{O}$ is reduced to zero.

An important design requirement of the compression coil is the minimization of eddy current and transport current losses. These lisses, although not reflected in METS efficiency $\eta_{B}$ (Sec. 2.4), must be eventually supplied to the METS.

The plasma confinement and compression system must be designed to withstand the pressures generated when the coil is activated and cycled as well as stabilizing this massive structure. Additionally, the design of the compression coil and associated support structure must permit passage of cooling fiuid and electrical feedthroughs.

-R. A. Krakorvski, F. L.. Ribe, LASL; T. A. Coultas, P. Dauzvardis, ANL. 


\subsubsection{Physics of Adiabatic Compression}

The thermodynamic state of the plasma immediately after the implosion heating stage is described in Sec. 4.1 and Appendix $A$, and represents the initial condition for the adiabatic compression.

During the adiabatic compression the plasma is assumed to behave as a gas with a ratio of specific heats equal to $\gamma=5 / 3$. Using the adiabaric relationship $\mathrm{PV} \gamma=$ constant, the plasma temperature after adiabatic compression, $T_{0}$, and the temperature after implosion heating, $\mathrm{T}_{\mathrm{SH}}$, are related to the respective compression ratios by

$\mathrm{T}_{\mathrm{O}}=\mathrm{T}_{\mathrm{SH}}\left(\mathrm{x}_{\mathrm{S}} / \mathrm{x}_{\mathrm{O}}\right)^{2(r-1)}$

Recalling that the density after adiabatic compression, $n_{0}$, is related to the filling density, $n_{A}$, by

$n_{0}=n_{A} / x_{0}^{2}$

and invoking pressure balance, i.e.,

$n_{0} k T_{0}=B_{0}^{2} / 8 \pi$

we obtain the following relationship between $B_{0}(k G)$ and $k T_{O}(k e V)$ is obtained.

$B_{0}^{2}=5.71 P_{A}\left[k T_{0}\right] / x_{0}^{2}$

Combining liq. $(4.2-4)$ with the implosion heating relarionships summarized in Sec. 4.1 yields the following important design relationships.

$$
B_{S}=4.30\left\{\left[B_{0}\right]^{2 / 5} E_{\theta} /\left[\mathrm{kT}_{0}\right]^{1 / 2}\right\}^{5 / 7}
$$

$$
E_{\theta}=0.244\left[\mathrm{x}_{0}\right]^{? / 3}\left[\mathrm{kT}_{0}\right]^{1 / 2} \mathrm{~B}_{0}
$$

wfiere the units are $\mathrm{kG}, \mathrm{keV}$, and $\mathrm{cm}$. Table 2.4-1 summarizes important plasma parameters applying during the adiabatic compression process and were derived from these scaling relationships.

\subsubsection{Compression Coil Design}

4.2.3.1 Electrical Design of Compression Coil. The current in a pulsed solenoid has two components. as shown in Fig. 4.2-1A. The transport current, $\mathrm{j}_{\mathrm{T}}$, furnishes the magnetic field $B$ in the coil bore according to Ampere's law $\nabla \times \vec{B}=0.4 \overrightarrow{\pi j}$. The eddy current density, $\mathrm{j}_{E}$, arising from the time variation of magnetic field forms closed paths in the windings and does not contribute to the m:gnetic field on axis. The latter is governed by Faraday's law of induction, $\nabla \times \mathrm{E}=-10^{8} \partial \overrightarrow{\mathrm{B}} / \partial \mathrm{t}$. These two components are illustrated in Fig. 4.2-1B and are discussed in detail in Ref. 1.

Transport Current Losses. The energy loss from transport current is the same as for dc current. Using Ampere's law, the transport current density, $\mathrm{j}_{\mathrm{T}}$, is given by

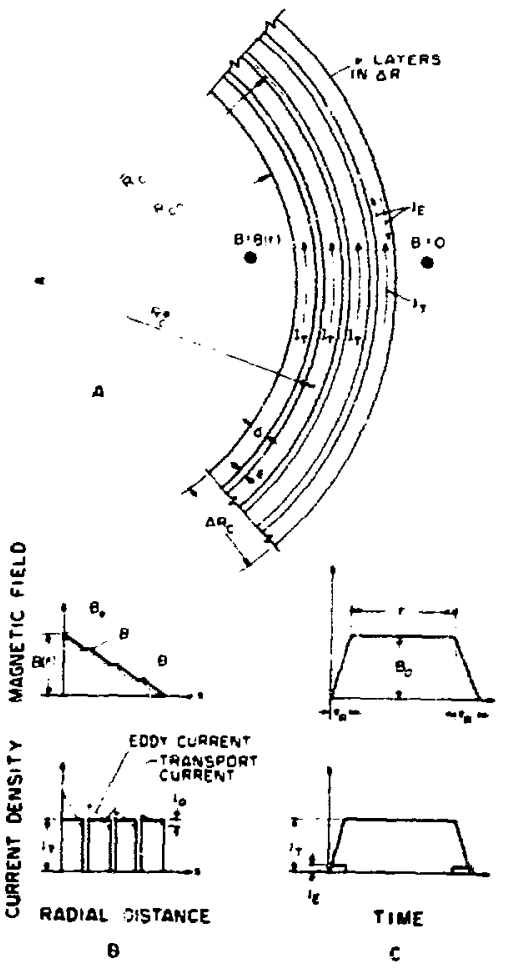

Jig. 4.2-I.

Schematic diagram of compression coil geometry. magnetic ind actance'. and currents.

A. Multifum magnetic compression coil sbowiug transpore aled eddy carrents and sie radii $R_{c o} . R_{c}$. and $R:$

B. Radial distribution of magnetic field. mausport currerif, and eddy currem

C. Time oariation of magnerie field, tratuspors cir. rent. and eddy cormont. 
$\mathrm{j}_{\mathrm{T}}=\mathrm{B}_{0} / 0.4 \pi \lambda \Delta \mathrm{R}_{\mathrm{C}}$

where $\Delta R_{c}$ is the total compression coil thickness and $\lambda$ is the volume filling fraction with respect to the conductor. If $\bar{R}_{c}$ is the mean coil radius,

$\bar{R}_{c}=R_{c o}+\frac{\Delta R_{c}}{2}$

where $\mathbf{R}_{c o}$ is the inside radius of the compression coil. The total joule energy loss per unit length of coil, $\mathrm{w}_{\mathrm{T}}(\mathrm{Cu})$, resulting from the driving current during a flattopped pulse of duration $\tau_{B}$ and risetime $\tau_{R}$, referring to Fig. 4.2-1c and Eqs. (E-17) and (E-18) of Ref. 1, is given by

$$
\begin{aligned}
W_{T}(C u) & =\frac{2 \pi \bar{R}_{c} \eta B_{O}^{2}}{(0.4 \pi)^{2} \lambda \Delta \bar{R}_{c}}\left(\tau_{B}+\frac{2}{3} \tau_{R}\right) \\
& \left\{1+\frac{1}{2}\left(\frac{B_{Q}}{B_{0}}\right)^{2}\left(\frac{\tau_{Q}}{\tau_{B}+2 / 3 \tau_{R}}\right) \mid\right. \\
& =4,0 \times 10^{-4} \frac{\bar{R}_{C} B_{0}^{2} \pi}{\lambda \Delta R_{C}}\left(\tau_{B}+\frac{2}{3} \tau_{R}\right)
\end{aligned}
$$$$
\left\{1+\frac{1}{2}\left(\frac{{ }_{B}}{B_{0}}\right)^{2}\left(\frac{{ }^{\top} Q}{T_{B}+2 / 3 \tau_{R}}\right)\right\}(M J / m)(4.2-9)
$$

where $\eta$ is the col: esistivity. For the RTPR. $B_{Q}=32.2 \mathrm{kG}, B_{0}=110 \mathrm{kG}, R_{c}=90 \mathrm{~cm}, \tau_{B}=0.08 \mathrm{sec}$, $T_{R}=0.02 \mathrm{sec}, \lambda=0.7, \Delta R_{s}=40 \mathrm{~cm}$, and $\eta_{\mathrm{Cu}}=1.7 \times 10^{6}$ ohm-cm (for $300 \mathrm{~K}$ copper). Hence. $\mathrm{W}_{\mathrm{T}}(\mathrm{Cu})=5.96 \mathrm{Mj} / \mathrm{m}$.

Eddy-Current Losses. Figure 4.2-1c shows schenaticallv the magnetic compression pulse of the reactor. having a linearly rising portion of duration $\tau_{R}$. For such a magnetic field the electrical skin depth is given by

$\delta_{\mathrm{ER}}^{2}=1 / 2 \times 10^{8} \pi \pi_{R}$.

The joule energy loss due to eddy currents induced in the coil during the rising and falling portions of the magnetic waveforms is shown to be'
$W_{E}(C: s)=\frac{2 \pi \bar{R}_{c} \prod_{0} B_{0}^{2}{ }^{T} R}{(0.4 \pi)^{2} \lambda \Delta R_{c}}\left(\frac{\lambda^{2} \Delta R_{c}^{2} d^{2}}{45.6 \delta_{E R}^{4}}\right)$

$(4.2-11)$

where $d$ is the coil layer thickness. Elininating $\eta$ between Egs. (4.2-10) and (4.2-11) yields

$$
W_{E}(\mathrm{Cu})=\frac{10^{-12} \bar{R}_{c} \mathrm{~B}_{0}^{2} \lambda \Delta \mathrm{R}_{c} d^{2}}{5.73 \delta^{2}}(\mathrm{MJ} / \mathrm{m})
$$

The total joule loss in the compression coil during the pulse is given by

$$
\begin{aligned}
& W_{J C}(C u)=W_{T}(C u) \\
& {\left[1+\left(\frac{T_{B}}{T_{B}+2 / 3 T_{R}}\right)\left(\frac{\lambda^{2} \Delta B_{C}^{2} d^{2}}{45.6 \delta_{E R}^{4}}\right)\right](M J / m)}
\end{aligned}
$$

The condition that the eddy current loss be only $3 \%$ of the transport current loss $\left(\mathrm{W}_{\mathrm{E}}(\mathrm{Cu}) / \mathrm{W}_{\mathrm{T}}(\mathrm{Cu})=0.03\right)$ yields the following relationship for the coil layer thickness.

$$
\mathrm{d}^{2}=\frac{1.36 \delta_{\mathrm{ER}}^{4}}{\lambda^{2} \Delta \mathrm{R}_{\mathrm{C}}^{2}}\left(\frac{\tau_{\mathrm{B}}+2 / 3 \tau_{\mathrm{R}}}{\tau_{\mathrm{R}}}\right)
$$

Setting $\tau_{R}=0.020 \mathrm{sec}, \delta_{\mathrm{ER}}$ equals $1.30 \mathrm{~cm}$ from Eq $(4.2-10)$. For $\tau_{b}=0.080 \mathrm{sec}$, the coil winding thickness is obtained from Eq. $(4.2-14)$ as $d \leqslant 1.5 \mathrm{~mm}$. Therefore, the compression coil is represented by a 187-turn transmission line of $\sim 0.5 \mathrm{~m}$ in length. For this design the eddy-current loss in the compression coil is $0.18 \mathrm{MJ} / \mathrm{m}$.

4.2.3.2 Radiation Heating in the Compression Coil. The neutronic calculations presented in Sec. 4.5.2 indicate 
that the neutron heating in the coil amounts to $0.57 \mathrm{MJ} / \mathrm{m}$ and the gamma-ray heating is $4.88 \mathrm{MJ} / \mathrm{m}$. Whence, including joule losses, a total of $11.6 \mathrm{MJ} / \mathrm{m}$ pulse is expected to be generated in the compression coil. This energy is not recoverable by the thermal conversion cycle and is sufficient to raise the temperature of the copper by $\sim \mathbf{2}$ /pulse, assuming uniform deposition and no heat loss. For a 10-sec power cycle $2.2 \mathrm{MW} / \mathrm{m}$ must be removed from the compression coil in the form of low$0^{-}$-d de heat. The significance of radiation damage in the compression coil materials is treated in detail in Sec. 5 .

4.2.3.3 Mechanical Stresses in the Compression Coil. The 110-kG adiabatic compression field will exert a pulsed outward pressure of $43 \mathrm{MPa}(6.3 \mathrm{kpsi})$ on the compression coil. This pressure could presumably approach that of a square-wave impulse resulting in an amplification factor of 2. The copper and titanium backup ring would then operate at a combined pulsed stress of approximately $260 \mathrm{MPa}$ (38 kpsi). Although this applied stress is higher than the $186 \mathrm{MPa}$ ( $27 \mathrm{kpsi}$ ) completely reversed fatigue strength at $10^{7}$ cycles for zirconium-copper alloy, the unidirectional nature of the loading will greatly increase this allowable limit. Titanium, which has a higher fatigue strength than copper, should present no problems. Obviously, more analytical investigations of the pulsed operating regime must be carried out. The unidirectional fatigue properties of zirconium-copper alloy will also have to be determined before the final geometry is selected. This basic design should not present serious structural problems within the copper if care is taken to avoid flux concentrations at end regions of the coil. However, the possibility of brittle fracture of intervening layers of ceramic or glass insulation must be considered in the overall coil design.

4.2.3.4 Mechanical Design. The compression coil is made of a high-strength, high electrical conductivity alloy (less than $1 \%$ zirconium) wound into a 187 -turn coil. Alternate insulation layers in the coil are slotted to allow the coolant to flow longitudinally through the coil. The coil operates near room temperature, although the gamma and neutron doses will be severe. Hence, electrical insulation must be inorganic and probably in a fabric form. A proposed coil assembly is shown in Fig. 4.2-2. When the coil is energized to its maximum field $(110 \mathrm{k}(\mathrm{i})$, the magnetic forces generate an effective pressure of approximately $43 \mathrm{MPa}$ (6.3 kpsi) acting radially ourward on the coil. To restrain this force, the coil is encased in a shell which furnishes azimuthal strength to the entire coil and blanket assembly; a high-strength titanium alloy has been tenatively selected because of its good fatigue strength.

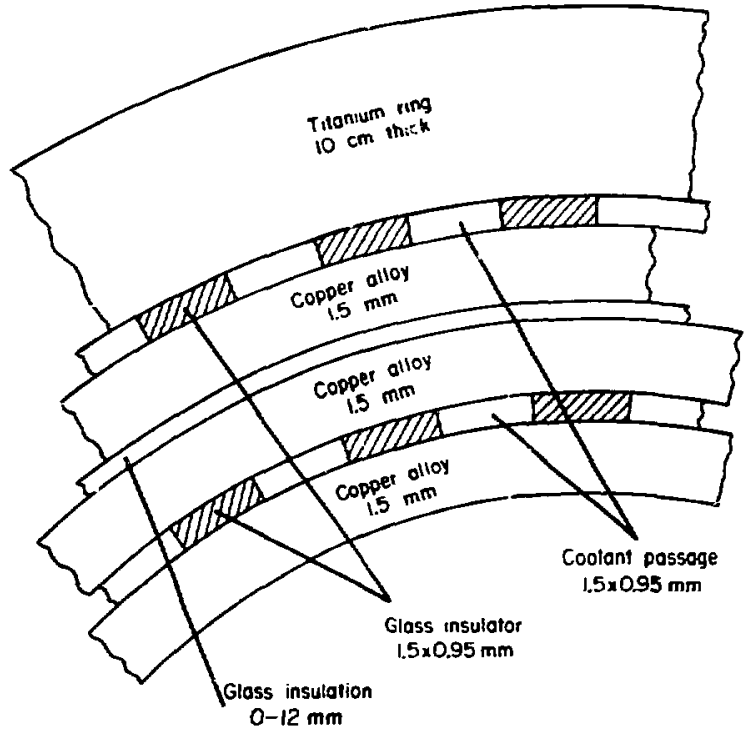

Fig. 4.2-2.

Schematic diagram of compression coil windings, insulation, and c. Jlant ducts.

Because of the radiation field, the coolant for the compression coil must be radiation-resistant, while maintaining a high dielectric constant. Since the cooling requirements are severe, inert gas may not be used.

Coil Structure. The structural requirements of the compression coil are quite severe. The internal pressure of $43 \mathrm{MPa}(6.3 \mathrm{kpsi})$ is suddenly imposed and released every cycle. If the coil itself furnishes no strength in hoop tension, the tension in the outer restraining ring exceeds $5.2 \times 10^{7} \mathrm{~N}$ (7000 tons) for the $0.5 \mathrm{~m}$ long restraining ring. If the copper coil is weak enough to act as a fluid which transmits the radial forces to the titanium ring, the radial strain may be severe enough to lead to fatigue failure within the copper coil. If this situation is proved by future analyses. a means must be found to internally strengthen the copper coil so that it can assume some portion of the load. The titaniurn outer shell provides a massive ring for mounting, moving, and positioning the segments.

Cooling. To minimize joule and eddy-current losses in the compression coil it must be kept as cool as possible. Since the heat load to the coil is not continuous but occurs primarily during the burn time of the cycle, a 
novel cooling concept was adopted. Nuclear radiation leaking through the blanket is the major contribution to the heat load: $0.57 \mathrm{MJ} / \mathrm{m}$ in neution heating and $4.88 \mathrm{M} ! / \mathrm{m}$ in gamma heating during the burn time. Ir. addition, $6.14 \mathrm{MJ} / \mathrm{m}$ of transport and eddy current joule losses within the coils are generated during the burn and subsequent plasma cooling. The latter loss is uniformly distributed throughout the coil, but the nuclear radiation energy deposition is, of course, more intense at tije inner portions of the coil (see Figs. 4.5-7 and 4.5-8). Because the burn time is so short $(\sim 70 \mathrm{msec})$, the amount of cooling which can in accomplished during that time is very small. As a result, the copper windings absorb that energy with an average temperature rise of about $2 \mathrm{~K}$. This temperature rise has little effect upon the operation of the windings during the burn time, but since the field is retained at $\sim 30 \%$ of its maximum jevel for about $1 \mathrm{sec}$ after the burning is quenched, the power losses during the plasma cooling portion of the cycle are increased as a result of the increased electrical resistivity of the wind. ings. These heat loads are imposed during eviry pulse.

Initially, cooling of the compression coil from t'te edge was studied. For a 1-m-long coil cooled from the ends, it was found that the teniperature rise at the axial center of the coil was $\sim 1300 \mathrm{~K}$, independent of the cooling effectiveness of the ends. Unless the coil length can be reduced below $\sim 1 \mathrm{~cm}$, edge cooling must be abandoned. As a result, the coil is cooled internally, as indicated in Fig. 4.2-2. Each turn of the compression coil is convectively cooled at one side.

The coolant has not yet been selected. In all likelihood, a radiation-resistant liquid with high dielectric strength will be selected. Since the compression coil will operate at low temperatures $(\sim 300 \mathrm{~K})$, organic coolants may represent a possibility; radiolytic decomposition can occur as long as deposition of resulting chars do not occur within the cumpression coil itself. Water is an ideal cooling medium, although the proximity to the lithium coolant discourages this choice. Chlorinated phenyls, transformer oils, or castor oil are satisfactory with respect to dielectric properties, although their room temperature stability in moderate radiation fields must be resolved. Gaseous coolants have been considered, but the high velocities $(20 \mathrm{~m} / \mathrm{sec})$ and high static prcssure $\left(7 \times 10^{5} \mathrm{~Pa}\right.$ ( 100 psi) for He) required are unatractive. Whatever coolant is selecied, however, it will flow conrinuously through the windings.

Because of the reat mass of copper in the coil, the heat transfer analysis can be treated with a bulk capacity approximation. During a burn pulse, the copper temperaturc rises only about $2 \mathrm{~K}$. This temperature rise does not represent an excessive perturbation of the steady state during which the temperature difference between coolant and copper will average about $5 \mathrm{~K}$. The important part of the cooling design, however, is to make certain that the coolant stay-time in the coil is less than sbout one-half the cycle time. Otherwise, the coolant temperature rise becomes excessive and retards the heat transfer. For this reason the coolant makes only a single pass through the 1/2-m length of the coil. Pertinent coolant and heat transfer parameters are shown in Table 4.2-1.

TABLE 4.2-1

\section{PARAMETER COMPRESSION COIL COOLING PARAMETERS}

Neutrun heating

Ganıma heating

Transport current joule heating

Eddy-current joule heating

Length of compression coil

I.D. of compression coil

O.D of compression coil

Each winding thickness (copper)

Number of turns

Insulation thickness (glass or ceramic)

Mean coil temperature

Coolant inlet temperature

Mean heat transfer coefficient

assumed for cooling

Average coil temperature rise per pulse

Coolant outlet temperature

Weight of copper

Weight of coolant (oil)
$0.75 \mathrm{MJ} / \mathrm{m} /$ pulse

$6.42 \mathrm{MJ} / \mathrm{m} /$ pulse

$5.96 \mathrm{MJ} / \mathrm{m} /$ pulse

$0.18 \mathrm{MJ} / \mathrm{m} /$ pulse

$0.5 \mathrm{~m}$

$1.87 \mathrm{~m}$

$2.67 \mathrm{~m}$

$1.5 \mathrm{~mm}$

187

$0.25 \mathrm{~mm}$

$298 \mathrm{~K}$

$293 \mathrm{~K}$

$44 \mathrm{w} / \mathrm{cm}^{2} / \mathrm{K}$

$2 \mathrm{~K}$

$296 \mathrm{~K}$

$17,100 \mathrm{kG} / \mathrm{m}$

$290 \mathrm{kG} / \mathrm{m}$

\section{REFERENCES}

1. S. C. Burnett, W. R. Eilis, T. A. Oliphant, Jr., F. L. Ribe. “A Reference Theta Pinch Reactor (RTPR)," Los Alamos Scientific Laboratory Report LA-51 21-MS (December 1972).

2. F. L. Ribe, S. C. Burnetr, W. R. Ellis, "Pulsed High-Beta Fusion Reactor," U.S. Patent 3,748,226, July 24. 1973.

\subsection{MAGNETIC ENERGY STORAGE AND TRANSFER (METS) SYSTEM*}

\subsubsection{System Requirements}

The function of the METS system is to store and return the large amount of magnetic energy $(378 \mathrm{MJ} / \mathrm{m})$ used in the adiabaric cumpression coil system. The magnetic

'J. Marshall, K. T. Thomassen, LASL. 
energy must be provided every cycle as a pulse having $\sim 30$-msec rise and fall time, with an $\sim 70$-msec flat top. Because of the large amount of energy transferrec to the compression field, losses in the METS system must be kept well below the electrical energy produced per pulse by the RTPR $(\sim 41 \mathrm{MJ} / \mathrm{m})$. Therefore, it is assumed that these losses, including refrigeration requirements, can be held below $2 \%(\sim 5 \mathrm{MJ} / \mathrm{m}$ per pulse) of the transferred energy; joule losses in the compressicn coil are not included in this loss criterion (Sec. 2.4).

\subsubsection{Principles of Operation}

A circuit to accomplish pulsed energy transfer in a reversible manner is shown in Fig. 4.3-1. Large amounts of magnetic energy $(286 \mathrm{MJ} / \mathrm{m})$ are stored in the cryogenic storage system, which in this example consists of modules containing three nested spherical coils and a transformer.

The two inner spherical coils of a module (each of which produces a uniform field in its interior and an

(a)

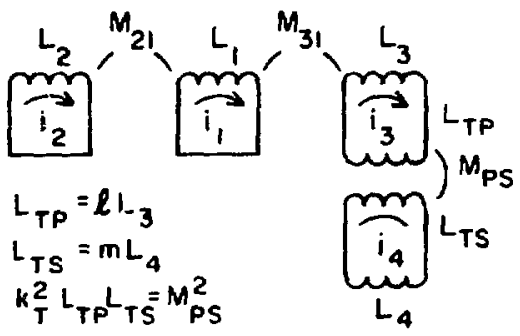

(b)

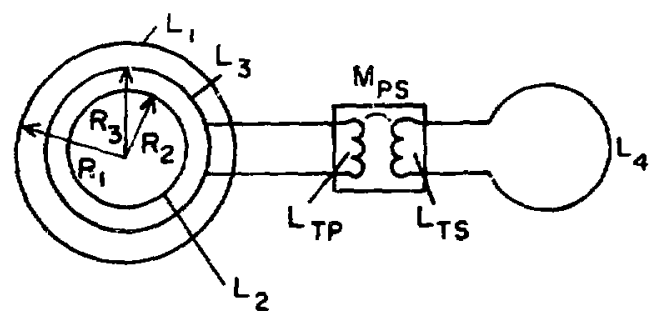

Fig. 4.3-I.

An chergy storage and transfer scbeme for the adiabatic compression scheme.

(a) An equivalent circuit representation.

(b) Schematic of nested coils:

$t_{i}=$ self inductance of $i^{t b}$ coil,

$M_{i j}=$ mut ual inductance.

Coil 4 represents the compression coil. exterior dipole field) have their magnetir fields at right angles, i.e., the coils are in quadrature or uncoupled. This pair of coils rotates inside an outer coil, alternately aligning the outer coil with one or the other of the inner coils. By rotating the inner coils from $\theta=-90^{\circ}$ to $\theta=+90^{\circ}$, the currents in the four circuits shown in Fig. 4.3-1 are induced as shown in Fig. 4.3-2. The transformer allows twice the current swing in the load, and the load current varies as

$\frac{1+\sin \theta}{2}$

or

$\int \frac{\omega(t) d t}{2}$

The distribution of the energy between the compression coil, transformer, and regions of the nested spherical coils is shown in Fig. 4.3-3 for three principal positions.

The coupling of the two inner coils to the outer coil varies, respectively, as $\sin \theta$ and $\cos \theta$, and, if the radii of the two inner coils are properly chosen, the total system energy is independent of the position of the inner coils. Thus, the rotor in theory can be turned with zero torque. This torque-free system was first proposed by P. F. Smith $^{1}$ of the Rutherford Laboratory, and further developed by Smith and Lewin ${ }^{2}$ and Thomassen. ${ }^{3}$

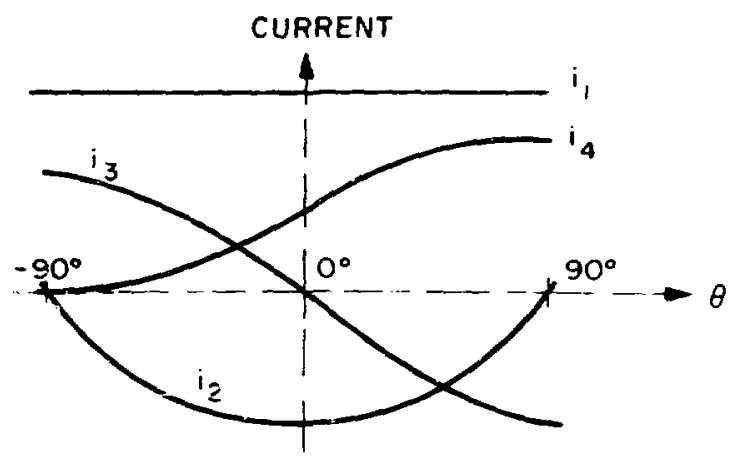

Fig. 4.3-2.

Variation of currents in the METS system as a function of rotor position. Coils 2 and 3 are mecbanically locked with tbeir magnetic axes in auadrature. The relative motion occurs between the $2-3$ thir and coil $t$. The current $i_{4}$ represents the current within the compression coil. 
(a)

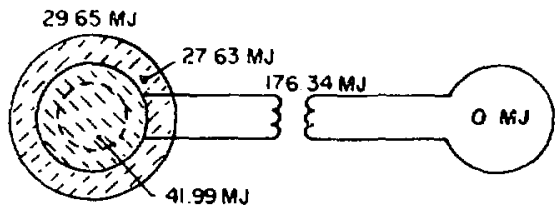

(b)

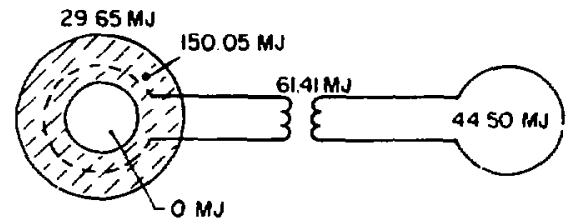

(c)

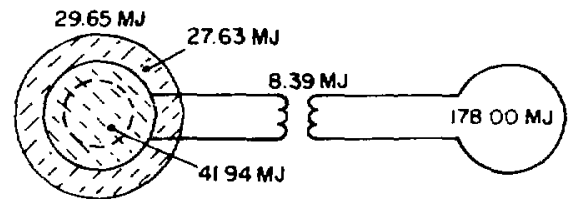

Fig. 4.3-3.

Distribution of stored energy for (a) $\theta=-90^{\circ},(b)$ $\theta=0^{\circ},(c) \theta=90^{\circ}$.

An artist's concept of the incorporation of a magnetic energy storage and transfer system into a theta-pinch reactor is shown in Fig. 4.3-4.

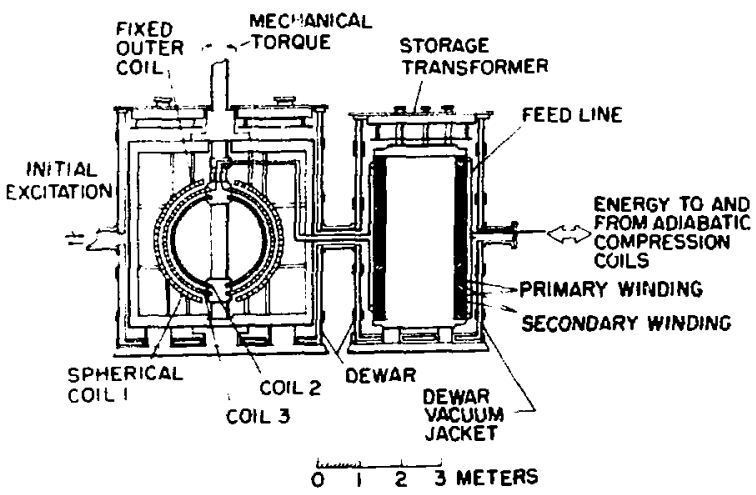

Fig. 4.3-4.

Artist's concept of METS system showing incorporation into a the ta-pinch reactor.

\subsubsection{Circuit Analysis}

Analysis of the circuit for the torque-free system proceeds as follows. Referring to Fig. 4.3-1 for current and inductance notation, the constancy of the flux linkages of the circuits in Fig. 4.3-1 gives the equations

$$
\begin{aligned}
\mathrm{L}_{1} i_{1}+M_{21} i_{2}+M_{31} i_{3} & =\text { const } \\
L_{2} i_{2}+M_{21} i_{1} & =\text { const } \\
(1+l) L_{3} i_{3}+M_{31} I_{1}+M_{T S} i_{4} & =\text { const } \\
(1+m) L_{4} I_{4}+M_{T^{2}} i_{3} & =\text { const }
\end{aligned}
$$

In normalized form, with $x_{j}=\sqrt{L_{j} j_{j}}, \beta_{T}=k_{T} \sqrt{\ell m}$, and $k_{i j}=M_{i j} / \sqrt{L_{i} L_{j}}$,

$$
\begin{aligned}
& x_{1}+k_{21} x_{2}+k_{31} x_{3}=y_{1} \\
& x_{2}+k_{21} x_{1}=y_{2} \\
& (1+\ell) x_{3}+k_{31} x_{1}+\beta_{T}=y_{3} \\
& k_{T} x_{3}+(1+m) x_{4}=y_{4}
\end{aligned}
$$

where $y_{i}$ represents explicit statements of the constants in Eqs. (4.3-1).

If the METS system is energized with coils 1 and 2 uncoupled, the net flux linkage is zero, i.e., $y_{2}=0$. Solutions to Eqs. 4.3-2 are then

$$
\begin{aligned}
& \mathrm{kx}_{1}=\mathrm{y}_{1} \\
& \mathrm{x}_{2}=-\mathrm{k}_{21} \mathrm{x}_{1} \\
& \mathrm{x}_{3}=-\alpha_{\mathrm{T}} \mathrm{k}_{31} \mathrm{x}_{1} \\
& \mathrm{x}_{4}=\mathrm{b}_{\mathrm{T}}\left(\mathrm{k}_{\mathrm{m}}+\mathrm{k}_{31}\right) \mathrm{x}_{1}
\end{aligned}
$$

where

$$
b_{T}=\frac{p_{T}^{2}}{1+l+m+l m\left(1-k_{T}^{2}\right)}
$$




$$
\begin{aligned}
\alpha_{T} & =\frac{1+b_{T}}{1+l}<1 \\
k & =1-k_{21}^{2}-\alpha_{T} k_{31}^{2} \\
k_{21} & \equiv k_{0} \cos b \\
k_{31} & \equiv k_{m} \sin \theta
\end{aligned}
$$

The $\theta$ variation depicted for $k_{21}$ in Eqs. (4.3-4) is a property of the spherical coils in quadrature.

To find the condition under which the energy is independent of angle, we calculate the total stored energy in the METS system:

$$
\begin{aligned}
W_{B O} & =\frac{1}{2}\left[x_{1}^{2}+x_{2}^{2}+x_{3}^{2}(1+l)+x_{4}^{2}(1+m)\right] \\
& +k_{21} x_{1} x_{2}+k_{31} x_{1} x_{3}+k_{T} x_{3} x_{4}= \\
& \frac{1}{2}\left(k+\alpha_{T} b_{T} k_{m}^{2}\right) x_{1}^{2} .
\end{aligned}
$$

The above equation is independent of angle if the parameter $K$ is a constant. A constant $K$ is achieved if $k_{o}{ }^{2}$ equals $\alpha_{\mathrm{T}} k_{\mathrm{m}}^{2}$, which infers that the coil radii are related by:

$$
\left(\frac{R_{2}}{R_{3}}\right)^{3}=\left(\frac{k_{0}}{k_{m}}\right)^{2}=\alpha_{T}<1 \quad(4 \cdot 3-6)
$$

Then,

$$
W_{B O}=\frac{1}{2}\left(1-k_{0}^{2}+b_{T} k_{0}^{2}\right) x_{1}^{2} \cdot(4 \cdot 3-7)
$$

The ratio of energy stored in the adiabacic compression coil, $W_{B}$, to that stored in the METS system, $W_{B O}$, is given by

$$
\begin{aligned}
\frac{W_{B}}{W_{B O}}= & {\left[2 b_{T} k_{m}\right]^{2} /\left[B_{T}^{2}-k_{m}^{2} b_{T}\left(1-b_{T}\right)\right.} \\
& (1+m)]
\end{aligned}
$$

The ratio $\mathrm{W}_{\mathrm{B}} / \mathrm{W}_{\mathrm{BO}}$ is maximized when $\ell$ is related to $m$ by the expression,

$\ell=\frac{1+m}{1+m\left(1-k_{T}^{2}\right)}\left[1-k_{m}+\frac{m_{m}^{2} k_{T}^{2}}{1+m}\right]$

This fraction of transferred energy is further maximized for the particular value of $m=\hat{m}$; if $W_{B} / W_{B O}$ equals $g(m)$, then $g(\hat{m})$ represents a maximum.

\subsubsection{Design Features}

Design parameters for the system are determined by first taking values for the coupling coefficients, $k_{T}$ and $k_{m}$, as large as practical. As reasonable maxima $k^{2}{ }_{T}$ and $k^{2} m$, respectively, are set equal to 0.98 and 0.90 . This situation requires good coupling in the transformer and implies that

$$
\frac{R_{3}}{R_{1}}=k_{m}^{2 / 3}=0.9655
$$

The maximum transfer fraction $W_{B} / W_{B O}$ is then 0.6232 and occurs for $m=1.380$ and $\ell=1.811$. These values of $\mathrm{m}$ and $\ell$ give $\alpha_{\mathrm{T}}=0.5612$ and $\mathrm{b}_{\mathrm{T}}=0.5775$. Therefore.

$$
\frac{R_{2}}{R_{1}}=\alpha_{T} k_{m}^{2}{ }^{1 / 3}=0.7964
$$

and $k_{0}^{2}=0.5051$.

If each METS unit supplies the energy for a meter of the reactor, the energy transferred to the load coil is 


$$
\left.\begin{array}{l}
W_{B}=\frac{B_{0}^{2}}{8 \pi} \times 10^{-5} \pi R_{i}^{2} \\
\left.1+\frac{1}{6}\left(\frac{R_{0}^{2}}{R_{i}^{2}}+2 \frac{R_{0}}{R_{i}}-3\right)\right]
\end{array}\right]
$$

where $\mathrm{B}_{\mathrm{O}}$ is the magnetic field used for adiabatic compression ( $k G$ ) and $R$ is in $\mathrm{cm}$.

With $B_{O}=110 \mathrm{kG}, R_{i}$ (coil inside radius) $=94.9 \mathrm{~cm}$. and $R_{o}$ (coil outside radius $=134.9 \mathrm{~cm}, W_{B}$ equals 178 $\mathrm{MJ} / \mathrm{m}$. Hence, $W_{\mathrm{BO}}=\mathrm{W}_{\mathrm{B}} / 0.6232=286 \mathrm{MJ} / \mathrm{m}$ and the energy stored in an isolated outer coil of the MFTS system, $W_{\mathrm{I}}=\mathrm{W}_{\mathrm{BO}} /\left(1-\mathrm{k}_{0}^{2}+\mathrm{b}_{\mathrm{T}} \mathrm{K}_{\mathrm{o}}^{2}\right)=364 \mathrm{MJ} / \mathrm{m}$.

Following current practice for large superconducting magnets, ${ }^{4}$ the uniform field inside the outer sphere if it were an isolated coil, $B_{01}$, is equated to $60 \mathrm{kG}$. The radius of this sphere is found from the relationship

$$
W_{1}=\frac{{ }_{01}^{2}}{8 \pi}\left(\frac{4}{3} \pi R_{1}^{3}\right)\left(10^{-7}\right)
$$

Therefore, $R_{1}=182 \mathrm{~cm}$, and from Eqs. (4.3-10) and $(4.3-11), R_{3}=176 \mathrm{~cm}$ and $R_{2}=145 \mathrm{~cm}$. An internal field $B$ is produced in a spherical coil of radius $R$ carrying a current per unit iength of $K_{\phi}(A / \mathrm{cm})$ equal to

\section{$\frac{3 B(k G)}{8 \pi} \times 10^{4} \sin \theta$}

Thus, the total ampere turns is given by

$$
\frac{3 R(\mathrm{~cm}) \mathrm{B}(\mathrm{kG})}{4 \pi} \times 10^{4}
$$

The maximum thickness and total quantity of the superconductor required are respectively and $\left.S(A-m)=75 \pi R^{2} i c m\right) B(k G)$ (note that $B_{3}=\alpha_{T} B_{01}$ ). Selection of the current density, J, then establishes the coil dimensions; $J$ is taken to be $10^{5} \mathrm{~A} / \mathrm{cm}^{2}$, essentially consistent with current practice 4 . The values for the dimensions and parameters are given in Table 4.3-1.

\subsubsection{Switching System}

No torque is required to effect the $180^{\circ}$ rotation of the mechanically linked inner pair of spherical coils insofar as the transfer of electrical energy is concerned. However, torque is required to overcome the mechanical in ertia of the coils. Since the coils are at rest at both the $-90^{\circ}$ and the $+90^{\circ}$ positions, there must be an initial angular acceleration followed by an angular deceleration. During the initial angular acceleration, the rotational ener$\mathrm{gy}$ of the coil pair must be increased from 0 to $1 / 2 \mathrm{I} \omega^{2}$; during the final angular deceleration this energy decreases to zero again. Except for frictional losses, therefore, the net mechanical energy transferred to the coil is zero, and the net mechanical energy required for the $-90^{\circ}$ to $+90^{\circ}$ switching rotation is only that required ro overcome frictional losses. The same process occurs in reverse during the reverse switching operation in which the coil pair rotates either backward $\left(+90^{\circ}\right.$ to $\left.-90^{\circ}\right)$ or forward $\left(90^{\circ}\right.$ to $270^{\circ}$ ).

The mechanical energy that must be supplied to overcome inertia is $1 / 21 \omega^{2}$. The moment of inertia of a spherical coil of thickness $\delta$, radius $R$ and density $\rho$ is $l_{\omega} \cong(5 / 8) \pi^{2} R^{4} \delta \rho$. With $\rho=5 \mathrm{gm} / \mathrm{cm}^{3}, I_{\omega_{3}}=1185$ $\mathrm{kG} \mathrm{m} \mathrm{m}^{2}$, and $\mathrm{l}_{\omega_{2}}=978 \mathrm{kG} \mathrm{m}$. Estimating the structural requirement by assuming hoop stress of $10^{5}$ psi indicates that the moments of inertia of coils 2 and 3 should be increased by factors of about 5 and 9, respectively. The moment of inertia of the rotor is then $15,560 \mathrm{kG} \mathrm{m}^{2}$.

Defining the risetine of the adiabatic compression field, ${ }^{T_{R}}$, by

$$
\left(\frac{d I}{d t}\right)_{\max }=\frac{I_{\max }}{T_{R}}
$$

and since the current in the compression coil is described by $(1+\sin \theta) / 2$. I*or a constant angular velocity the transfer oscurs is a time $(\pi / 2) \tau_{R}$. $\frac{3 B(k G) \times 10^{4}}{8 \pi J\left(A / \mathrm{cm}^{2}\right)} \quad(\mathrm{cm})$ 
TABLE 4.3-1

TR PARAMETERS FOR A SUPERCONDUCTING ENERGY TRANSFER MODULE

\begin{tabular}{l} 
Symbol \\
\hline$k_{T}^{2}$ \\
$k_{m}^{2}$ \\
$W_{B} N_{B O}$ \\
$B_{01}$ \\
$W_{B O}$ \\
$W_{B}$ \\
$R_{1}$ \\
$R_{2}$ \\
$R_{3}$ \\
$(N)_{1}$ \\
$(N)_{2}$ \\
$(N)_{3}$ \\
$J$ \\
$\delta_{1}$ \\
$\delta_{2}$ \\
$\delta_{3}$ \\
$V_{1}$ \\
$V_{2}$ \\
$V_{3}$ \\
$I_{W 1}$ \\
$I_{W 2}$ \\
$I_{W 3}$
\end{tabular}

Definition
Coupling coefficient
Coupling coefficient
Transfer efficiency
Field strength of coil 1
Total magnetic energy stored
Energy transferred to load
Outer coil radius
Inner coil radius
Intermediate coil radius
Ampere turns for outer coil
Ampere curns for inner coi]
Ampere turns for intermediate coil
Current density in superconductor
Inner coil thickness
Outer coil thickness
Intermediate coil thickness
Superconductor volume for inner coil
Superconductor volume for outer coil
Supercusuctor volume for
intermediate coil
Moment of inertia of superconductor
winding of inner coil
Moment of inertia of superconductor
winding of outer coil
Moment of inertia of superconductor
winding of intermediate coil

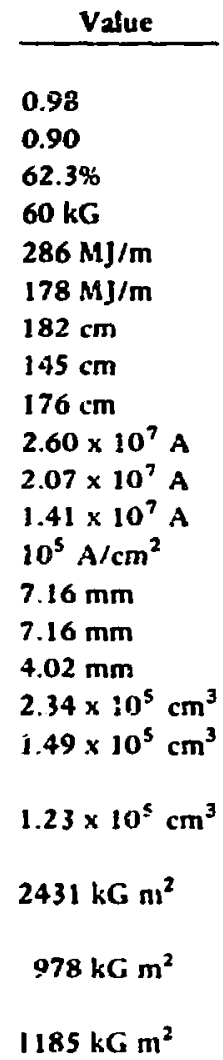

For $\tau_{R}=20 \mathrm{msec}$ the rotational or inertial energy becomes $77.8 \mathrm{MJ} / \mathrm{m}$. which is an appreciable fraction of the stored energy $(286 \mathrm{MJ} / \mathrm{m})$. Since the inertial energy scales as $\left(W_{B O}\right)^{4 / 3} /\left(\mathrm{J} \mathrm{B}_{01}{ }^{5 B} T_{R}^{2}\right)$, increasing the risctime $\tau_{R}$ presents an obvious method for reducing the large incrtial energy. $W_{B O}$ is also an optimizable parameter. The develapment of superconductors with higher critical field-current products will decrease the inertial energy. (These developments should also improve the cost since the superconductor volume scales like $B_{01}^{-1 / 3} T^{\prime}$ ). Also, the use of more modules with iess energy per unit cuts down the fractional energy in incrtia, since this fraction scales as $W^{1 / s}$. Although the incrtial energy requirement of $77.8 \mathrm{MJ} / \mathrm{m}$ is unaccepeable and methods for reducing this value are both obvious and promising, the MIETs design described hercin will keep the value of $77.8 \mathrm{MJ} / \mathrm{m}$. Obviously. futuze work will be needed in this area.

To provide the $77.8 \mathrm{MJ} / \mathrm{m}$ of inertial energy requires a mechanical torquing system with its attendant losses; these lesses represent the principal drawback to this scheme. Should $100 \mathrm{kG}$ superconductors (at $10^{5} \mathrm{~A} / \mathrm{cm}^{2}$ ) become available, the inertial energy would be reduced to $33.2 \mathrm{~mJ} / \mathrm{m}$ or $12 \%$ of the total stored energy. For this case the torque can be derived internally by saturable core control inductors in series with the load. The introduction of a variable inductance makes $W_{B O}$ a function of $\theta$, and internal torque is developed. ${ }^{2}$

$\Lambda$ better method for supplying the inertial energy is to make the radius of the innermost coil $\left(R_{2}\right)$ smalter than the value required to make the energy constant. In this 
case, the energy depends on the rotor position and is minimum at $\theta=0$. The energy difference between the initial and intermediate positions is available to do work. the torque being $-\mathrm{d} W / \mathrm{d} \theta$. By reducing the inner radius by $\sim 1 \%$, the rotor can be rotated from $-80^{\circ}$ to $+80^{\circ}$ in 20 msec with $10 \%$ of the initial energy transferred to kinetic energy at the "bottom of the potential well" in the intermediate state $(\theta=0)$.

To supply the required inertial energy, $k_{0}^{2}$ is made smaller than the value given by Eq. $(4.3-6)$ by an amount $\epsilon^{2}$

$$
k_{0}^{2}=\alpha_{T} k_{m}^{2}-\epsilon^{2}
$$

For this case Eq. (4.3-5) becomes

$$
\begin{gathered}
W_{B O}=\frac{1}{2}\left[1-\alpha_{T} k_{m}^{2}+\alpha_{T} b_{T} k^{2}\right. \\
\left.+\varepsilon^{2} \cos ^{2} \leftarrow\right] x_{1}^{2}
\end{gathered}
$$

This system produces no external electrical input, so the flux $y_{1}=K x_{1}$ is constant, with

$$
k=1-k_{0}^{2} \cos ^{2} b-\alpha_{T} k_{m}^{2} \sin ^{2} b \text {. }
$$

Since the initial and final energies are unchanged from previous values, the same fraction of the total energy is transferred. Only the intermediate state in Fig. 4.3-3 is different; a portion of the magnetic energy now appears as inertial energy.

The torque determined with the aid of Eq. (4.3-16) is given by

$-\left(d W_{30} / d b\right)_{y_{1}}=\frac{1}{2}=\frac{1}{2}\left(1+\frac{2 \alpha_{T} b_{T^{2}} k^{2}}{K}\right)$

$\left(\frac{y_{1}}{k}\right)^{2} \frac{d k}{d t}=-\frac{1}{2} x_{1}^{2} \epsilon^{2} \sin 2 b\left(1+\frac{2 \alpha_{T}{ }^{b} T_{m}^{2}}{k}\right)$

$(4 \cdot 3-18)$

The time required to rotate from $\theta=-\theta_{0}$ to $\theta_{0}$ is found from the eriengy balance, $\frac{1}{2} I_{\omega} \ddot{\theta}^{2}=W_{B O}\left(\theta_{0}\right)-W_{B O}(b)$

Hence, the rotational time for $\theta$ to go from $-\theta_{0}$ to $\theta_{0}, \tau$. is given by

$\tau=\int_{0}^{\sigma_{0}} \sqrt{\frac{2 I \omega}{W_{B O}\left(\theta_{0}\right)-W_{B O}(\sigma)}} d \sigma$

For $\theta_{0}$ near $90^{\circ}$ and for small $\epsilon$,

$\tau=\left(\frac{1}{\varepsilon C}\right) \sqrt{I_{\omega} / W_{B O}\left(90^{\circ}\right)} \quad \ln \left(\frac{1+\sin \theta_{o}}{\cos \theta_{0}}\right)$

$(4.3-21)$

where

$$
c^{2}=\frac{1-\alpha_{T} k_{m}^{2}+2 \alpha_{T} b_{T} k_{m}^{2}}{\left(1-\alpha_{T} k_{m}^{2}\right)\left(1-\alpha_{T} k_{m}^{2}+\alpha_{T} b_{T} k_{m}^{2}\right)}
$$

For the design given originally, ${ }^{3}$ the time calculated from Eq. (4.3-21) with $\theta_{\alpha}=80^{\circ}, \mathrm{I}_{\omega}=839 \mathrm{kCi} \mathrm{m}^{2}$. and $\mathrm{W}_{\mathrm{BO}}\left(90^{\circ}\right)=225 \mathrm{MJ} / \mathrm{m}$, is

$T=\frac{4.01}{\epsilon} \mathrm{msec}$

To achieve a 20-msec time between start and fu!l con:pression field, $B_{0}$, requires $\epsilon=0.2$ so that $k_{0}^{2}$ is reduced by $4 \%$ and the inner radius by $1.33 \%$. Such a reduction transfers nearly $10 \%$ of the stored energy to rotational energy at the mid-state, $\theta=0$. For small $\epsilon$ the rotational energy varies as $\epsilon^{2}$, the time as $\epsilon^{-1}$, and the inner radius is reduced by $1-1 / 3 \epsilon^{2}$.

A rough cost estimate of the system assuming $\mathrm{Nb}_{3} \mathrm{Sn}$ wire can be made using costs given by Smith and Lewin. ${ }^{2}$ Wire costs in dollars are assumed to equal $10^{-4} \mathrm{~B}_{\mathrm{o}} \mathrm{S}$ where $B_{0}=$ field strength $(k G)$ and $S=$ quantity of superconductor in ampere-meters. For the spherical coils,

$$
S=\sum_{i} \frac{3}{2} \pi^{2} R_{i}^{2} H_{o i}
$$


and the sum is made over each coil. Using the quantities in the design, including the primary of the transformer, which is also superconducting, gives a wire cost of $\$ 3.7 \mathrm{M}$. Using a cost of 25 to $50 \mathrm{cents} / \mathrm{cm}^{2}$ of surface for the cryostat, 25 cents $/ \mathrm{cm}^{2}$ for refrigeration. and fabrication cost of 1.5 times wire costs $(55.5 \mathrm{M})$ gives a total of $59.5 \times 10^{6}$ for each $285 \mathrm{MJ}$ unit, or $: 5-1 / 2$ cent per joule.

\subsubsection{AC Losses and Heat Load}

Because the rotur is at rest most of the time, the ac losses are greatly reduced. Present superconducting mag. net designs reduce eddy current losses to values less than the specific superconducting hysteresis losses. An expression for these is

$$
W_{E} \sim \operatorname{BSd} / 2.10^{8} \text { (J per cycle) }
$$

For this case, $S$ is the quantity of superconductor expressed in $(A-\mathrm{cm})$ as given in Eq. $4.3-23, \mathrm{~J}(\mathrm{~cm})$ is the diameter of the superconductor filaments, and $B$ is in gauss. The ratio of heat dissipated to the energy stored is

$W_{E} / W_{B O} \sim d / R \sim 5 \times 10^{-6} \rightarrow 5 \times 10^{-5}$

since $d \sim 10^{-3}$ to $10^{-2} \mathrm{~cm}$ and $R \sim 200 \mathrm{~cm}$. Ideaily at $4 \mathrm{~K}$, the refrigeration losses are $\sim 1 \%$ of $\mathrm{W}_{\mathrm{BO}}$; the ac heat load is a minor factor.

To assume that eddy current losses can be kept small at the anticipated pulse times, however, inay be overly optimistic. This matter will depend on iuture developments in the technology of superconductiıg magnets.

The remaining heat load in the cryogenic system arises from the electrical connection to the room-temperature compression coil. It is anticipated that the connection will be a graduation of several superconducting cryogenjc fluids with the connection to the supersonductor being cryogenic aluminum, also at $4 \mathrm{~K}$. The refrigerator power for such a system has not yet been calculated.

\subsubsection{Concluding Ilemarks on the METS System}

The forcgoing analysis of the METS system has been made with an optimistic choice of design parameters, and undoubtedly much inore experience and information is required. The current density, $\mathrm{J}=10^{5} \mathrm{~A} / \mathrm{cm}^{2}$, may be high in that stnuctural materials and their attendant dilution of the current density have been omitted. Also, by today's standards for superconductors, the density of 5 $\mathrm{g} / \mathrm{cm}^{3}$ is low. Both factors reflect optimism in the results of the MFTS system analysis, and these results depend heavily upon long-term advances in the development of superconductors. The high inertial energy reported herein will be increased considerably by use of more realistic current densities $\left(5 \times 10^{4}\right.$ to $\left.2 \times 10^{4} \mathrm{~A} / \mathrm{cm}^{2}\right)$ as well as increased superconductor densities. On the other hand, th: BETS system inertial energies can be reduced by reasonable increases in the risetime, $\tau_{R}$; risetimes of the order of 100 msec will require reassessment of the system described in this report. and must await future analyses. The results of a parametric burn calculation and the effects of larger riserimes on the thermonuclear energy output are summarized in Sec. 2.2 (Fig. 2.2-3).

Future studies on the theta-pinch system will focus on other means of transferring reversibly the large quantity. of energy hetween a superconducting magnetic energy store and the adiabatic compression coil. For instance, a normal-going switch in conjunction with a transfer capacitance offers a possible alternative to the system previously descrihed. A schematic drawing of the alternative sy'stem is given in 1:ig. 4.3-5.

A transfer capacitor, connected across the current breaker of an inductive energy storage system, can in principle make the system non-dissipative. This is in contrast to the situation where the breaker is shunted with a resistor or with an electric arc generated when the switch is opened. In the resistive shunt case, $50 \%$ of the initia! stored energy is dissipated in the resistor, or the are, or both, and a maximum of $25 \%$ is transferred to the load, assuming the load to be purely inductive. If there were nc crowbar switch, $S_{3}$. the circuit would oscillate with energy transferring back and forth between $L_{1}$ and $L_{2}$. The oscillation frequency is given by

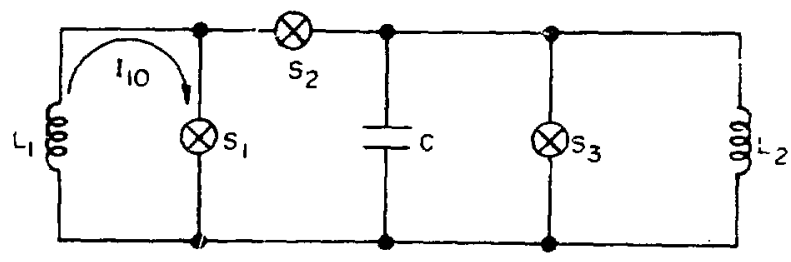

Fig. 4.3-5.

Transfer capacitor circuit. $L_{1}$ is the energy storage inductance. $s_{1}$ is the energy transfer current breaker, $C$ is the transfer capacitor, $S_{2}$ is the current breaker for charging $L_{1}, S_{3}$ is the load crowbs switcb, $L_{2}$ is the load inductunce, and $L_{1}=L_{2}$ represcnts the condition for full encrgy transfer. 
$\omega=\frac{1}{\sqrt{1 / 2 L_{1} \mathrm{C}}}$.

Transfer is initiated by opening $S_{1}$ ifter closing $S_{2}, S_{3}$. initially open, is ciosed one-half period after opening $S_{\mathbf{1}}$. At midpoint of the transfer time, the current in $L_{1}$ is one-half its initial value, the current in $L_{2}$ is one-half its final value, and so the energies are one-fourth of initial and final values. This adds up to one-half of initial or final energy. The remaining half is stored temporarily in the transfer capacitor. The currents and energies in the circuit are shown in Fig. 4.3-6 and 4.3-7 as functions of time.

In principle, a transfer capacitor can transfer energy from inductive storage to an inductive load with nearly $100 \%$ efficiency. The transfer capacitor must be able to store half the energy transferred, but does so on a pulse basis with no voltage reversal. A homopolar motor generator repiesents a strong candidate for the transfer capacitor indicated in Fig. 4.3-5. The mechanical and electrical design of a homopolar motor generator is outlined in Appendix D. For instance, with an axial magnetic field of $50 \mathrm{kG}$, a two-disk homopolar motor, with dimensions $1 \mathrm{~m}$ diameter by $5 \mathrm{~cm}$ thick represents a capacitance of $\sim 90 \mathrm{~F}$ and with a maximum voltage of $230 \mathrm{~V}$, will store $\sim 8 \mathrm{MJ}$ of energy. The small compact form of a homopolar motor and the large amount of energy storage makes this concept atractive. Future work on the METS system will seriously consider the homopolar motor as a transfer element or, perhaps, the main energy store.

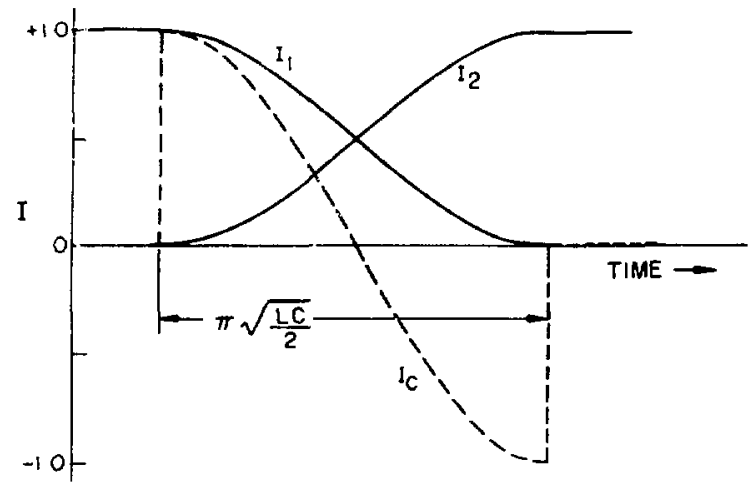

Fig. 4.3-6.

C.urrents in the circuit depicted in Fig. 4.3-5 as a finction of time, assuming $L_{1}=L_{2} . S_{1}$ is opened instantaneously and $S_{3}$ is closed at the maximum current in $L_{2}$

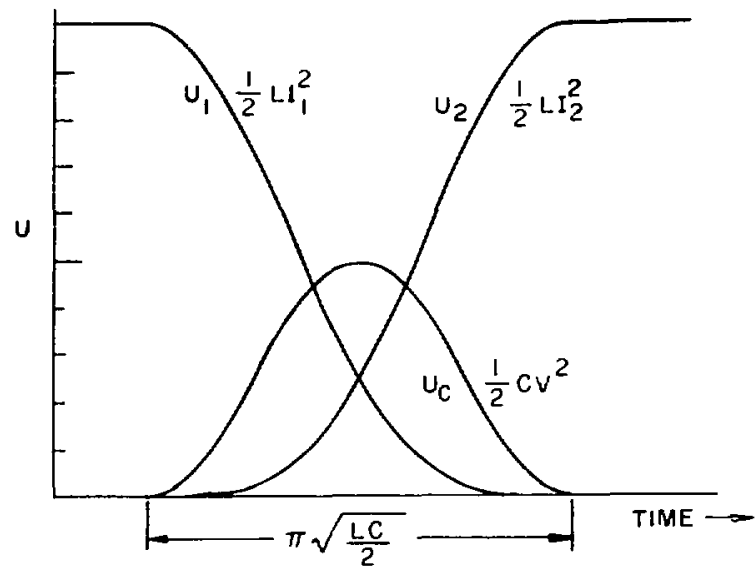

Fig. 4.3-7.

Energies us time for the condilions described in Figs. 4.3-5 and 4.3-6.

\section{REFERENCES}

1. P. F. Smith, Synchrotron Power Supplies Using SuperconJucting Electrical Storage, Proc. 2nd Int. Conf. on Magnet Technology, Oxford, 1967, p. 589.

2. P. F. Smith and J. D. Lewn, Particle Accelerators, Vol. 1, p. $155,1970$.

3. K. I. Thomassen, "Reversible Magnetic Energy Transfer and Storage Systems," Los Alamos Scientific Laboratory Report LA-5087-MS (November 1972), and paper presented at the Texas Symposium on the Technology of Controlled Thermonuclear Fusion Experiments and the Engineering Aspects of Fusion, University of Texas at Austin, Nov, 20-22, 1972.

4. J. Purcell, H. Desportes, and D. Jones, "Superconducting Magnet for the 15 Foot NAL Bubble Chamber," ANL/HEP 7215, February, 1973.

\subsection{FIRST-WALL SYSTEM*}

\subsubsection{System Requirements.}

The first-wall system is a bonded composite of insulator and metal; materials chosen for this system are alumina and niobium. The mechanical and structural functions of the first-wall system are (1) the maintenance of good vacuum integrity between the blanket and the plasma regions of the core ( $\sim 1 \mathrm{~m}$ Torr base pressure) and (2) the containment of the lithium coclant and pressure surges associated with the highly transient flow. The electrical function of the first-wall system must prevent azimuthal or radial shorting of the plasma Bond integrity

-R. A. Krakowski, LASL; J. Cook, T. A. Coultas, ANL. 
between the insulator and metal must be assured for reasons of heat transfer. Table 4.4-1 summarizes the thermal and radiation flux loadings under which the firstwall system is expected to operate. The major operational and environmental conditions under which the first wall must operate are:

- Cyclic temperature transients and the ensuing thermal stress will occur with a frequency of $1 / \tau_{c}(\sim 0.1 \mathrm{cps})$ or $2.6 \times 10^{6}$ transients per year for a plant operating factor of 0.85 .

- Nuclear transmutation of both insulator and metal will cause changes in modulus, strength, density, and electrical and thermal properties. Approximately 0.5 at. $\%$ of the aluminum is expected to be transmuted each year.
- Dimensional change will be induced by the high neutron fluences to which the first wall will be subjected ( $\sim 9 \times 10^{22}$ neutrons $/ \mathrm{cm}^{2}$ /year).

- Short-term changes in electrical resistivity and dielectric breakdown strength will occur during the burn portion of the power cycle; these property changes must be recover. ed before the beginning of the next power cycle.

The RTPR concept requires good electrical properties of the insulator only during the implosion heating stage of the power cycle; in the intense neutron and bremsstrahlung flux generated by the bum, the insulator has virtually no electrical requirement. Long-te' $m$ degradation of all first wall properties is expected, hcwever, and identification of long-te:m failure modes is equally as:

TABLE 4.4.1

\section{THERMAL AND RADIATION FLUX LOADING ON FIRST WALL"}

\section{Definition}

Uncollided (14.08 MeV) neutron current averaged over the reactor cycle $\left(\tau_{c}=10\right.$ seconds)

Uncollided (14.08 MeV) neutron current averaged over the burn time

Total neutron flux averaged over the reactor cycle

Fluence of $14.08 \mathrm{MeV}$ neutrons

Current of 14.08 neutrons/pulse

Neutron power density in $\mathrm{Al}_{2} \mathrm{O}_{3}$

Gamma ray power density in $\mathrm{Al}_{2} \mathrm{O}_{3}$

Neutron power density in $\mathrm{Nb}$

Gamma ray power density in $\mathrm{Nb}$

Bremsstrahlung energy flux at the first wall

Bremsstrahlung power density in $\mathrm{Al}_{2} \mathrm{O}_{3}$

Bremsstrahlung power density in $\mathrm{Nb}$

Total power density in $\mathrm{Al}_{2} \mathrm{O}_{3}$

Total power density in $\mathrm{Nb}$
Value

$8.91 \times 10^{13} \mathrm{n} / \mathrm{cm}^{2} \mathrm{sec}$

$1.10 \times 10^{16} \mathrm{n} / \mathrm{cm}^{2} \mathrm{sec}$

$5.13 \times 10^{14} \mathrm{n} / \mathrm{cm}^{2} \mathrm{sec}$

$2.84 \times 10^{21} \mathrm{n} / \mathrm{cm}^{2}$ year $8.91 \times 10^{14} \mathrm{n} / \mathrm{cm}^{2}$ puise

$156.3 \mathrm{~J} / \mathrm{cm}^{3}$ pulse

$63.5 \mathrm{~J} / \mathrm{cm}^{3}$ pulse

$23.6 \mathrm{~J} / \mathrm{cm}^{3}$ pulse

$183.4 \mathrm{~J} / \mathrm{cm}^{3}$ pulse

$71.62 \mathrm{~J} / \mathrm{cm}^{2}$ pulse

$1432.4 \mathrm{~J} / \mathrm{cm}^{3}$ pulse

$286.5 \mathrm{~J} / \mathrm{cm}^{3}$ pulse

$1652.2 \mathrm{~J} / \mathrm{cm}^{3}$ pulse

$493.5 \mathrm{~J} / \mathrm{cm}^{3}$ pulse

${ }^{a}\left(I_{W}=2.0 \mathrm{MW} / \mathrm{m}^{2}, \tau_{B}=.070 \mathrm{sec}, \tau_{c}=10 \mathrm{sec}, b=50 \mathrm{~cm}\right)$ 
important as the anticipation of short-term effects. This section summarizes the design requirements, operating conditions, and the effects of plasma cycle parameters on the first wall. The potential failure modes of the first wall system is treated in Sec. 5.

\subsubsection{The First-Wall Insulator}

A discussion of the properties of alternative insulators is given in Sec. 5. A desired property of the insulator is a high dielectric strength at elevated temperature. At the outset of the program it appeared that a value as high as $200 \mathrm{kV} / \mathrm{cm}$ could be attained by accounting for optimistic effects of a thin section, very short pulse time, retardation of electrical breakdown by the magnetic field, and a composite material between alumina and niobium. Subsequent experimental data ${ }^{\prime}$ indicate that at temperatures up to $1100 \mathrm{~K} 400 \mathrm{kV} / \mathrm{cm}$ can be obtained with polycrystalline alumina. The breakdown strength under transient conditions is generally known ${ }^{2}$ to exceed dc breakdown by a factor of $\sim 2$.

The insulator thickness, $x_{D}$, is determined from the following expression

$x_{D}=\frac{2 \pi b E_{\theta}}{2 N E_{D}}$

where $E_{D}$ is the design dielectric strength, $N$ is the number of segments around the plasma minor radius (see Fig. 3.2-1), and $E_{\theta}$ is the tangential electrical field. The required electric field was obtained by adjusting the ratio of implosion to compression heating. The number of segments, $N$, is chosen as a maximum consistent with tritium breeding (the radial metallic dividers required between segments increase neutron absorption without tritium breeding). Furthermore, $N$ must be large enough (greater than 25) to prevent excessive eddy current losses and MHD induced pressures in the lithium coolant channels (see Sec. 4.5-4). Employing a safery factor of 2 with respect to an assumed dielectric strength of $200 \mathrm{kV} / \mathrm{cr}_{1}$, a thickness of $0.031 \mathrm{~cm}$ of insulation was chosen for $\mathrm{F}_{\theta}=2 \mathrm{kV} / \mathrm{cm}$ and $\mathrm{N}=100$ segments. On the basis of new breakdown data ${ }^{1}$ on $\mathrm{Al}_{2} \mathrm{O}_{3}$ it appears that this insulator thickness can be reduced if necessary to relieve excessive thermal stresses.

Another fortunate result from those preliminary experiments on pulsed dielectric strength of alumina is the apparent constancy of dielectric strength with temperature rather than the precipitous drop of steady-state dielectric strength data as temperature increases (Fig. 5.1-1).
As mentioned in Chapter 2, both conventional and advanced power plants have been designed using, respectively, the inlet coolant temperatures of $683 \mathrm{~K}$ and $1273 \mathrm{~K}$. Anticipating a pos dielectric strength at high temperature, many calculations on the first wall and blanket portions of the power plant used a coolant inlet temperature of $68 \mathrm{~s} \mathrm{~K}$. It now appears that a coolant inlet temperature of $1273 \mathrm{~K}$ may not serious!y degrade the dielectric strength. Further ins ilator experimentarion, particularly with respect to radiation effects, is needed before definite conclusions on the first-wall temperature can be made.

In addition to good dielectric strength, the first-wall insulator must have good thermal properties and mechanical strength. The desired (design) thermal conductivity has been set ac $0.1 \mathrm{~W} / \mathrm{cm} \mathrm{K}$ and the heat capacity is $5.0 \mathrm{~J} / \mathrm{cm}^{3} \mathrm{~K}$. Thermal stress calculations discussed in Sec. 4.4.5 indicate that eyclic compressive strengths of the order of $3.3 \times 10^{8} \mathrm{~Pa}(\sim 50 \mathrm{kpsi})$ will be required.

The electrical and thermal requirements of the insulator are not separable. High dielectric strength and electrical resistivity will permit thinner insulator layers, thereby reducing temperature differences across the insulator. The reduced temperature differences result in lower thermal stresses and less chance for fracture of the insulator.

Although $\mathrm{Al}_{2} \mathrm{O}_{3}$ is considered a reference insulator material, the large amount of neutron-induced swelling in $\mathrm{Al}_{2} \mathrm{O}_{3}\left(1-10 \mu \mathrm{m}\right.$ grair size, $\sim 6$ vol. $\%$ per $10^{22}$ nvt, $\mathrm{E}_{\mathrm{N}}>0.1 \mathrm{MeV}^{3}$ presents a severe limitation. The electrical, thermal, and structural characteristics of $\mathrm{Al}_{2} \mathrm{O}_{3}$. however, are close to the aforementioned design values. Details of the anticipated material problems as well as alternative materials for the first-wail insulator are given in Sec. 5.1-1.

\subsubsection{The First-Wall Meta!}

Because of the high-temperature, liquid-metal environment to which the first wall metal will be exposed, the RTPR design (like most fusion reactor designs) uses niobium as the major construction material in the first wall and blanket. Whatever the first-wall metal, however, the atom displacement rate, helium generation, and transmutation will be great. Thesc problems as well as radiation induced swelling, fatigue failure, corrosion, and insulator bonding are treated in Sec. 5.1.2, and 5.4.

\subsubsection{Heat Travısfer at the First Wall}

A two-dimensional finite-clement heat transfer code has been programmed to study the thermal transient 


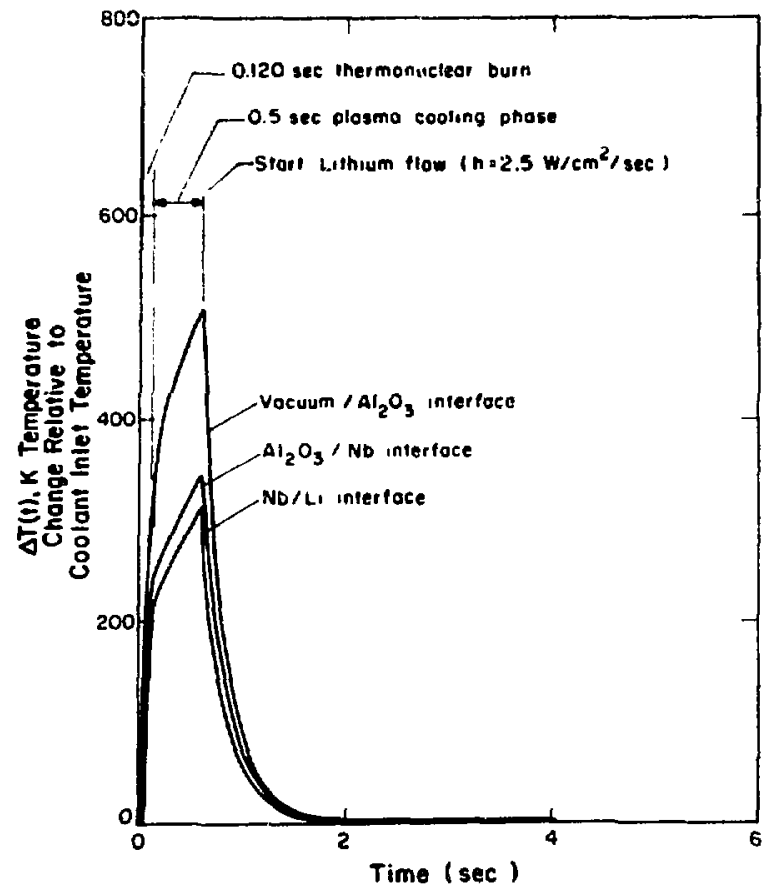

(A)

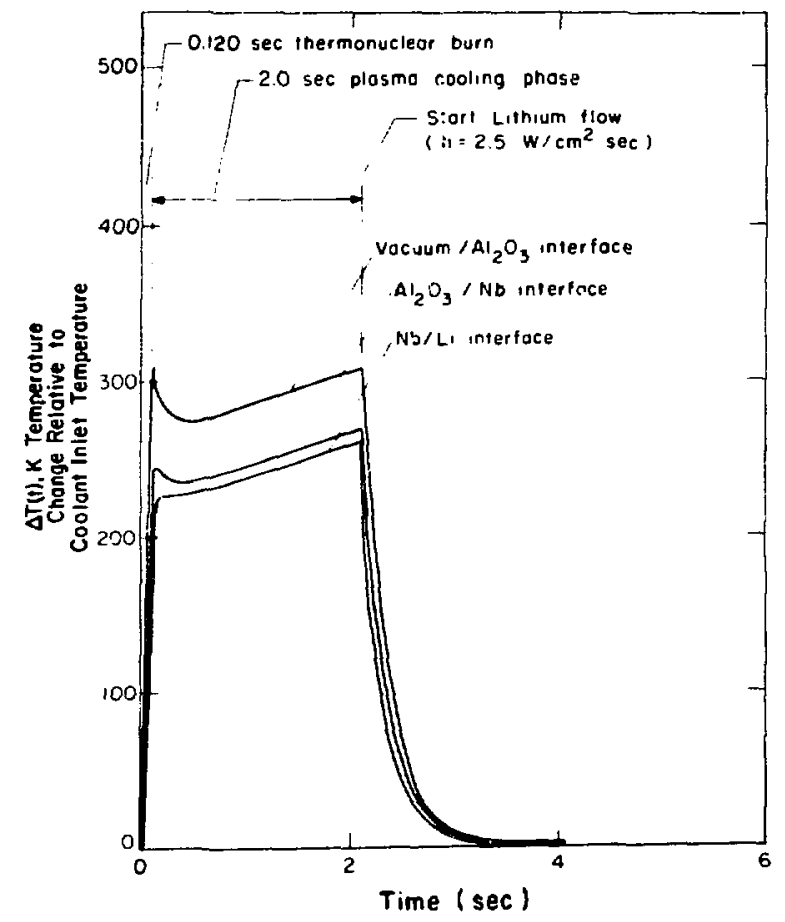

(C)

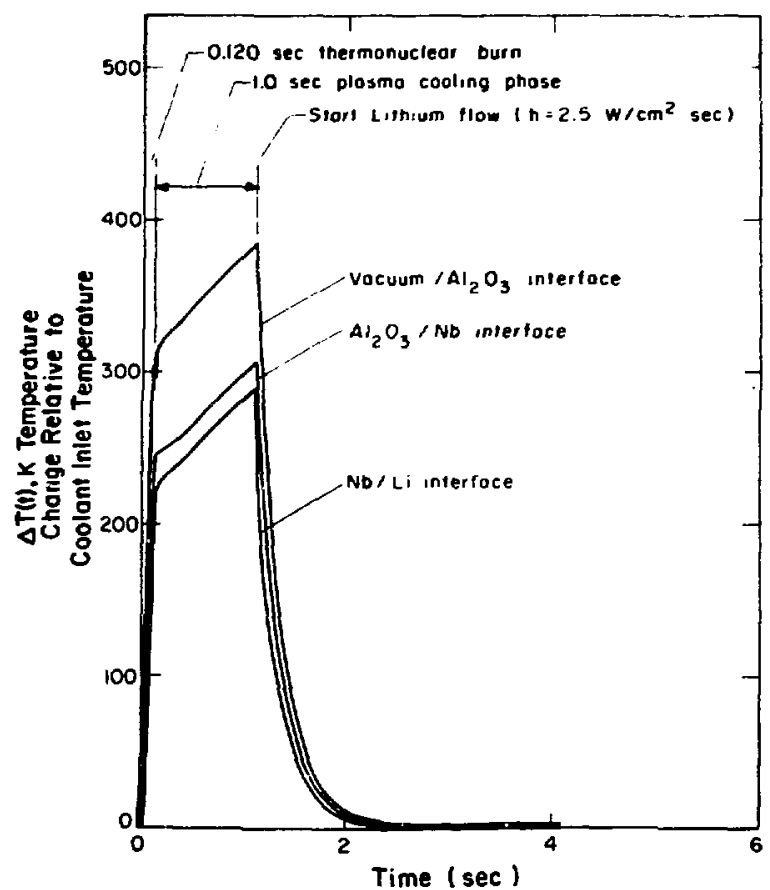

(B)

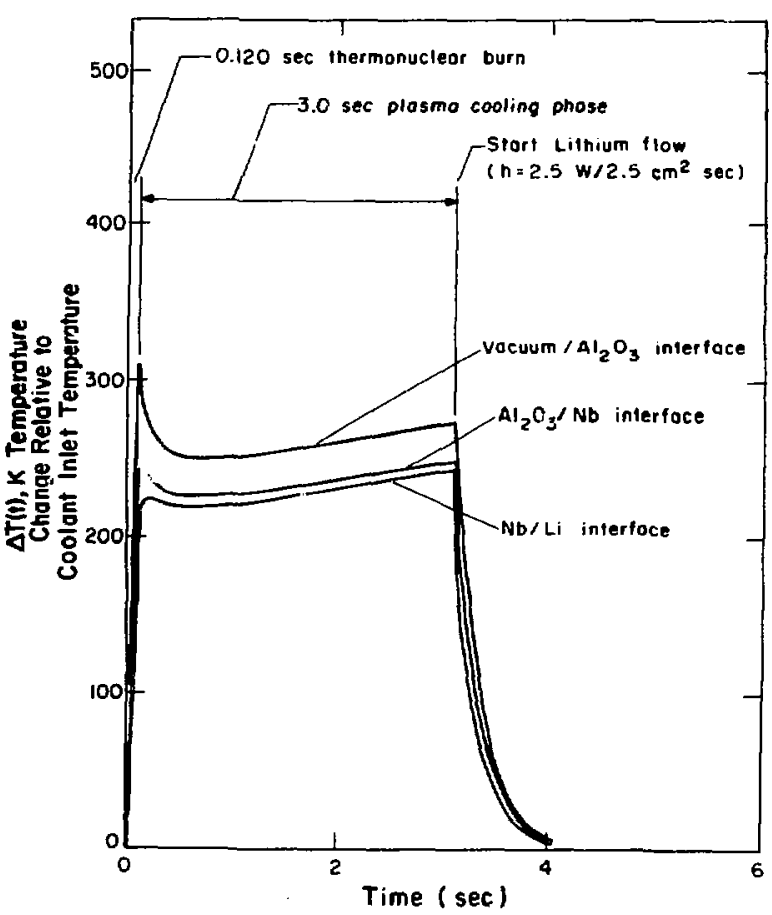

(D)

Fig. 4.4-1.

Temperature transient at first wall for plasma cooling times of $0.5,1.0,2.0$, and 3.0 sec. Period of thermonuclear burn includes rise and fall times. 


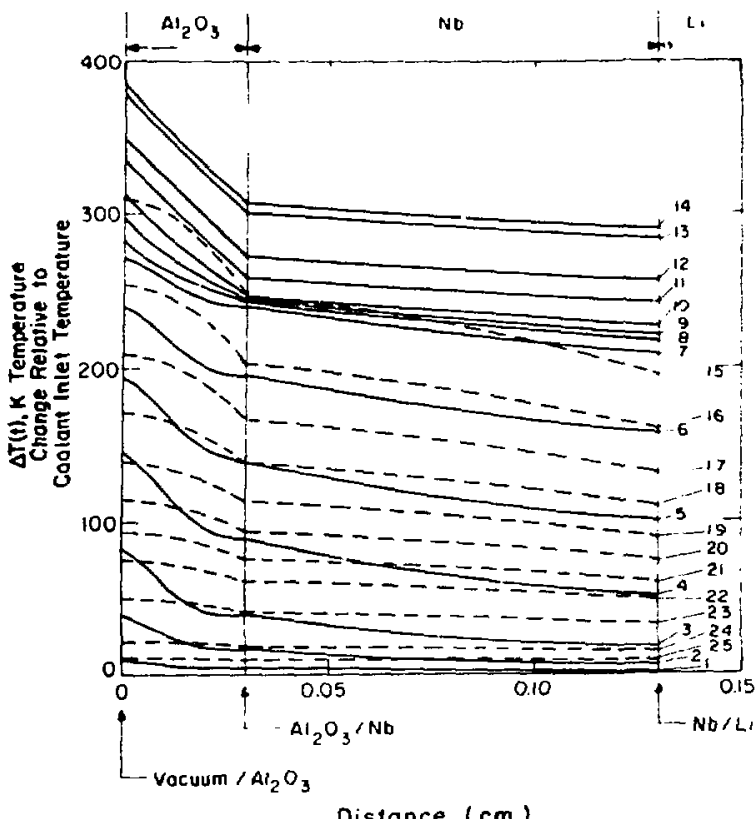

Fig. 4.4-2.

Temperature profile in first wall vs time for various times during the power cycle.

\begin{tabular}{|c|c|c|c|c|c|}
\hline Number & $\begin{array}{l}\text { Time } \\
\text { (Sec) }\end{array}$ & Number & $\begin{array}{l}\text { Time } \\
\text { (Sec) }\end{array}$ & Number & $\begin{array}{l}\text { Time } \\
\text { (Sec) }\end{array}$ \\
\hline 1 & 0.02 & 11 & 0.40 & 21 & 1.45 \\
\hline 2 & 0.03 & 12 & 0.60 & 22 & 1.50 \\
\hline 3 & 0.04 & 13 & 1.00 & 23 & 1.60 \\
\hline 4 & 0.06 & 14 & 1.10 & 24 & 1.80 \\
\hline 5 & 0.08 & 15 & 1.15 & 25 & 2.00 \\
\hline 6 & 0.10 & 16 & 1.20 & & \\
\hline 7 & 0.12 & 17 & 1.25 & & \\
\hline 8 & 0.14 & 18 & 1.30 & & \\
\hline 9 & 0.16 & 19 & 1.35 & & \\
\hline 10 & 0.20 & 20 & 1.40 & & \\
\hline
\end{tabular}

The approximate linearity between the temperature differences in the first wall and the cooling time is also borne out in Fig. 4.4-3. This figure shows results from another one-dimensional heat transfer code using slightly different assumptions, the major assumption being that the bremsstrahlung is absorbed on the insulator surface. From Fig, 4.4-3 it is seen that after the bremsstrahlung peak decays, the temperature difference between plasmainsulator and insulator-metal interfaces is $118 \mathrm{~K}$ for the 0.5 -sec cooling and $62 \mathrm{~K}$ for the 1 -sec plasma cooling time.
To investigate the effect of lithium in removing heat from the first wall, the value of the lithium heat cransfer coefficient was varied. Because of the uncertainty in lithium flow conditions caused by the transient and steady-state magnetic field, several conditions were considered. The condition deemed most realistic assumes a flow rate close to zero during the time the magnetic field is present; the flow tate then increases linearly to a steady-state condition in $0.1 \mathrm{sec}$. During the time that the field is $110 \mathrm{kG}$, calculations indicate that the lithium velocity will actually be about $0.1 \mathrm{~cm} / \mathrm{sec}$, which is finitc but much too low to add turbulence enhancement to the slug flow conditions existing in the blanket. During this period of low but finite flow, the heat transfer coefficient was taken as $0.9 \mathrm{~W} / \mathrm{cm}^{2} \mathrm{~K}$, corresponding to a Nusselt number of 8 . The steady flow heat transfer coefficient was taken as $9.0 \mathrm{~W} / \mathrm{cm}^{2} \mathrm{~K}$, and the transition assumed $\mathrm{t}$ ' occur linearly for $0.1 \mathrm{sec}$ after the plasma cooling field is eliminated. In reality, the lithium velocity will increase in proportion to the decreasing magnetic field, but the increase in flow was not enough to add significantly tu the case of slug flow (Nusselt number of 8 ) used for the lower heat transfer coefficient. The results for this case were shown in Fig. 4.4-3.

Figure 4.4-4A shows temperature results if the heat transfer coefficient is assumed to remain at the low value, even after the magnetic field is decreased to zero. Figure 4.4-4B shows results for the unrealistic case assuming a constant heat transfer coefficient of $9.0 \mathrm{~W} / \mathrm{cm}^{2} \mathrm{~K}$ for the entire cycle time. Finally, Fig. 4.4-4C shows results for the case which allows a inearly increasing heat transfer coefficient, except that the time required to reach the steady flow value is taken as $0.4 \mathrm{sec}$ instead of $0.1 \mathrm{sec}$.

In all cases studied in Figs. 4.4-3 and 4.4-4, it was assumed that the lithium temperature was increased $50 \mathrm{~K}$ by neutron and ganima energy deposition and did nor change thereafter. In practice, temperature transients will vary according to location along the first wall. Near the inlet, the lithium temperature will increase suddenly, but then decrease as cool lithium flows into the channel from the inlet manifold. On the other end of the module, the lithium temperature will continually increase as heated lithium flowing in the channel picks up additional heat from the contiguous structure. The details of the lirhium thermal behavior requires the two-dimensional calculation discussed in Sec. 4.5.

None of the variations presented in Fig. 4.4-1 through 4.4-4 have a significant effect upon the temperature rise incurred from the neutron gamma and bremsstrahlung radiations during the burn. The unrealistically high heat transfer coefficient (Fig. 4.4-4B) does, however, lower the temperature of the niobium-insulator interface, and the 


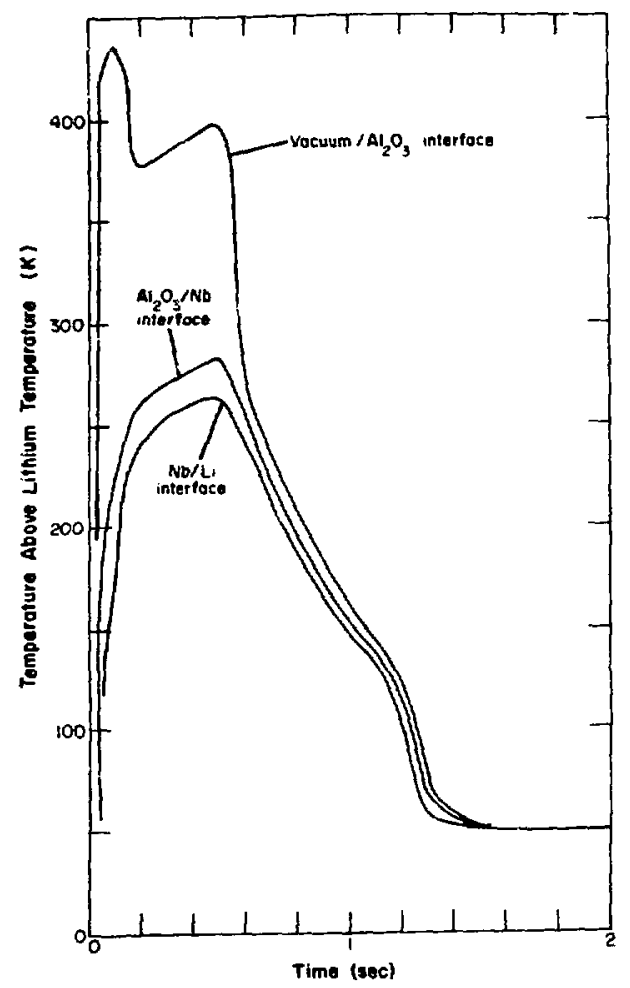

(A)

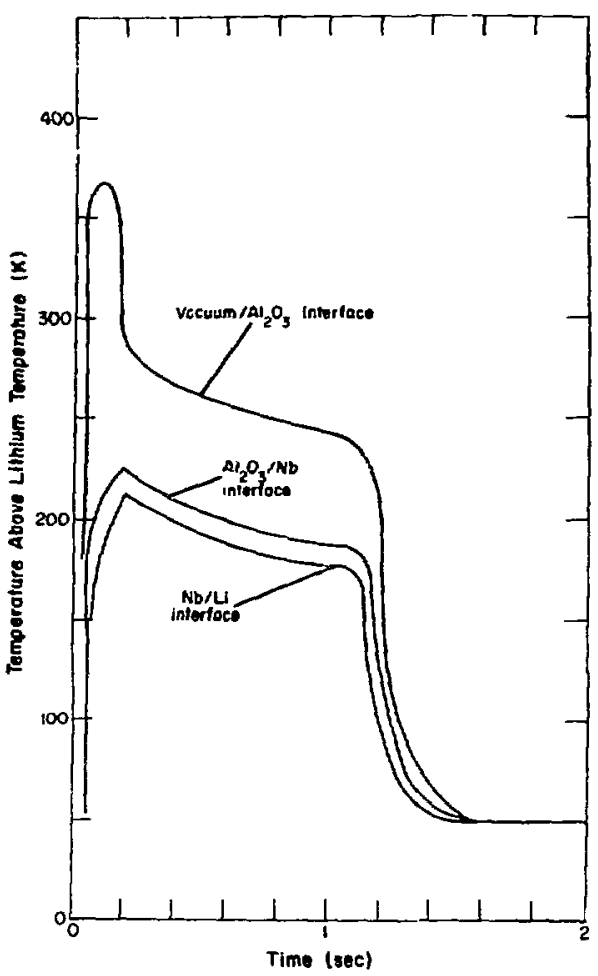

(B)

Fig. 4.4-j.

Temperature transient at first wall for plasma cooling times of 0.5 and 1.0 sec with some convective cooling maintained during the bum. The coolant flow transient is linearly approximated.

cooling time is rapid after the plasma energy is dissipated. On the other hand, the critical temperature difference across the insulator during the plasma cooling is still about $60 \mathrm{~K}$.

Maintaining a heat transfer coefficient corresponding to the slug flow -agime after the plasma cooling indicates that wall cooling occurs after 2.0 sec into the cycle. Little difference is apparent between flow acceleration times of 0.1 and $0.4 \sec$ (Fig. 4.4-3B and 4.4-4C, respectively). The time required to cool the wall to the lithium inlet temperature is increased by the difference in flow acceleration times, i.e., $0.3 \mathrm{sec}$. The results of these calculations with widely different heat transfer coefficients indicate that although the effect is discernible, the lowering of the heat transfer coefficient by the lamination effect of the magnetic field does not cause untoward problems with firstwall cooling by liquid lithium.
The effect of insulator thermal conductivity on the temperature transient were also examined. As one may expect, the temperature difference between plasmainsulator and insulator-metal interfaces during the cooling portion of the cycle were approximately proportional to insulator thickness and inversely proportional to its thermal conductivity.

\subsubsection{First-Wall Stress Calculations}

A thin-shell biaxial stress calculation was made for the case depicted in Fig. 4.4-1B, i.e., plasma cooling time is $1.0 \mathrm{sec}$ and no lithium coolant flow occurs until the plasma has completely transferred its internal energy to the first wall. The tangential stress profile in the first wall is shown as a function of time in Fig. 4.4-5. These stress profiles apply at the axial midpoint of the 1-m-long by 


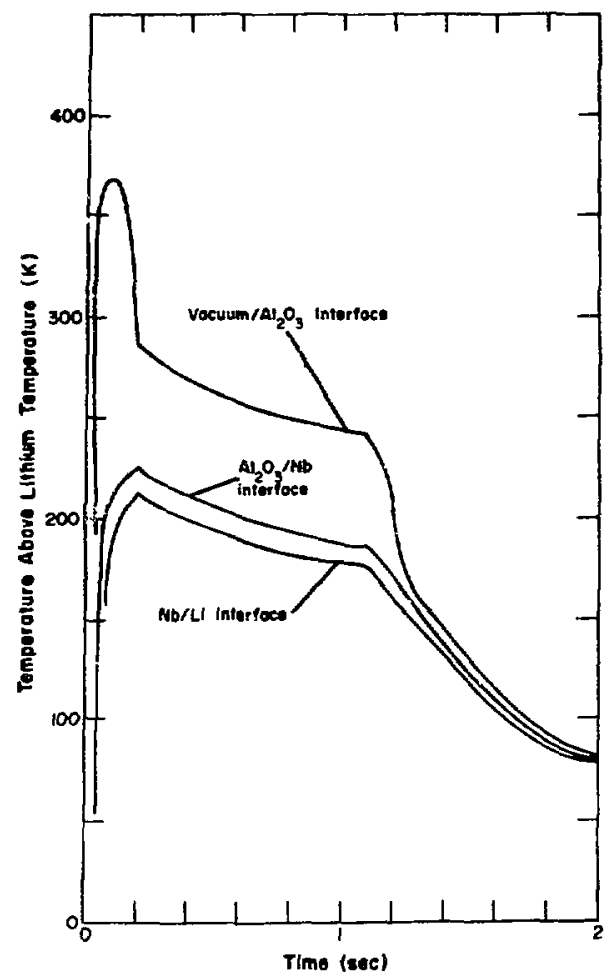

(A)

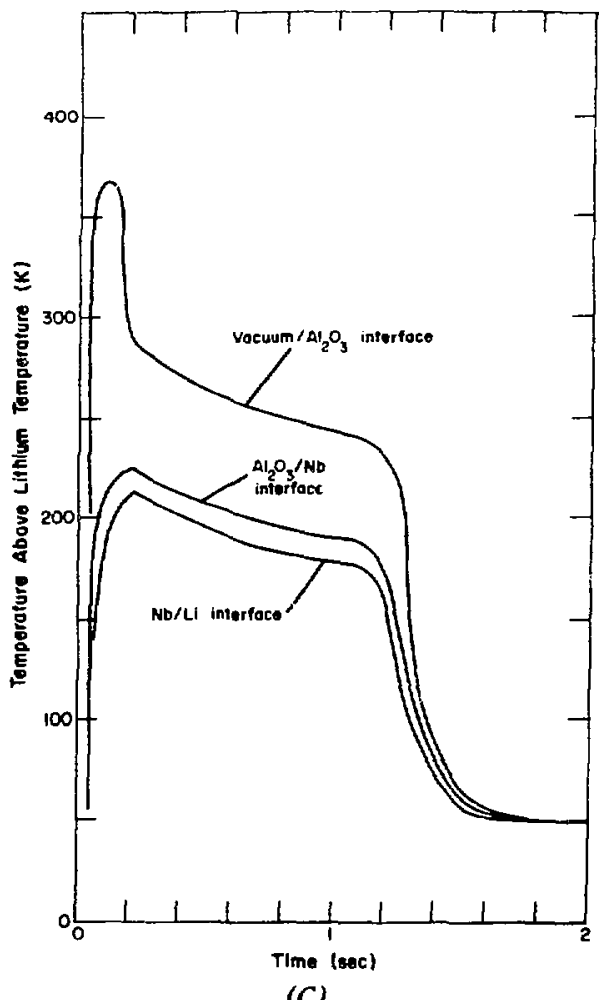

(C)

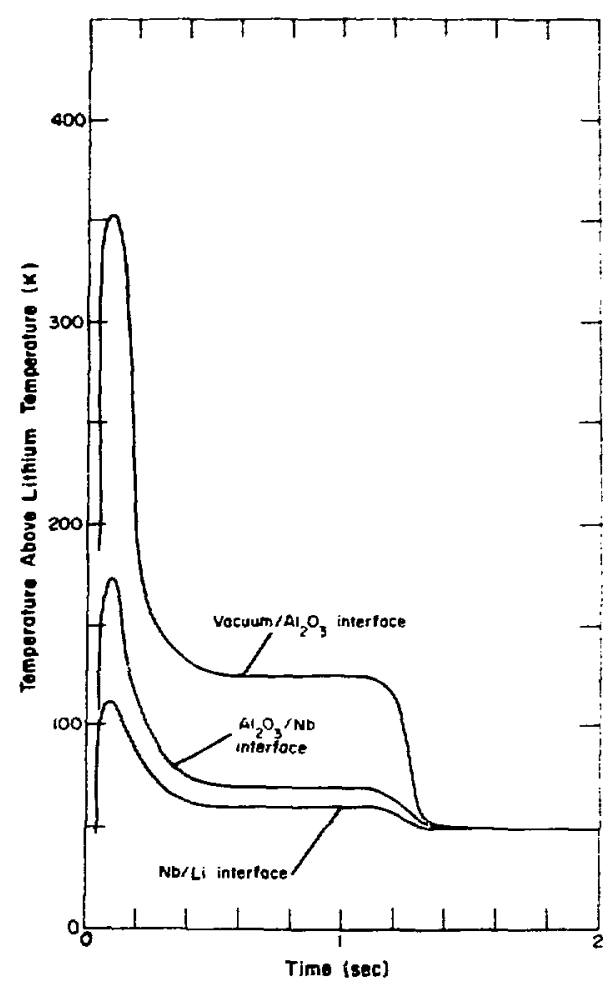

(B)
Fig. 4.4-4.

Temperature transients at first wall for 1.0 -sec plasma cooling time and various conditions placed on beat transfer coefficient.

A. Low valve of beat transfer coefficient.

B. High valve of beat transfer coefficient.

C. Linearly varying value of beat transfer coefficient. 


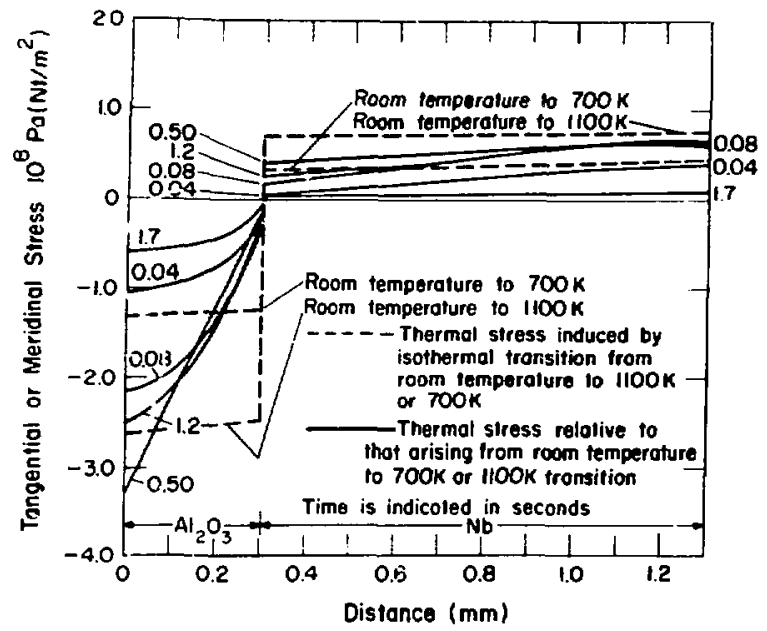

i.ig. 4.4-5.

Stress profile in first wall for the thermal transient depicted in Fig. 4.4-1B.

$0.50-\mathrm{m}$-radius cylinder used in the calculational model; at this point the tangential and meridional stress are nearly equal. Figure 4.4-6 indicates the time dependence of the maximum (compressive) stress in the $\mathrm{Al}_{2} \mathrm{O}_{3}$,

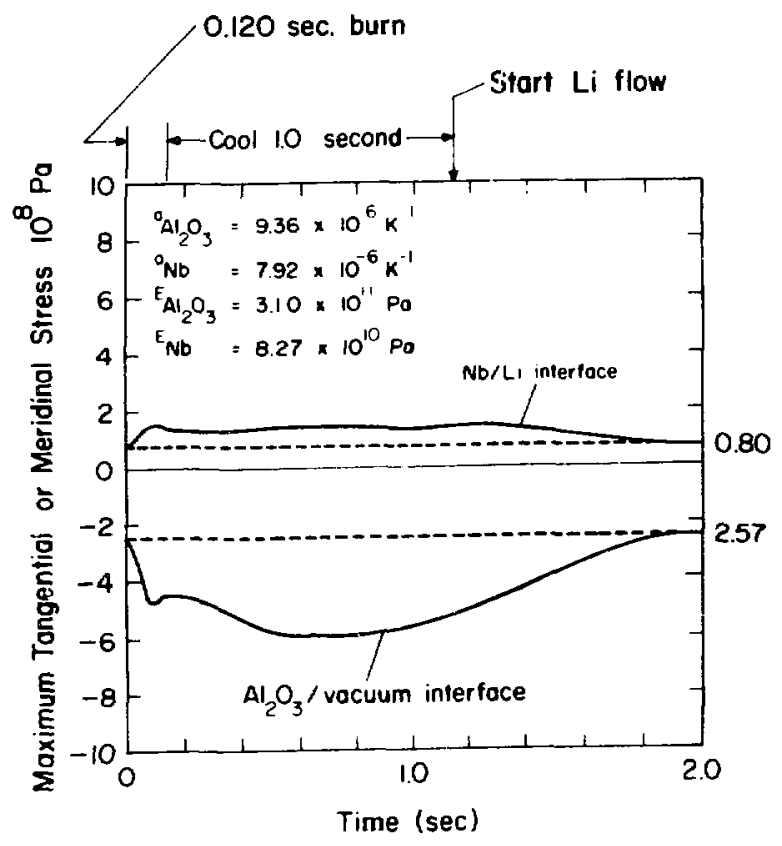

Fig. 4.4-6.

Time dependence of maximum compressive and tensile stress in the first wall for the thermal transient depicted in Fig. 4.4-1B.
$2.57 \times 10^{8} \mathrm{~Pa}$, appears to more than double $\left(6.95 \times 10^{8} \mathrm{~Pa}\right.$ ) during the thermal cycle. The influence of these thermal stresses on first-wall failure mode is discussed in Sec. 4.4.6.2. First-wall stresses induced by lithium flow surges and vacuum forces will be nontrivial, although no detailed calculation of this problem exists; Sec. 4.5.4 addresses this problem to some extent.

\subsubsection{Failure Modes for First Wall}

Failure of the first wall is considered to occur if either its structural role or its electrical role (or both) is no longer fulfilled. The major causes for a potential failure can be related to excessive temperature, thermal gradients, (structural) radiation effects, nuclear transmutations, and chemical effects (including neutral atom sputtering). The following qualitative disclission summarizes the potential impact of probable causes for failure in the context of the RTPR design requirements. Quantitative assessment of radiation effects and sputtering at the first wall is found in Sec. 5.1.

4.4.6.1 First-Wall Failure Induced by Excessive Temperature. During thermonuclear burn, the RTPR design affords little chance for a first-wall failure by means of phase change. The first-wall temperature increases linearly at a rate of $2.3 \mathrm{~K} / \mathrm{msec}$ during the burn; at this rate the burn would have to continue for $\sim 520 \mathrm{msec}$ before the melting point of $\mathrm{Al}_{2} \mathrm{O}_{3}$ is reached (assuming the burn could be sustained that long). The plasma would have expanded to the first wall long before this point; contact of the plasma with the wall will, however, induce local melting.

Another cause for first-wall melting can be related to a plasma cooling stage which is too short. Extrapolating the temperature transients depicted on Fig. 4.4-1, the melting of $\mathrm{Al}_{2} \mathrm{O}_{3}$ surface is expected if the plasma cooling time is less than $\sim 100 \mathrm{msec}$. In principle, the time during which the plasma internal energy $(4.13 \mathrm{MJ} / \mathrm{m})$ is dumped to the first wall is controllable Uncertainties, however, associated with the mechanisms and stability of heat transfer through the neutral gas layer do not permit the elimina. tion of partial melting of the first wall during the plasma cooling stage as a potential first-wall failure mechanism. Work both at LASL and ANL is in progress to elucidate the plasma cooling processes, thereby assuring better control of this geometry. Therefore, structural failure of the insulator (fracture, debonding, localized spallation) nray occur, although the above analysis indicates the thermal

- $10^{8} \mathrm{~Pa}\left(\mathrm{~N} / \mathrm{m}^{2}\right)=14.5 \mathrm{kpsi}$. 
stress level can conceivably be held well below the compressive strength of the insulator $\left(\sim 7 \times 10^{8} \mathrm{~Pa}\right.$ for $\mathrm{Al}_{2} \mathrm{O}_{3}$ ). Furthermore, the creep strength of the underlying metal and the bond strength will play an important role in determining the integrity of the first-wall system under the action of thermal gradients. Stress analyses which incorporate a more realistic geometry and plastic phenomena represent refinements which are not included herein.

In general, thermal gradients within the first-wall system can induce stresses that can lead to fracture, debonding, and spallation. Proper mechanical design, materials selection, bonding techniques, and cooling programming should alleviate these problems. Undoubtedly, extensive testing and calculation of the first-wall system under temperature transients similar to those expected in the operation of the RTPR will be needed to optimize a configuration which can withstand the expected thermal shocks. A solution of this problem appears feasible.

Radiation effects and chemical phenomena represent important $f$ rocesses which may induce failure in the first wall. Section 4 reviews in detail potential failure modes related to radiation damage in the first-wall metal and insulator.

4.4.6.2 First-Wall Failure Induced by Excessive Temperature Gradients. For the condition of a 1 -sec plasma heat dump (Fig. 4.4-1B), temperature gradients in the range of $250 \mathrm{~K} / \mathrm{mm}$ occur in the $\mathrm{Al}_{2} \mathrm{O}_{3}$. As with the problem of excessive temperature, the maximum sustained temperature gradients occur during the plasma cooling stage. Thermal diffusion effects in the $\mathrm{Al}_{2} \mathrm{O}_{3}$ should not present a problem, in that the excessive gradients exist only for $\sim 1$ sec per power cycle. Furthermore, these gradients can be reduced somewhat by extending the period of plasma cooling or by otherwise spreading out the time of the plasma heat dump.

The tangential (and meridional) stresses arising from the temperature gradient (Fig. 4.4-5) amount to a compressive stress of $3.3 \times 10^{8} \mathrm{~Pa}(\sim 50 \mathrm{kpsi})$ in the $\mathrm{Al}_{2} \mathrm{O}_{3}$; this stress is superimposed on a baseline value of $2.6 \times 10^{8} \mathrm{~Pa}(\sim 40 \mathrm{kpsi})$ which results from differential thermal expansion of the $\mathrm{Al}_{2} \mathrm{O}_{3}$ relative to niobium when the first-wall temperature is uniformly raised from ambient values to $: 100 \mathrm{~K}$. Fabrication techniques can be used to relieve the baseline stresses. The burn phase induces a thermal stress of $\sim 1 \times 10^{8} \mathrm{~Pa}(\sim 15 \mathrm{kpsi})$; the remaining $2.3 \times 10^{8} \mathrm{~Pa}(\sim 35 \mathrm{kpsi})$ occurs during the plasma cooling stage. A cooling scheme can probably be devised to reduce this latter stress; in the extreme case, the stress component arising from the plasma cooling can be reduced to nearly zero for cooling times in excess of $\sim 2$ sec. Hence, a minimum value of thermal stress for the burn conditions assumed herein appears to be in the neighborhood of $1 \times 10^{8} \mathrm{~Pa}(\sim 15 \mathrm{kpsi})$ :

\section{REFERENCES}

1. J. Bunch, Los Alamus Scientific Laboratory, privute communicarion, (June 1973).

2. H. Heyes, D. Watson, "Time Lags in the Breakdown of $\mathrm{NaCl}$ at High Temperatures," J. Phys. Chem., 31, 2531 (1970).

3. W. A. Ranken, T. G. Frank, and G. W. Keilholtz, "Effect of Neutron Irradiation on Alumina and $Y$ trois," 3rd Interastional Conference on Thermionic Electrical Power Generation, Julich, Germany. June 1972.

\subsection{BLANKET SYSTEM*}

This section described the neutronics, heat transfer, and magnetic interactions expected to occur within the present blanket design. Many aspects of the blanket design are not opcimal; the purpose of the numerical results presented here is to demonstrate a reasonably consistent and feasible design.

A typical cross section of ihe RTPR core is illustrated in Fig. 3.2-2. As discussed in Section 3.1, each 2-m module is composed of 100 radial sectors to divide the large azimuthal voliages developed during the implosion heating phase. Each radial sector represents an independent flow system, the 100 radial segments being manifolded together for each $2-\mathrm{m}$ module into single lithium inlet and outlet ducts. Figure 4.5-1 illustrates a poloidal cross section of a radial sector showing the relative positions of the blanket structure, plasma, and magnetic coils.

D. J. Dudziak, R. A. Krakowski, LASL; T. A. Coulras, P. J. Persiani, E. Pierson, S. A. W. Gerstl, ANL.

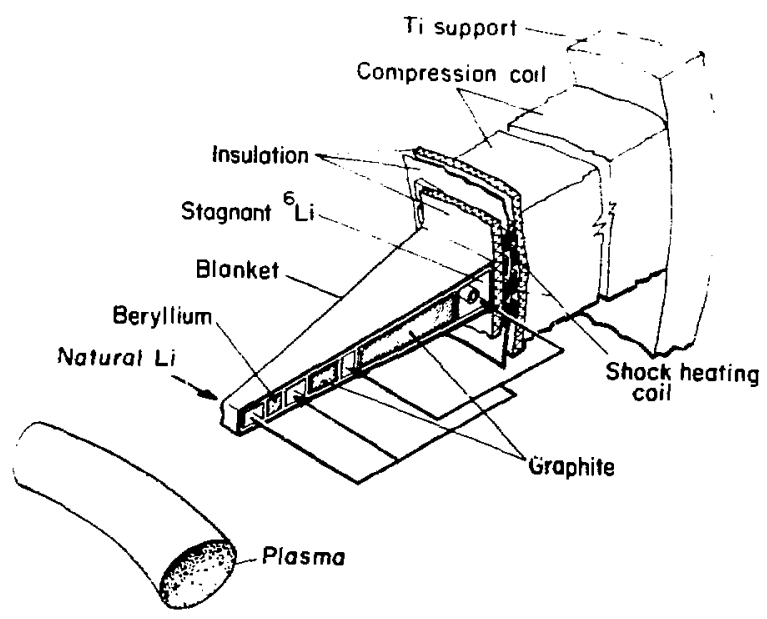

Fig. 4.5-1.

A polodial section of one radial blanket sector illustrating specific blanket regions and litbium flow patbs. 
The countercurrent flow pattern of lithium is aiso shown. The lithium makes i first pass through a given radial segment to cool the first wall, the flow thereafter being diverted to cool the graphite and ${ }^{6} \mathrm{Li}$ regions. The beryllium region is included to multiply the fusion neutrons resulting from $(n, 2 n)$ reactions. Tritium is bred in the two inboard lithium regions by $\left(n, n^{\prime} \alpha\right.$ ) reactions with ${ }^{7} \mathrm{Li}$. Neuzrons which penetrate the ${ }^{7} \mathrm{Li}-\mathrm{Be}$ region are moderated in the graphite region and eventually react in the outboard ${ }^{6} \mathrm{Li}$ region by the ( $n, \alpha$ ) process to produce more tritium. The ${ }^{6} \mathrm{Li}$ region is stagnant, although provisions are made for periodic exchange. The configuration and dimensions selected for the RTPR. blanket represent first-order values, and full optimization of the breeding ratio and thermal aspects of the blanket will be performed at a later stage of the RTPR development. No problems exist, however, in breeding tritium with the 40 -cm-thick blanket model treated herein.

A method of fabrication and assembly of the radial blanket segments is discussed in Sec. 6.2 and illustrated in Fig. 4.5-2. The blanket structure and coolant ducts will be fabricated as one piece, and graphite logs canned is niobium and $\mathrm{Al}_{2} \mathrm{O}_{3}$ will be inserted into the structure. The inlet and return manifolding for each radial sector is also shown on Fig. 4.5-2. Details of the construction and assembly of the core are discussed in Sec. 6.

\subsubsection{System Requirements}

Essential functions of the blanket design are:

1. to breed tritium in sufficient quantities to provide not only tritium for refueling but also to produce sufficient tritium for initial fueling of new D-T reactors;

2. to recover the neutron kinetic and potential (exoergic reaction) energy, as well as bremsstrahlung and residual plasma energy, in the form of high-grade heat which is usable in an efficient, thermodynamic cycle; and

3. to shield the magnetic implosion-heating and compression coils from excessive nuclear heating and neutron radiation damage.

To provide these essential functions, certain requirements are flaced on the blanket design. These requirements are:

1. The blanket must contain sufficient quantities of lithium, either in metallic or ionic form, to accomplish the breeding function. The proportion of enriched lithium, neutron moderator, and ma:prial with appreciable $(n, 2 n)$ cross-section is optimized , yield an acceptable breeding ratio for a minimal blantet thickness. $\Lambda$ minimized blanket thickness reflects a minimized magnetic energy, a point which is crucial for the RTPR.

2. A structurally sound blanket must be sustained under high $\left(\geqslant 10^{22}\right.$ neutrons $/ \mathrm{cm}^{2} /$ year) neutron fluences at temperatures $\sim 1300 \mathrm{~K}$.

3. Provision must be inade to remove the heat generated throughout the blanket by neutron and gamma-ray interactions, bremsstrahlung, and residual plasma energy.

4. The construction of the blanket must permit radial channeling of the magnetic flux inward to the plasma without excessive joule losses.

5. Eiectrical insulation on the niobium first wall must maintain structural integrity and high dielectric strength within $10 \mathrm{sec}$ after an intense burst of neutron, gamma, and bremsstrahlung radiation. This implies that the insulator must have a high thermal conductivity and specific heat to avoid excessive temperature excursions and thermal gradients.

6. Reactor blanket materials which are in contact must be compatible with respect to corrosion, erosion, and other mass transport mechanisms.

7. Provision must be made to accomodate radiationinduced swelling and perhaps even fracture of materials (e.g., in graphite and beryllium).

8. Blanket materials must maintain essential physical properties under severe neutron transmutation of elemental composition and continuous generation of hydrogen and helium.

9. Blanket design must provide for removal of radioactive decay afterheat without structural failure or containment violation in event of loss of coolant.

To accomplish these functions the following blanket design has been adopted.

\subsubsection{Neutronics}

4.5.2.1 Introduction and Summary. Neutron flux, reacion rate, and energy-deposition distributions have been determined for the version of the $\mathrm{RTPR}^{1}$ blanket shown schematically in Fig. 4.5-3. These spectral-spatial data 


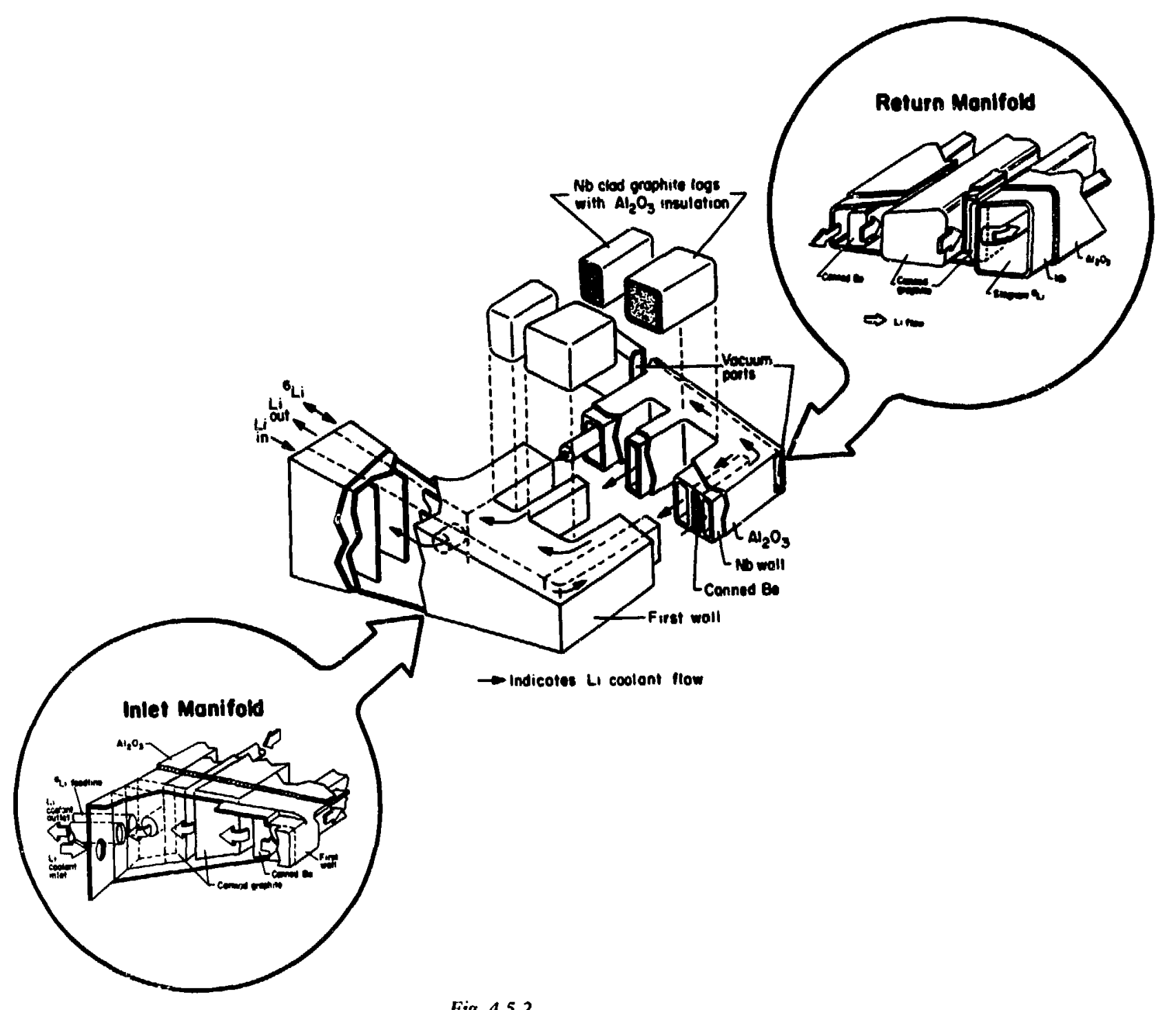

Fig. 4.5.2.

Schematic assemblage of a radial blanket sector showing inlet and returning manifolding of the lirbium coolant. 
have been analyzed to determine the tritium breeding ratio, energy deposition, displacements per atom (dpa). helium production, and neutron transmutation. The RTPR is a staged theta-pinch reactor which has evolved from previous feasibility studies, ${ }^{2.3}$ including neutronics analysis. ${ }^{4,5}$ Figure $4.5-3$ shows a radial traverse of the cylindrical RTPR neutronic model, and Fig. 4.5.4 shows a graph of the 10-MeV neutron flux along this traverse. Material compositions of the regions shown in Fig. 4.5-3 were computed at a nominal $1100 K$ out to $90.9 \mathrm{~cm}$, and $300 \mathrm{~K}$ beyond the alumina thermal insulation. The niobium-alumina segment sidewalls (of Fig. 4.5-1) were the only material homogenized into the regions from 50.0 to $88.9 \mathrm{~cm}$.

The RTPR has a tritium breeding ratio of 1.11 , which gives a doubling time of $\mathbf{4 0}$ days. the mop-up blanket previously used in the neutronic model ${ }^{4,5}$ in place of a concrete shield contributes little to the breeding and has been replaced by conventional radiation shielding in the current design of the RTPR. Helium production and displaced atom rates in the niobium first wall ( $59 \mathrm{ppm} \mathrm{He} / \mathrm{yr}$ and $36 \mathrm{dpa} / \mathrm{yr}$, respectively) are comparable to other CTR designs at the same wall loading, with attendant major concern for radiation damage. Energy

\begin{tabular}{|c|c|c|}
\hline Region Material & No. & Radius \\
\hline Plasmo & 1 & \\
\hline vocuum & 2 & \\
\hline $\begin{array}{l}\text { Alumino Liner } \\
\text { First woll }\end{array}$ & $\frac{3}{4}$ & $\begin{array}{l}4997 \\
500 \\
501\end{array}$ \\
\hline Li Coolant & 5 & \\
\hline & $\frac{6}{7}$ & $\begin{array}{l}531 \\
532 \\
557\end{array}$ \\
\hline & 8 & $\begin{array}{l}557 \\
558\end{array}$ \\
\hline LI Coolant & 9 & \\
\hline$\frac{N b}{\text { Alumina }}$ & $\begin{array}{c}10 \\
11 \\
12 \\
\end{array}$ & $\begin{array}{l}603 \\
604 \\
60.43\end{array}$ \\
\hline Grophite & 13 & \\
\hline \begin{tabular}{|l}
$\mathrm{Nb}$ \\
Aluming \\
$\mathrm{Nb}$ \\
\end{tabular} & $\frac{\frac{14}{15}}{16}$ & $\begin{array}{l}6497 \\
6497 \\
650\end{array}$ \\
\hline Li Coolont & 17 & \\
\hline $\mathrm{Nb}$ & 18 & $\begin{array}{l}\text { E76 } \\
67 ?\end{array}$ \\
\hline \begin{tabular}{|l|} 
Alumina \\
$\mathrm{Nb}$ \\
\end{tabular} & $\frac{19}{20}$ & $\begin{array}{l}677 \\
67.73 \\
6783\end{array}$ \\
\hline Graphite & 21 & \\
\hline NB & 22 & 8257 \\
\hline Alumina & 23 & $\begin{array}{l}9267 \\
827\end{array}$ \\
\hline NS & 24. & \\
\hline Enriched $\sigma_{1,1}$ & 25 & \\
\hline$\overline{N D}$ & 26 & $\begin{array}{l}85.2 \\
85.3\end{array}$ \\
\hline Li Coolont & 27 & \\
\hline$\overline{\mathrm{Ab}}$ & 28 & $\begin{array}{l}863 \\
664\end{array}$ \\
\hline Enriched $6 L_{1}$ & 29 & \\
\hline ND & 30 & $\begin{array}{l}86 \theta \\
869\end{array}$ \\
\hline Alumina & 31 & 909 \\
\hline Impl Cari & 32 & 929 \\
\hline Alumino & 33 & 949 \\
\hline Camp coil & 34 & 1399 \\
\hline$T_{1}$ & 35 & 1349 \\
\hline Alumino & 36 & 1420 \\
\hline
\end{tabular}

Fig. 4.5-3.

Schematic layout of blanket showing specific material dimensions and positions. deposition in the implosion-heating and compression coils is low enough $(\sim 7.2 \mathrm{MJ} / \mathrm{m} /$ pulse) to allow reasonable cooling system paranicters. The energy deposited into the blanket per fusion neutron, $Q_{N}$, svas calculated to be 20.5 $\mathrm{MeV} / \mathrm{n}$. Although this value is large compared to the commonly accepted valves of $16-18 \mathrm{MeV} / \mathrm{n}$, it was used in the overall energy balance (Sec. 2.4). The large value for $Q_{N}$ is attributable in part to the large quantity of be currently being used in the RTPR blanket.

4.5.2.2 Neutronic Model. A one-dimensional cylindrical model of the RTPR blanket shown in Fig. 4.5-3 was used for all analyses. The basic blanket neutronic design is similar to that determined by Bell ${ }^{6}$ for a preliminary design of the RTPR. Because of the high aspect ratio of the torus $^{\circ}$ (major radius/minor radius $=56 / 0.5=112$ ) in the theta-pinch reactor, the assumption of cylindrical geometry should introduce negligible error.

Isotopic ratios for all materials were assumed to be those at beginning of blanket-life (i.e., virgin materials). This assumption is of particular significance for nitural lithium, where preferential depletion of ${ }^{6} \mathrm{Li}$ occurs. In most conceivable fuel management schemes, the ${ }^{6} \mathrm{Li}$ to ${ }^{7} \mathrm{Li}$ ratio will shift during blanket lifetime, with a continuum of values possible. Even the equilibrium case for a simple refueling model with a constant makeup feed of narural lithium has a transient during approach to equilibrium. Solution of the equilibrium concentration problem would require iterative neturonics calculations similar to burnup studies in fission reactors. As can be seen from Table $4.5-1$, the ${ }^{6} \mathrm{Li}$ is depleted at about 4 times the rate of the ${ }^{7} \mathrm{Li}$ depletion.

All neutronics transport calculations were performed with the DTF-IV discrete-ordinates code, ${ }^{7}$ using $S_{4}$ angular quadrature in a one-dimensional cylindical geometry. Cross sections for the nine materials composing the reactor blanket were 100-group, infinitely dilute, $P_{3}$ multigroup values $^{8}$ processed from ENDF-11l by the ETOG code. ${ }^{9}$ The 100-group energy structure consists of 49 groups of 0.1 lethargy width followed by 50 groups of 0.25 lethargy width below $0.11 \mathrm{MeV}$, and a single thermal group below $0.414 \mathrm{eV}$. A volumetric source of $14-\mathrm{MeV}$ neutrons was uniformly distributed in the plasma rcgion, and a vacuum boundary condition was used at the outermost surface $(142 \mathrm{~cm})$. Adequacy of an $\mathrm{S}_{4}-\mathrm{P}_{3}$ analysis to predict an integral quantity such as breeding ratio within $\cong 2 \%$ (as compared to $S_{12}-P_{3}$ ) has been shown previously ${ }^{\mathrm{iO}}$ for a CTR blanket benchmark. Studies by Abdou and Maynard $^{31}$ have demonstrated the sufficiency of a $P_{3}$ truncation of scattering matrices and of the uniform source assumption. In addition $\mathrm{S}_{16}-\mathrm{P}_{3}$ calculations by Muir and $W y$ man, ${ }^{12}$ employing the same 100 -group cross 
TADLE 4.5-1

\section{NEUTRON BALANCE BY REGION AND MATERIAL"}

(Per Fuxion Neutron)

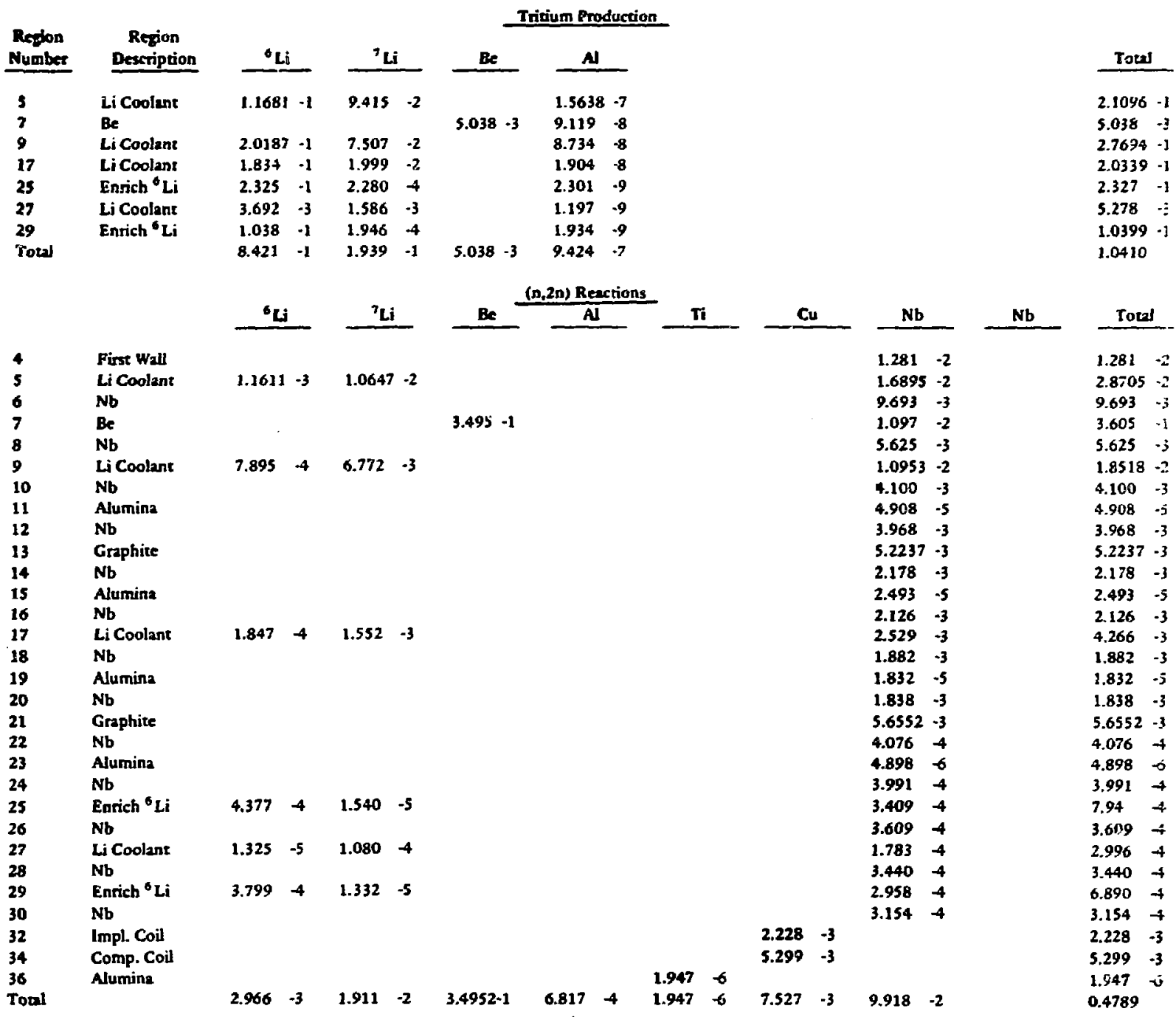


TABLE 4.5-1 (continued)

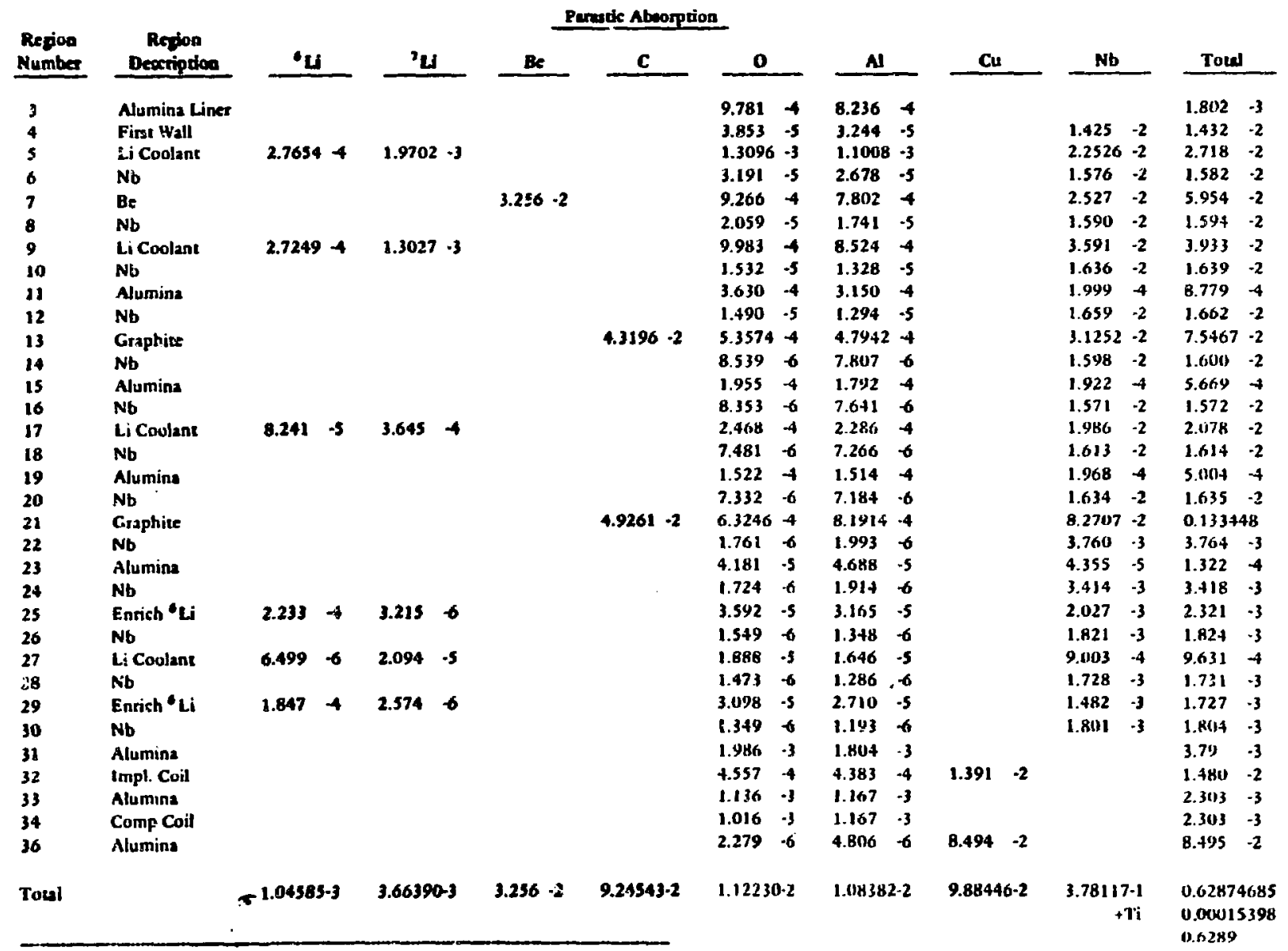

"Negaive number afiet each numerisal value indiates lo mine co that power. 
sections as the present blanket studies, show agreement of computed tritium breeding with integral experiments to within the $10 \%$ estimated experimental error. The integral experiments $^{13}$ were performed in a $30-\mathrm{cm}$-radius (about four mean free paths) sphere of "LiD using a 14-MeV source. Samples of ${ }^{n} \mathrm{Li}$ and ${ }^{7} \mathrm{Li}$ were positioned radially in the sphere and then analyzed for tritium, giving absolute measurements of ${ }^{6} \mathrm{Li}(n, \alpha) \mathrm{T}$ and ${ }^{7} \mathrm{Li}\left(n, n^{\prime} \alpha\right) \mathrm{T}$ reaction rates.

4.5.2.3 Analysis of Blanket Reaction Rates. Neutron flux spatial-spectral distributions computed by the DTF-IV discrete-ordinates code were convoluted with various response functions* to determine reaction rates of interest: e.g., tritium breeding, helium production, displacements per atom (dpa), and kerma**. All fluxes and reaction rates were normalized to a time-averaged first-wall neutron loading (i.e., current of 14.06-MeV neutrons) of $2.0 \mathrm{MW} / \mathrm{m}^{2}$. Because the theta-pinch reactor concept is not particularly sensitive to neutron transport through the outer shield (i.e., no superconducting-coil magnet surrounds the reactor), neutron transport beyond the outer thermal insulation on the titanium was not considered here. Separate calculations of the shielding requirements are presented in Sect. 4.6. Figure 4.5-4 shows the $>10$ $\mathrm{MeV}$ neutron flux distribution through the blanket with a total attenuation of approximately $10^{5}$ from the first wall to the outer boundary. Note from the slope in the compression coil that copper is a good shield for neutrons in this energy range. Figure 4.5-5 shows the total neutron flux distribution through the blanket, normalized to the $2.0 \mathrm{MW} / \mathrm{m}^{2}$ first-wall loading, or $2.81 \times 10^{16} \mathrm{n} / \mathrm{cm}-\mathrm{sec}$ along the torus circumference.

Integral neutron flux spectra are shown in Fig. 4.5-6 for the inner edges of the niobium first wall and the implosion-heating coil. As can be seen, about $19 \%$ of the total flux at the first wall is attributable to $14-\mathrm{MeV}$ neutrons. Also, about $44 \%$ of the total flux at this position results from neutrons with energies greater than 1 $\mathrm{MeV}$. The relatively smaller slopes of both curves between about 3 and $14 \mathrm{MeV}$, as opposed to the 0.2 to $3 \mathrm{MeV}$ region, is caused by the large average energy loss suffered by the 14-MeV neutrons in nonelastic collisions. Therefore, reactions with high thresholds, such as $\mathrm{Nb}(\mathrm{n}, \alpha)$,

-A response function, $F(E)$, is any one of a class of derived coefficients that represents a unit response to a nuclear radiation flux; e.g., activation cross section, dose response, or kerma. If $\phi(E)$ is the flux, $R=0 \int \mathrm{dE} \phi(\mathrm{E}) \mathrm{F}(\mathrm{E})$ is the integrated response.

" Kerma, $K$, is the quotient of $\Delta \mathrm{E}_{k}$ by $\Delta \mathrm{m}$, where $\Delta \mathrm{E}_{k}$ is the sum of the initial kinetic energies of all charged particles liberated in a volume element of a specified material, and $\Delta \mathrm{m}$ is the mass in that volume element.

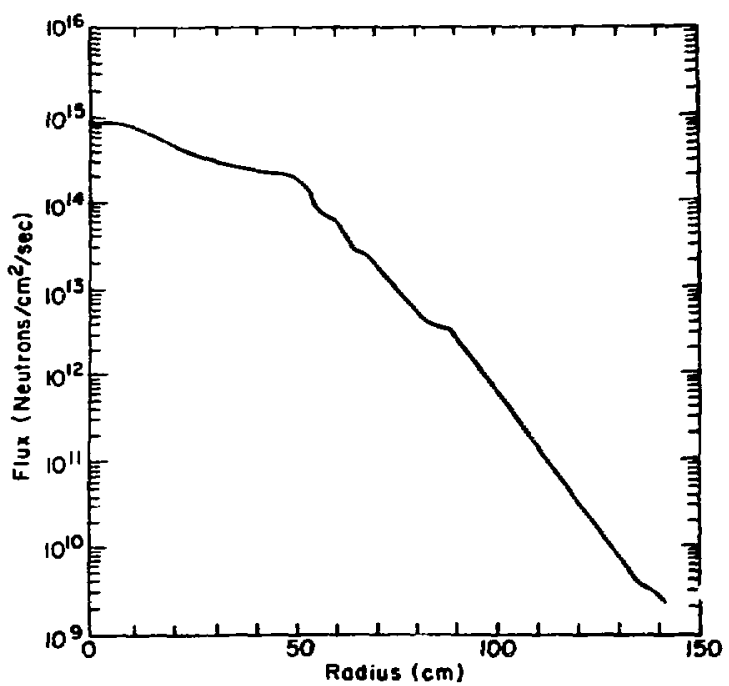

Fig. 4.54.

Neutron flux (energy $>10 \mathrm{MeV}$ ) os radial position for the blanket composition and dimensions given in Fig. 4.5-3.

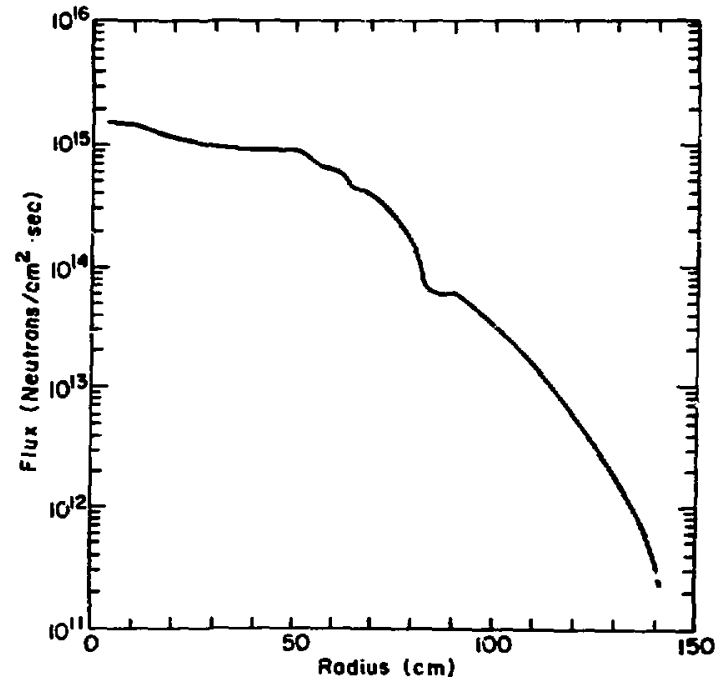

Fig. 4.5-5.

Total neutron flux distribution tbrougb blanket, normalized to $2.0 \mathrm{MW} / \mathrm{m}^{2}$ first wall loading $2.81 \times 10^{16} \mathrm{n} / \mathrm{cm}$-sec seen along torus circumference). 


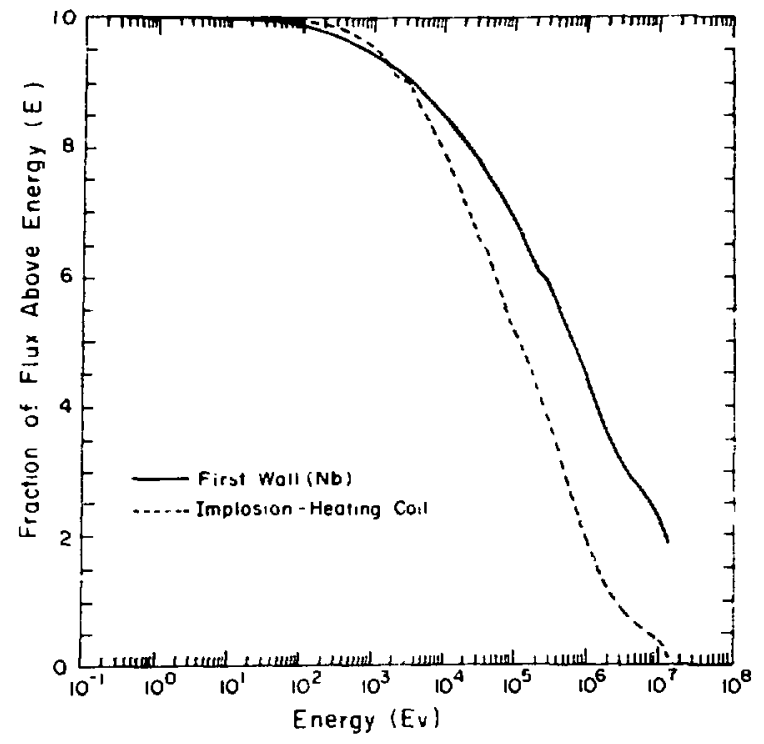

Fig. 4.5-6.

Integral neutron spectra at the inner edges of the niobium first wall and the implosion beating coil.

$\mathrm{Nb}(n, 2 \pi)$, or ${ }^{7} \mathrm{Li}\left(n, n^{\prime} \alpha\right)$, take place predominantly at energies about $14 \mathrm{MeV}$. A flat area in the curve for the copper coil at $\cong 2$ to $3 \mathrm{keV}$ is due to capture resonance in copper.

Breeding Ratio and Doubling Time. A summary of the principal reaction rates is shown in Table 4.5-2, where tritium production, $(n, 2 n)$, and parasitic absorption are given for each blanket region. Ignoring resonance selfshielding gives a total breeding ratio of 1.04 , and previous work $^{5,14}$ indicates that including this effect in niobium will increase the breeding ratio to $\cong 1.11$. Estimation of the self-shielding effect is based upon the assumption that approximately $20 \%$ of the niobium parasitic absorption will be eliminated, as is true in Bell's calculations for a reactor with a similar neutron spectrum. Therefore, from Table 4.5-2, it is seen that the breeding ratio is expected to increase by approximately 0.07 when self-shielding is taken into account.

The definition of doubling time as given by Vogelsang ${ }^{15}$ is adopted here, viz.,

$t_{d}=\frac{I_{e}+I_{b}}{\dot{N}^{-}(T-1)}$ where $T$ is the breeding ratio, $N^{-}$is the rate of tritiurn consumption, $I_{e}$ is the minimum tritium inventory external to the blanket, and $!_{b}$ is the saturated blanket tritium inventory. Assuming one triton consumed for each fusion neruron produced in the plasma, the tritium breeding ratio computed for the RTPR is consistent with the definition

$T=\frac{\dot{\mathrm{N}}^{+}}{\mathrm{N}^{-}}$

where $\dot{\mathrm{N}}^{+}$is the rate of tritium production. Further, the saturated blanket inventory is proportional to the breeding ratio,

$I_{b}=\frac{\operatorname{TN}^{-}}{\lambda_{R}}$

where $\lambda_{R}$ is the removal constant of tritium in the blanket. As given by Burnett et al.' for the RTPR,

$$
\begin{aligned}
& \dot{\mathrm{N}}^{-}=200 \mathrm{~g} / \mathrm{day} \\
& \mathrm{I}_{\mathrm{e}}=2000 \mathrm{~g} \\
& \lambda_{R}=10 / \text { day }
\end{aligned}
$$

For these parameters and any possible values of $T$, the doubling time is determined almost entirely by the tritium inventory external to the blanket; the doubling time is insensitive to the blanket tritium inventory. Substituting the above values in Eq. (r.5-1), the doubling time is 90 days for $T=1.11$.

Analysis of Table 4.5-1 shows the sensitivity of the tritium breeding ratio to not only the lithium cross sections, but also to the $(n, 2 n)$ cross sections of beryllium and niobium. Employing beryllium in a region with a hard spectrum allows the use of a small blanket thickness, thereby decreasing the compression and implosion-heating coil radii and the attendant stored energy, capital costs, joule losses, and eddy losses associated with the pulsed magnetic field. Parasitic absorption is primarily a result of radiative capture in the niobium structure and the copper compression coil, along with $(n, \alpha)$ and $\left(n, n^{\prime}\right) 3 \alpha$ reactions in the graphite. The large neutron leakage into the compression coil is made acceptable by the neutron multiplication in beryllium. Also. the graphite helps reduce this leakage by degrading the spectrum of the flux impinging 


\section{SUMMARY OF HE PRODUCTION, TRANSMUTATIONS, AND DISPLACEMENT DAMAGE IN BERYLLIUM, GRAPHITE, AND COPPER}

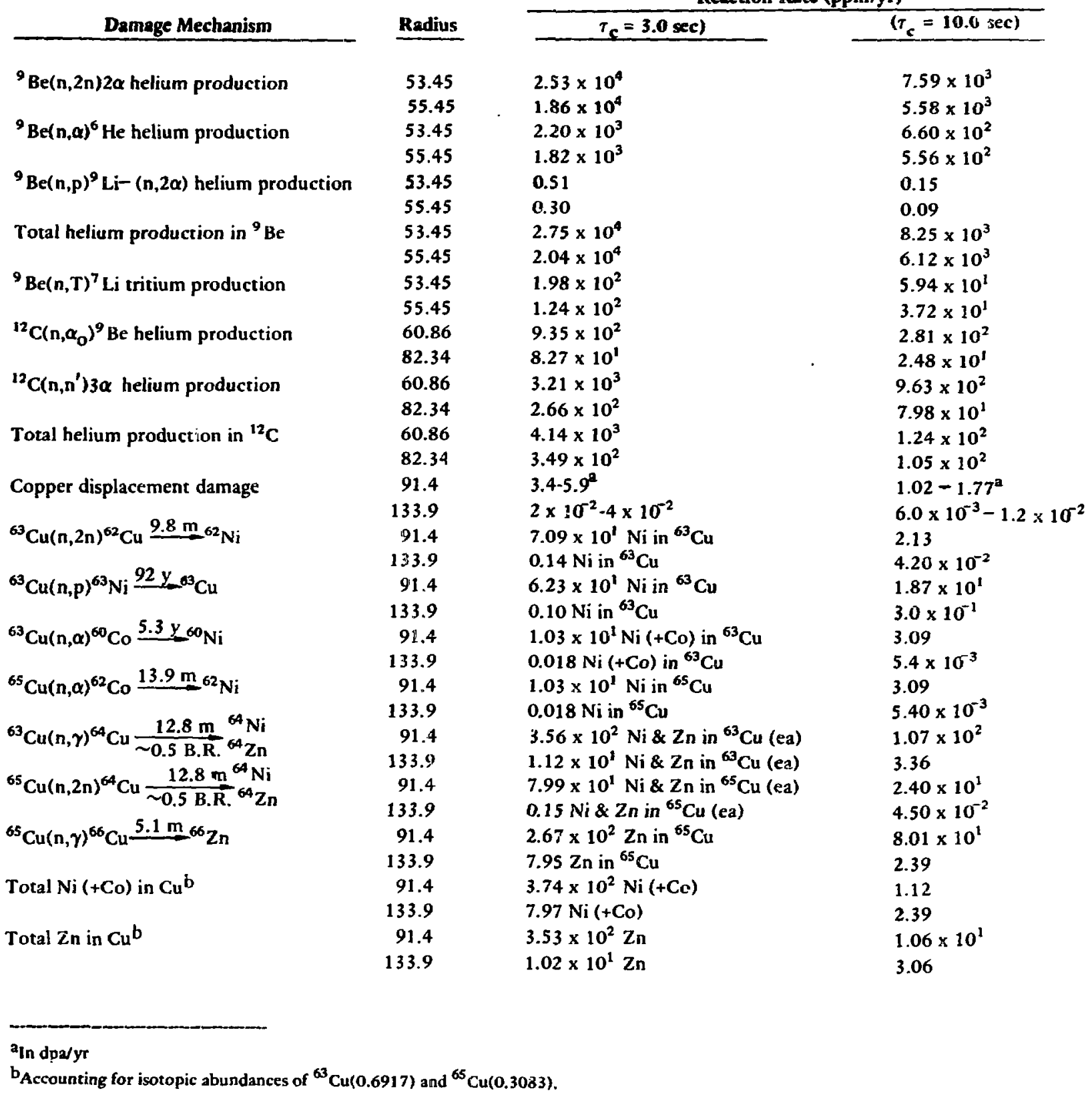


on the enriched lichium region. A general observation can be made concerning the major neutronic differences between the RTPR and the CTR blanket benchmark; ${ }^{10}$ the RTPR is a higher leakage system with much higher neutron multiplication (0.479 vs 0.237 ) and parasitic $a b$ sorption ( 0.629 vs 0.302 ). The net result gives a satisfactory net tritiam breeding.

All neutron transport calculations used a onedimensional blanket model, whereas the actual blanket will have radial penetrations for fuel iujection, ash remoyai, and coolant flow. Because the effects of such inhomogenieties on the breeding ratio cannot be determined precisely, it is of interest to explore the dependence of tritium breeding on parameters which can easily be varied in the one-dimensional calculation. A series of parametric calculations was performed to determine the dependence of the breeding ratio, $T$, on the thickness of specific regions of the blanket, $\Delta R$. The variation of $\Delta R$ was chosen on the basis of the neutron balance tables (Table 4.5-1) with the intent of maximizing the "sensitivity," $d T / d(\Delta R)$. One exception to this procedure is the natural lithium coolant channel situated in the stagnant ${ }^{6} \mathrm{Li}$ region; the thickness was reduced to a value which was judged a more realistic, one-dimensional representation of the $1.25 \mathrm{~cm}$ pipe through the ${ }^{6} \mathrm{Li}$ (see Fig. 4.5-1). Based upon previous studies on a simple RTPR blanket model, beryllium is the most sensitive region. Therefore, the thickness of the beryllium regions was varied, keeping the outer blanket radius constant; the natural lithium region surrounding the beryllium was changed to conserve volume. Likewise, part of the outer (second) graphite regions was diminished in favor of the enriched ${ }^{6} \mathrm{Li}$ region. The results of this sensitivity study are shown in Table 4.5-3. The breeding ratio varies linearly with beryllium thickness in the range 2.0 to $3.5 \mathrm{~cm}$. Some penalty is paid for increasing the breeding ratio this way, in that radiation damage and heating in the implosion-heating and compression coils is increascd slightly beçause of the higher rate of neutron leakage. These studies also indicate, however, that the increased neutron multiplication in the added beryllium also increases the total, recoverable energy per fusion neutron. Although this parametric study is not complete, the design flexibility of achieving desired breeding ratios within the constrairt of relatively thin blankets has been demonstrated and appears well in hand.

Neutron and Gamma-Ray Energy Deposition. The spatial distributions of neutron heating in the blanket structural, moderating, and coolant materials have been determined. The energy deposition from direct neutron interactions, not including gamma-ray cnergy, is shown in Fig. 4.5-7. Multigroup kerma factors for these calculations

TABLE 4.5-3

RTPR BREEDING STUDY

\begin{tabular}{|c|c|c|c|c|c|}
\hline \multirow[b]{2}{*}{ Region (no.) } & \multicolumn{5}{|c|}{ Thicknesses $(\mathrm{cm})$} \\
\hline & Case 0 & Case 1 & Case 2 & Case 3 & Case 4 \\
\hline Lithium coolant (5) & 3.0 & 2.5 & 2.5 & 3.0 & 3.0 \\
\hline P,e (7) & 2.5 & 3.0 & 3.5 & 2.0 & 2.0 \\
\hline Lithium coolant (9) & 4.5 & 4.5 & 4.0 & 5.0 & 5.0 \\
\hline Secund graphite (21) & 15.0 & 14.0 & 13.5 & 13.5 & 15.0 \\
\hline Enriched ${ }^{6} \mathrm{Li}(25)$ & 2.4 & 3.4 & 3.9 & 3.9 & 2.4 \\
\hline Lithuum coolant (27) & 1.0 & 0.3 & 0.3 & 0.3 & 1.0 \\
\hline Enriched ${ }^{6} \mathrm{Li}(29)$ & 2.4 & 3.1 & 3.1 & 3.1 & 2.4 \\
\hline Breeding ratio $^{a}$ & 1.04 & 1.08 & 1.12 & 1.02 & 1.01 \\
\hline Doubling time (days) ${ }^{b}$ & 90 & 65 & 55 & 110 & 125 \\
\hline
\end{tabular}




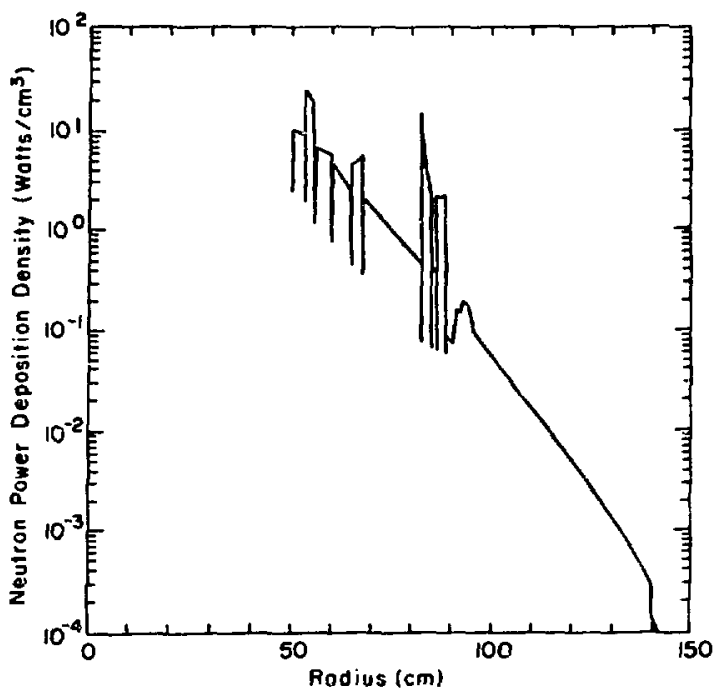

Fig. 4.5.7.

Spatial distribution in blanket of beating rates resutting from neutron beating. $\left(\tau_{c}=10 \mathrm{sec}\right)$

were taken from Ref. 16 where possible; they include kinetic energies imparted to the struck nucleus and to the charged particles emitted. Figure 4.5-7, therefore, presents only one component of the total energy deposition, and in fact a major portion will result from gamma heating as shown in Fig. 4.5-8.

At the reference $2.0 \mathrm{~mW} / \mathrm{m}^{2}$ current of primary 14-MeV neutrons incident on the first wall, total neutron heating per pulse in the implosion heating and compression coils is $0.75 \mathrm{MJ} / \mathrm{m}$. Total gamma heating at 6.42 $\mathrm{MJ} / \mathrm{m}$ per pulse, is almost an order of magnitude higher than the neutron heating. Heating of the compression coil is of considerable importance because of heat removal requirements. Remcval of this heat load, however, is well within present coolant technology (see Section 4.2).

Detailed calculations of the spatial distribution of gamma energy deposition in the blanket were also performed. Gamma-ray production cross sections in the ENDF files ${ }^{17}$ have been processed by the LAPHANO code $^{18}$ into 100 - by 21 -group production matrices, using the gamma-ray energy group structure of Ref. 16. Gamma-ray interaction cross sections from the ENDF files have been processed into the same 21-group structure by use of the GAMLEG code. ${ }^{19}$ Gamma-ray transport calculations were performed with the same DTF-IV discrete-ordinates code used for the neutron transport calculations.

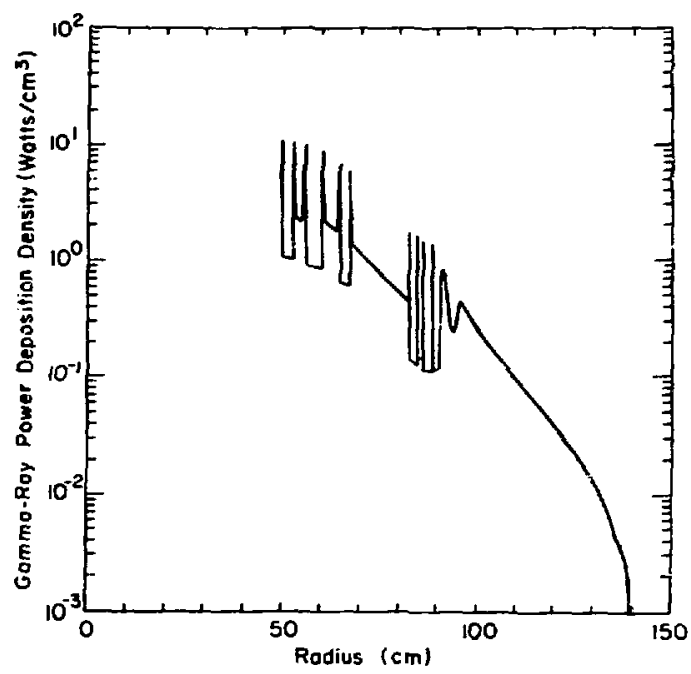

Fig. 4.5.8.

Spatial distribution in blanket of beating rates re sulting from gamma beating. $\left(\tau_{c}=10 \mathrm{sec}\right)$

Radiation Damage. As with most CTR concepts, radiation damage to the first wall and other components of the RTPR represents a major design consideration. Helium. production and displacement-per-atom (dpa) rates have been accordingly determined for the niobium structure, assuming a $2.0 \mathrm{MW} / \mathrm{m}^{2}$ timie-averaged wall loading. These two damage mechanisms are considered by metallurgists to be of crucial importance to properties such as ductility and dimensional stability. Fibure 4.5-9 presents the results of computations predicting these damage rates in niobium structure wherever it occurs throughout the blanket.* The peak values at the first wall, $59 \mathrm{ppm} \mathrm{He} / \mathrm{yr}$ and $36 \mathrm{dpa} / \mathrm{yr}$, compare with values of $54 \mathrm{ppm} \mathrm{He} / \mathrm{yr}$ and 42 $\mathrm{dpa} / \mathrm{yr}$, respectively, for a reference Tokamak reactor ${ }^{20}$ adjusted to the same wall loading.

Using recent displacement damage functions, ${ }^{21}$ the computed displacement damage is approximately the same as that for structural materials of a typical experimental fast breeder (fission) reactor, ${ }^{20}$ EBR-H. However, possible synergistic effects of displacement damage and much higher helium production in CTRs is still unknown.

These results are independent of the slight homogenization used for the neutronic model, except the homogenization a?fects the neutron energy spectrum in the niobium. 


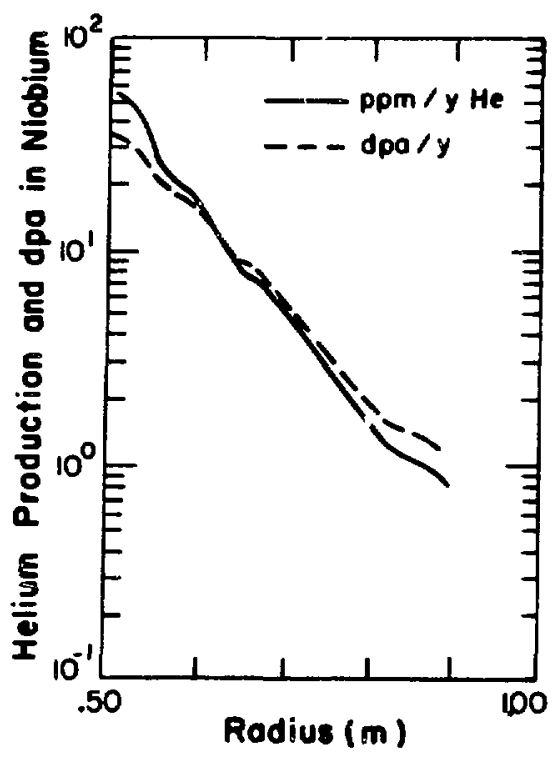

Fig. 4.5-9.

Spatial distribution in blanket of displaced atoms and helium production fo: niobium. $\left(\tau_{c}=10 \mathrm{sec}\right)$

As can be seen in Fig. 4.5-9, softening of the neutron spectrum within the blanket decreases the helium ppmto-dpa ratio by a factor of over two, but this lower ratio is still about a factor of five greater than for a fast breeder reactor core. This problem is addressed in detail in Section 5.
The displacement damage functions used for these RTPR calculations ${ }^{21}$ differ from previous values in that the newer values treat recoil atom spectra from $(n, 2 n)$ reactions in more detail. Proper treatment of the $(n, 2 n)$ reaction is especially important for energies near $14 \mathrm{MeV}$ where the cross section is more than 1 barn and in excess of the inelastic scattering cross section of $\cong 0.9$ barns.

Also of considerable importance for a theta-pinch based reactor is the radiation damage to electrical insulators. Insulators for the RTPR can be categorized according to severity of radiation environment and the associated demands upon the insulating properties in this environment. To evaluate the reference insulator, alumina $\left(\mathrm{Al}_{2} \mathrm{O}_{3}\right)$, transmutation products were considered for all cases where production cross sections were available, and equilibrium concentrations determined for the radioactive species ${ }^{28} \mathrm{Al},{ }^{27} \mathrm{Mg},{ }^{24} \mathrm{Na}$, and ${ }^{16} \mathrm{~N}$. Table $4.5-4$ presents a summary of the transmutation product buildup in the first-wall alumina for a reference wal! loading of $\mathbf{2 . 0}$ $\mathrm{MW} / \mathrm{m}^{2}$. Equilibrium radioactive species had negligible $(<10 \mathrm{ppm})$ concentrations. The hydrogen concentrations in Table 4.5-4 include a!l three isotopes, but total tritium production was less than $1 \mathrm{ppm}$. A list of all the transmutation reactions considered is given as a footnote to Table 4.5-4. Cross sections were derived from ENDF-III, and no account was taken for second-order transmutation. Of importance to the material properties is the unequal burnout of aluminum and oxygen, rendering a net shift toward a hyperstoichiometric alumina (oxygen excess of $\sim 220 \mathrm{ppm} / \mathrm{yr}$ ).

TABLE 4.5-4

\section{TRANSMUTATION PRODUCT BUILDUP IN ALUMINA FIRST-WALL INSULATOR}

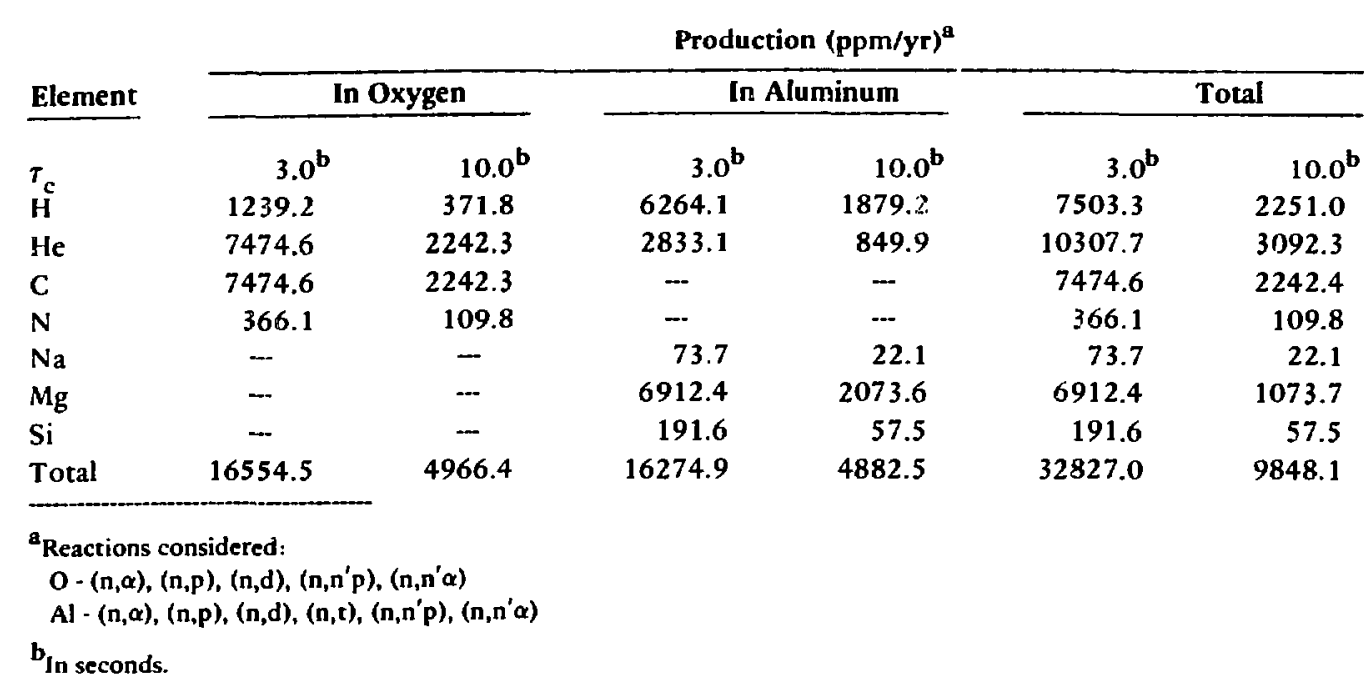


Helium production in beryllium and carbon (graphite) portend serious radiation swelling and gas pressure generation. Hence, production rates have been computed for these two materials. As can be seen in Table 4.5-2, beryllium has rates varying from $8250 \mathrm{ppm} / \mathrm{yr}$ to 6120 $\mathrm{ppm} / \mathrm{yr}$, decreasing becween these two values approximately exponentially with radius. Corresponding rates in the graphite region are $1240 \mathrm{ppm} / \mathrm{yr}$ to $105 \mathrm{ppm} / \mathrm{yr}$, again varying approximately exponentially with radius. Also, sritium production in beryllium varies from 60 to 37 ppm/yr.

Radiation damage to the copper windings of the implosion-heating, and compression coils is of concern because of effects on electrical conductivity as well as structural properties. Displacement per atom were estimated from available data for niobium and vanadium to determine an order of magnitude of the damage. These results, along with detailed transmutation calcuiarions, are summarized in Table 4.5-2. Transmutation occurs approximately equally to nickel and zinc, changing the properties of the copper. These effects are discussed in more detail in Sec 5.

\subsubsection{Induced Radioactivity}

Lithium Coolant. The requirements for shielding, instrumentation, storage, and handling of lithium necessitate a detailed study of the radioactivity induced in the coolant and blanket structural materials. The specific radioactivity of lithium results from one or more of the following mechanisms.

1. The isotopes initially present as impurities in lithium are activated by the neutron flux during passage through the reactor.

2. A fraction of the surface isotopes of the structural material in the reactor section and in contact with the lithium are sputtered or recoiled into the coolant from a capture or scattering reaction.

3. Introduction of radioactive and nonradioactive isotopes into the coolant as a result of corrosion processes.

Based on these mechanisms the specific activitics in the lithium coolant are estimated in Vol. 11 .

Uncertainties in the computed estimates arise from inadequate information on the impurity content, crosssection data, rates of corrosion, sputtering yields, etc. Typical impurities to be expected in lithium are given in Table 4.5-5. The several thousand ppm of fluorine and chlorine content in lithium result from the proposed technique of molten salt extraction of LiT from lithium using a $\mathrm{LiF}-\mathrm{LiCl}$ eutectic. More importanty, the radioactive constituents also to be expected in lithium result from the impurities in the $\mathrm{Nb}-1 \% \mathrm{Zr}$ conduit system are slimmarized in Table 4.5-6. The actual content of these isotopes in lithium as dissolved species and as suspended parriculate will depend on the corrosion and dissolution properties of the alloy in the lithium coolant. This is presently unknown and estimates are thus highly uncertain.

Other factors which contribute to these uncertainties are:

1. Corrosion seldom occurs at a continuous rate; films may form which may inhibit further corrosion or the corrosion process may be accelerazed if scales are formed and then flake off.

2. Activation time is difficult to determine since some dissolved or suspended materials may selectively deposit out in an active area and then may be discharged into the coolant stream at some later time.

TABLE 4.5-5

\section{TYPICAL IMPURITIES FOUND IN HIGH PURITY LITHIUM}

\begin{tabular}{|c|c|c|c|}
\hline Element & Content (ppm) & Element & Content (ppri) \\
\hline Si & $5-50$ & $\mathrm{Ni}$ & 100 \\
\hline $\mathbf{O}$ & 100 & $\mathrm{Cr}$ & 100 \\
\hline $\mathbf{N}$ & $30-500$ & $\mathrm{Ta}$ & 100 \\
\hline $\mathrm{Ca}$ & 100 & $\mathbf{F}$ & 3000 \\
\hline $\mathrm{Na}$ & 100 & $\mathrm{Cl}$ & 3000 \\
\hline $\mathbf{K}$ & 100 & $\mathrm{Nb}$ & - \\
\hline $\mathrm{Fe}$ & 100 & - & - \\
\hline
\end{tabular}

TABLE 4.5-6

\section{TYPICAL IMPURITIES PRESENT IN COMMERCIAL Nb-1\% Zr ALLOY}

\begin{tabular}{|c|c|c|c|}
\hline Element & Content (ppm) & Element & Content (ppm) \\
\hline$w$ & 300 & $\mathbf{0}$ & 300 \\
\hline Mo & 100 & $\mathbf{N}$ & 200 \\
\hline $\mathrm{Ta}$ & 1,000 & $\mathbf{H}$ & 20 \\
\hline Hf & 100 & C & 100 \\
\hline $\mathrm{Zr}$ & 10,000 & $\mathrm{Fe}$ & 50 \\
\hline Nb & 985,000 & $\mathrm{Ni}$ & 20 \\
\hline
\end{tabular}


3. Much of the activity is associated with solids which may settle out in low-velocity areas.

4. The formation of oxide films on metal surfaces affects subsequent corrosion and recoil processes.

5. All of the nuclides are not subjected to the same flux.

An example of what may be expected in fusion power reactor coolant systems may be obtained from light-water power reactor (LWR) experience. Measurements on a coolant water activity indicate that for stainless steel and zirconium alloy cladding systems, the major source of activity is comprised of the ${ }^{60} \mathrm{Co},{ }^{58} \mathrm{Co},{ }^{59} \mathrm{Fe},{ }^{57} \mathrm{Cr}$, and ${ }^{54} \mathrm{Mn}$ isotopes. These result mainly from the corrosion of the cladding and structural materials. Of these, the longlived ${ }^{60} \mathrm{Co}$ and ${ }^{58} \mathrm{Co}$ account for about $50 \%$ of the radioactivity found in boiling water and pressurized water reactors. The ${ }^{60} \mathrm{Co}$ results from the thermal neutron reaction, $\left.{ }^{59} \mathrm{Co}(n, p)\right)^{60} \mathrm{Co}$, with the cobalt impurity present in the cladding material. The ${ }^{58} \mathrm{Co}$ is produced by the fast neutron reaction, ${ }^{58} \mathrm{Ni}(\mathrm{n}, \mathrm{p}){ }^{58} \mathrm{Co}$, on the nickel content in the cladding alloy. The published results of measurements on existing reactor power systems seem to indicate that the difference in the isotopic contribution depend not only on neutron spectrum but also on the concentration of impurities in the clad. This is particularly true for systems where the coolant-borne activity is due mainly to the corrosion of radioactive isotopes from the cladding surface.

Structural Materials. In handling and storing irradiated components of the reactor, such as removable assemblies and structural members, problems will arise that require a thorough understanding of the radioactivity induced in the commercially available material involved.

An indication as to the elements present in a material, either as alloys or impurities, responsible for the activity, and the type of radjation emitted by the activity is necessary if an efficient design of shielding and storing facilities is to result.

In many commercial materials it is possible to reduce neutron-induced gamma activity by decrcasing the content of one or more impurities. However, there are many factors to consider before setting up preliminary specifications. Some of these factcrs are the period of irradiation, the time after irradiation at which the material is to be handled, and the feasibility of decreasing the amount of an impurity.

To better assess the long-range consequences of waste management of radioactive structures, a thorough analysis accounting for all impurities in the calculated estimates will be mainly dependent upon two factors: (1) the qualitative and quantitative analysis of the impurities present in the material; and (2) the analysis of the reactions and radiation emitted.

The radioactivity induced in the copper coils is expected to be as severe a handling problem as for the structural components. This problem is also treated quantitatively in Vol. II.

The degree of accuracy required in determining the "impurities" and their amounts present is largely dependent upon the neutron flux in which the material is to be irradiated. Impurities present in micrograms per gram of material become important when irradiated in a high-flux reactor. If the quantitative analysis of an impurity, which is one of many major contributors to the total induced activity, is in error, then the corresponding error in the total intensity is of a lower magnitude. If the radioactivity dictates the choice of material, then the absence of certain isotopes must be definitely established in the material specifications.

\subsubsection{Heat Transfer and Thermal Response of the Blan-} ket

Because of the pulsed character of the RTPR, the heat transfer in the blanket is cyclical. Two crucial demains of blanket heat transfer can be identified: (1) the transient, start-up period when the time-averaged temperature of each blanket region approaches a constant value; the transient period starts from an initial temperature which is determined by the degree of blanket and coolant preheating, (2) a steady-state regime where the temperature of each region oscillates about the time-averaged value. The most crucial portion of the RTPR thermal response deals with the first wall and has been already discussed in Sec. 4.4.4. Thermal problems in regions other than the first wall appear to be less serious in that considerably more design flexibiity exists and materials failure in other parts of the blanket are not as devastating to the basic operation of the RTPR. Within the constraints established by tritium breeding, a major objective of the blanket design is to minimize the amount of coolant which must be circulated throughout the power cycle. 
The interaction of the lithium flow with magnetic fields, although having profound influence on the heat transfer characteristics, is treated in Sec. 4.5.4 and 4.5.5. As previously discussed (p. 2 ) the vacuum time constant establishes a lower limit to the rate at which the RTPR can be pulsed $\left(\tau_{c} \sim 3.0 \mathrm{sec}\right)$. Although the design value for $\tau_{c}$ was chosen to be $10.0 \mathrm{sec}$ (established by annual neutron fluences at the first wall), the blanket heat transfer calculations have used $\tau_{c}=\widetilde{\tau}_{c}(3.0 \mathrm{sec})$ as indicative of a worst case for heat removal requirements.

As a restilt of the large pressure drop sustained by lithium flowing across magnetic fields lines, the flow of coolane virtually ceases during the thermonuclear burn portion of the power cycle. To minimize the quantity of coolant circulating through the reactor, the flow rate chosen for these preliminary calculations yields a residence or "hoid-up" time for lithium in the blanket equal to the coolisg time, $2.0 \mathrm{sec}$ ). Therefore, the volumetric flow rate of lithium is equated to $V_{L_{i}} / \tau_{c}$, where $V_{L i}$ represents the volume of flowing lithium contained by one radial blanket segment $\left(0.72 \mathrm{~m}^{3}\right)$.

Referring to Figs. 4.5-1 and 4.5-2. lithium at inlet conditions is directed past the first wall for each $2 \cdot \mathrm{m}$ segment of torus. The coolant is then split by an appropriately designed manifold and directed in parallel through the beryllium-graphite channel, the graphitegraphite channel, and the ${ }^{6} \mathrm{Li}$ region. In practice, the return flow rate will be metered by appropriate orificing to insure that the lithium contained by each channel has identicai residence times.

This kind of orificing is achieved in the numerical calculations by distributing the volumetric flow in accordance to the fractional area presented by each channel for retum flow. Other flow schemes and configurations probably can be found to optimize the efficiency of blantict heat removal. The computations contained herein, however, are intended to fumish only a first-order estimate of the thermal response expected of the blanket; more extensive iteration on the thermal, hydraulic, and neutronic portions of the composite blanket design will be required before a design is finalized.

Table 4.5-7 summarizes the RTPR blanket regions, dimensions, and energy (gamma and neutron) deposition distributions. The temporal behavior of the gamma and neutron energy sources follows the thermonuclear burn dynamics depicted in Figs. 2.2-1 and 2.2-2. Additionally, the bremsstrablung deposition into the first-wall insulator and metal, as well as the heat flux at the first wall originating from the plasma cooling phase, coitribute to the thermal response of the blanket. A total of $93.1 \mathrm{MJ} / \mathrm{m} / \mathrm{pulse}$ of gamma ray and neutron energy enter tive blanket; $32.7 \%$ into the lithium coolant, $30.6 \%$ into the graphite, $23.3 \%$ into the beryilium. $6.6 \%$ into the stagnant ${ }^{6} \mathrm{Li}$, and the remaining $6.8 \%$ into the blanket structure $\left(\mathrm{Al}_{2} \mathrm{O}_{3}\right.$ and niobium). As observed from Figs. 4.5-7 and 4.5-8, a strong spatial dependence within a given region exists; this spatial dependence is included in all heat transfer calculations when appropriate.

To estimate the rime-dependence of the blanket temperature distribution, a two-dimensional heat transfer code was programmed to represent a single, 2-m-long blanket segment. Figure 4.5-11 illustrates the finiteelement geometry used to peiform this 11 region, axisymmetric calculation. The results of the thermonuclear burn salculations (Figs. 2.2.1 and 2.2-2) were used to generate first-wall heat fluxes during that portion of the 
TABLE 4.5-7

BLANKET GEOMETRY AND ENERCY DEPOSITION

\begin{tabular}{|c|c|c|c|c|c|c|}
\hline Region & $\begin{array}{c}\text { Outer } \\
\text { Ridius } \\
\mathbf{R}_{\mathrm{o}}(\mathrm{cm}) \\
\end{array}$ & $\begin{array}{l}\text { Thickness } \\
\Delta R(\mathrm{~cm})\end{array}$ & $\begin{array}{l}\text { Cross-Sectional } \\
\text { Anea per Sector } \\
\text { A(cm }\end{array}$ & $\begin{array}{l}\text { Neutron } \\
\text { Heating } \\
\text { J/cmb }\end{array}$ & $\begin{array}{c}\text { Gamma-Ray } \\
\text { Heating } \\
1 / \mathrm{cm}^{6} \\
\end{array}$ & $\begin{array}{c}\text { Gamms-Ray } \\
\text { plus Neutron } \\
\text { Heaaing } \\
\text { J/cmb }\end{array}$ \\
\hline Plasma & 15.00 & 15.00 & & & & \\
\hline Vacuum & 49.97 & 14.97 & & & & \\
\hline $\mathrm{Al}_{2} \mathrm{O}_{3}$ & 50.00 & 0.03 & 9.43 & 1473.0 & 598.0 & 2071.0 \\
\hline Nb & 50.10 & 0.10 & 31.45 & 741.0 & 5767.0 & 65230 \\
\hline Li & 53.10 & 3.00 & 972.64 & 97746.0 & 16208.0 & 113954.0 \\
\hline Nb & 53.20 & 0.10 & 33.43 & $\bowtie 14.0$ & 5953.0 & 6567.0 \\
\hline $\mathrm{Be}$ & 55.70 & 2.50 & 855.30 & 187680.0 & 29359.0 & 217039.0 \\
\hline $\mathrm{Nb}$ & 55.80 & 0.10 & 35.06 & 403.0 & 5634.0 & 6037.0 \\
\hline $\mathrm{t.i}$ & 60.30 & 4.50 & 1641.32 & $104: 40.0$ & 23109.0 & 127249.0 \\
\hline Nb & 60.40 & 0.10 & 37.95 & 313.0 & 5632.0 & 5945.0 \\
\hline $\mathrm{Al}_{2} \mathrm{O}_{3}$ & 60.43 & 0.03 & 11.39 & 572.0 & 611.0 & 1183.0 \\
\hline $\mathrm{Nb}$ & 60.53 & 0.10 & 38.03 & 305.0 & 5586.0 & 5891.0 \\
\hline C & 64.89 & 4.34 & 1709.77 & 60585.0 & 51580.0 & 112165.0 \\
\hline $\mathrm{Nb}$ & 64.97 & 0.10 & 40.82 & 183.0 & 4691.0 & 4874.0 \\
\hline $\mathrm{Al}_{2} \mathrm{O}_{3}$ & 65.00 & 0.03 & 12.25 & 324.0 & 498.0 & 822.0 \\
\hline Nb & 65.10 & 0.10 & 40.90 & 180.0 & 4525.0 & 4705.0 \\
\hline L.i & 67.60 & 2.50 & 1042.22 & 56105.0 & 10178.0 & 66283.0 \\
\hline Nb & 67.70 & 0.10 & 42.54 & 163.0 & 4392.0 & 4555.0 \\
\hline $\mathrm{Al}_{2} \mathrm{O}_{3}$ & 67.73 & 0.03 & 12.77 & 265.0 & 475.0 & 740.0 \\
\hline $\mathrm{Nb}$ & 67.83 & 0.10 & 42.62 & 160.0 & 4343.0 & 4503.0 \\
\hline c & 82.57 & 14.74 & 6964.58 & 76419.0 & 92353.0 & $16 \$ 772.0$ \\
\hline Nb & 82.67 & 0.10 & 51.94 & 39.0 & 1591.0 & 1630.0 \\
\hline$A !_{2} O_{3}$ & 82.70 & 0.03 & 15.59 & 72.0 & 162.0 & 234.0 \\
\hline $\mathrm{Nb}$ & 82.80 & 0.10 & 52.03 & 39.0 & 1502.0 & 1541.0 \\
\hline${ }^{6} \mathrm{Li}$ & 85.20 & 2.40 & 1266.69 & 56531.0 & 2636.0 & 59167.0 \\
\hline $\mathrm{Nb}$ & 85.30 & 0.10 & $\$ 3.60$ & 35.0 & 1386.0 & 1421.0 \\
\hline $\mathrm{Li}$ & 86.30 & 1.00 & 539.10 & 2142.0 & 1220.0 & 3362.0 \\
\hline Nb & 86.40 & 0.10 & 54.29 & 34.0 & 1324. & $\mathrm{~J} 358$. \\
\hline${ }^{6} \mathrm{Li}$ & 88.80 & 2.40 & 1320.97 & 283.0 & 2358.0 & 2641.0 \\
\hline $\mathrm{Nb}$ & 88.90 & 0.10 & 55.86 & 31.0 & 1276.0 & 1307.0 \\
\hline $\mathrm{Al}_{2} \mathrm{O}_{3}$ & 90.90 & 2.00 & 1142.28 & 887.0 & 2052.0 & 2939.0 \\
\hline $\mathrm{Cu}-\mathrm{Al}_{2} \mathrm{O}_{3}$ & 92.90 & 2.00 & 1154.85 & 1840.0 & 15457.0 & 17297.0 \\
\hline $\mathrm{Al}_{2} \mathrm{O}_{3}$ & 94.90 & 2.00 & 1192.55 & 570.0 & 4709.0 & 5279.0 \\
\hline $\mathrm{Cu}-\mathrm{Al}_{2} \mathrm{O}_{3}$ & 134.90 & 40.00 & 28877.52 & 5689.0 & 48788.0 & 54477.0 \\
\hline $\mathrm{Ti}$ & 140.00 & 5.10 & 4404.50 & 19.0 & 230.0 & 249.0 \\
\hline
\end{tabular}

${ }^{a} A=2 \pi R_{0} \Delta R\left(1-\Delta R / 2 R_{0}\right)$ is the total area per turoidal segment. Since $\sim 5.9 \%$ of the area is filled by radial structure, the area per radial sector is obtained by multiplying by $0.94 / 100$.

$b_{\text {Based on } 2.80 \times 10^{\prime \prime}} \mathrm{n} / \mathrm{cm} /$ pulse which leads to $\mathrm{Q}_{\mathrm{N}}=20.53 \mathrm{MeV} / \mathrm{n}$ deposited as recoverable energy or a zotal of $22.30 \mathrm{MeV} / \mathrm{n}$. 


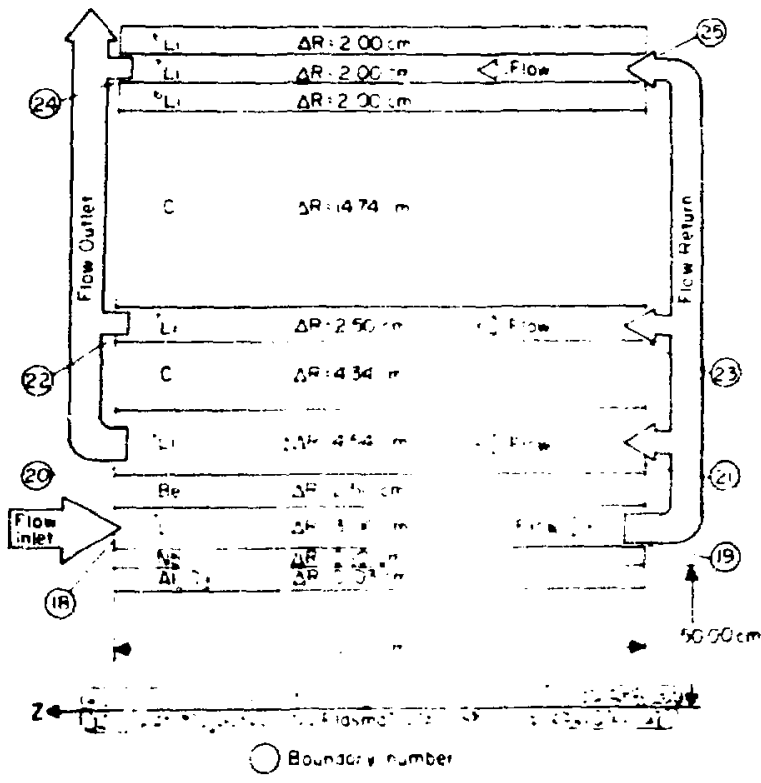

Fig. 4.5-io.

Finite-element geometry and lithium flow pattens used to simulate modular beat transfer.

power cycle; the spatial dependence of the bremsstrablung absorption was obtained from a separate photon transport calculation which interfaced with the time dependence of the plasina temperature; and the spatial dependence of garnma and neutron energy deposition (Figs. 4.5-7 and 4.5-8) was analytically simulated for the solid regions of the blanket. Lithium flow commenced immediately after the 1-sec plasma cooling stage at a rate necessary to yield a coolant hold-up time of $\sim 2$ sec; during this period of flow a Lyon-type relationship was used to determine the Nusselt number $\left(\mathrm{Nu}=h \mathrm{D}_{\mathrm{E}} / \mathrm{k}\right)$ for a given flow channel. Referring to Fig. 4.5-10, lithium enters boundary 18 at a fixed inlet temperature (assumed in the present calculation to be $1100 \mathrm{~K}$ ) and reenters the blanket at boundaries 21,23 , and 25 with an enthalpy corresponding to the instantaneous conditions at boundary 19: in this way the flow manifolding problem was approximated. Table 4.5-8 summarizes important calculational variables and assumptions. The geometry used is close to that described in Table 4.5-7. Although $\mathrm{Al}_{2} \mathrm{O}_{3}$ and niobium interfacial regions were not represented in this geometry (with the exception of the first wall), interfacial energy sources were incorporated into the calculation to simulate the presence of structural materials.
Figure 4.5-11 B gives the time-dependence of the average temperature for the five solid-containing regions for $\tau_{c}=\check{\gamma}_{i}(3.0 \mathrm{sec})$ and Fig. 4.5-11 A gives a similar plot fcr the mixed-measi exit lithium temperature. The $3-\mathrm{sec}$ dut $y^{\prime}$ cycle includes the nominal 0.120 second burn stagt; approximately 20 power cycles are reciaired before th: blanket achieves a thermal steady-state. Figure 4.5-1? gives the steady-state, ternperature (radial) profile at varous times during the power cycle; five axial positions are included on Fig. 4.5-12. Important points to note are:

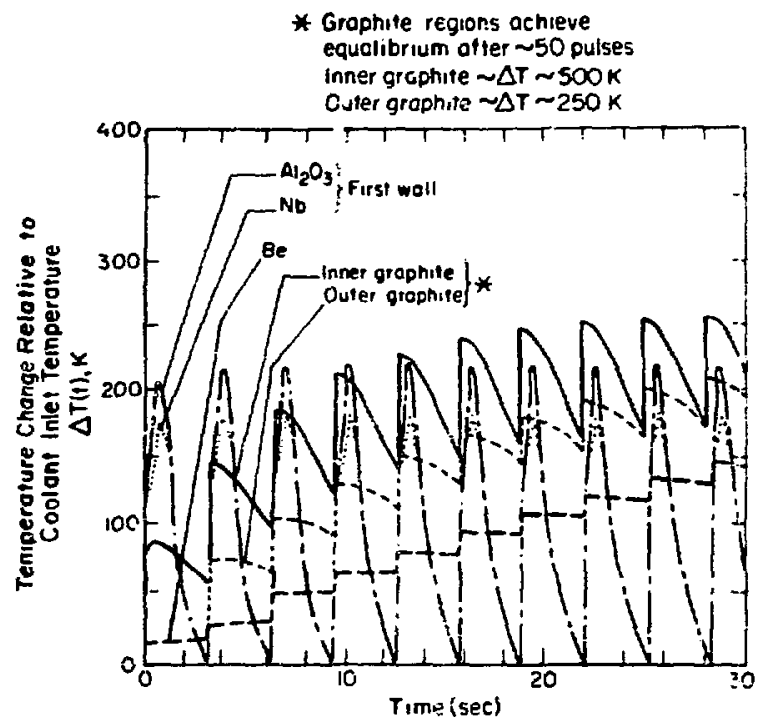

(A)

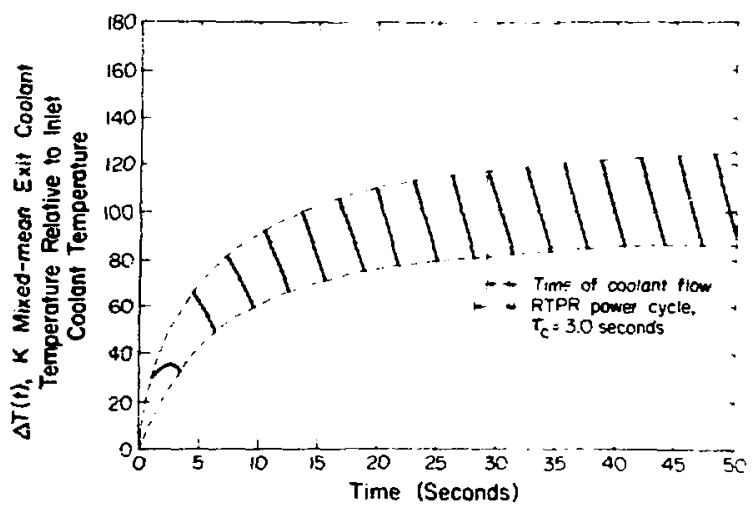

(B)

Fig. 4.5-11.

Time dependence of the average temperature of each blanket region (Fig. 4.5-11) for the case of an initially uniform blanket temperature $(1100 \mathrm{~K})$ and $\tau_{c}=\breve{\tau}_{c}(3.0 \mathrm{sec})$.

A. Solid regions (first wall, $\mathrm{Be}, \mathrm{C}$ ).

B. Mixed-mean exit lithium temperature. 


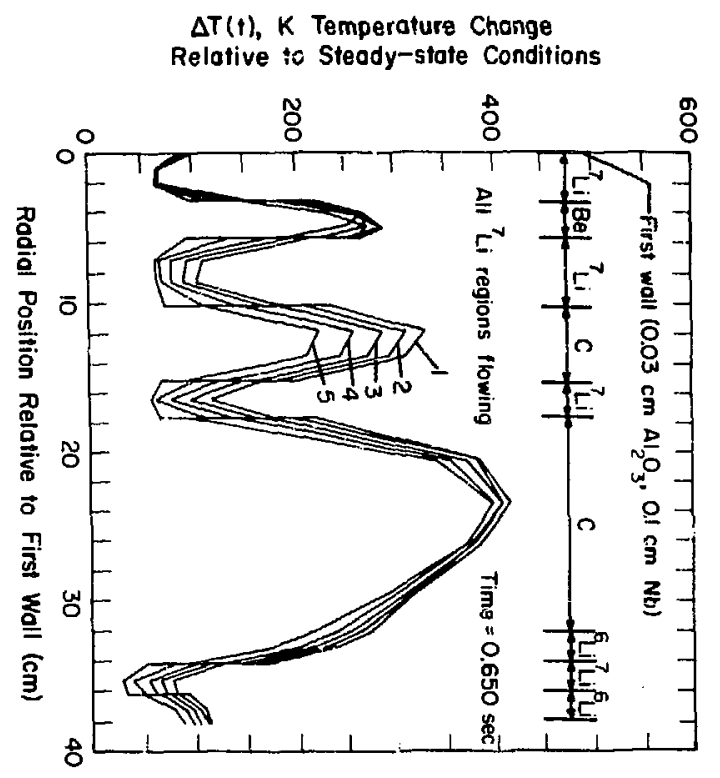

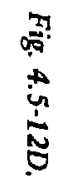

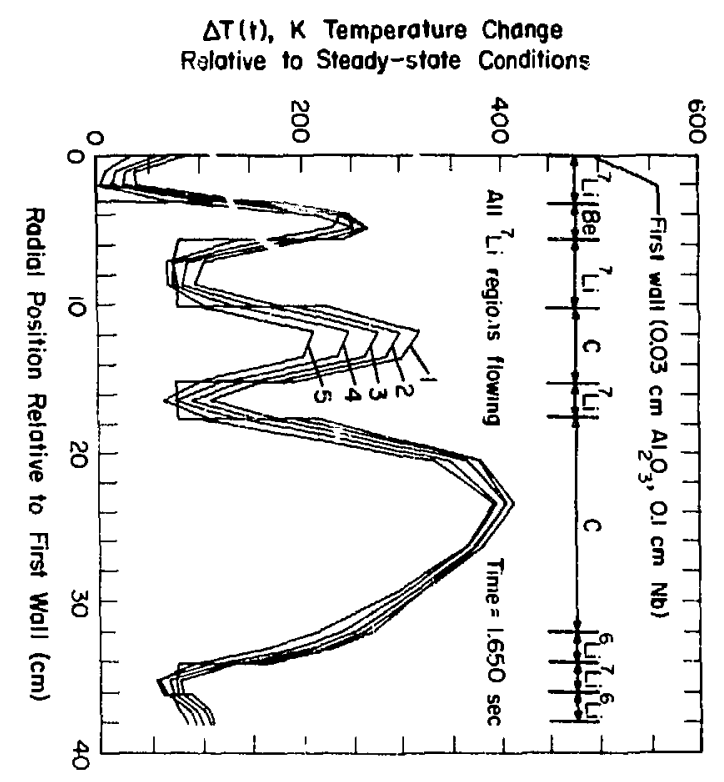

$\frac{\pi}{4}$

$\Delta T(t), K$ Temperature Change Relative to Steady-state Conditions

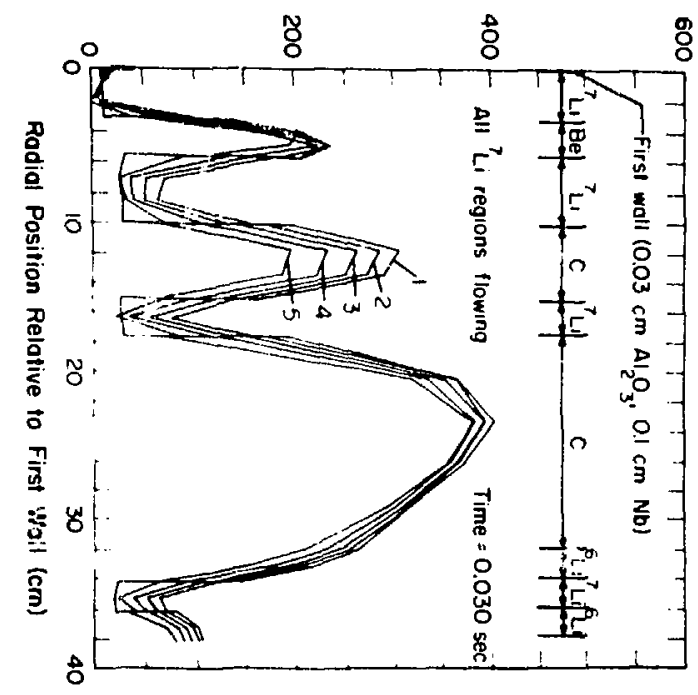

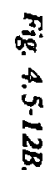

$\Delta T(t), K$ Temperalure Change Relotive to Steady-state Conditions

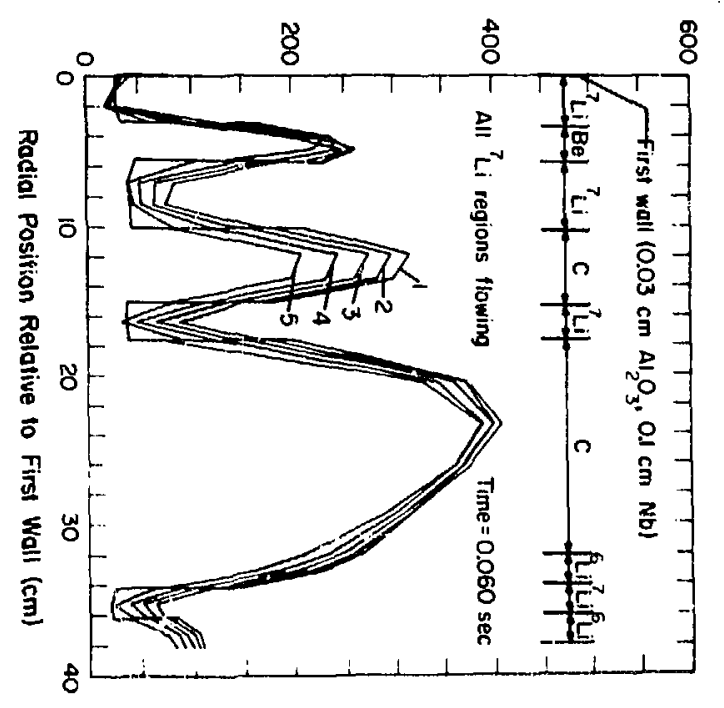


TABLE 4.5-8

\section{IMPORTANT CALCULATIONAL VARIABIES USED FOR RTPR BLANKET HEAT TRANSFER CALCULATION}

\begin{tabular}{|c|c|c|c|c|c|c|c|c|c|c|c|}
\hline \multicolumn{12}{|c|}{ Region } \\
\hline & 1 & 2 & 3 & 4 & 5 & 6 & 7 & 8 & 9 & 10 & 11 \\
\hline Material & $\mathbf{A l}_{2} \mathbf{O}_{3}$ & $\mathbf{N b}$ & $\mathbf{L i}$ & Be & $\mathbf{L i}$ & C & $\mathbf{L i}$ & C & ${ }^{6} \mathrm{Li}$ & $\mathbf{L i}$ & $\mathbf{L i}$ \\
\hline$\mu\left(\mathrm{g} / \mathrm{cm}^{3}\right)$ & 3.80 & 8.55 & 0.43 & 1.80 & 0.43 & 1.80 & 0.43 & 1.80 & 0.43 & 0.43 & 0.43 \\
\hline$C(\mathrm{~J} / \mathrm{g} \mathrm{K})$ & $.4184 / \mathrm{T}^{18}$ & 0.34 & 4.18 & 1.60 & 4.18 & $0.9 ?$ & 4.18 & 0.93 & 4.18 & 4.18 & 4.18 \\
\hline$k(W / \mathrm{cm} \mathrm{k})$ & $309 / \mathrm{T}^{1.2}$ & 0.70 & 0.50 & 0.84 & 0.50 & 0.10 & 0.50 & 0.10 & 4.18 & 0.50 & 4.18 \\
\hline $\begin{array}{l}\text { Ftow }\left(\mathrm{cm}^{3} / \mathrm{sec}\right) \\
\text { Velocity }(\mathrm{cm} / \mathrm{sec})\end{array}$ & & & $\begin{array}{r}3450.0 \\
-364.0\end{array}$ & & $\begin{array}{r}545.0 \\
95.0\end{array}$ & & $\begin{array}{r}984.0 \\
95.0\end{array}$ & & & $\begin{array}{r}010.0 \\
95.0\end{array}$ & \\
\hline
\end{tabular}

\begin{tabular}{l}
\multicolumn{1}{c}{ Parameter } \\
Volumetric Flow \\
Coolant inlet temperature \\
Initial temperature \\
Total energy deposited \\
Duty cycle, $\tau_{C}=\tau_{C}$ \\
Burn time, $\tau_{B}$ \\
Rise time, $\tau_{R}$ \\
Plasma cooling time, $\tau_{Q}$
\end{tabular}

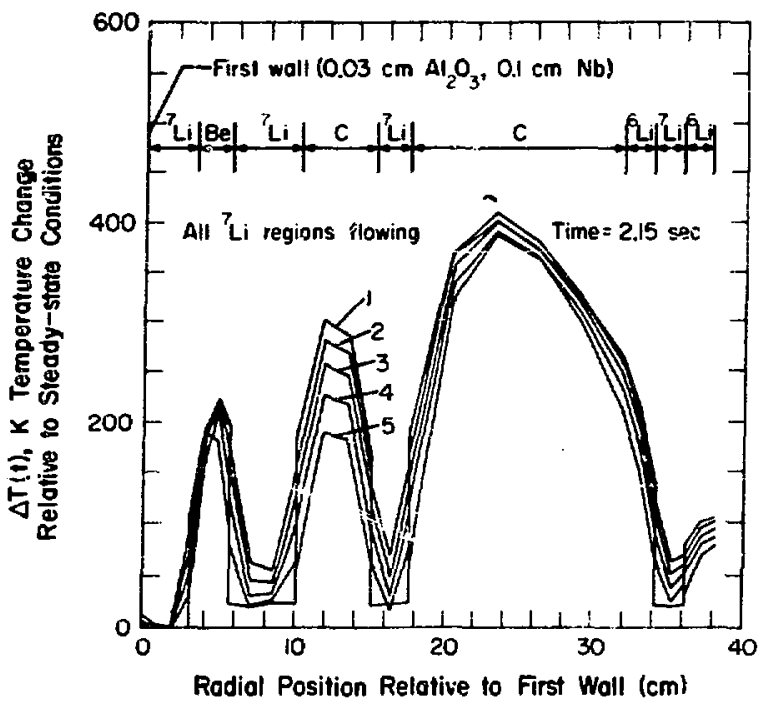

Fig. 4.5-12E.

Fig. 4.5-12.

Radial temperature profile in blanket structure (Fig. 4.5-11) at steady-state for five axial pasitions measured from the inlet of the $2-m$ module, $Z=0.0 \mathrm{~m}$ (1), $Z=0.5 m(2), Z=1.0 m(3), Z=1.5 m(4)$, and $Z=2.0 m(5) . \tau_{c}=\check{r}_{c}(3.0 \mathrm{sec})$.

Valut:
$4200 \mathrm{~cm}^{3} / \mathrm{sec} / 2$ meter segment
$1100 \mathrm{~K}$
$1100 \mathrm{~K}$
$93.1 \mathrm{MJ} / \mathrm{m}$
$3.0 \mathrm{sec}$
$0.07 \mathrm{sec}$
$.031 \mathrm{sec}$
$1.0 \mathrm{sec}$

- The first-wall region (niobium and $\mathrm{Al}_{2} \mathrm{O}_{3}$ ) achieve a steady-state after two to three power cycles.

- The first carbon region experiences the highest temperature $(\sim 350 \mathrm{~K}$ above the inlet coolant temperarure). The beryllium region also experiences an appreciable temperature rise.

- A small but significant amount of heat is generited in the stagnant ${ }^{6} \mathrm{Li}$ region; cooling of this region is needed. - Strong temperature peaking within solid regions of the blanket exist at steady-stace, and accommodation must be made for differential thermal expancion.

These numerical results do not represent optimal conditions, but ar: included solely to illustrate a typical blanket response to the RTPR power cycle. The temperatures reported herein can be varied greatly by appropriate changes in the configuration of coolant channels and solid regions of the blanket, as well as by altering lithium flow rates.

\subsubsection{Effects of Magnetic Field on Stagnant Liquid Metal}

The large, rapidly changing magnetic field has a significant effect on the stationary lithium and the cooling 
ducts. The different interactions which can be identified are:

1. Eddy currents in the lithium and metallic walls of the coolant ducts generated when the magnetic field is time-variant. These currents can give rise to appreciable ohmic loss if they are allowed to occur within large areas of the blanket.

2. Flow resistance and turbulent damping in the lithium coolant because of the coolant-magnetic field interaction lead ro sigaificant reduction in heat transfer coeficients.

3. The lithium can be compressed during the rise and fall of the magnetic field, le $e_{\text {ding }}$ to pressure transients within the blanket.

Ohralc losses in the blanket region have been estimated in Sec. 2.4, and incorporated into the overall RTPR er.ergy balance. The decreased heat transfer incurred by flow stoppage and turbuent damping incurred by the lithium in the presence of the magnetic field have been incorporated into the blanket heat transfer calculations (Sec. 4.4.4 and 4.5.3). Section 4.5.5 estimates the pressure surges which accompany the flow stoppage and estimates tha recovery of flow once the field is removed. The present section addresses the problem posed by item 3 above.

Consider a circular duct of radius $R$ and length $L$, which is oriented with its axis parallel to a magnetic fieid of magnitude B. As indicated in Fig. 4.5-13, a current density; $J$, is established by the azimuthal electric field. This electric field is determined by

$\oint \overrightarrow{\mathbf{E}} \cdot d \vec{l}=-\frac{d}{d t} \iint_{A} \vec{B} \cdot \vec{n} d A$

and the current density is determined by Ohm's law , according to

$\zeta=-\sigma E$

Assuming $\vec{E}$ and $\vec{B}$ are independent of position in the duct, Eq. (4.5-5) is readily integrated, and the result is combined with Eq. $(4.5-i)$ to yield the following dependence of $\overrightarrow{\mathrm{J}}$ on $\mathrm{dB} / \mathrm{dt}$

$J=(\sigma r / 2)(d B / d t)$

The differential force, $d \vec{F}(r)$, exerted on the current

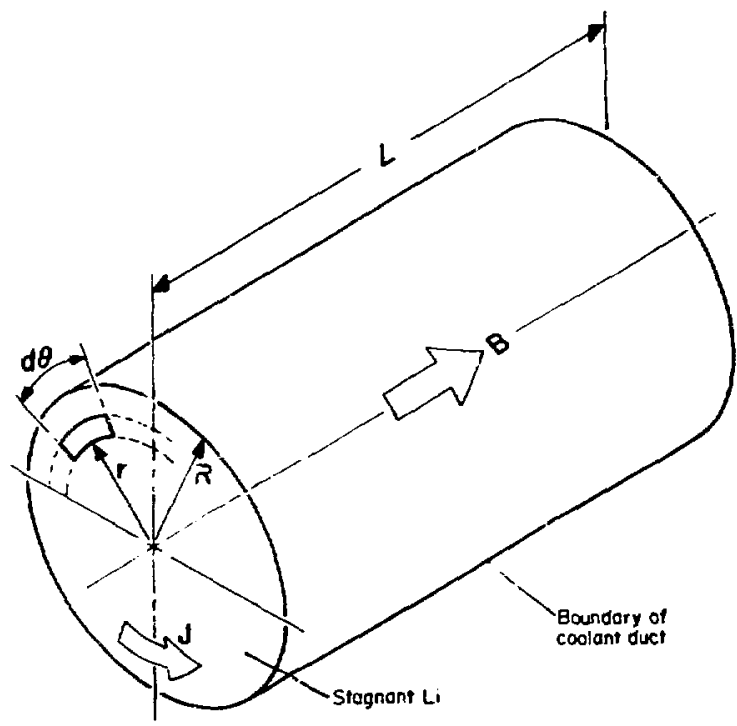

Fig. 4.5-13.

Schematic of geometry used to estimate radial pressure forces acting on a litbium flow channel oriented parallel to a time-varying magnetic field.

elemeni depicted in Fig. 4.5-13 equals $i \overrightarrow{d \vec{l}} \times \vec{B}$. Since the current $i=J L d r$ and $d \ell=r 0$, integration of $d \vec{F}(r)$ around the azimuthal direction results in the following expression for the magnitude of the radial dependence of the induced force.

$$
(d F / d r)=\left(\pi r^{2} L\right) \sigma B(d B / d t)
$$

Equation (4.5-8) is readily integrated to yield the magnitude of the net force acting on the fluid in the radial direction, $F$. The quantity $F$ is expressed as a pressure differential $\Delta P$ between the axis and surface of the idealized duct,

$$
\Delta P=F /(2 \pi R L)=(\sigma / 6) R^{2}\left(d B^{2} / d t\right)
$$

Differentiation of Eq. (2.2-1) gives $B_{0}{ }^{2} \pi / 2 \tau_{R}$ for the maximum of the function $\left(\mathrm{dB}^{2} / \mathrm{dt}\right)$ (neglecting the implosion heating field). For $R \sim 1.0 \mathrm{~cm}, \Delta P$ is evaluated to be $2.76 \mathrm{MPa}$ ( $390 \mathrm{psi}$ ). 
A detailed analysis of this problem is given in Appendix $\mathbf{C}$ for lithium in the coolant channels and in the feed channels; the more detailed analysis is based on a modei which uses rectangular ducts. As shown by the more realistic analysis, pressures on the coolant and coolant ducts are sufficiently high to compress the fluid and perhaps drive coolant out of the ducts in a way analogous to the squeezing of a toothpaste tube. The effect is not believed to be structurally harmful, although fatigue of the coolant duct weldments under this pcriodic flexure cannot be ignored. Special precauxions may be required at the endis of each flow channel as well as at the manifold sidewalls; this magnetically induced pressure will add to the static pressure; pressure differentials across coolant ducts on the order of mega Pascals are to be avoided. The walls of the coolant ducts are placed back-to-back so that the net force on rwo adjacent walls is zero.

The flow patterns established by this magnetic pressure are difficult to understand because of the complex coupling of flow channels and the drgree to which the effect depends on the flow direction (i.c., whether the flow axis is paraliel or perpendiculai to the time -arying magnetic field). A few basic points pertaining to this problem. however, can be made.

1. The magnetic field inhibits flow across field lines. For example, the fluid in the feed lines is estimated to move $\sim 0.020 \mathrm{~mm}$ (see Appendix $\mathrm{C}$ ) if inertial effects are negiected.

2. The magnetic pressure in the flow channel, which tends to cause flow ou: of the channel, is smaller than the counteracting magnetic pressures in the menifold.

Based on this information, the estimated flow patterns indicate that:

1. Some coolant will be lost from the manifold, but the total volume will be considerably less than $1.0 \mathrm{~cm}^{3}$.

2. The fluid loss from the feed line could allow a small amount of fluid to escape from the coolant channel along the inner wall. This loss is expected to be very small in that at this point no magnetic pressure exists to induce this flow (see Appendix C).

3. A slight amount of circulation flow may exist in the flow channel, but the channef should remain full of fluid.

\subsubsection{Lithium Flow Transients within Blanket}

During the power cycle, the quantity of lithium which flows through each $2-\mathrm{m}$ module is $4200 \mathrm{~cm}^{3} / \mathrm{sec}$ averaged over the $\sim 63 \%$ of the RTPR duty cycle during which the compression field is zero. The transient and steady-state flow conditions are calculated for the nlow model depictec in Fig. 4.5-14. The cross sectional areas for the various flow channels are those summarized in Table 4.5-7.

4.5.5.1 Andytical Model. The momentum equation which describes flow across magnetic field lines is given by

$$
\begin{aligned}
\rho(\partial \vec{v} / \partial t & +\vec{v}(\vec{v} \cdot \vec{v}))=-\vec{V} P+\vec{\nabla} \cdot \vec{T}+\vec{J} \times \vec{B} \\
& +\rho_{e} \cdot \vec{E}+\vec{F}_{B} .
\end{aligned}
$$

This general expression is neduced to the one-dimensional case and used to describe the velocity in each of the six coupled flow channels depicted in Fig. 4.5-14. Asstming incompressible flow $(\vec{\nabla} \cdot \vec{v}=0$ ), negiecting body forces $\left(\vec{F}_{g}=0\right)$, sett ng the electric field, $\vec{E}$, to zero, and using the relationship $\vec{\tau}=\vec{n} \vec{v} \vec{v}$. Eq. $(4.5-10)$ becomes

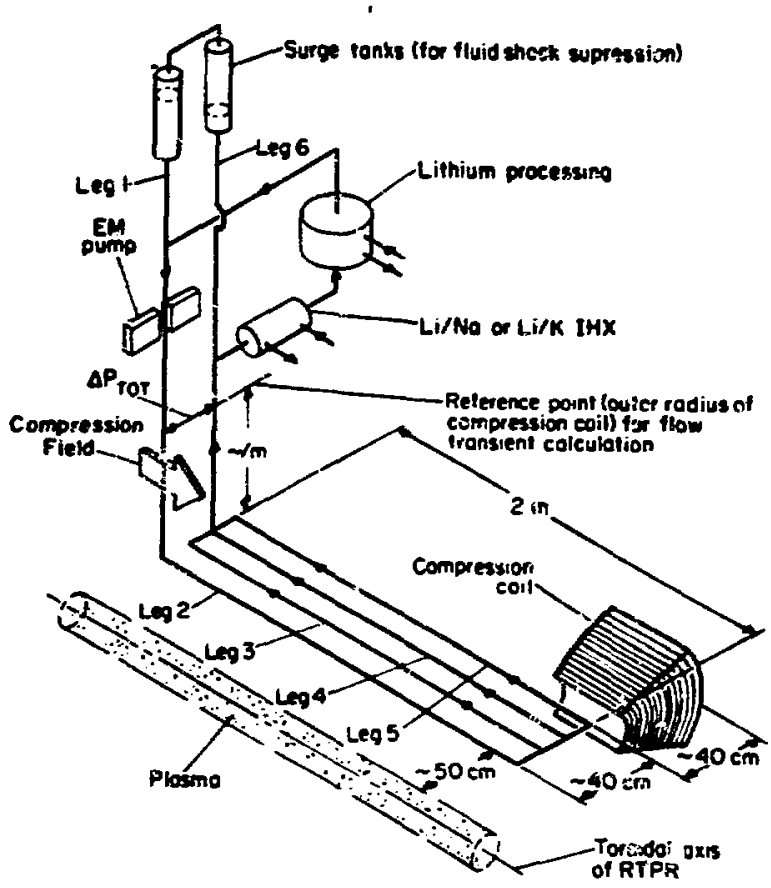

Fig. 4.5-14.

Flow diagram used to describe the transient and steady-state flow conditions in a $2-m$ reactor module. 
$\rho \mathrm{d} \overrightarrow{\mathrm{V}} / \mathrm{dt}=-\vec{\nabla} \cdot \mathrm{P}+\eta \nabla^{2} \overrightarrow{\mathrm{v}}+\overrightarrow{\mathrm{J}} \times \overrightarrow{\mathrm{B}}$

$(4.5-11)$

By substitution of Ohm's law, $\vec{J}=\sigma(\vec{E}+\vec{V} \times \vec{B})$, and for flow in the $x$-direction, the one-dimensional form of Eq. $(4.5 \cdot 1.1)$ becomes

$\rho \partial v_{x} / \partial t=-\partial x+n \partial^{2} v_{x} / \partial y^{2}+\sigma v_{x} B^{2} y$

Defining an average velocity, $(V)=(1 / R) \int_{0}^{R} v_{x} d y$, and introducing the Hartmann number, $H_{a}^{i}=B_{y}^{2} \sigma R^{2} / \eta$, results in

$\partial\langle\mathrm{v}\rangle / \partial \mathrm{t}=-(1 / \rho) \Delta \mathrm{P} / \Delta \mathrm{L}$

$+(f / 4 R)\langle v\rangle^{2}+H_{a}^{2}(T / \rho)\langle v\rangle / R$

where the friction cosficient, $f / 2=(\eta / \rho)$ $\left(\partial v_{x} / \partial y\right)_{y=0} /\left((V)^{2} / 2\right)$ has been introduced, and the length of a given leg is equal to $\Delta \mathrm{L}$. Finally, introduction of the Reynolds's number, $R e=\rho(V) R / \eta$ and a dimensionless ame, $\tau=(\eta / \rho)\left(t / R^{* 2}\right)$, where $R^{*}$ is a characteristic dimenasion of the system depicted on Fig. 4.5-14, the momentum equation for the $i^{\text {th }}$ leg is given by

$$
\begin{aligned}
& d \operatorname{Re}_{1} / \mathrm{d} \tau=-\Delta \mathrm{P}_{1} / \mathrm{P}_{1}+\mathrm{F}_{1} \mathrm{Re}_{1}^{2}+\mathrm{H}_{1} \operatorname{Re}_{1} \\
& P_{1}=(\eta / a)^{2}\left(R_{1} / R^{*}\right)^{2}\left(\rho \Delta L_{1} / R_{1}^{3}\right) \\
& F_{1}=(f / 4)\left(R * / R_{1}\right) 2 \\
& H_{1}=H_{a}^{2}\left(R * / R_{1}\right)^{2} C /(1+C)
\end{aligned}
$$$$
(1=1,6)
$$

The conductivity ratio, $C=2 t_{w} \sigma_{w} / D_{E} \sigma_{L i}$ has been introduced, the duct wall thickness is $\mathrm{t}_{w,}$, the hydraulic diameter of the flow channel is $D_{E}$. and the respective electrical conductivities are $\sigma_{w}$ and $\sigma_{1, i}$. This set of six coupled differential equations was solved numerically, subject to the following conditions of mass continuity and total pressurc balance.

$$
\begin{aligned}
& \operatorname{Re}_{1}(A / R)_{1}=\operatorname{Re}_{2}(A / R)_{2} \\
& \operatorname{Re}_{1}(A / R)_{1}=\operatorname{Re}_{6}(A / R)_{6}
\end{aligned}
$$

$\operatorname{Re}_{1}(A / R)_{1}=\sum_{i=3,5} \operatorname{Re}_{i}\left(A / R_{1}\right)$

$$
{ }^{\Delta P_{T O T}}=\Delta P_{1}+\Delta P_{2}+\Delta P_{3}+\Delta P_{6}
$$

$A_{i}$ is the cross-sictional area of the $i^{\text {th }}$ leg and $\Delta P_{T O T}$ represents the pressure differential across the lithium inlet-outlet as measured at a reference point extemal to the compression field (see Fig. 4.5-14). The quantity $\Delta \mathrm{P}_{\mathrm{TO}}$ is held fixed and in this way the flow situation within the magnetic field is decoupled from the external riping and surge tanks depicted in Fig. 4.5-14. The spatial variation of the manifolds at the juncture of legs 3,4, and 5 has been neglected. The pressure drop resulting from now across magnetic field lines is taken into account only for legs $\mathrm{I}$ and 6 , i.e., $\mathrm{Ha}:=0$ for $i=2$ through $\mathrm{S}$.

4.5.5.2 Resuits and Conclusions. Figure 4.5-15 illustrites the dependerce of mass flow rate on the driving pressure, $\Delta \mathrm{P}_{\text {TOT }}$, for the geometry depicted in Fiz. 4.5-14. The recovery of steady-state fluw conditions is illustrated on Fig. 4.5.16 for various values $\Delta P_{\text {. }}$ CT , and Fig. 4.5-17 shows the effecis of the magnetic field on lithium flow for the design conditions seiected for the RTPR. The flow of lithium is reestablisied within $\mathbf{4 0} \mathbf{m s e c}$, and, as expected, the effects of the compression field on the lithium flow is dramatic. The degree of flow stoppage, however, can oe controlled to some extent by yariation of the conductivity ratio, $c$. The dependence of flow rate on $C$ is indicated on Fig. 4.5-17.

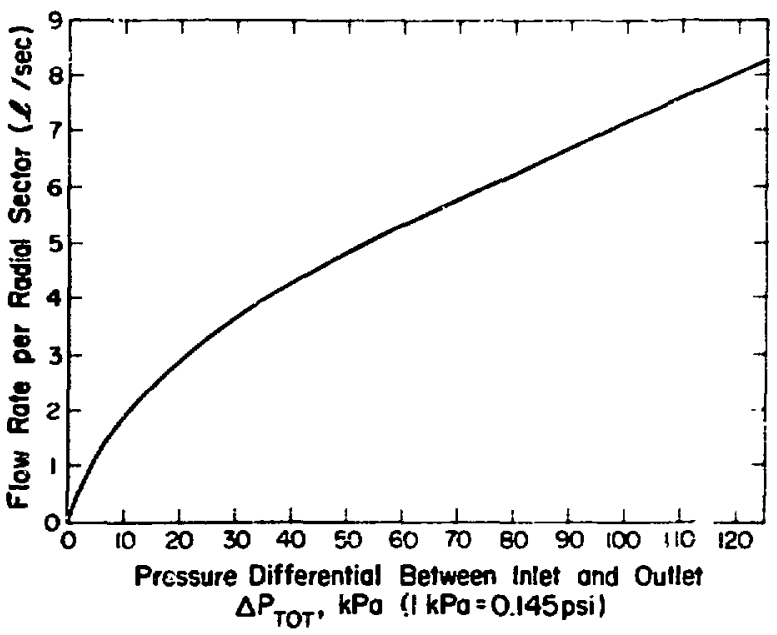

Fig. 4.5-15.

Dependence of flow rate on pressure differential at coolant inlei and outlet. 


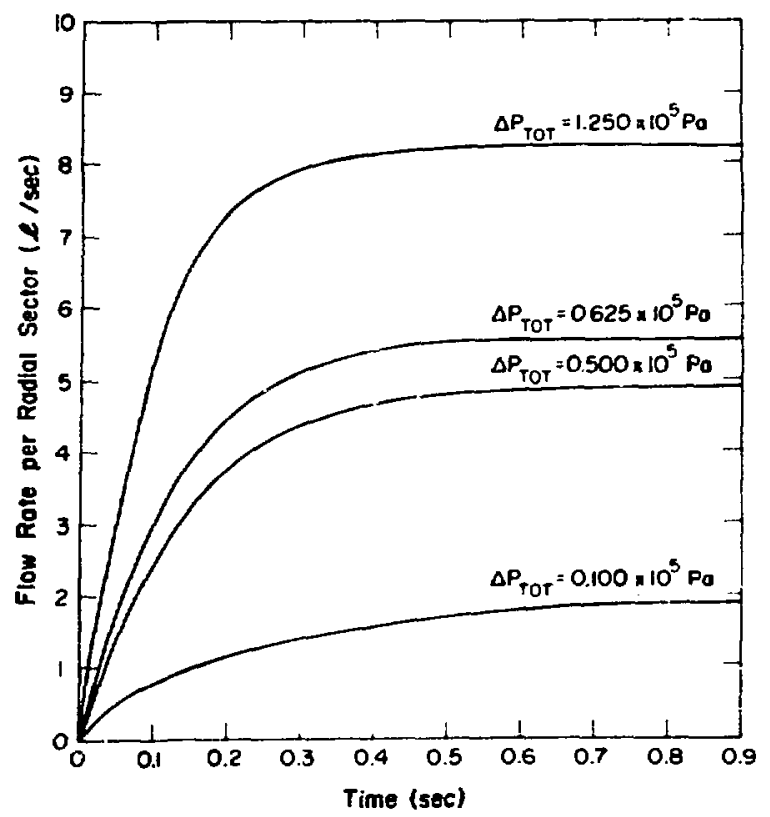

Fig. 4.5.16.

Recovery of steady-state flow conditions for various values of pressure differentials at coolant inlet and outlet.

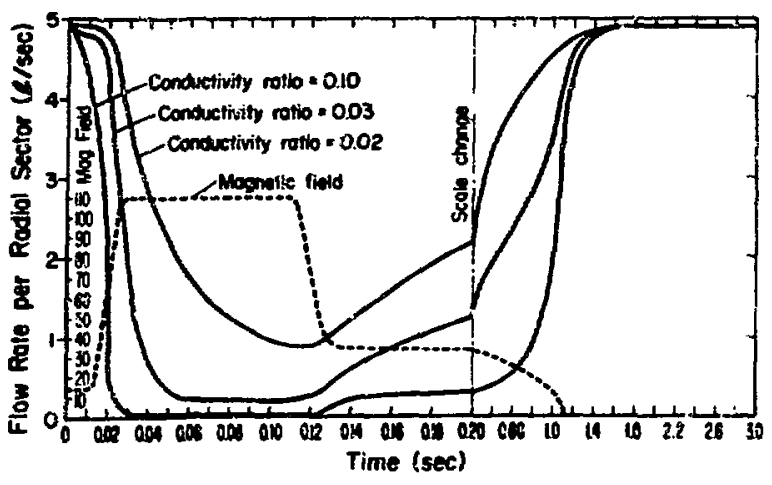

Fig. 4.5-17.

Dependence of litbism coolant flow on magnetic field and conductivity ratio.
4.5.6 Blanket Component Interactions with Forces other than Electromagnetic

The magnetic forces discussed above are the major concerrs on the structural properties of the blanket. As implied, however, the other forces may need to be tailored to mitigate rather than compound the structural problems in the blanket. Forces considered include those resulting from the vacuum-atmosphere interfaces, lithium hydraulic pressures, differential thermal expansions, gravity, etc.

4.5.6.I Forces Related to Pressure Differentials. The entire blanket and compression coils are enclosed in a vacuum tunnel (Chapter 3). The pressurc in this tunnel will arerage about 100 micror:s and for structural purposes may be considered zero. Thus, the lithium channels will experience forces corresponding to the absolute pressure of the lithium coolant. In nearly all cases this pressure force and the gravitational head is opposed by an equal and opposite lithium pressure in an adjacent segment (see Fig. 3.1-1). The inner diameter of the module adjacent to the plasma, the outer diameter of the blanket adjacent to the thermal insulation, and the ends of the modules where the lithium manifolds are adjacent to the vacuum ash removal port represent regions of the blanket which are not subject to this kind of self-reinforcernent. On the inner diamerer of the blanket, the lithium channels are rounded so that the internal pressure is resisted by a hoop stress. With a maxinium static pressure of $3.4 \times 10^{5} \mathrm{~Pa}(50 \mathrm{psi}$ ) expected in the lithium channels, this corresponds to a simple hoop stress of only $2.1 \times 10^{7}$ $\mathrm{Pa}$ (3100 psi) which is very much less than allowable stresses for niobium-zirconium alloy at $\sim 1000 \mathrm{~K}$. A similar situation exists at the lithium manifolds, whish are also exposed on one side to a vacuum. In this case, however, if the wall thickness of niobium is mantained at $0.5 \mathrm{~mm}$, the stress corresponding to the maxirnum size of the manifold (at the outer periphery of the manifold where the transition to tubes is made, see Fig. 3.1-1) is only $3.3 \times 10^{7} \mathrm{~Pa}(5000 \mathrm{psi})$. Although these stress levels appear acceptible and feasible on a steady-state basis, the fatigue aspect of periodic flow surges and pressure evacuations is recognized and must be considered in more detailed blanket designs.

4.5.6.2 Forces Related to Differential Thermal Expansion. From room temperature to an average operating temperature of $\sim 900 \mathrm{~K}$, the blanket will expand about $4 \mathrm{~mm}$ in minor diameter. This expansion is accomedated by the thermal insulation shell surrounding the blanket. Each module expands about $8 \mathrm{~mm}$ in the axial direction, 
and provision for this expansion is made in the vacuum ducts between modules. Additionally, axial xxpan, on is used to reduce neutron streaming between modules by providing some overlap of the lithium manifolds when the core is at operating temperature by allowing clearance when the blanket is cold. By use of the modular concept the 56-m radius torus does not increase in radius as would be the case for a continuous torus. $A$ continuous torus $112 \mathrm{~m}$ in diameter would expand nearly $70 \mathrm{~cm}$, causing considerable problems in mour: ing lithium surts compression coil leads, and anchoring of the blanket. The center of the modules, however, will remain fixed in space, and expansions of connections will he provided from that fixed location.

An unusual thermal expansion problem involves the lithium during the bum portion of the sycle. During this time the lithium nearly instantaneously increases in temperature by $\sim 50 \mathrm{~K}$ (see Fig. 4.5-11B). Also, during this time an intense magnetic field is present which prevents the free expansion of the lithium out of the blanket through the manifold. If this field completely inhibited lithium flow, the pressure inside the lithium channels would increase. However, one can approximate the actual pressure by assuming that the lithium heating is linear with time, that the magnetic field is constant, that the ducts and lithium are imcompressible and that the lithium is able to escape through both inlet and outlet manifolds. In this case, the pressure in the lithium channels parallel to the magnetic field is given by ${ }^{22}$

$$
\Delta P=\frac{1.3 B^{2} \bar{u}}{\pi} C
$$

where the velocity $\vec{u}$ in this expression is calculatid from the total volume expansion of the coolant divided by the area of the manifolds and the time of heating.The quantities $C$ and $n$ are, respectively, the conductivity ratio and the viscosity. ${ }^{22}$ This expression yields $\Delta P \sim 2.5 \times 10^{3} \mathrm{~Pa}$ (0.4 psi). which is negligible. Hence, depending on the conditions of flow across magnetic field lines, the pressures generated by the expansion of instantaneously heated lithium may or may no: be a problem

\subsubsection{Blanket Penetrations}

Some effort has been expended in the blanket design to prevent excessive neution streaming through inhomogeneities in the blinket. All streaming effects have not been negated in the blanket cross section shown in Fig. 3.2-2). Generaliy, streaming between segments is not expected to be severe because the segments are forced together by various of the aforementioned forces, e.g., thermal expansion and internal fluid pressures in the blanket segments. Furthermore, no gross inhomogeneity in the theta direction of the blanket cxists. A possible problem between segments caused by a straight-line path through the electrical insulator was eliminated by twisting segments just slightly:

In the circumferential direction around the major torus diameter, however, severe streaming problems are encountered. Blanket penetrations are necessary for fueling, cooling, instrumentation, and ash removal. Radiation streaming through fueling passthroughs is not a significant problem because the fuel is injected by means of moderately high-pressure, small-diameter $(1 \mathrm{~mm})$ tubing. The cooling manifolds represent a source of streaming, since lithium is only a fair neutron deflector, although only a small percentage of the torus periphery is composed of this uniform material.

The "ash" removal system requires a major penetration into the plasma zone through the blanket. In the current design, the duty cycle is limited by the allowable duct size for ash removal. Because this duct size represents a controlling factor for the reactor duty cycle (and therefore its capital costs) some effort was made to maximize the exhaust duct area at the expense of limited neutron streaming. In subsequent design iterations, this design feature needs to be intensively studied and other designs, perhaps involving shorter modules, will be investigated.

\section{REFERENCES}

1. S. C. Bumett, W. R. Ellis, T. A. Oliphant, and F. L. Ribe, "A Reference Theta Pinch Reactor (RTPR): A Study of a Pulsed High-Beta Fusion Reactor Based on the Theta Pinch," Los Alamos Scientific Laboratory Report LA-5121-MS (December 1972).

2. G. 1. Bell, W. H. Borkenhagen, and F. L. Ribe, "Feasibility Studies of Pulsed, High- $\beta$ Fusion Reactors," in Proc. of the B.N.E.S. Conf. on Nucl. Fusion Reactors, UKAEA Culhzm Laboratory, Sept. 17-19, 1969, P. 242.

3. F. L. Ribe, T. A. Oliphant, Jr., and W. E. Quinn, “Feasibility Study of a Pulsed Thermonuciear Reactor," Los Alamos Scientific Laboratory Report LA-3294-MS (Mzy 1965).

4. George I. Bell, "Neutron Blanket Calculations for Thermonuclear Reactors," Los Alamos Scientific Laboratory Report LA-3385-MS (September 1965).

5. George I. Bell, "Neutron Blanket Calculations for Thermonuclear Reactors, II," Los Alamos Scientific Laboratory Report LA-41 31-MS (April 1969).

6. G. I. Bell, Los Alamos Scientific Laboratory, personal communieation, February 1972. 
7. K. D. Lathrop, "DTF-IV, A FORTRAN-IV Progtam for Solving the Multigroup Transport Equation with Anisotropic Scattering," Los Alamos Scientific Laboratory Report LA-337 3 (November 1965).

8. D. W. Muir, Los Alamos Scientific Laboratory, personal communication, June 1972.

9. D. W. Kusner and R. A. Dannels, "ETOG-1, A FORTRAN-IV Program to Process Data from the ENDF/B File to the ME.FT, GAM, and ANISN Formats," Westinghouse Report WCAP-3845-1 (1969).

10. Donald J. Dudziak, "Neutronic Analysis of CTR Blanket Benchmark by Discrete Ordinates," Trars. Am. Nucl. Sec. 15(2), 630 (1972).

11. M. A. Abdou and C. W. Maynard, "Neutron Source Geometry Effects on Fusion Reactor Blankets," Trans. Am. Nucl. Soc. 15(1), 34 (1972).

12. D. W. Muir and M. E. Wyman, “A Tritium-Production Measurement with Application to Fusion Reactor Blanket Design," Trans. Am. Nucli. Soc. 15(2), 631 (1972).

13. M. E. Wyman, "An Integral Experiment to Measure the Tritium Production from ' $\mathrm{Li}$ by $14 \mathrm{MeV}$ Neutrons in a Lithium Deuteride Sphere," Los Alamos Scientific Laboratory Report LA-2234 Rev. (1972).

14. Don Steiner, Oak Ridge National Laboratory, personal ermmunication, Aligust 1972.

15. W. F. Vogelsang, "Breeding Ratio, Inventory, and Doubling Time in a D-T Reactor," Trans. Am. Nucl, Soc, 15(1), 33 (1972).

16. J. J. Ritts, M. Solomito, and D. Steiner, "Kerma Factors and Secondary Gamma-Ray Sources for Some Elements of Interest in Thermonuclear Blanket Assemblies," Oak Ridge National Laboratory Report ORNL-TM-2564 (1970).

17. Donald J. Dudziak (Ed.), "ENDF Formats and Procedures for Photon Production and Interaction Data," Los Alamos Scientific Laboratory Report LA-4549 (July 1971).

18. Donald J. Dudziak, Robert E. Seamon, and Dante V. Susco, "LAPHANO: A P $P_{0}$ Multignoup Photon Production Matrix and Source Code for ENDF," Los Alamos Scientific Laboratory Report LA-47 50-MS (Janiary 1972).

19. K. D. Lathrop, "GAMLEG: A FORTRAN Code to Prociuce Multigroup Cross Sections for Photon Transport Calculations," Los Alamos Scientific Laboratory Report LA-3267 (April 1967).

20. D. Steiner, "The Nuclear Performance of Fusion Reactor Blankets," Nucl. Appl, and Tech. 9,83 (1970).

21. G. L. Kulcinski, University of Wisconsin, personal communication, July 1972.

22. J. R. Moszynski, M. Petrick, T. A. Coultas, "Cooling of Controlled Thermonuclear Fusion Reactors of Toroidal Configurations," Texas Symposium on the Tecbnology of CTR, Univ. of Texas, Austin, Texss, NOv. 1972.

\subsection{BIOLOGICAL SHIELDING SYSTEM*}

\subsubsection{System Requirements}

The biological shielding system is required to reduce the radiation levels of neutrons and photons to no more than the Maximum Permissible Exposure (MPE) as recommended by the National Council on Radiation Protection and Measurements (NCRP) in both controlled and nomcontrolled areas of the plant.

A preliminary assessment of the biological shielding requirements has been made to visualize the plant engineering layout and to identify interface constraints between subsystems. The shielding design concept reflects needs related to assembling, operating, and maintaining the reactor system. The toroidally segmented reactor allows for some ease in managing in each of these phases.

\subsubsection{General Shielding Engineering Licyout}

4.6.2.1 Reactor Core Components. One criterion for the shielding system design is that it accommodates the transfer of radioactive toroidal segments or subsections with minimal dependence on mobile casks and simplifies supplementary shielding for remote and direct manual handling of the segmented reactor components. This scheme also allows the Magnetic Energy Transfer and Storage System (METS) to be located close to the reactor core system.

The biological shielding consists prinarily of a thick concrete containment structure and tunnel system immediately adjacent to the reactor proper (see Fig. 3.1-1). Within this containment, remote controls will be provided for assembly or disassembly, limited maintenance, transfer to storage, and for managing maintenance areas (hot cells). The radioactive and nonradioactive components will be transferred to and from the hot cells or the reactor equipment buildings by a network of tracked tunnels.

4.6.2.2 Controlled and Noncontrolled Areas. Depending on the thickness and composition of the shielding structure, the areas adjacent to the structure can be classed as controlled or noncontrolled. The NCRP defines a controlled area as one that requires control of access, or:cupancy, and working conditions for radiation protection purposes, i.e., the exposure of persons to radiation is

D. J. Dudziak, LASL, S. A. W. Gerstl, P. j. Persiani, ANL. 
under supervision of a radiation protection supervisor. The Maximum Permissible Exposure (MPE) recommended - by the NCRP for controlled areas is $2.5 \mathrm{mrem} / \mathrm{hr}$ for neutron and photon radiacion. In the noncontrolled (free access) areas, the MPE recommendation is $0.25 \mathrm{mrem} / \mathrm{hr}$.

Immediately adjacent to the shielding structure are the METS, the shock coil capacitor banks, the vacuum pumping system, the fuel processing system, and the heat exchangers, EM pumps, and lithium purification and dump tanks. It is assumed that in some of these areas, particularly the METS system and capacitor banks, a need may arise for daily maintenance or continuous access; for this case the approach adopted for shielding is that these be classified as noncontrolled areas.

\subsubsection{Shield Design and Composition}

Neutrons. The estimate of neutron sticlding requirements is based on experience gained in correlating the analytical method of the combination removal-diffusion thcory ${ }^{1}$ with experimental results for shielding of fast reactor systems. Tiie thickness of the main concrete shielding structure is approximately $2 \mathrm{~m}$ in the region near the reactor core. The neutron attenuazion in the concrete of $20 \mathrm{~cm}$.'decade of reduction effects a reduction factor for the neutron flux (fast and slow) of approximately $10^{10}$ for an MPE of $0.25 \mathrm{mrem} / \mathrm{hr}$.

Preliminary numerical computations were developed to estimate a conservative lower limit for neutron and gamma-ray shielding requirements. The distributions of neutron flux, neutron dose rate, and neutron heat generation rate in the biological shield were calculated with the ANISN code ${ }^{2}$ using a one-dimensional $S_{6}$ transport approximation with 100 group $P_{3}$ cross sections. The neutron spectrum and intensity as calculated for the blanket system (Sec. 4.5.2) was taken as an angular boundary source at a point inside the compressior coil region (racius of $132 \mathrm{~cm}$ ). The resulting neutron fluss distributions are shown in Fig. 4.6-1, where the total flux of neutrons with energies above $1 \mathrm{MeV}$, above $0.4 \mathrm{eV}$, and the thermal flux are plotted. A total neutron flux level of 0.43 neutrons $/ \mathrm{cm}^{2} \mathrm{sec}$ is obtained at the outer surface of the biological concrete shield, of which most are of thermal energies. These computations show a neutron attenuation of one decade per $\sim 15 \mathrm{~cm}$, resulting in a total neutron flux reduction factor of $\sim 10^{-13}$. The resulting neuiron dose rate at positions within the concrete is plotted in Fig. 4.6-2. The neutron component of the total dose rate at the outer surface of this biological shield is $9.0 \times 10^{5} \mathrm{mrem} / \mathrm{hr}$, which is well below the maximum permissible level of $0.25 \mathrm{mrem} / \mathrm{hr}$ for an uncontrolled area.

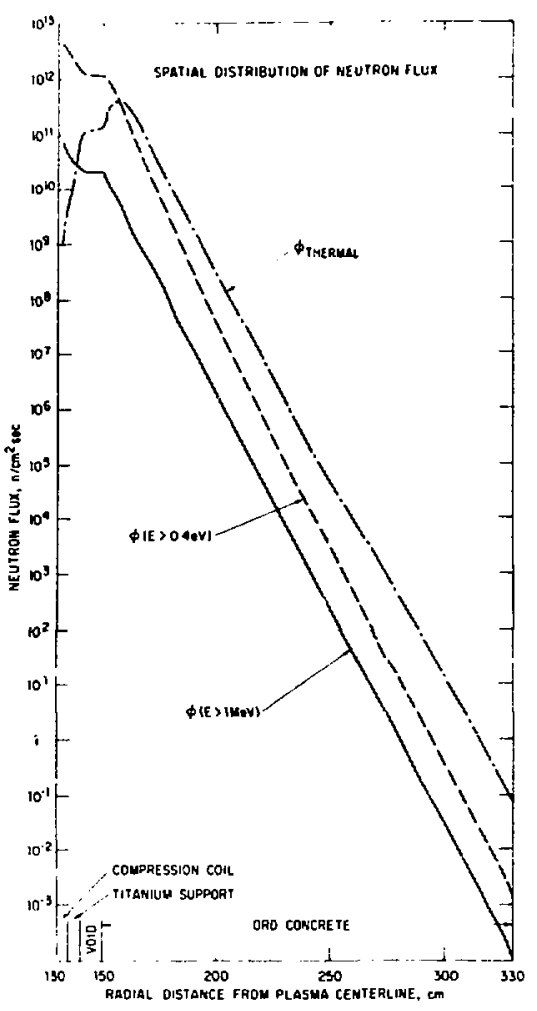

Fig. 4.6.1.

Neutron fluxes $\phi\left(E_{T H}\right), \phi(E>0.4 \mathrm{eV})$ and $\phi(E>1$ $\mathrm{MeV})$ as a function of tbickness of concrete outside the compression coil.

The segmented design of the reactor core may not allow complete control of neutron streaning between the modules. To minimize these effects, the shielding in these regions can be supplemented by selective and localized use of the high- $Z$ material and borated graphite inserted into the main shieldin $y$ structure. The effects of radiation streaming are not included in this preliminary evaluatior. Because of this exclusion and the fact that an isotropic source approximation was assumed for the present calculations, it is recommended that the results presented in Figs. $4.6-1$ and $4.6-2$ be considered as a lower limit of a conservative shield design.

Photons. The distribution of photons and the attendant radiation heating in the biological shield were calculated with the ANISN code ${ }^{2}$ using an $\mathbf{S}_{\mathbf{8}}$ transport approximation with 21-group $P_{3}$ cross sections. Gamma rays generated in the regions of the implosion coil, 


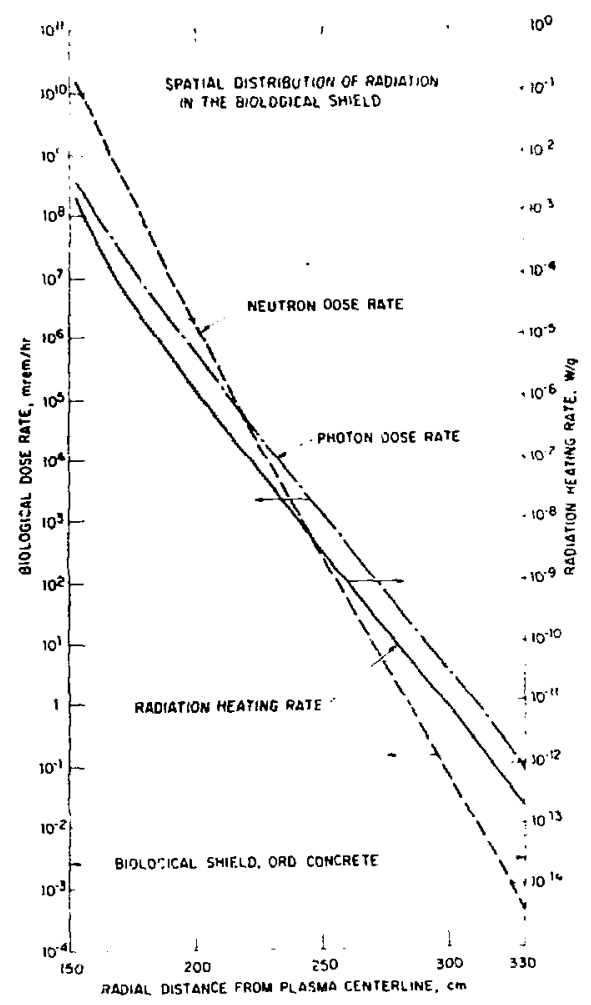

Fig. 4.6.2.

Biological dose rate and neutron and gamma beating as a function of concrete thickness outside the compression coil.

compression coil, titanium structure, and thermal insulations were taken from the blanket calculations in Sec. 4.5.2 and treated as isotropic volumetric gamma-ray sources. The component of the dose rate generated inside the biological snield by these photons is included in Fig. 4.6-2. The gamma dose rate at the outer surface of the ordinary concrete is $0.024 \mathrm{mrem} / \mathrm{hr}$, which is higher than the neutron dose rate at that position and appears to be the controlling component. This result should also be considered a lower limit of the photon dose rate since the photon production within the concrete itself was neglected in this preliminary evaluation. Adding the gamma and neu tron dose rates gives a total biological dose rate of about $0.024 \mathrm{mrem} / \mathrm{hr}$ immediately behind the concrete shield, which is still a factor of 10 smaller than the mum permissible exposure in uncontrolled areas.

The use of a derse tarytes concrete $\left(4.5 \mathrm{gm} / \mathrm{cm}^{3}\right)$ mixture represents an allowable option, since its attenuation is almost twice as effective as ordinary concrete. The use of heavy concrete ys ordinary concrete, however, should be balanced against cost and space considerations.

Other shielding options such as steel or lead liners within the concrete will be effective in terms of cost and space requirements, particularly with respect to minimizing the distance between the reactor core and capacitor energy storage banks.

Heating of the Shielding Structures. Heat generation rates arising from the interaction of neutrons and gamma rays with the shielding material were estimated from the calculated neutron and photon distributions within the concrete shield. The spatial distribution of the total neutron and photon energy deposition in the ordinary concrete shielding structure is included in Fig. 4.6-2. Maximum heat generation rate in the concrete appears at the inner surface of the shielding structure and is estimated to be $0.57 \times 10^{-3} \mathrm{~W} / \mathrm{g}$. This radiation heating together with the resulting temperature gradient in the biological shield serve as the basis for determining cooling requirements for the shield structure. Preliminary estimates indicate that cooling ducts provided only at the interior surface of the biological shielding will provide more than adequate cooling.

Use of heavy concrete or the insertion of metal liners is suggested. However, the introduction of steel or heavy element pellets uniformly distributed in the concrece aggregate or insertion of steel plates would probably require an auxiliary conling systemi as a result of additional heating introduced by neutron captures and subsequent gamna-ray energy deposition. No attempt to estinate the temperature rise or the temperature gtadient in the heavy concrete system has been made. In usirg the heavy concrete the photor attenuation is primarily affected. The thickness of the $i$ ain shielding system is still determined by the gamma radiation and therefore is still retained at $2 \mathrm{~m}$.

4.6.2.4 Shielding of Coolant System. The lithium coolant is expected to contain dissolved niobium introduced as corrosion and sputter products. Section 4.5.2.4 considers the analysis of the rate at which this kind of activity ingresses into the lithium. Volume II gives the expecied time dependence of the resulting ${ }^{93 \mathrm{~m}} \mathrm{Nb}$ and ${ }^{92} \mathrm{Nb}$ activity. The specific activity influencing the shielding requirements of the coolant conduit system is the isomeric sta:e ${ }^{92 \mathrm{~m}} \mathrm{Nb}$ with gamma-ray energies of $1.82 \mathrm{MeV}(32 \%)$, and $0.93,0.9 \mathrm{MeV}(68 \%)$ in caseade. The half-value layers for attenuating this radiation in barytes concrete are approximately 3 and $2.4 \mathrm{~cm}$, respectively. On this basis it is estimated that a thickness of $75 \mathrm{~cm}$ of barytes concrete shielding ducts is required to atrenuate the gamma dose to the noncontrolled permissible level of $0.25 \mathrm{mrem} / \mathrm{hr}$ in 
the adjacent areas. If the niobium content in the coolant is considerably reduced by lithium clean-up systems, then consideration must be given to the neutron-induced radioactive impurities present in the lithium coolant system.

A preliminary estimate of the photon radiation intensity resulting from impurities within the lithium is obtained by assuming that the major contributors result from the reactions: ${ }^{23} \mathrm{Na}(\mathrm{n}, 2 \mathrm{n}){ }^{22} \mathrm{Na},{ }^{23} \mathrm{Na}(\mathrm{n}, \gamma){ }^{24} \mathrm{Na}$, ${ }^{54} \mathrm{Fe}(\mathrm{n}, \gamma){ }^{55} \mathrm{Fe}$, and ${ }^{54} \mathrm{Fe}(\mathrm{n}, \alpha){ }^{51} \mathrm{Cr}$. The daughter products of the above isotopes emit high-energy gamma rays. The elements were assumed to be impurities in the lithium coolant with soncentrations in the range of $100 \mathrm{ppm}$ and have been summarized in Table 4.5-5.

It is estimated that a $75-\mathrm{cm}$ thickness of ordinary concrete shielding ducts is required to attenuate the gamma flux to a noncontrolled MPE level in the adjacent areas. An attempt is made to include effects from other impurities, with gamma active daughter products arising mainly from the $(n, \gamma),(n, \alpha),(n, p)$, and $(n, 2 n)$ reactions.

Secondary sodium $(n, p)$ activity in the intermediate heat exchanger $1 \mathrm{HX}$ is assumed tc he lo: 3 ted immediately behind the biological shield wall and is calculated to be $0.33 \times 10^{-15} \mathrm{Ci} / \mathrm{cc}$, which is well below the secondary sodium activity allowed in the LMFBR IHX (approximately $6.0 \times 10^{-11} \mathrm{Ci} / \mathrm{cc}$ ).

A more definitive design study will allow identification and assessment of a broader range of related radiological shielding problems. The coolant clean-up systems, such as the liquid metal-molten salt contactors and hot and cold traps represent localized sources of radiation and will probably require special shielding. A more extensive study would identify and assess more pertinent daughterproduct schemes for all possible nuclear reactions and all isotopes that may influence the other plant shielding design.

\section{REFERENCES}

1. J. Butler and A. F. Avery, "Removal-Diffusion Theory," in Engineering Compendium on Radiation Sbielding, R. G. Jaeger, Ed., v. 1 (Springer-Verlag, New York, 1968) pp. 273-301.

2. W. W. Engle, "A Users Manual for ANISN: A One Dimensional Discrete Ordirsite Transport Code with Anisotropic Scattering," Union Carbide Corp., Nuclear Div. Report K1693 (1967).

\subsection{FUEL SUPPLY AND REMOVAL SYSTEM *}

\subsubsection{System Requirements}

This sysiem is required to supply a 50:50 DT mixture at the pressure required for the burn stage of the reactor

D. C. Hess, ANL. cycle and to remove the helium, ash, and other reaction products along with unburned DT. The rate of ash removal is limited by surface area available for vacuum ducting and constitutes a constraint on the rate at which the RTPR can be pulsed.

\subsubsection{Physical Principles}

The fueling and flushing process can be described by four basic steps. First, it is necessary to have a proper amount of fuel in the plasma chamber at the start of implosion heating to protect the plasma from contamination. 'Second, it is necessary that sufficient cooling gas be introduced during the D-T burn (near point $b$, Fig. 2.2-1) to protect the plasma from further contamination. Third, it is necessary to supply the gas at or near the end of the burn (point d, Fig. 2.2-1) to transfer the plasma energy to the wall in a controlled manner. Finally, it is necessary to remove is much of the helium ash and other contaminating products as possible to prepare for introduction of a fresh fuel charge for the next cycle.

Because of the amount of gas involved and the limited area available for penetration of the blanket by vacuum ducts, the reduction of helium ash by decreasing the pressure to a low base value is not practical. Instead, the pressure will be maintained approximately constant, and the helium percentage will be lowered by dilution of the mixture with extra fuel.

To facilitate the calculations we assumed that, during the initial implosion-heating stage, $100 \%$ ionization and trapping by the implosion field will occur so that "snowplow" action of the field wili effectively remove all of the gas from the region outside the plasma. Furthermore, the region between the first wall and the plasma is assumed to be a pure vacuum. Any error in this assumption will have negligible effect on the calculated flow and pumping rates, but lack of isolation of the plasma from the cold neutral gas and the possibility of turbulent conditions in the cool gas may have adverse effects on the stability of the plasma. ${ }^{2}$

To simplify the model, the D-T burn computations do not account for the existence of energetic neutral particles such as have been observed outside plasmas of lesser density surrounded by a nomina! vacuum. ${ }^{3 A}$ These particles presumbably originate principally in the outer regions of the plasma and are in fact sometimes used for diagnosis in plasme experiments.

Since the production of energetic particles and photons begins as soon as the plasma begins to burn, it would be desirable to have gas surrounding the plasma as soon as possible to protect the plasma from heavy particles which may be emitted from the walls. However, the presence of such a cool gas outside the plasma vill 
increase the cooling of the plasma. It will probably be necessary to allow the plasma to reach temperatures of the order of 6-10 keV prior to introduction of the cooling gas. At these temperatures the plasma cooling resulting from bremsstrahlung and conduction through the cooling gas will not exceed the fusion energy release in the plasma. These ideas, nowever, will have to be tested by more detailed bum computations which account explicitly for conductive energy losses. For purposes of calculation it has been assumed that a density $\left(1.0 \times 10^{14} \mathrm{~cm}^{-3}\right)$ which is suitable for the later cooling stage ( $? \mathrm{sec}$ cooling stage) will be attained in $30 \mathrm{msec}$ after the implosion heating cycle begins. Alternative arrangements would include (1) extending this time to the end of the D-T burn or (2) introduction of the gas later in le cycle at a nonuniform rate; the rate of inflow depends on the time allowed for the development of the desired pressure.

After a brief equilibration during passage through the blanket walls, the cooling gas will be at a temperature of $810 \mathrm{~K}$ for purposes of both removal and supply. Hence, the pressure of $17 \mathrm{mT}$ Trr at $300 \mathrm{~K}$ has been increased proportionally to $46 \mathrm{mT}$ Torr at $810 \mathrm{~K}$. All calculations are made for this temperature.

The limitation on pumping for this system is set by the conductance of the duct penetrating the blanket; the amount of penetration is limited by factors such as shielding, cooling, and electrical load requirements. At the pressures involved in this system the flow is viscous, so the conductance of the ducts depends on the viscosity. Estimates have been made on the basis of tabulated viscosities with suitable correction for the molecular weight, ${ }^{5}$ and these values have been used to correct the constant in the formulas used for conductance calculations (the formulas are normally for air at $273 \mathrm{~K}$ or $298 \mathrm{~K})$.

All calculations are made for a 2-m-long module. Fueling is assumed to take place at the center of each module, through a small $(\sim 1 \mathrm{~mm})$ tube which penetrates the lithium duct to the first wall. The high-pressure (atmospheric or greater) gas requires a trivial flow area compared to the low-pressure exhaust system. The timing accuracy of the supply valve system need not be better than about $1 \mathrm{msec}$. In general the calculations are for an operating reactor and are not concerned with starrup or shutdown conditions. Requirements and assumptions inherent to this analysis are shown in Table 4.7.1.

\subsubsection{Design of Fueling System}

As discussed above, it is desirable in the proposed design to establish the gas blanket quickly by a large, short-time pulse of gas during the burn time (between $b$ and $c$ in Fig. 2.2-1). For a density of $1.4 \times 10^{14} \mathrm{~cm}^{-3}, a$
TABLE 4.7-1

\section{SUMMARY OF ASSUMPTIONS}

1. The region outside the plasma during implosion heating can be taken as a good vacuum.

2. A gas blanket will protect the plasma from most contaminants originating at the wall.

3. The total burn and cooling time is 1 sec.

4. A fuel gas pressure of $46 \mathrm{mT}$ Torr at $810 \mathrm{~K}$ must be present at the start of the implosion heating phase of each cycle.

5. The helium content in the fuel gas must be below some level $(\sim 1 \%)$. Therefore, a suitable amount of diluen, gas must be handled by the system.

6. Gas may be required outside the plasma for plasma protection at some time during the burn and will be required at the end of the burn for plasma cooing.

7. The gas introduced inte the chamber from outside is pure $D-T$ in a 50:50 atomic ratio.

volume of $1500 \ell$ and lithium temperature (fuel will come to thermal equilibrium with the lithium through which the small fuel duct passes) of $810 \mathrm{~K}$, the required flow rate is about 600 Torr-liter/sec. This flow is considerably larger than the capacity of the vacuum puinps; the chamber pressure rises without valving of $t$ the pumps. This flow is maintained for $30 \mathrm{msec}$ and then reduced to a flow of about 19 Torr-liter/sec by partially closing a valve. The reduced gas flow is continued throughout the remainder of the cycle. The temperature of $810 \mathrm{~K}$ corresponds to the more difficult pumping case for the lowtemperature power plant and is used here. This is because the chamber pressure is only $12 \mathrm{mTor}$ for the required gas blanket density rather than about $17 \mathrm{mT}$ orr for the high-temperature power plant case.

Figure 4.7-1 illustrates the time dependence of the chamber pressure. At a time corresponding to point $e$ in Fig. 2.2-1 ( 1 in Fig. 4.7-1) the pressure rises to about $46 \mathrm{mT}$ Torr because of the sudden total release of the magnetic field restraining the plasma pressure. Several possible flow rate-pumping speed combinations are used to attain pressures of $46 \mathrm{mT}$ Trr and $12 \mathrm{mT}$ Trr. Dilution factors for flush times of $2,2.5$, and $3 \mathrm{sec}$ and the residual 


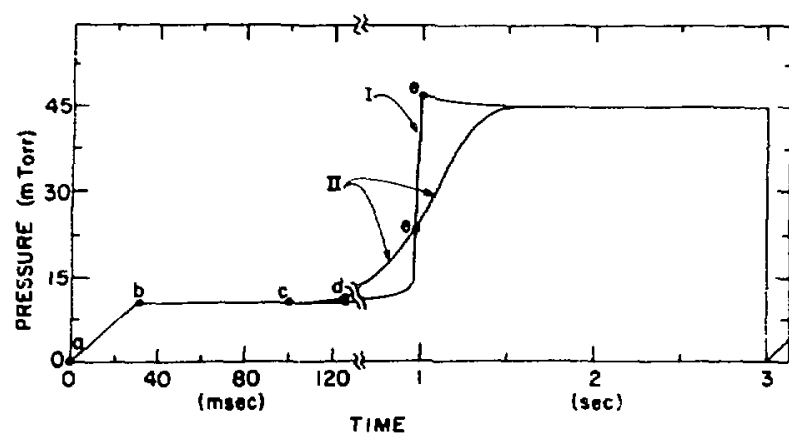

Fig. 4.7-1.

Time variation of neutral gas pressure in plasma chamber during the refueling and plasma cooling stages.

helium concentration in percent for these times are summarized in Table 4.7-2.

If the "boundary" dissolves slowly, the pressure will have some indeterminate value (II, Fig. 4.7-1), but with the flow rate and pumping speed chosen the pressure will fall or rise to the desired value of $46 \mathrm{mTorr}$ before $3 \mathrm{sec}$ has elapsed ( $2 \mathrm{sec}$ after point $\mathrm{e}$ ) in Fig. 2.2-1. After the quench is accomplished, it is assumed that the neutral gas and the gas from the plasma will mix without formation of shock fronts that might have the effect of a piston and reduce the mixing rate and hence the residual helium. The succeeding implosion can occur at any desired later time. Since it has been assumed that the plasma is isolated from the neutral gas until time e in Fig. 2.2-1, gas flowing through the system before this will have no effect on reducing the helium in the plasma.

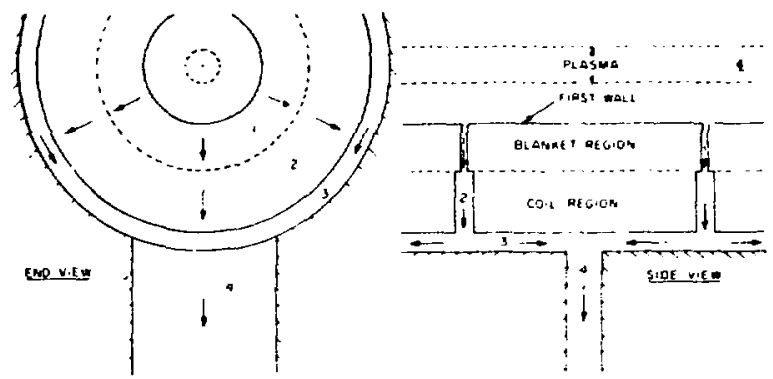

Fig. 4. 7-2.

Diagram of pumping ducts required for each 2-m module.

\subsubsection{Design of the Exhaust System}

Certain aspects of the fueling and exhaust system are prescribed by other features of the system, e.g., the choice of the flow rate at the start of implosion heating is detcrmined by the conditions imposed on the establishment of a protective atmosphere. In other cases some flexibility may be possible. The flow rate required to establish the fuel change permits compromise between pumping speed (pump power and size) and the allowable residual helium for the following cycle. If the residual helium is to be reduced to $1 / n$ of the maximum value at the end of the D-T burn and perfect mixing occurs, it is necessary that an amount of gas equal to $n$ times the fuei charge be mixed with the cooled plasma and that this excess be removed by the pumps. Since the amount of

TABLE $4.7-2$

\begin{tabular}{|c|c|c|c|c|c|c|c|c|}
\hline \multirow[t]{2}{*}{$\underline{S(\ell / \text { sec })}$} & \multicolumn{2}{|c|}{$Q(\ell /$ Torr $/ \mathrm{sec})$} & \multicolumn{2}{|c|}{ Flush Time $=2 \mathrm{sec}$} & \multicolumn{2}{|c|}{ Flush Time $=2.5 \mathrm{sec}$} & \multicolumn{2}{|c|}{ Flush Time $=3.0 \mathrm{sec}$} \\
\hline & $P=46 \mathrm{mTorr}$ & $\mathrm{P}=12 \mathrm{mTorr}$ & $\begin{array}{l}\text { Dilution } \\
\text { Factor }\end{array}$ & $\begin{array}{c}\text { \% Residual } \\
\mathrm{He}\end{array}$ & $\begin{array}{l}\text { Dilution } \\
\text { Factor }\end{array}$ & $\begin{array}{c}\text { \% Residual } \\
\text { He }\end{array}$ & $\begin{array}{c}\text { Dilution } \\
\text { Factor }\end{array}$ & $\begin{array}{c}\text { \% Residual } \\
\mathrm{He}\end{array}$ \\
\hline$\infty$ & 290 & 19.8 & 8.1 & $0.60 \%$ & 10.1 & $0.48 \%$ & 12.1 & $0.40 \%$ \\
\hline 25,000 & 270 & 19.7 & 7.5 & $0.61 \%$ & 9.4 & $0.51 \%$ & 11.3 & $0.43 \%$ \\
\hline 14,000 & 250 & 19.5 & 6.9 & $0.69 \%$ & 8.7 & $0.55 \%$ & 10.4 & $0.40 \%$ \\
\hline 10,000 & 220 & 19.3 & 6.1 & $0.79 \%$ & 7.6 & $0.63 \%$ & 9.2 & $0.52 \%$ \\
\hline
\end{tabular}


fuel in a module at the start of implosion heating is fixed by the plasma requirements, the only variable available to control the dilution is the anount of flush gas. It should be noted that $0.8 \%$ helium will increase the bremsstrahlung loss by about $5 \%$.

The duct for ash evacuation is shown schematically in Fig. 4.7-2. The gas in the plasma chamber must exit through an annulus about $5 \mathrm{~cm}$ wide every two meters (region 1 in Fig. 4.7-2). This annulus is the space between adjacent 2-m-long modules (see Fig. 3.2-1), and extends from the first-wall radius of $50 \mathrm{~cm}$ to the implosionheating-coil radius of $90 \mathrm{~cm}$. At this point the annular duct widens to about $15 \mathrm{~cm}$ as the gas flows between compression coils (region 2 in Fig. 4.7-2). The steel-lined biological shield then forms a collection manifold (region 3 in Fig. 4.7-2) and the gas is finally ducted to the pumps by a 30 by $120 \mathrm{~cm}$ by $600 \mathrm{~cm}$ long duct (region 4 in Fig. 4.7-2) through the concrete shie!d.

The conductance for viscous flow of air at $298 \mathrm{~K}$ for a rectangular duct of height $a$, width $b$, and length $\ell$ is $^{6}$

$\mathrm{C}=0.26 \mathrm{y} \mathrm{a}^{2} \mathrm{~b}^{2} \ddot{\mathrm{p}} \ell$ (Iiters/sec)

where $\mathrm{Y}$ is a factor depending on the ratio $a / b,{ }^{6}$ dimensions are in $\mathrm{cm}$, and $\overline{\mathrm{p}}$ is the average of the inlet and outlet pressures, $\mathrm{p}_{i}$ and $\mathrm{p}_{\mathrm{o}}$, in Torr.

The constant 0.26 involves the gas viscosity and for a 50:50 D-T mixture becomes $16.3 \mathrm{~T}^{\mathbf{0 . 6 7 8}}$. For D-T at a temperature of $T$,

$c=16.3 \mathrm{Y} \mathrm{T}^{-0.687} \mathrm{a}^{2} \mathrm{~b}^{2} \overline{\mathrm{p}} / \mathrm{l}$ (1iters/sec)

If it is assumed that $a / b<0.1, Y$ is proporticnal to $a / b$, and $\mathrm{Y} \approx 2.3 \mathrm{a} / \mathrm{b}$.

For the more realistic case of annular disks, and $a / b<0.1$, a simple integration gives

$c=235 \mathrm{~T}^{-0.678} \mathrm{a}^{3} \overline{\mathrm{p}} / \mathrm{ln}\left(\mathrm{r}_{0} / \mathrm{r}_{1}\right)$

where $a$ is the thickness of the disk, $r_{i}$ is the inner radius, and $r_{0}$ is the outer radius. By analogy with the usual end corrections for short ducts, the inner radius of the 5 -cm-thick duct is taken as $45 \mathrm{~cm}$ instead of 50. The radia! conductance of the $15-\mathrm{cm}$-thick annulus is so much greater than that of the thinner one that no end correction is used.
The pumping system consists of a number of conductances, $C_{i}$, in series. For steady flow it can be shown that with an initial pressure of $p_{1}$ (mTorr), a pumping speed at the pump of $\mathrm{S}$ (liters/sec), and a flow rate of $\mathrm{Q}(\mathrm{mT}$ orrliters/sec).

$\left(p_{I}\right)^{2}=Q^{2} / s^{2}+2 Q \sum_{1=1}^{n}\left(\bar{p} / c_{1}\right)$

The maximum gas flow will occur with an infinite pumping speed,

$$
Q_{M}=\left(p_{1}\right)^{2}\left[2 \sum_{i=1}^{n}\left(\bar{p} / c_{1}\right)\right]
$$

Referring to Fig. 4.7-2 for identification of regions, conductances used for the present calculations were $C_{1}=450 \overline{\mathrm{p}}, C_{2}=20,900 \overline{\mathrm{p}}, C_{3}=1,200 \overline{\mathrm{p}}$, and $C_{4}=1880 \vec{p} \cdot C_{3}$ was calculated as two rectangular ducts with $\ell$ approximately the mean semiperimeter and $b=200 \mathrm{~cm}$, and $\mathrm{a}=15 \mathrm{~cm}$.

Table 4.7-2 shows several possible flow rates, the required pumping speeds, the dilution factors and residual helium for 2-, 2.5-, and 3-sec flush periods.

A pumping speed of 10,000 liters/sec could be obtained by an enlarged version of a Roots blower. Using a volumetric scaling. the pump would be about $140 \mathrm{~cm}$ high, $140 \mathrm{~cm}$ wide, and $: 20 \mathrm{~cm}$ long. The power required would be about $56 \mathrm{~kW}$ per 2-m segment of torus. The backing pump required by a Roors blower would probably be a little smaller, with comparable fower requirements. It could be placed below the Roots pump so that the assembly would fit within the available area. The total power per module would be about $0.1 \mathrm{MW}$. This may not be needed for normal operation in that the work to corapress the gas in the Roots pump will be less than the design value which is for a pressure differential of about 40 Torr, while in operation the differential shoulu be less than 3 Torr. The backing pump, however, will probably be needed for start-up.

If a pumping speed of 25,000 liters/sec is desusd to attain the greater helium reduction in a shorter time, by scaling one finds the Roots pump would be abou: 190 by 190 by $430 \mathrm{~cm}$ with a power requirement of avi $100 \mathrm{~kW}$. The piston pump would have similarly increase 1 dimensions and power requirements. A compromise musi be made between pumping power needs and allowable belium contamination of the fuel. 


\subsubsection{Leakage Through the Walls of the Toroid}

The proposed mechod of assembly of the toroid modules from many smaller segments may allow some leakage to occur unless the pieces are sealed together. However, by comparison with the large gap which occu.s for every $2-m$ segment, this leak age is not a serious fraction of the gas flow through the system. In any case the leak is to the duct formed by the metal shield liner mentioned earlier, and therefore is in parallel with the 5 and $\mathbf{1 5} \mathrm{cm}$ disks.

\section{REFERENCE\&}

1. B. Lehnerc, "Screening of a High-Density Plasma from Neutral Gas Penetration," Nucicar Fusion 8, 173 (1968).

2. D. F. Duchs, H. P. Furth, and P. H. Rutherford. "A Computer Model of the ST Tokamak at High Density," Princeton Plasma Physies Laboratory Report TM-265 (January 1973).

3. F. H. Coensgen, W. F. Cummins, W. E. Nexsen, Jr., and T. C. Simonen, "Initial Operation of the 2XII Experiment," Lawrence Livermore Laboratory Report UCRL-51208 (March 1972).

4. J. R. Hiskes, "Charge Exchange Processes in 2XII," Lawrence Livermore Laboratory Report UCRL-51247 (June 1972).

5. S. Dushman and J. M. Lafferty. Scientifir Foundations of Vacuum Technigue, J. Wiley and Sons, Inc., N. Y. (1965).

6. A. Guthrie and R. K. Wakerling, Vacuum Equipment and Tecbniques (McGraw-Hill Book Company. New York, 1949), Chap. 1.

\subsection{FUEL-ASH PROCESSING SYSTEM*}

\subsubsection{System Requirements}

This system processes the fuel-ash stream leaving the plasma chamber to remove the helium ash and other contaminants and to prepare the remaining fue: for reinjection to the chamber. The total flow consists of upwards of $10 \mathrm{~kg} / \mathrm{hr}$ of gas with a nominal molar composition of $49 \%$ deuterium, $49 \%$ tritium, $\sim 1 \%$ helium $\left({ }^{4} \mathrm{He}\right)$, $<1 \%$ protium, and trace contaminants. The gas leaves the vacuum pumping system (see Sec. 4.7) at a mean temperarace of $\sim 300 \mathrm{~K}$ and a pressure of a few Torr. Following this exit condition, the gas must be compressed, cooled to some extent (depending on the purification process

'L. J. Anastasia, V. A. Maroni, ANL. selected), and enough protium and helium must be separated from the mixture to make it sufficiently pure for reinjection.

\subsubsection{Hedods to separate Protium and Helium}

The plausible approaches considered for the separation of protium and helium included cryogenic distillation, mechanically pumped permeable (metal) membranes, gas chromatography, and supersonic jer separation. Considerable experience exists with regard to large scale separation of hydrogen isotopes by cryogenic distillation, $\mathbf{i}^{17}$ hence, this approach was selected for the RTPR. Isotope separators employing permeable metal membranes represent an equally attractive ${ }^{8,9}$ but less-well-established method, which certainly merits further enginec ring analysis in terms of its applicability to fusion derrices. Processes based on chromatography ${ }^{10}$ or supersonic jer separation ${ }^{11}$ would be impractical (compared to distillation or permeation) for handling the quantities of gas mixture anticipated in the RTPR. This judgment, however, is based solely on a cursory examination of the potential of these methods.

\subsubsection{Design Description of Crymgenic Distillation System}

In the design of the cryogenic distillation system for the RTPR, we considered the operation of a basic unit that could handle $3 \mathbf{k g} / \mathrm{hr}$ of gas; a number of these units could be connected in a parallel circuit capable of processing the entire flow. A flow plan for the unit is shown in Fig. 4.8-1. Gas coming from the vacuum pumps is preconditioned to ambient temperature and pressure and introduced to the equilibrator-heat exchanger (shown as EQ/HEX on the ieft side of Fig. 4.8-1). The mixture is equilibrated at $50 \mathrm{~K}$ to produce the species levels indicated to the right of the EQ/HEX in Fig. 4.8-1. It is then subjected to two stages of compression, Joule-Thomson expansion, and distillation. To improve the separability of protium and deuterium, a catalyzer has been included as suggested by Barron. ${ }^{5}$ Judicious application of heat exchangers throughout the unit (likely locations are indicated on the plan) will be essential to minimize the requirements for heat dissipation and cryogenics.

Calculations of the equilibrium concentrations of the various hydrogen-bearing species in the equilibrator and in the distillation columns were based on data given elsewhere ${ }^{12,13}$ in the literature. Each distillation column contains 60 plates spaced $6.3 \mathrm{~cm}(2.5 \mathrm{in})$ apart. Column 1 is $15.2 \mathrm{~cm}$ ( $6 \mathrm{in})$ : 2 diameter and Column 2 is $7.6 \mathrm{~cm}$ (3 in) 


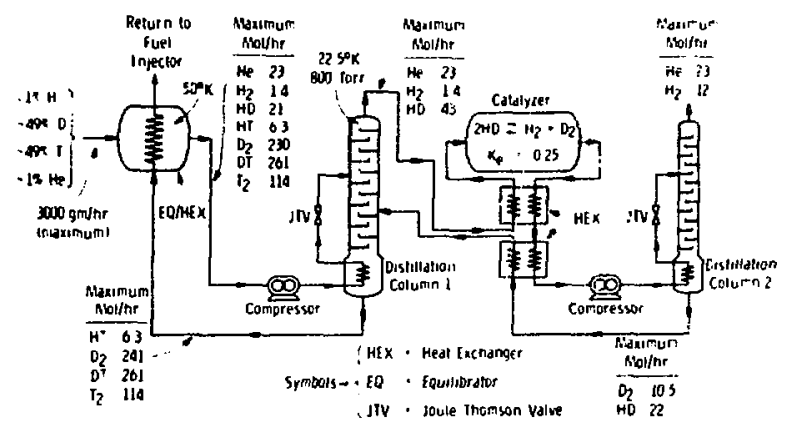

woles

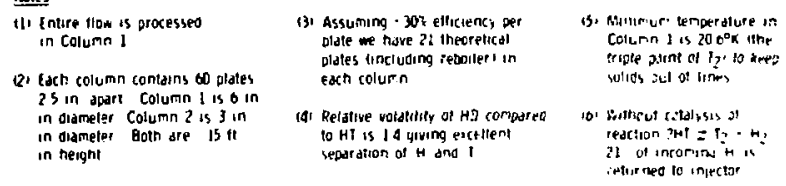

Fig. 4.8-1.

Flow diagram for cryogenic distillation unit proposed for protium and belium separation from fuelash gases.

in diameter; both columns are $\sim 5 \mathrm{~m}$ tall. Thermal insulation adds about $0.6 \mathrm{~m}$ to each diameter. Assuming a plate efficiency of $\sim 30 \%$, the columns have an equivalent of 21 theoretical plates, including one for the reboiler. The relative volatility of $\mathrm{HD}$ compared to $\mathrm{HT}$ is 1.4 , giving excellent separation of $H$ and $T$ in Column 1. With this design, a minimum of $79 \%$ of the incoming protium and all of the helium are separated from the stream and leave the unit at the top of Column 2. Because the protiumhelium effluent probably contains traces of tritium, it cannot be rejected from the plant without prior processing. One possibility is to process this effluent together with the helium purge stream.

The design considerations presented in this section, although preliminary, do indicate that cryogenic distillation is a reasonable method for processing the fuel-ash stream of the RTPR. Aside from the distillation columns, the equipment required for the system is quite small. Preliminary calculations indicate that the heat exchangerequilibrator at the left of Fig. 4.8-1 can be shell-and-tube type, about $13 \mathrm{~cm}(5 \mathrm{in})$ in diameter and $30 \mathrm{~cm}(1 \mathrm{ft})$ long. It requires twenty $1.3 \mathrm{~cm}(1 / 2$-in) o.d. stainless steel tubes without fins to cool the incoming mixture to $50 \mathrm{~K}$ on the shell side. The first compressor requires about $9 \mathrm{~kW}(12 \mathrm{HP}$ ) based on 50\% efficiency. The heat exchangers in tandem with the catalyzer and second column are about $25 \%$ of the size of the one described above. The second-stage compressor requires only fractional $\mathbf{k W}$ power, and the cryogenic heat gain in the cold gas discharge is estimated to be about $100 \mathrm{~W}$. Other heating sources can be made arbitrarily small (of the order of $100 \mathrm{w}$ ), and hence it is estimated that a $3.8 \mathrm{~kW}(5-\mathrm{HP})$ liquefier would make up for all anticipated heat gains.

Since at least three units of the type shown in Fig. 4.8-1 will be required for fuel processing, and perhaps two more for helium-purge gas processing, it will probably take upwards of $60 \mathrm{~kW}(80 \mathrm{HP})$ to operate all of the distillation equipment. This represents a trivial contribution to the circulating power requirements. A room $15 \mathrm{~m}$ by $30 \mathrm{~m}$ with a $10 \mathrm{~m}$ ceiling would probably suffice to house the entire distillation system.

\section{REFERENCES}

1. T. M. Flynn, “Pilot Plant Data for Hydrogen Isotope Distillation," Chemical Engineering Progress 56, 37 (1960).

2. T. M. Flynn, "Studies of the Low-Temperature Distillation of Hydrogen Isotopes," in Advances in Cryogenic Engineering. K. D. Timmerhaus, Ed. (Plenum Press, New York, 1961) p. 236.

3. K. D. Timmerhaus, D. H. Weitzel, and T. M. Flynn, "LowTemperature Distillation of Hydrogen Isotopes," Chemical Engineering Progress 54, 35 (1958).

4. M. P. Malkov et al., "The Separation of Deuterium on an Industrial Scale by Low-Temperature Distillation," in Progress in Cryogenics, Vol. 2, K. Mendelssohn, Ed. (Academic Press, New York, 1960) pp. 71-86.

5. R. Barron, Cryogenic Systems (McGraw-Hill, New York, 1966) pp. 261-263.

6. E. Kanda et al., "Experimental Research on the Rectification of Liquid Hydrogen to Obtain Deuterium," Proc. 2nd U. N. Conf on Peaceful Uses of Atomic Energy, 4, 550 (1958).

7. M. P. Malkov et al., "Industrial Separation of Deuterium by Low-Temperature Distillation," Proc. 2nd U. N. Conf. on Peaceful Uses of Atomic Energy, 4, 491 (1958).

8. E. H. Kobisk, Oak Ridge National Laboratory, private communication (1973).

9. H. Brodowski, H. Gibmeier and E. Wicke, “H/D Separation Factors Using Pd and Pd/Ag Membranes." Z, Physik. Chem., $49,222(1966)$.

10. D. L. West and A. L. Marston, "Gas Chromatographic Separation of the Hydrogen Isotopes," J. Am. Chem. Soc. 86, 4731 (1964).

11. H. Mikami, "Separation of a Three-Component Gas Mixture in an Axisymmetric Supersonic Jet," 1 \& EC Fundamentals 9. $121(1970)$.

12. R. Viallard, in Nouveau Traite de Cbimie Minerale, Vol. 1,P. Pascal, Ed. (Masson et Cie, Paris, 1956) p. 924.

13. H. M. Mittelhauser and G. Thodos, "Vapor Pressure Relationships of Hydrogen, Deuterium, Tritium, and their Diatomic Combinations," Cryogenics, 4368 (December 1964). 


\subsection{LITHIUM PROCESSING SYSTEM*}

\subsubsection{System Requirements}

The lithium processing system recovers the tritium bred in the lithium; maintains the lowest possible inventory of tritium in the lithium during normal operation; and provides for the monitoring, control, and removal of all impurities which have compromising effects on materials compatibility and the overall blanket neutronics. The methods selected to accomplish these design objectives are considered in order below.

\subsubsection{Methods of Recovering Tritium from Lithium}

4.9.2.1 The Behavior of Tritium in Liquid Metals. Measurements of equilibrium hydrogen pressures as a function of hydrogen content will be imperative for the final selection of a tritium recovery scheme. Figure 4.9-1 is a typical family of pressure-composition isotherms for the formation of a metal hydride. Three distinct areas are

*J. L. Anderson, M. Bowman LASL, V. A. Maroni, ANL.

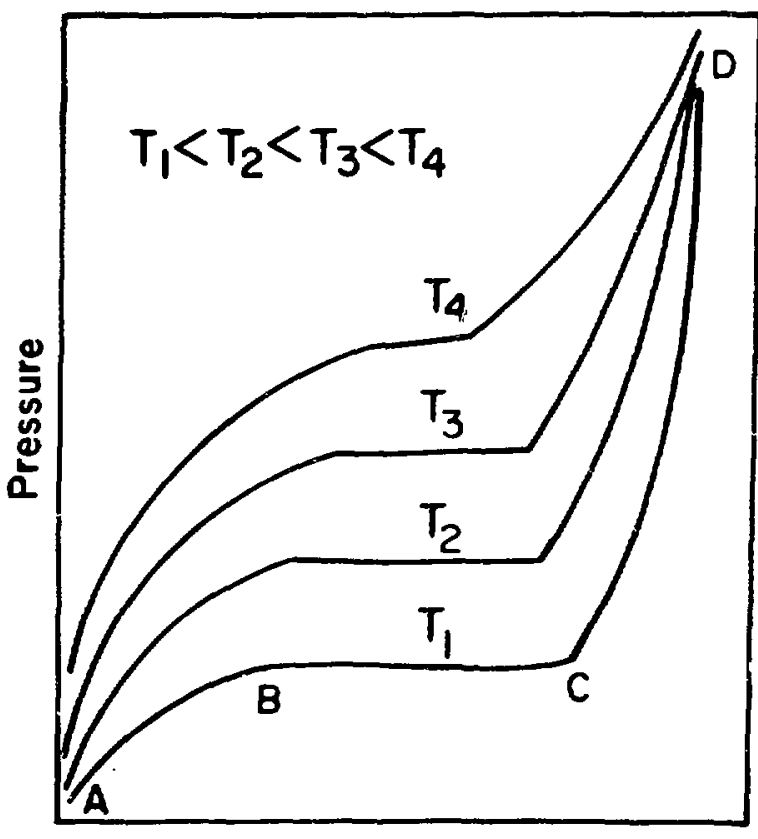

$H / M$ in Solid

Fig. 4.9-1.

Pressure-composition diagram for a typical metal and metal-bydride system. apparent on each isotherin. The steeply rising portion, $A B$, corresponds to a solution of hydrogen in the metal. The reaction for solution of hydrigen in the metal can be written,

$$
\frac{1}{2} \mathrm{H}_{2} \text { (gas) } \rightleftarrows \underline{H}(\text { in solution) }
$$

and the equilibrium constant for the reaction is given by,

$$
K=\frac{a_{H}(\text { In meta1) }}{a^{1 / 2}(\text { gas })}=N_{H} P_{H}^{-1 / 2}
$$

where $a_{H}$ and $a_{H_{2}}$ are the activities of the dissolved hydrogen and the hydrogen gas, respectively, and $\mathrm{N}_{H}$ is the atom fraction of hydrogen in solution Sieverts' law $\mathrm{N}_{\mathrm{H}}=\mathrm{KP}$, states that the solubility of hydrogen in a metal is proportional to the square root of the hydrogen pressure in the dilute solution range.

When saturation is reached (point B), the hydride phase corms. At this point the system becomes invariant, and the two-phase "plateau" region appears for compositions between $B$ and $C$. At point $C$ the metal phase has completely reacted and the hydrogen-deficient hydride phase requires a rapid increase in hydrogen pressure to approach stoichiometry. At higher temperatures, the solubility of hydrogen in the metal increases, the plateau region shortens, and higher hydrogen pressures are required to approach stoichiometry.

It is the Sieverts' law region (AB) that is of interest for the blanket system. To maintain an acceptable tritium inventory and to keep tritium losses to an absolute minimum, very low tritium pressures $\left(\sim 10^{-10}\right.$ Torr $)$ must be maintained over the blanket. Pressure-composition data in the Sieverts' law region for all applicable metal hydride systems are generally measured at relatively high composirions and pressures. These data must be extrapolated over several orders of magnitude to obtain values applicable to the design of a reactor. Because of these extrapolations, Sieverts' law may not be applicable in the pressurecomposition region of interest to the RTPR. At these very low compositions the square root of pressure relationship may be significantly perturbated by "holes" in the liquid metal or by low-level impurities in the blanket system. Until better data are available, however, the design of the tritium recovery system must rely on chemical data which have been subjected to considerable extrapoiation. 
4.9.2.2 Tritium Management and Recovery Schemes. Recent studies ${ }^{\text {t-5 }}$ have addressed the problems associated with tritium management in fusion power plants. A significant conclusion drawn from these studies is that tritium losses resulting from its permeation through the heat transfer circuits could present a serious obstacle to the demonstration of the environmental acceptability of thermonuclear power. This is particularly true for D-T fueled reactor concepts with liquid lithium blankets and niobium-alloy structures, because ef the relatively high hydrogen permeability of niobium ${ }^{3,5}$ and the appreciable solubility of hydrogen in lithium. ${ }^{6}$

Volume 11 of this report indicates that a tritium partial pressure not exceeding $10^{-10}$ Torr in a lithium blanket system represents a tritium level attainable by practical processing methods. This partial pressure is sufficiently low to permit safe operation of the plant.

At least two approaches to this problem exist. One possible solution is the development of multilayered materials for heat exchanger walls. These laminated materials would be fabricated so that at least one of the layers is relatively impermeable to hydrogen. The other approach is to devise a process which for all practical purposes is infinitely efficient in removing tritium from lithium. The latter approach is favored because it reduces the driving force for permeation by minimizing the inventory of tritium in the lithium. In a!! probability a combination of both approaches will have to be employed.

Schemes proposed for separating tritium frorn the lithium blanket include: (1) tritium permeation through a metal membrane, (2) cold trapping, (3) trapping on a solid sorbent, (4) distillation, (5) gas sparging, and (6) liquid-liquid extraction. Watson ${ }^{4}$ has discussed the first five approaches in some detail. It is, however, instructive to summarize briefly the advantages and disadvantages of each method.

The separation of tritium from the lithium by permeation of tritium through a metal "window" has received much attention in recent years. Tritium permeation through a niobium window is considered the most likely possibility. To be reasonably effective, the lithiumtritium mixture should enter the window area at $\sim 1300 \mathrm{~K}$; at this temperature the tritium permeation of niobium is fairly high. ${ }^{7}$ This technique, however, requires the maintenance of a very low partial pressure of tritium on the opposite face of the window. The best way to do this might be to oxidize the $T_{2}$ to $T_{2} O$ which can be collected on a molecular sieve trap. Oxygen, however, will corrode niobium, so a palladium coating would be needed on the back side of the niobium window. This in turn creates uncertainty as to how $T_{2}$ permeation will be affected by any compound formation (i.e., $\mathrm{NbPd}_{3}$ ) at the niobium-palladium interface. The cost of fabricating the lange number (total surface area of several thousand square feet) of niobium-palladium tubes and the expense of certifying such tubes could be prohibitive.

Cold trapping of solid LiT from the lithium blanket at a temperature only slightly above the lithium freezing point $(454 \mathrm{~K}$ ) is not very attractive. The cold traps will require very large surface areas and very long residence times. This increases considerably the inventory requirements of lithium in the blanket. Additionally, good data indicating that cold trapping will reduce the tritium to acceptable levels do not exist. It is not felt that this area warrants further work at this time.

If the cold trap is filled with a metal which forms a more stable hydride than lithium hydride, this metal will tend to extract the tritium from the blanket lithium. Good candidate metals for this purpose are ytrrium, lenthanum, and cerium. The solubility of tritium in yttrium, lanthanum, cerium, and lithium at temperatures in the range $600-1000 \mathrm{~K}$ and low pressures $\left(<10^{-6} \mathrm{Torr}\right)$ has not been determined. Data at higher tritium levels and higher temperatures have been extrapolated to the area of interest. These extrapolated data look encouraging enough to merit further investigation of this technique.

Both distillation and gas sparging have been mentioned as possible techniques for removing tritium from lithium. Based on the available vapor-liquid equilibrium data, Watson ${ }^{4}$ gives a convincing angument for disregarding these techniques. At the tritium levels demanded by the safety requirements, the vapor phase in a distillation or gas-sparging system will actually become enriched in lithium, thereby achieving just the opposite of the desired goal.

In summary, it does not appear that methods which involve evaporation ${ }^{8}$ (distillation); gas sparging, ${ }^{4}$ cold trapping, ${ }^{4}$ or mechanically pumped, permeable menbranes $^{4}$ will sufficiently reduce the tritium concentration in lithium to an acceptable level. Schemes that employ a solid-hydrogen-gettering substance in intimate contact with the lithium appear promising ${ }^{4}$ from the standpoint of reducing the tritium concentrations in the lithium, but practical methods for regenerating the getter need to be developed.

\subsubsection{Molten Salt Contactor}

A process developed for fission reactor fuel processing, which may have application in fusion devices, involves removal of fuel materials from liquid metals by liquid-liquis extraction with a molten salt.9,10 With regard 
to lithium-blanketed fusion reactors, it appears possible to extract lithium hydride ( $\mathrm{LiH}$ ) from lithium by use of a lithium chloride-containing molten salt. In Fig. 4.9-2 has been plotted pressure-composition data for the LiH-LiCl and LiH-Li systems. The LiH-LiCl data (which have been extrapolated over four orders of magnitude in composition) were determined as part of a lithium-hydrogen galkanic cell program." The data for LiH in lithium were recently obtained in a study of the thermodynamics of the LiH-Li system. ${ }^{6}$ The curves in Fig. 4.9-2 imply a molar distribution coefficient for $\mathrm{LiH}$ between $\mathrm{LiCl}$ and lithium of about 6 at $870 \mathrm{~K}$. If the extrapolation is reasonably accurate and holds for the distribution of LiT between $\mathrm{LiCl}$ and lithium over a reasonable temperature range $(700-1000 \mathrm{~K})$, then it should, in principle, be possible to obtain a high tritium recovery rate and a very low steady-state inventory of tritium in the blanket by an extraction process. Figure 4.9-3 shows schematically how this process might be applied to a fusion reactor, whereas Fig. 4.9-4 illustrates in detail the proposed contactor. The low-temperature lithium $(\sim 680 \mathrm{~K})$ and the lithium halide salt (e.g., $\mathrm{LiCl}-\mathrm{KCl}$ eutectic, melting point is $623 \mathrm{~K}$ ) are fed simultaneously into a centrifugal contactor where they are intimately mixed to effect an equilibrium separation of the LiT; separation then occurs by centrifugal action. With adequate staging of the centrifugal sector, complete separation of the lithium and the salt should be possible, because at the temperatures of interest $(680-820 \mathrm{~K})$ the specific gravity ratio of the salt to the liquid metal is greater than a factor of three. A conservative estimate of the degree of processing needed to maintain the tritium partial pressure in the lithium at or below

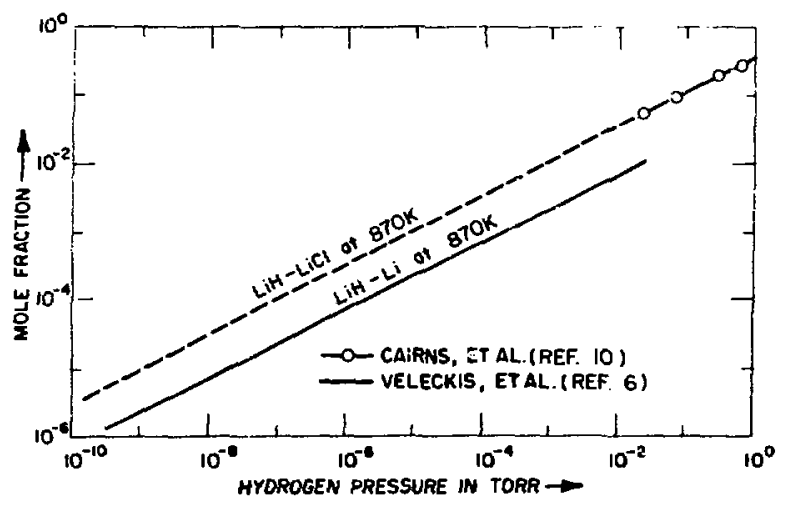

Fig. 4.9-2.

Pressure-composition diagram for LiH-LiCl and LiH-Li system at $870 \mathrm{~K}$ for the Sievert's law region.

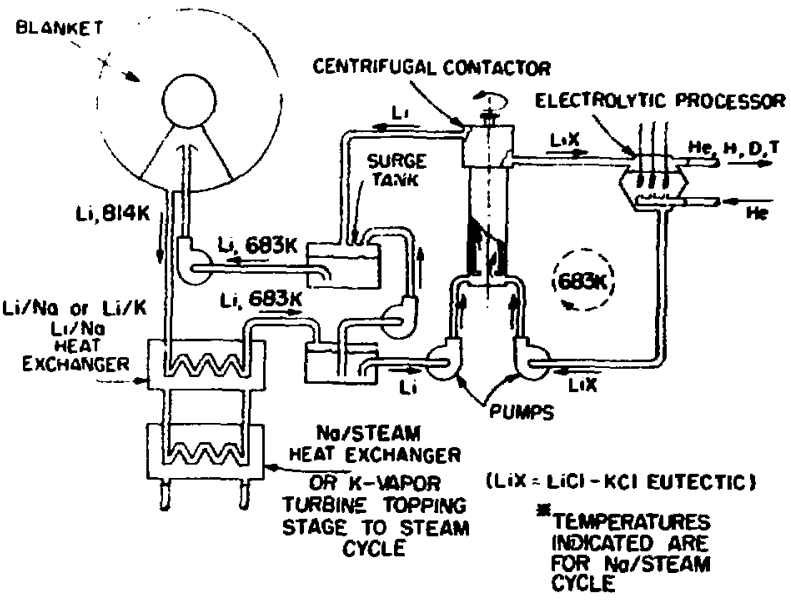

Fig. 4.9-3.

Schematic diagram of a separation process based on the LiH-LiCl-Li system.

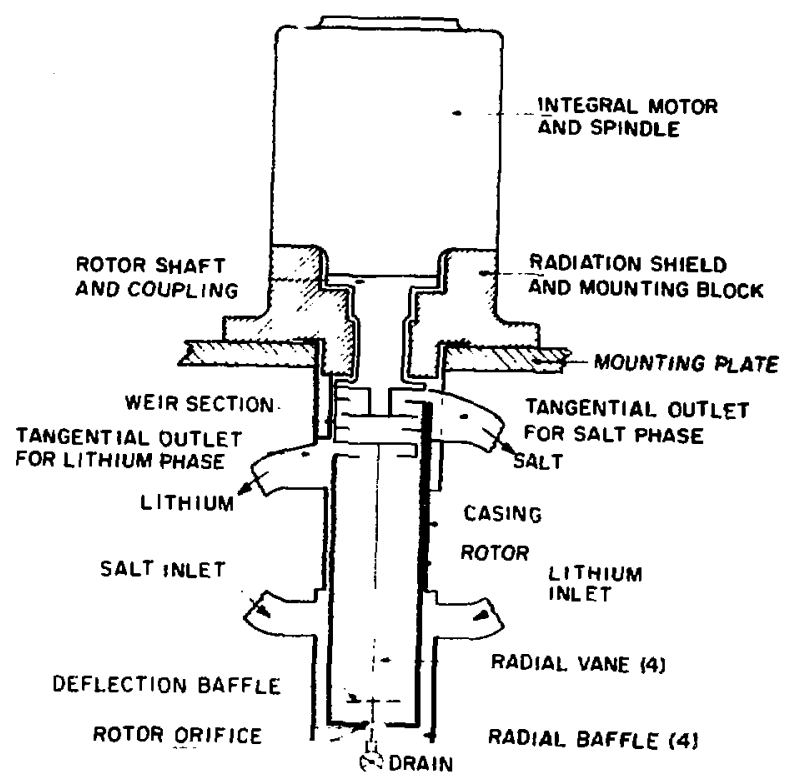

Fig. 4.9-4.

Detailed view of Li/LiCl centrifuge contactor. 
$10^{-10}$ Torr has indicated that the entire lithium flow should be contacted with an equal volume of a salt. This would require 50 conventional contactor units ${ }^{9,10}(50 \mathrm{~cm}$ diameter and $43 \mathrm{~cm}$ high) running at rotor speeds of 1700 $\mathrm{sm}$. Continuous operation of this processing system would require upwards of $1.0 \mathrm{MWe}$.

The salt phase (containing most of the LiT) is circulated from the contactor to a processor and the tritium is either removed electrochemically (by operating the lithium-hydrogen galvanic cell in reverse) or sparged from the salt using DCl(DCl $+\mathrm{LiT} \rightarrow \mathrm{LiCl}+\mathrm{DT})$.

Other liquid-liquid extraction systems exhibit potential for effective separation of tritium from lithium. Furthermore, it may be desirable to avoid potentially corrosive metal halides in the system. For instance, if a low meiting eutectic system can be found which contains a sufficient amount of lanthanum, cerium, or yttrium, these materials, which form a more stable hydride than lithium, could be used to extract tritium from the lithium. Again, no data are available on the solubility of tritium in these metals at the low tritium composition ranges of interest. If the relative stabilities of these systems in the two-phase plateau region of the pressure-composition diagram are compared, these materials appear very interesting. Table 4.9-1 gives the measured equilibrium $\mathrm{H}_{2}$ pressure over these hydrides at temperatures from $600 \mathrm{~K}$ to $1300 \mathrm{~K}$. Also listed are the relative stabilities of each metal hydride system compared to the lithium hydride system at the same temperature. If the reasonable assumption is made that the relative stabilities in the Sieverts' law region show the same trends as are shown in the two-phase region, it is obvious that these metals offer the possibility of an even greater extraction coefficient than the value of 6 reported for the $\mathrm{LiCl}$ system.

A brief survey of the literature on low-melting eutectics of these metals shows that yttrium will form a eutectic containing 31.1 wt\% cobalt, melting at $988 \mathrm{~K}$. Yttrium also forms a eutectic mixture with 33 wr\% copper, melting at $\sim 988 \mathrm{~K}$. A lanthanum eutectic with 16 wr\% nickel melts at $\sim 758 \mathrm{~K}$ and a certain eutectic with 14 wt\% iron melting at $\sim 863 \mathrm{~K}$ has also been reported. Each system is worthy of consideration, depending on the temperature of the lithium at the centrifugal contactors. These systems are also high density and, hence, easily separable from the lithium in the contactors. The tritium could be removed from these materials by a thermal decomposition of the resulting hydride. The total volume of extraction liquid to be handled would probably be less for a metal-metal eutectic system than for the molten salt extraction system.

The liquid-liquid extraction scheme appears to be by far the best technique yet proposed for recovering tritium from the blanket system. A thorough investigation of this scheme incorporating materials problems. mutual

TABLE 4.9-1

EQUILIBRIUM $\mathrm{H}_{2}$ PRESSURE (TORR)

\begin{tabular}{|c|c|c|c|c|}
\hline Temp., $\mathrm{K}$ & Li-LiH & $\mathbf{Y}-\mathbf{Y H}_{2}$ & Le- $\mathrm{LaH}_{2}$ & $\mathrm{Ce}_{-}-\mathrm{CeH}_{3}$ \\
\hline 573 & $3.7 \times 10^{-5}$ & $6.4 \times 10^{-11}$ & $6.4 \times 10^{-9}$ & $7 \times 10^{-5}$ \\
\hline 673 & $5.1 \times 10^{-3}$ & $7.7 \times 10^{-8}$ & $4.2 \times 10^{-6}$ & $4.4 \times 10^{-6}$ \\
\hline 773 & 0.2 & $1.5 \times 10^{-5}$ & $5.1 \times 10^{-4}$ & $5.1 \times 10^{-4}$ \\
\hline 973 & 30 & $2.1 \times 10^{-2}$ & $4.0 \times 10^{-1}$ & $3.7 \times 10^{-1}$ \\
\hline 1173 & $8.2 \times 10^{2}$ & 2.5 & 31.7 & 28.6 \\
\hline 1273 & $2.9 \times 10^{3}$ & 14.9 & 169 & 150 \\
\hline \multicolumn{5}{|c|}{ P(Li-LiH) } \\
\hline & & $\mathbf{P}\left(\mathbf{M}-\mathbf{M} H_{x}\right)$ & . & \\
\hline Temp., K & $\mathrm{Y}-\mathrm{YH}_{2}$ & La-LaH ${ }_{2}$ & $\mathrm{Ce}-\mathrm{CeH}_{3}$ & \\
\hline 573 & $1.7 \times 10^{6}$ & $5.8 \times 10^{3}$ & $5.3 \times 10^{3}$ & \\
\hline 673 & $6.6 \times 10^{4}$ & $1.2 \times 10^{3}$ & $1.1 \times 10^{3}$ & \\
\hline 773 & $1.3 \times 10^{4}$ & $3.9 \times 10^{2}$ & $3.9 \times 10^{2}$ & \\
\hline 973 & $1.4 \times 10^{3}$ & 75 & 81 & \\
\hline 1173 & $3.3 \times 10^{2}$ & 25 & 29 & \\
\hline 1273 & $1.9 \times 10^{2}$ & 17 & 20 & \\
\hline
\end{tabular}


solubilities, consequences to the overall neutronics of the blanket, the actual distribution coefficients, and selection of the optimum extraction liquid (constituents and composition) remains to be performed.

\subsubsection{Removal of Impurities}

In addition to the removal of tritium from the lithium it will be necessary to remove those impurity elements which can be expected to find their way into the lithium on a continuous basis (i.e., oxygen, ca:bon, and nitrogen). Carbon and nitrogen appear to deserve the greatest attention because of their effects on materials compatibility. ${ }^{12,13}$ In the absence of substantive data to the contrary, the assumption has been made that these impurities will be present in the lithium as nitride $\left(\mathrm{Li}_{3} \mathrm{~N}\right)$ and carbide $\left(\mathrm{Li}_{2} \mathrm{C}_{2}\right)$, respectively. In all likelihood the molten salt contactor method will also remove these "saltlike" impurities with a favorable distribution ratio; the molten salt can potentially act as a scrubber for a wide range of impurity species in lithium. The recovery of these impurities from the salt should be relatively straightforward using modern electrochemical technology. More detailed considerations pertaining to the solubility, interactions, and monitoring of species in lithium have been reported elsewhere. ${ }^{12}$

\section{REFERENCES}

1. D. Steiner and A. P. Frazs, "Preliminary Observations on the Radiologic Implications of Fusion Power," Nuclear Safety, 13 373 (1972).

2. E. F. Jünson, "Fusion Reactor Fuel Processing," Princeton Plisma Physics Laboratory Report MATT-901 (1972).

3. R. G. Hickman, "Some Problems Associated with Tritium in Fusion Reactors," University of California Radiation Laboratory Report UCRL-74057 Rev. 1 (1972).

4. J. S. Watson, "A Summary of Tritium Handling Problems for Fusion Reactors," Oak Ridge National Laboratory Report ORNL-TM-4022 (1972).

5. V. A. Maroni, "Selected Problems of Tritium Management Related to CTR," presented at the Short Course in Fusion Power, Princeton Plasma Physics Laboratory. Princeton University, Princeton, New Jersey, Oct. 30- Nov. 3, 1972.

6. E. Veleckis et al., "Physical Inorganic Chemistry Semiannual Report, July-December 1972," Argonne National Laboratory Report ANL-7978 (1973).

7. R. W. Webb, Permeation of Hydrogen Tbrougb Metals, USAEC Report NAA-SR-10462 (July 1965).

8. R. Farookhi and J. E. Rogers, "iNethods of Tritium Recovery from Molten Lithium," Oak Ridge National Laboratory Report ORNL-MIT-66 (1968).

9. J. B. Knighton et al., "Development of a Mixer-Settler for Liquid Metal-Salt Systems," Argonne National Laboratory Report ANL-7810 (1971).
10. G. J. Bernstein et al., "Development and Performance of a High-Speed Annular Centrifugal Contactor," Argonne National Laboratory Report ANL-7969 (1973).

11. E. J. Cairns et al., "Galvanic Cells with Fused-Salt Electrolytes," Argonne National Laboratory Report ANL-7316 (1967).

12. V. A. Maroni, E. J. Cairns, and F. A. Cafasso, "A Review of the Chemical, Physical, and Thermal Properties of Lithium that are Related to its Use in Fusion Reactors," Argonne National Laboratory Report ANL-8001 (1973).

13. C. E. Sessions and J. H. De Van, "Thermal Convection Loop Tests of $\mathrm{Nb}-1 \%$ Zf Alloy in Lithium at 1200 and $1300^{\circ} \mathrm{C}$," Nud. Appl., 9, 250 (1970).

\subsection{ENERGY CONVERSION SYSTEMS*}

\subsubsection{Systems Requirements}

The energy conversion system transports heat generated in the blanket through a series of heat exchangers to the power plant cycle working medium. Suitable coolant conditioning equipment is required upstream and downstream of the power conversion equipment. This includes comparible heat exchangers, flow conditioners, cooling equipment, and pumps. The power conversion equipment must convert the thermal energy delivered to it by the transport system to electrical energy.

\subsubsection{Blanket Cooling Systems}

4.10.2.1 General Description. The blanket coolant, lithium, is circulated through the blanket as shown in Fig. 3.2-1 and 3.2-2. Because of the cyclic nature of the plasma fusion, neither the flow rate (Fig. 4.5-18) nor the outlet temperature (Fig. 4.5-12B) from the blanket is constant. To aid in smoothing both flow and temperature variations, surge tanks are placed upstream and downstream of the blanket. During the time that the flow through the blanket is restricted by the magnetic field, the lithium flow from the pump is shunted to the upstream surge tank and the downstream surge tank continues to supply lithium to the intermediate heat exchanger (Fig. 4.5-15). When the magnetic field is zero, the lithium flows throngh the blanket without severe restriction, and the upstream surge tank will tend to empty while the downstream surge tank will refill.

Two alternative energy conversion systems are proposed: (1) A conventional, low-temperature system, which employs sodiam as an intermediate heat transfer medium, produces steam in a sodium-steam generator;

"T. Bump, T. A. Coultas, P. Dauzvardis, ANL. 
this system is pattemed after the well-developed LMFBR technology', and (2) an advanced power-conversion scheme based on a potassium topping cycle used with a conventional steam cycle. The latter high-temperature scheme has been proposed by $\mathrm{Fraas}^{2}$ and represents a longer range option. For either the conventional or the advanced conversion schemes, the characteristics of the lithium system remain in essence unperturbed; only the lithium and average blanket temperature is higher for the system based on the potassium-vapor topping cycle.

4.10.2.2 Intermediate Hsat Exchanger and Intermediate Heat Transfer Loop. The lithium flows from the blanket and through the downstream surge tank to a heat exchanger. For the low-temperature option, the heat exchange is with sodium. The sodium system is patterned specifically after that proposed by Westinghouse for the 1000 MWe LMFBR. ${ }^{1}$ Minor modifications in that design were necessary to accommodate the power levels and the need for different materials in the primary heat exchanger. Despite these changes, advantage is taken of the engineering work done on the sodium heat exchangers in connection with the LMFBR program. The steam generator is essentially identical in both plants, but the primary heat exchanger for the RTPR plant is somewhat smaller because of the use of niobium-zirconium alloy for heat exchanger tubes as well as the use of lithium rather than sodium primary coolant. The manufacture of such a heat exchanger will require some development. Detailed characteristics of these heat exchangers and other parts of the system are given in Appendix B.

For the high-temperature power plant alternative the lithium primary coolant exchanges heat with potassium in the potassium boiler. This heat exchanger is patterned after that proposed by Fraas. ${ }^{2}$ This system is also described in Appendix B.

The lithium which flows from the heat exchanger is purified and the tritium is extracted by the centrifugal contactors (see Section 4.9.3). The processed lithium is then pumped back to the blanket and the upstream surge tank. Further details of the flow system for both powerconversion options are tabulated in Appendix B.

\subsubsection{Power Pianะ}

4.10.3.1 Advanced Potassium Topping Cycle Power Plant. The conventional power plant represents state-ofthe-art system and can be built without special development. Since the theta-pinch plant is projected to be built several decades hence, it is expected that many advancements will be made towards increasing the high-tempera- ture strength of refractory metals, improving fabrication techniques, as well as developing other technologies required for highly efficient power conversion. An advanced power plant is described by Fraas. ${ }^{2}$ His study was based on a 1.0 GWt fusion plant and supplied potassium vapor at $1250 \mathrm{~K}$ to a potassium turbine. Fraas' design has been scaled-up in size by approximately a factor of 1.8 , and some of the results are given here. The line diagram of this plant is shown in Fig. 4.10-1.

The lithium is pumped from the RTPR blanket to two potassium boilers. The potassium vapor drives two turbines and enters a condenser; where it gives up its latent heat to a steam boiler and twc steam reheaters. The urbines are three-staged, and the overall plant efficiency is rated at $56.4 \%$, with 2 generators producing a gross power of $2030 \mathrm{MWe}$.

The $1273 \mathrm{~K}$ potassium vapor leave the two lithiumpotassium boilers through $3.5 \mathrm{~m}$ ( 90 in) diam headers and branch into four $1.7 \mathrm{~m}$ (66 in) diam pipes that feed each potassium turbine. Two potassium turbine extractions are used; one supplies the regenerative power to the potassium feed heater, and the other drives a turbine-driven feed pump that provides the circulation from the potassium condenser through the feed heater and back to the lithium-potassium boilers.

High pressure water at $2.83 \times 10^{7} \mathrm{~Pa}(4100 \mathrm{psia})$ is converted to steam, which drives the high-pressure steam turbine, and is returned to the first reheater. The reheated steam goes to an intermediate press'sre turbine running on a common shafr with the high-pressure turbine. A second reheat is provided for the lov/ temperature curbine before the steam is condensed. Each stieam turbine has extraction points that reheat the condensate in the feedwater heaters. Two boiler feed pumps complete the circuit.

\section{10.3.2 Conventional Sodium Steam Power Plant. A} conventional steam power plant was chosen for the environmental study. The major environmental impact of a fusion plant results from tritium bred in the blanket and used to fuel the fusion plant. The major leacage of tritium to the atmosphere will occur in the power plant and is strongly dependent upon the design of the heat exchangers used between the blanket and the power generating equipment.

The 3 second duty cycle was also chosen as the model upon which the environmental study is based (Vol. II). The larger plant output ( $4100 \mathrm{MWe})$ and shorter duty cycle $(3 \mathrm{sec})$ is believed to encompass more severe but realistic environmental problems, and therefore represents a more interesting case for environmental analysis. To first order the tritium escape from the plant is a linear 


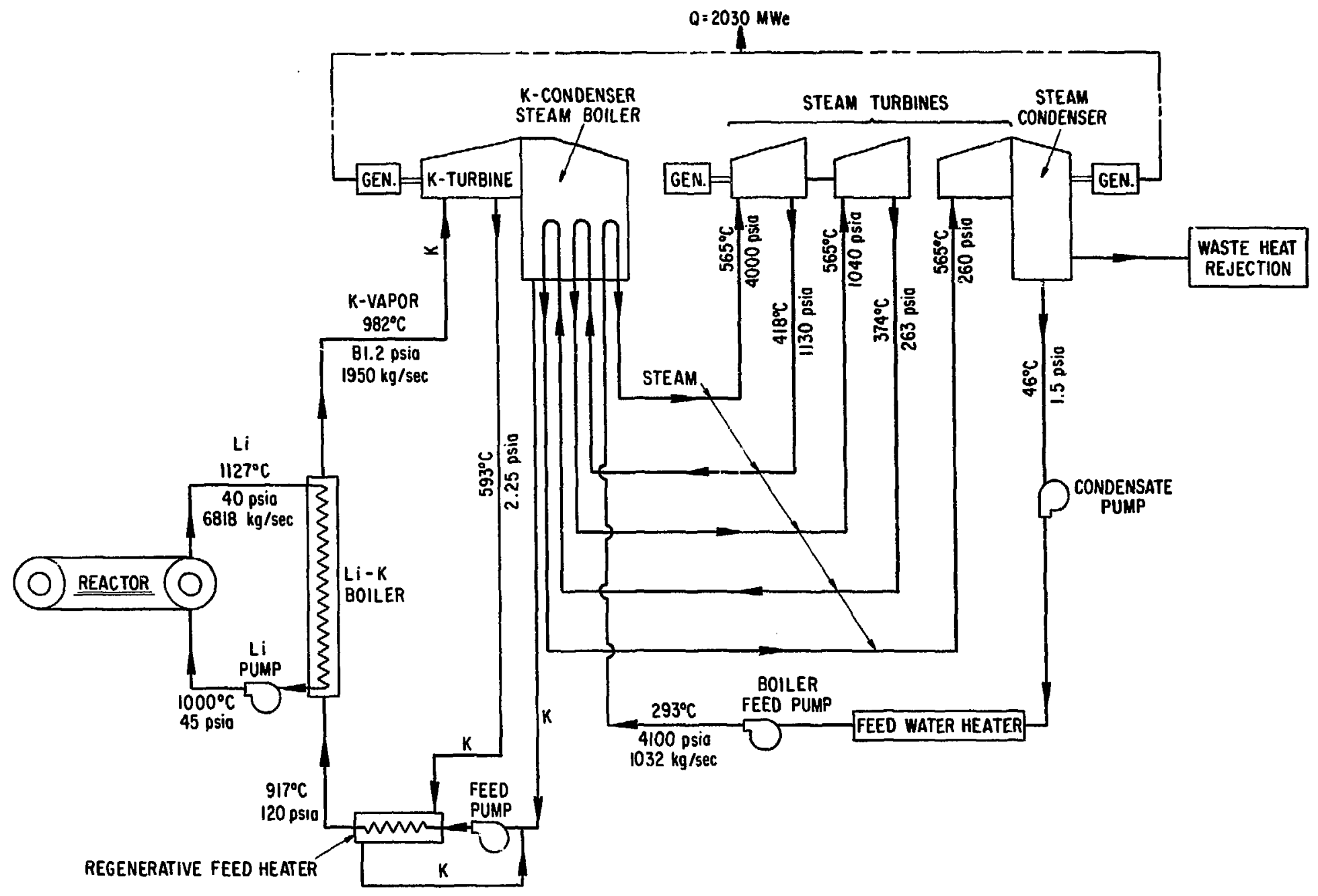

Fig. 4.10-1.

Line diagram of energy conversion system based on potassium-vapor topping cycle. $\left(\tau_{\mathrm{c}}=10 \mathrm{sec}\right)$ 
function of the power output; therefore, the larger plant might pose 2 less trivial environmental problem. For that reason, the plant option having the largest power output and hence the larger heat exchanger areas was selected for the environmental study. If flow, area, and power values are desired for the 10 second cycle, these can in most cases be obtained by simple scaling. On the basis of the foregoing rationale the numerical values present in the remainder of this section pertain to the larger plant $\left(P_{E}=4100 \mathrm{MWe}\right)$ using a minimum duty cycle $\left(\tau_{c}=3.0\right.$ sec).

The steam plant proposed by Westinghouse' was used for the conservative and noncontroversial designs of these heat exchangers. The plant operates at $755 \mathrm{~K}\left(900^{\circ} \mathrm{F}\right)$ maximum steam temperature and does not use reheat. Turbines of this nature are running at this time in many fossil-fueled power plants in the United States. This choice was also advantageous because it utilizes a secondary heat transfer fluid ahead of the steam generator. The major advangage, however, is that the heat exchanger designs are based upon extensive analysis, and that analysis can be carried over directly to the RTPR design. A detailed description of LMFBR power plant is given in Ref. 1, and only a summary description is given here.

The description in Ref. 1 pertains to a 1000 MWe plant, and therefore the RTPR uses four such plants. The heat source for the sodium-cooled fast breeder reactor was designed to produce an outlet sodium temperature of $810 \mathrm{~K}\left(1000^{\circ} \mathrm{F}\right)$; the RTPR plant will produce lithium at an exit temperature at about $820 \mathrm{~K}\left(1016^{\circ} \mathrm{F}\right)$.

A line diagram, illustrating the arrangement of the main systems and components, is shown in Fig. 4.10-2. Pertinent plant parameters are tabulated in Appendix B. right primary lithium loops transfer the heat from the blanket through intermeoiate heat exchangers (IHX) to intermediate sodium loops which, in turn, transfer the heat to the working fluid in eight, once-through steam generators. Since the lHXs contain radioactive lithium on one side, they are located inside the containment boundary. The intermediate sodium, however, passes through the containment boundary to the steam generators lccated in an adjacent compartment, because of its low radioactivity.

Steam from the eight steam generators passes through four turbines from which the exhaust is directed to eight condensers. Condenser pressure is maintained at $0.54 \times 10^{4} \mathrm{~Pa}(0.8 \mathrm{psia})$ by $287 \mathrm{~K}\left(57^{\circ} \mathrm{F}\right)$ cooling water.

The primary reactor coolant system consists of eight main (lithium) loops, including the blanket, surge tanks, shell side of the IHX, primary pumps, and associated piping. At full power, a total of $82 \times 10^{6} \mathrm{~kg} / \mathrm{hr}$ of lithium is circulated through the reactor, to $1 H X s$ and back to the blanket through the pumps. The hot-leg temperature is $820 \mathrm{~K}$ and the cold-leg temperature is $683 \mathrm{~K}$. This system transports $12,000 \mathrm{MWt}$ from the reactor to the intermediate cooling system. For shutdown or emergency conditions an axuiliary cooling system is provided.

The intermediate cooling system also consists of eight loops, and includes the tube side of the IHXs, the shell side of the steam generators, the intermediate sadium pumps, and associated piping. The total sodium flow rate is $202 \times 10^{6} \mathrm{~kg} / \mathrm{hr}$, with a cold-leg temperature of $625 \mathrm{~K}$ and 2 hot-leg temperature of $795 \mathrm{~K}$. This system also transports $12,000 \mathrm{MWt}$ from the IHXs to the steam generators, which are once-through, vertical, shell-and-tubetype units. Feedwater at a flow rate of $20.6 \times 10^{6} \mathrm{~kg} / \mathrm{hr}$ enters the steam generators at $533 \mathrm{~K}$ and exits as $1.66 \times 10^{7} \mathrm{~Pa}$ (2400 psia) and $755 \mathrm{~K}$ superheated steam.

\section{REFERENCES}

1. "LMFBR Demonstrazion Plant; LMFBR Follow-On Study," Westinghouse Report WARD-2000-97 (March 1969).

2. A. Frass, "Conceptual Design of the Blanket and Shield Region and Related Systems for a Full-Scale Toroid Fusion Reactor," Oak Ridge National Laboratory Repurt ORNL TM 3096 (May
1973). 


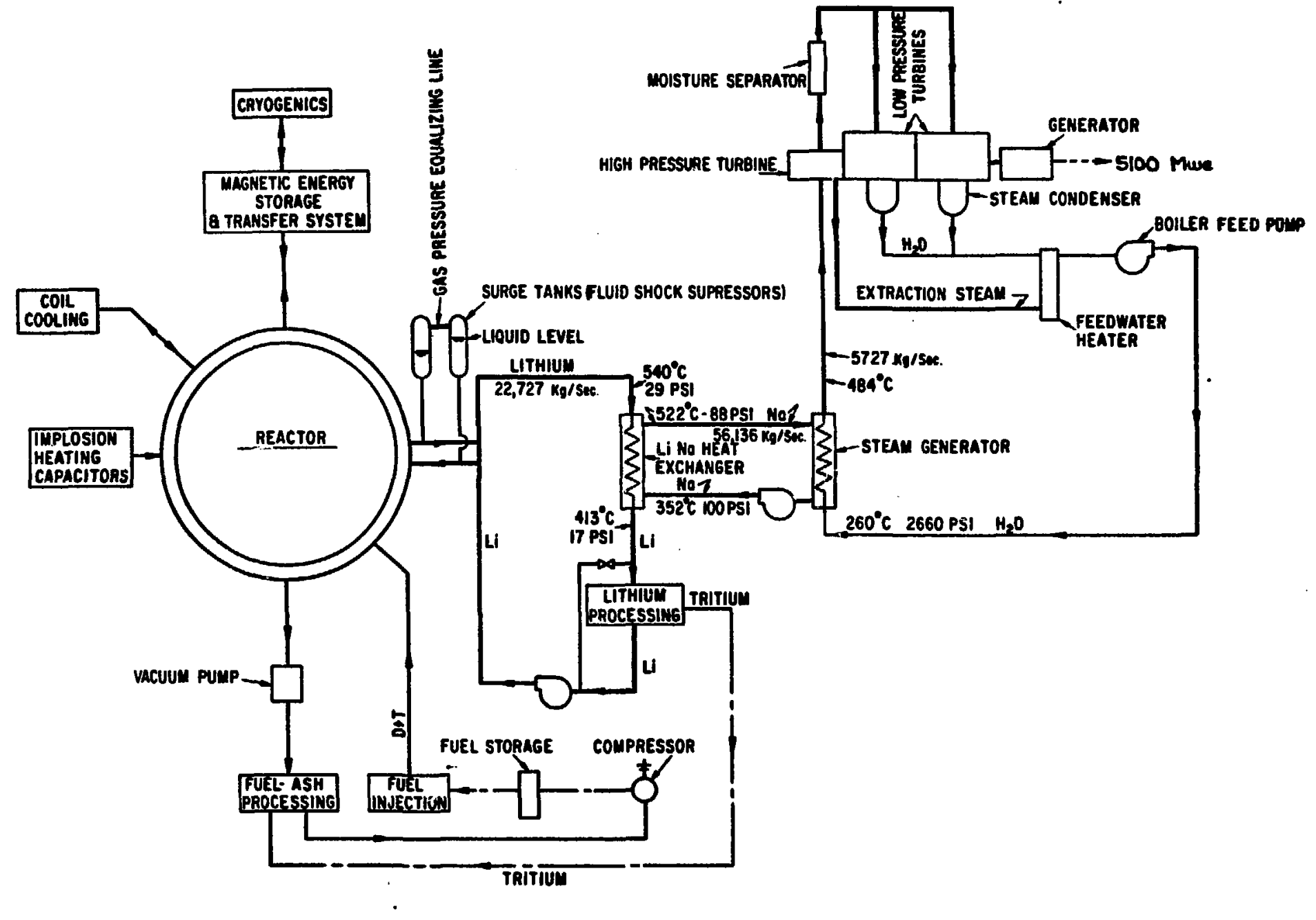

Fig. 4.10-2.

Line diagram of energy conversion system based on the sodium-steam cycle. $\left(\tau_{\mathrm{c}}=3 \mathrm{sec}\right)$ 


\section{MATERIALS*}

\section{S.1 FIRST WALL}

An extensive report ${ }^{t}$ discusses problems of first-wall materials associated with pulsed reactors, as well as the Tokamak and mirror machines, No obvious fundamental deterrents to fusion reactor development were revealed, insofar as material limitations are concerned, but a deficiency of the required physical property data for the first-wall conditions was noted. More research and development will be needed on refractory metal alloys for all CTR concepts and on insulators, in particular, for the pulsed systems.

The first wall of the RTPR will be a composite material containing an insulater lining on a metal backing. The special features of insulators and of metals are discussed below in Secs. 5.1.1 and 5.1.2 with relation to first-wall conditions and material operating requirements. This introduction considers the composite wall material as a whole.

The first wall of the RTPR will be lined by an insulator to support the emf which develops during implosion heating. This insulator will be thin $(0.3 \mathrm{~mm})$ and will be supported by a backing (1.0 mm thick) composed of refractory metal alloy. Energy will be deposited in both the insulator and its metal backing. Molten lithium will cool the first wall and will be in direct contact with the refractory metal surface. Both the insulator and the refractory metal support will sustain neutron radiation damage. A temperature spike during the plasma burning and cooling stages will induce thermal stresses because of constraints internal to the first wall structure, and differential thermal expansion of the insulator and the metal backing. The thermal and mechanical response of the first wall have been estimated quantitatively in Sec. 4.4.

Chemical reaction between the insulator and the refractory metal backing is aetermined by the thermodynamic properties of the chemical constituents of the insulator and metal. Fortunately, a number of favorable and well-documented possibilities exist, such as $\mathrm{Al}_{2} \mathrm{O}_{3}$ on niobium or molybdenum. Although a thin reaction layer between the insulator and the metal is desired to assure goor adhesion, progressive oxidization-reduction at this interface may lead to degradation of the interface and

-F. Clinard, W. Green, LASL; A. T. Sarthanam, ANL. adversely affect the properties of the insulator-metal composite. Chemical compatibility between the blanket materials and molten lithium must also be 2ssured; the behavior of niobium in lithium is discussed in Sec. 5.3.

Thermal conduction within the first wall will be inade quate to carry away the heat without significant temperature increases during the plasma burning and cooling stages (see Sec. 4.4.5). This thermal energy must be dissjpated between plasma-burning pulses. The insulator will otherwise blister, flake, or crack, exposing the plasma to the refractory metal alloy; the shape of the impiosionheating electric field would be destroyed; and the RTPR would tail to function. Differential swelling between the insulator and the metal caused by irradiation-induced voids could cause separation of the ceramic-metal interface. Swelling, however, uccurs only after lange neutron fluences $\left(\sim 10^{22} \mathrm{n} / \mathrm{cm}^{2}\right)$ and is entirely determined by the imposed temperature and fluence, and the microstructure of the material. The predictability of interfacial failure by swelling implies periodic (every few years) replacement of the first wall.

The frequent temperature transients in the first wall which accompany the burning and cooling stages of the plasma will generate cyclic fatigue stresses that can lead to surface roughening by intrusion-extrusion formation. Intrusions-extrusions on the interface might damage the interfacial bonding and therefore degrade the first-wall heat transfer characteristics. Again, the solution is simply to replace the first wall. The first-wall life could be lengthened by controlling the interfacial energies of the insulator and refractory metal by use of graded insulators or by alloying, thereby suppressing intrusion-extrusion formation.

\subsubsection{First-Wall Insulator}

The RTPR design utilizes alumina $\left(\mathrm{Al}_{2} \mathrm{O}_{3}\right)$ as the electrically insulating liner of the irst-wall and was chosen because it is a well-characterized, high-temperature dielectric, which possesses many desirable properties for use in the RTPR. Alumina is not necessarily the best choice, however, and many other materials are under consider: tion. Ingeneral, the insulator should have good thermal and electrical properties at high temperatures, tolerate irradiation without unacceptable degradation of these 
properties, not undergo lange dimensional changes as a result of irradiation and the build-up of transmutation products, and resist chemical attack at elevated temperatures by hydrogen isotopes. The insulator must also be chemically and mechanically compatible with the metal substrate. In the following paragraphs first-wall operating conditions and their relationship to the insulator are discussed; possible solutions to expected insulator problems are also given.

5.1.1.1 Effects of Hign Temperature on Electrical and Structural Properties. Depending on the power conversion system used in the RTPR, the operating temperature of the first-wall insulator is in the range $800-1300 \mathrm{~K}$ with excursions of several hundred degrees accompanying each power pulse. The heat from the plasma cooling phase contributing to this excursion is $137 \mathrm{~J} / \mathrm{cm}^{2} /$ pulse, or 137 $W / \mathrm{cm}^{2}$ for a 1-sec plasma heat dump. The contributions during the burn from the absorption of bremsstrahlung $\left(1432 \mathrm{~J} / \mathrm{cm}^{3} /\right.$ pulse), gamma rays $\left(64 \mathrm{~J} / \mathrm{cm}^{3} /\right.$ pulse), and neutron heating $\left(156 \mathrm{~J} / \mathrm{cm}^{3} /\right.$ pulse) translate into a tempetature rise of $\sim 300 \mathrm{~K}$ (maximum) in the insulator. The temperature rise associated with the plasma cooling stage is controllable (see Figs. 4.4-3).

Electrical resistivity and dielectric strength of insulators normally decrease at elevated temperatures, whether charge transport is by electronic or ionic motion. Reactor design considerations require an electrical resistivity $\geqslant 10^{6} \Omega \mathrm{cm}$ and a dielectric strength $\geqslant 100 \mathrm{kV} / \mathrm{cm}$. The better insulators can meet the resistivity requirement, but their dc dielectric strength is often less than $100 \mathrm{kV} / \mathrm{cm}$ at RTPR operating temperatures. Fortunately, the voltage in the pulsed reactor is applied across the first-wall insulator for less than $1 \mu \mathrm{sec}$, and dielectric strength at elevated temperatures for many materials is considerably higher under pulsed conditions. ${ }^{2}$ An experiment is under way at $\mathrm{LASL}^{3}$ to evaluate electrical breakdown in promising insulators under pulsed conditions. Preliminary results ${ }^{4}$ obtained for polycrystalline alumina given in Fig. 5.1-1 show that its dielectric strength is greatly enhanced in the pulsed mode. Thus it may be possible to use a first-wall insulator thinner than $0.3 \mathrm{~mm}$, unless irradiation effects prove to be deieterious. These results are preliminary, however, and definite conclusions must await the results of experimentation currently in progress.

Some insulators may undergo stoichiometry changes at first-wall temperatures with resultant degradation of electrical properties by introduction of charge carriers and trapping centers. The anticipated thermal gradients could also reduce insulator performance by introducing gradients in stoichiometry or impurity concentration.

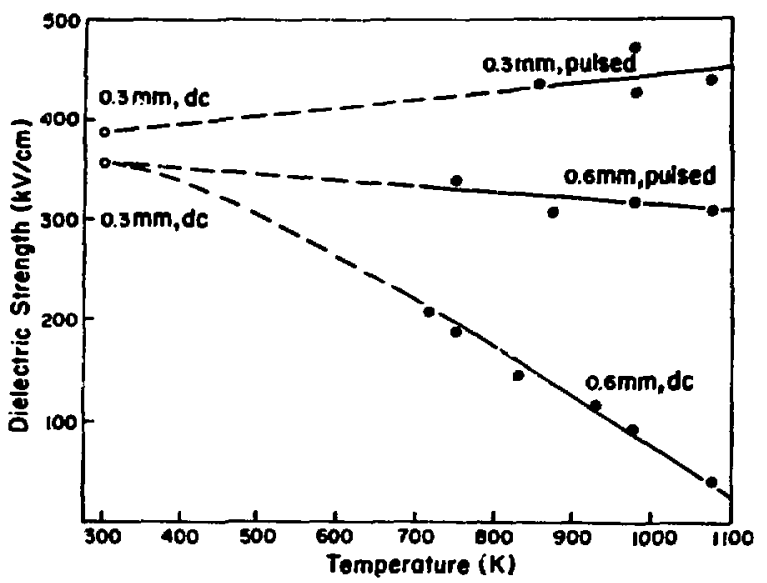

Fig. 5.1-1.

Pulsed dielectric strength of polycrystalline alumina os temperature.

The thermal pulses to which the insulator will be subjected will give rise to stresses which could cause cracking or spallation. These tendencies can be minimized by using a material with high dielectric strength (thinner layers can be used), high hea: capacity, and high thermal conductivity. The thermal cos ductivity needed at operating temperature, $\sim 0.1 \mathrm{~W} / \mathrm{cm} \mathrm{K}$, is attainable with several crystalline ceramics (including $\mathrm{Al}_{2} \mathrm{O}_{3}$ ), but glassy naterials exhibit characteristically poor thermal conductivities. As described in Sec. 4.4, temperature excursions and thermal gradients in the first-wall insulator are large but not prohititive. Studies are in progress to determine the consequences to insulator-metal composites of repeated (thermal) stress pulses, such as those predicted for the RTPR.

Another structural problem may arise if the neutral fuel gas in contact with the wall during the plasma heat dump is at a temperature significantly higher than that of the wall; chemical sputtering or erosion may then occur. The resistance of a insulator to such sputtering is a function of its thermodynamic stability and surface reaction rates and is difficult to predict theroretically. The seriousness of this potential problem cannot be judged until the gas temperature at the first wall is more accurately known.

5.1.1.2 Effects of Neutron Bombardment on Electrical and Structural Properties. The first-wall insulator will in one year be subjected to a fluence of $2.6 \times 10^{22} \mathrm{n} / \mathrm{cm}^{2}$, of which $\sim 20 \%$ have energies close to $14 \mathrm{MeV}$. The neutron 
dose is pulsed, with neutrons striking the wall in $\sim 0.1 \mathrm{sec}$ bursts every 10 sec. Neutron irtadiations at this level will result in changes in electrical and structural properties.

Electrical resistivity and dielectric strength may decrease beccuse of the generation of point defects (which can serve as electron and hole traps, or enhance ionic conductivity) and free electrons (which can populate the traps and may - contribute to electronic charge transport). Since volrage is applied almost $10 \mathrm{sec}$ after irradiation, much of the damage may thermally anneal by the time the insulating properties are needed. Insulators which anneal rapidly could prove relatively insensitive to pulsed reactor radiation damage insofar as electrical properties are concerned. An experiment is being conducted at LASL $^{3}$ to evaluate the recovery linetics of insulating properties after pulsed ion irradiation. Information obtained in this study may allow the adjustment of material parameters so as to accelerate recovery of dielectric properties. For example, efforts might be directed toward manipulation of the defect structure so as to alter charge trapping and detrapping kinetics.

The build-up of transmutation products in the insulator will be significant. Calculations (see Sec. 4.5.2) show that $\mathrm{Al}_{2} \mathrm{O}_{3}$ after one year under operating conditions will contain $\sim 0.24$ at \% each of $\mathrm{H}, \mathrm{He}, \mathrm{C}$, and $\mathrm{Mg}$. An excess burnup of oxygen (relative to aluminum) of $\sim 200$ atomic ppm is expected to occur. The role of impurities from neutron-induced transmutations on electrical properties will depend on whether impurities remain in solution or precipitate. Impurities may behave similarly to displacement-generated point defects if they remain in solution. If precipitated, transmutation products could cause localized field effects and premature dielsctric breakdown. Transmutation products might also degrade stnictural properties. It will be necessary to evaluate the detailed behavior of transmutation products in insulators of interest, with respect to their tendency to remain in solution, precipitate, or escape by diffusion or migration. Such behavior can be expected to differ vastly from one insulator to another. For example, transmutation-induced helium could escape rapidly from some glasses, but might precipitate as gas bubbles in ceramics. The use of gettering or precipitating agents in the insulator to control deleterious effects of transmutation products may be desirous. The effects of transmutation impurities can be simulated by ion injection or by using sputtering techniques to build up insulator samples while simultaneously intrcducing impurities.

Neutron bombardment will create vacancies and interstitial atoms which, as isolated point defects, can result in volume changes. These changes are usually small and may anneal out at the operating temperatures expected.

In anisotropic materials, defect agglomeration products may preferentially orient so as to cause swelling in preferred directions. For example, it has been found ${ }^{5}$ in fast-fission reactor exposures of single-crystal $\mathrm{Al}_{2} \mathrm{O}_{3}$ at $1023 \mathrm{~K}$ that swelling alon, the c-axis is $\sim 1.8 \%$ after a fluenc: of $\sim 5 \times 10^{21} \mathrm{n} / \mathrm{cm}^{3}(>0.1 \mathrm{MeV})$, whereas along the a-axis the swelling is only $\sim 0.2 \%$. Sach swelling in polycrystalline materials with randomly oriented grains has led to grain boundary separation." The problem can possibly be alleviated by orienting the grains with their maximum swelling directions perpendiculas to the substrate, in which direction some dilation can be tolerated. Such an orientation may be obtained by sputtering deposition techniques. ${ }^{6}$ Insulators of cubic crystalline structure are not subject to anisotropic swelling and may prove preferable to $\mathrm{Al}_{2} \mathrm{O}_{3}$. The use of fine-grain material reduces swelling in $\mathrm{Al}_{2} \mathrm{O}_{3} .{ }^{7}$ For this case, also, sputrering techniques could be helpful because sputtered materials of ten have very small grains. ${ }^{8}$

Another mechanism of swelling by agglomeration of vacancies into voids, often assisted by precipitation of insoluble gas atoms, can produce swelling of several volume percent. Recent evidence indicates that metals can be stabilized against swelling by the introduction of small precipitates that apparently induce a more efficient recombination of vacancies and interstitial atoms. ${ }^{9}$ Because of the similar mechanisms of swelling in metals and ceramics, this technique holds promise for controlling swelling in insulators. A study is planned at LASL to evaluate the suppression of swelling in crystalline insulators by the controlled introduction of precipitates.

A small amount of first-wall swelling can be tolerated by designing the reactor core to accomodate slight dimensional changes. Of course, the insulator and its metal substrate must undergo similar dimensional changes to prevent separation or fracture.

Another insulator problem may be neutron spustering. Although not so serious as charged-particle sputtering (to be avoided in the pulsed reactor by controlled relaxation of the magnetic field in conjunction with a neutral gas layer), neutron sputtering must still be considered as a source of long-term damage. The critical parameter is the number of atoms sputtered by an incident neutron (sputtering yield). Calculations suggest that the sputtering yield will be between $10^{-5}$ and $10^{-7}$. A value of $10^{-5}$ would result in an acceptable erosion rate of $\sim 1.0 \mu \mathrm{m} / \mathrm{y}$. The rather sparse experimental data seem to poirt to a sputtering yield of $\sim 10^{-3}$, equivalent to an erosion raie of 10 $\mu \mathrm{m} / \mathrm{yr}$. Although toletable, this sputtering rate could lead to contamination of the plasma. 
Heat transport in opaque insulators is almost wholly by lattice (phonon) conduction, and lattice damage by neutron irradiation of crystalline materials disrupts this conduction. Hence, another problem may be a decrease in thermal conductivity resulting from radiation damage. For example, it has been found ${ }^{6}$ that $\mathrm{Al}_{2} \mathrm{O}_{3}$ suffers a loss in thermal conductivity by about a factor of two after exposure to a fission reaction fluence of $10^{22} \mathrm{n} / \mathrm{cm}^{2}$ at $973 \mathrm{~K}$. The higher temperature expected for the first-wall insulator may accelerate the annealing of damage.

\subsubsection{Effects of Bremsstrahlung and Gamma Irradia-} tion. The effect of photon bombardment on insulators is highly variat'ie; some dielectrics (mainly alkali halides) have been found to suffer severe displacement damage from ionizing radiation, but the mechanism thought to be responsible (the Pooley-Hirsch effect) is not operative in most ceramic materials. Usually, lattice damage from photons results only from the rather inefficient process of high-energy photoelectron impacts with ions. Photoninduced displacements, therefore, could be caused only by high-energy gamma rays, and this contribution is calculated to be small relative to neutron induced displacements. The creation of photoelectrons, however, will be much more plentiful than the ionization electron charge transport. Plans are under way at the Brookhaven National Laboratory to evaluate electrical effects in insulators during and after irradiations which simulate these cenditions (elecrron and bremsstrahlung bombardments).

\subsubsection{First-Wall Metals}

The main function of the first wall in any fusion reacro: is to provide a barrier between the plasma and the surrounding blanket. Economic operation of the reactor demands that the wall material maintain its integrity while exposed to high fluxes of 14-MeV neutrons, energetic neutral and/or ionized particles, and ultraviolet and visible radiation. In the theta-pinch design, the insulator lining will receive all of these; the metal backing will receive only neutrons and secondary particles. A total neutron flux of $8.16 \times 10^{15} \mathrm{n} / \mathrm{cm}^{2} \mathrm{sec}$ will impinge on the first wall. Momentarily during the puise heating, the wal! temperature may rise $200-300 \mathrm{~K}$ above the nominal value. $A$ number of materials problems may be encountered in service that will limit the useful life of the wall. Those associated with the composite material interaction of metal and insulator were discussed at the beginning of Sec. 5.1. Features intrinsic to the first wall metal are considered in this section. For example, the swelling of the metal that results from vo:d formation and transmutation-produced helium may severely reduce the ductility. The wall may also lose its strength and ductility from corrosion by coolant and from plasma erosion processes (sputtering and blistering). The seriousness of each problem depends on the design details of the reactor and will be considered in separate subsections.

5.1.2.1 Effects of Cyclic Stress and Fatigue on Strength. The mechanical stresses in the first wall have bien estimated quantitatively in Sec. 4.4. These show that the metal in the first wall must sustain significant tensile stresses during a large fraction of time; these stresses are cyclic. Hence, both the creep and fatigue response of the metal will be important.

High teinperature ductility is an important engineering parameter, because it is a measure of the ability of the metal to deform and accommodate stresses without fracture. Voids and dislocations strengthen the metal at lower irradiation temperatures; the ductility, however, is severely reduced. Data on the mechanical properties of refractory metals subjected to neutron irradiation at fluences and energy spectra of interest to fusion reactor designers are practically nonexistent. Helium embrittlement can be a serious problem in metal systems. Vanadium alloys show evidence of helium embrittlement at temperatures above $1000 \mathrm{~K} .{ }^{10}$ Table $5.1-1$ shows that the molybdenum alloy TZM, does not show embrittlement from helium at $1073 \mathrm{~K}$, but this does not mean that embrittlement will not occur at higher or lower temperatures.

A nother property important to design of materials for elevated temperature application is the creep response to a stress. One useful measure of this is the stress required for $1 \%$ creep in $10,000 \mathrm{~h}$ ( 1.14 year). Creep-rupture properties are known for refractory metals only in the unirradiated condition and are shown in Fig. 5.1-2 (data for steels and nickel alloys are included for comparison).

TABLE 5.1-1

\section{EFFECT OF HELUM ON DUCTILITY AT $1073 \mathrm{~K}^{\text {II }}$}

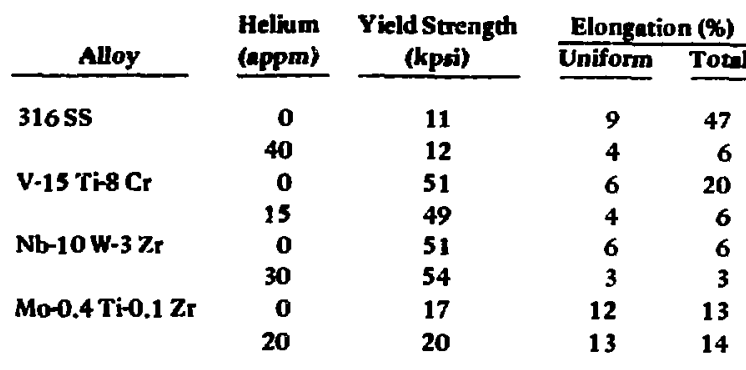




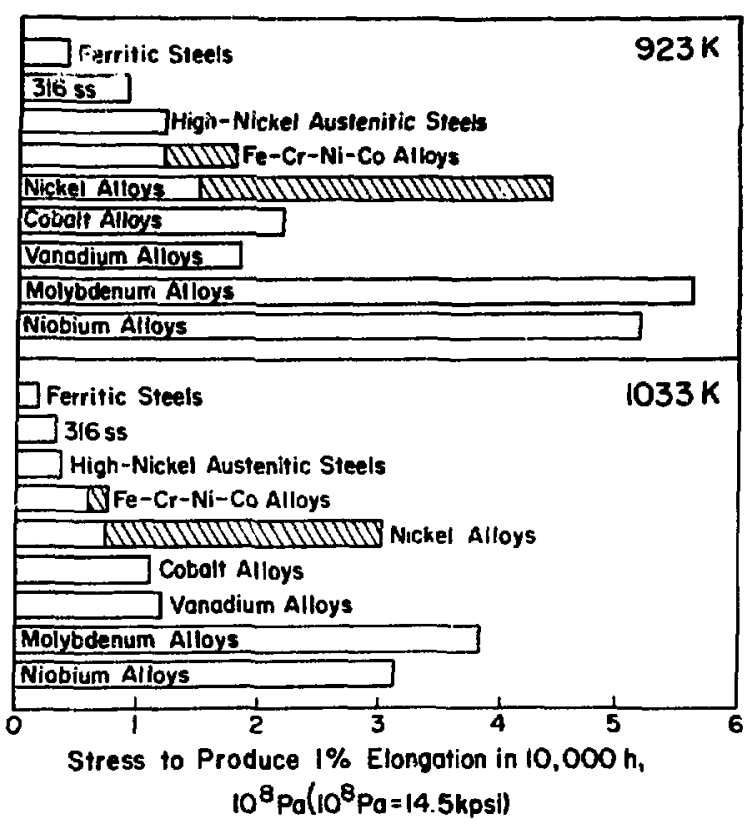

Fig. 5.1-2.

Summary of $1 \%$ creep strength for various alloys at $920 \mathrm{~K}$ and $1030 \mathrm{~K}^{14}$

Molybdenum and niobium alloys are superior to vanadium alloys in this regard. These values for creep rupture strength, however, may have no relation to the in-reactor creep properties, especially if the material bas poor resistance to helium embrittlement.

It is well documented ${ }^{12,13}$ that metals under fastneutron bombardment deform by creep at rates much higher than expected from thermal considerations alone. Although this behavior could restore some of the ductility lost by lattice strengthening, the design must accommodate the change in wall dimension that results from this deformation process.

High-cycle fatigue strength of the wall material is particularly significant to the pulsed theta-pinch design inasmuch as $1 \mathrm{yr}$ of RTPR operation will involve $\sim 10^{6}$ stress cycles. No data however, presently exist for refractory metals at the required temperatures, either in the irradiated or unirradiated condition.

Since the first wall is in direct contact with the blanket coolant, the foremost consideration in the choice of material is chemical compatibility with the coolant. In the present design, lithium has been selected as the cooiant. Refractory metals have been found ${ }^{15}$ to possess maximum corrosion resistance to lithium. Nickel-base alloys are not stitable unless protected by cladding, because of the high solubility of nickel in lithium.

The next step in material selection is consideration of such properties as strength and ductility at elevared temperature, ease of fabrication, low neutron absorption cross section, low induced radioactivity, thermal conductivity, iow thermal expansivity, and other physical properties. Four serious possibilities for the metal choice in the first wall exist; the refractory metal alloy systems (molybdenum and niobium) and vanadium and stainless steels are the only materials with adequate strength, neutronic propertics, and natural abundances which are commensurate with the requirements of the RTPR. (Precipitation-strengthened alloys would likely "over age" to a low strength state.) Figure 5.1.2 compares the strengths of a number of candidate alloys. Vanadium is useful to about $1100 \mathrm{~K}$ which is somewhat low considering that the first wall will be exposed to temperature pulses with each burning and cooling stage of the plasma. Stainless steels are restricted to temperatures below about $900 \mathrm{~K}$. Furthermore, stainless steels have very low thermal conductivities compared to molybdenum, niobium, or vanadium, and the plasma cooling stage may have to be extended. The choice is restricted to molybdenum and niobium. Niobium is preferred for its ease of welding and forming, but other considerations are likely to determine which alloy system, molybdenum or niobium is a better choice. Large size structures of niobium alloys have been fabricated for the space program. These factors make $\mathrm{Nb}-1 \% \mathrm{Zr}$ a popular choice for the first wall. Molybdenum fabrication is difficult at present, but careful design may minimize the problems. Also, the production of "ductile" molybdenum on an experimental scale raises the hope that it could be an alternative choice for the first wall. Experience in the development of materials for space reactors cooled by liquid lithium showed the need to alloy the refractory metals with a strong oxide former, such as zirconium, to avoid intergranular attack. From the point of view of induced radioactivity, vanadium is the best choice. ${ }^{16}$

5.1.2.2 Radiation Effects on First Wall Metal. High temperature radiation damage will cause swelling and high temperature embrittlement. Swelling itself is not damaging to the RTPR as long as dimensional aliowances can be made. Differential swelling in mechanically-constrained situations would cause stresses, but good mechanica] design should reduce these stresses to manageable levels. The bombardment of the wall by energetic neutrons causes displacement damage. In the present design the atom displacement rate is expected to be $\sim \mathbf{4 0} \mathrm{dpa} / \mathrm{yr}$ (displacements per atom per year). Vacancies and 
interstitial atoms are produced in equal numbers, and most of these recombine almost instantaneously. Of the few that remain, the interstitial atoms are preferentially attracted to dislocations, which leave the vacancies to cluster into three-dimensional cavities called voids. Table 5.1-2 shows that the swelling per year in the RTPR would be at most a few percent.

The range of temperatures at which swelling occurs in metals is large (0.25-0.5 homologous temperature) and will lie within the expected operating temperature of the fusion reactors. In niobium, swelling from fission neutrons has been found to occur between $700 \mathrm{~K}$ and $1200 \mathrm{~K}$ with a maximum near $900 \mathrm{~K} .{ }^{17}$ Although it is known that swelling increases with increased neutron exposure, few data are available on the refractory metals. The ion simulation studies of Brimhall and Kulcinski ${ }^{18}$ on niobium at $1100 \mathrm{~K}$ and $1200 \mathrm{~K}$ show that swelling saturates at $4.5 \%$ at $1100 \mathrm{~K}$ and $7-10 \%$ at $1200 \mathrm{~K}$ for doses above $100 \mathrm{dpa}$. This behavior is encouraging in the sense that after about $1 \mathrm{yr}$ of RTPR operation no further increase would occur in the linear dimensions of a niobium wall. However, caution is necessary in applying these results directly, since the above experiments did nor consider the effect of transmutation-produced helium on void formation. Moreover, the swelling behavior of metals can be seriously affected by the impurity content of the metal. ${ }^{19,20}$

Since swelling leads to change in the linear dimensions of the wall, and differential swelling rates between the insulator and the metal backing in the first wall can produce stresses in the wall that could limit its lifetime, an economic advantage exists in producing a first-wall composite that is matched in swelling characteristics. Experiments have consistently shown that swelling can be varied by a slight change in the chemical composition of the alloy or by heavy cold-working of the metal. For example, additions of as little as $1 \%$ titanium have been found to reduce the swelling of commercially-pure vanadium by a factor of $100 .{ }^{21}$ It is quite possible that furure experimental efforts will show suitable alloying elements which will eliminate differential swelling between niobium and molybdenum and the ceramic insulator in the RTPR first wall. The introduction of high number density of dislocations by cold work has been found to reduce swelling in a number of materials. It is, however, important that the dislocation structure remains stable at the reactor operating temperatures, since any rearrangement of dislocation structure can lead to large changes in swelling rate. Recent work suggests that cyclic stressing also influences void formation.

In addition to the displacement damage, the fastneutron flux can generate gaseous and solid products in the wall material through nuclear transmutation reactions. For example, the helium production rate in niobium is $59 \mathrm{ppm} \mathrm{He} / \mathrm{yr}$. Table $5.1-3$ summarizes the amounts of helium, hydrogen, and other solid products expected in 1 yr of operation for a toral neutron flux of $3.7 \times 10^{15} \mathrm{n} / \mathrm{cm}^{2} \mathrm{sec}$. The helium generation rate in a fusion reactor wall is $>0$ to 200 times larger than in fission

TABLE 5.1-2

\section{VOID AND SWELLING DATA FOR NEUTRON-IRRADIATED NIOBIUM ${ }^{17}$}

\begin{tabular}{|c|c|c|c|c|c|}
\hline \multirow[b]{2}{*}{ Material } & \multicolumn{2}{|c|}{ Irradiation } & \multicolumn{3}{|c|}{ Void Parameters } \\
\hline & $\begin{array}{c}\text { Temperature } \\
\text { (K) }\end{array}$ & $\begin{array}{c}\text { Fluence } \\
\text { (neutrons } / \mathrm{cm}^{2} \text { ) }\end{array}$ & $\begin{array}{l}\text { Concentration } \\
\text { (voids } / \mathrm{cm}^{3} \text { ) }\end{array}$ & $\begin{array}{c}\text { Average } \\
\text { Diameter } \\
(\AA) \\
\end{array}$ & $\begin{array}{c}\text { Volume } \\
\text { Fraction } \\
\text { (\%) }\end{array}$ \\
\hline \multirow[t]{3}{*}{ Niobium } & 698 & $2.5 \times 10^{22}$ & $1.6 \times 10^{17}$ & 70 & 3.1 \\
\hline & 858 & $2.5 \times 10^{22}$ & $2.1 \times 10^{17}$ & 71 & 4.8 \\
\hline & 1063 & $2.5 \times 10^{22}$ & $2.8 \times 10^{15}$ & 186 & 1.04 \\
\hline \multirow[t]{3}{*}{ Niobium } & 743 & $3.9 \times 10^{20}$ & $3 \times 10^{16}$ & 20 & 0.01 \\
\hline & 923 & $5.5 \times 10^{20}$ & $8 \times 10^{15}$ & 60 & 0.09 \\
\hline & 1023 & $4.1 \times 10^{20}$ & $5 \times 10^{14}$ & 125 & 0.05 \\
\hline \multirow[t]{2}{*}{ Niobium } & 873 & $5 \times 10^{20}$ & $1 \times 10^{16}$ & $50-60$ & $0.1-0.2$ \\
\hline & 1173 & $5 \times 10^{20}$ & 0 & & \\
\hline $\mathrm{Nb}-1 \% \mathrm{Zr}$ & 1063 & $2.5 \times 10^{22}$ & $<2 \times 10^{14}$ & 510 & $<1.4$ \\
\hline
\end{tabular}

Fluence for neutrons $>0.1 \mathrm{MeV}$. 
TABLE 5.1-3

\section{REACTOR TRANSMUTATION RATES"}

\begin{tabular}{|c|c|c|c|}
\hline Metal & $\begin{array}{l}\text { Helium } \\
\text { (ppm/yr) }\end{array}$ & $\begin{array}{l}\text { Hydrosen } \\
\text { (ppm/yr) }\end{array}$ & $\begin{array}{l}\text { Solid Product } \\
(\mathbf{( x / y r )} \\
\end{array}$ \\
\hline $\begin{array}{l}\text { Nb CTR Wall } \\
\text { Nb EBR-AI Core } \\
\text { V CTR Wall } \\
\text { V EBR-II Core }\end{array}$ & $\begin{array}{r}270.0 \\
4.0 \\
790.0 \\
3.5\end{array}$ & $\begin{array}{r}890 \\
180 \\
1500 \\
100\end{array}$ & $\begin{array}{l}1.4(\mathrm{Zr}, \mathrm{Mo}, \mathrm{Y})^{\mathrm{a}} \\
\mathrm{b.c} \\
\sim 0.7(\mathrm{Cr}, \mathrm{Ti})^{\mathrm{d}} \\
\mathrm{b}, \mathrm{c}\end{array}$ \\
\hline $\begin{array}{l}{ }^{2} \text { Data from St } \\
\text { neutrons } /\left(\mathrm{cm}^{2} \mathrm{sec}\right) \\
\text { 20-year life Appros }\end{array}$ & $\begin{array}{l}\text { et. } \\
\text { olid proc } \\
\text { lately } 66 \%\end{array}$ & $\begin{array}{l}\text { CTR f } \\
\text { initially } \\
3 \% \text { Mo. }\end{array}$ & $\begin{array}{l}3.7 \times 10^{15} \\
67 r \text {, at end of }\end{array}$ \\
\hline \multicolumn{4}{|c|}{$\begin{array}{l}{ }^{b} \text { Cross sections from Alter and Weber }{ }^{24} \text { Total EBR-ll core flux } \\
\text { taken as } 3 \times 10^{15} \text { neutrons } /\left(\mathrm{cm}^{2} \mathrm{sec}\right), 100 \% \text { plant Sactor assumed. } \\
c_{\text {He production in EBR }=11 \text { would be higher than value given }} \\
\text { because of contribution from nitrogen imputiry content of typical } \\
\mathrm{Nb} \text { or } \mathrm{V} \text { samples. }\end{array}$} \\
\hline
\end{tabular}

reactors, and the ratio of displacement rate to belium production rate is very different from that in fast fission reactors (see Fig. 3.5-9). Such high helium concentrations are known to reduce the ductility of metals sharply at temperatures near half the melting point by promoting intergranular fracture. Gas atoms, especially helium atoms, in a solid are also known to aid the void-nucleation process and enhanced swelling could result. Also, a possibility exists that helium atoms can aggregate to form bubbles and cause additional swelling. However, Martin ${ }^{22}$ has shown that swelling from helium bubbles should not be a serious problem in niobium as long as the helium bubble radius is less than $0.1 \mu \mathrm{m}$. The solid transmutation products may produce slight strengthening from solidsolution effects and may even affect the ductility and swelling of the metal; however, no experimental data are available with which to substantiate this point.

The tritium breeding ratio of the blanket system is influenced by the first wall. Although extensive neutron transport calculations are required th determine whether molybdenum or niobium is best for tritium breeding, preliminary calculations indicate that the higher $(n, 2 n)$ cross section for molybdenum enhances the breeding ratio. The superior high-temperature strength of molybdenum ${ }^{25}$ suggests that a thinner first-wall metal would be adequate, if made from molybdenum. A thinner first wall, derived from strength considerations, is less likely to degrade the neutronic (breeding) characteristics than a thicker niobium first wall. Further, the $(n, \gamma)$ cross sections are more favorable for molybdenum than for niobium, as are the $(n, 2 n)$ cross sections.

5.1.2.3 Chemical Interactions with First Wall. Chemical reaction between the insulator and the refractory metal alloy is discussed in Sec. 5.4.1. Two other possible sourres of significant chemical reactions must be considered. Impurities such as carbon, oxygen, and nitrogen in the molten lithium could exchange with the refractory metal alloy, and thereby embrittle the metal. Molybdenum and niobium differ in their solubilities for these impurities and their embrittlement by them. Another important reaction involving tritium is similar in certain respects and different in others. The molten lithium cooling the first wall will contain tritium in low concentrations (1-10 $\mathrm{ppm}$ ), and tritium will diffuse into the metal. Nobium and vanadium suffer hydrogen embrittlement at and near room temperature; molybdenum, on the other hand, does not suffer hydrogen embrittlement. It is generally believed that hydrogen embrittlement to the first wall during shutdown can be preverized by pumping away the hydrogen isotopes before shutdown. Faber and Schultz ${ }^{26}$ found that hydrogen contamination in niobium following ultrahigh vacuum degassing is very difficult to prevent. The same difficulty should be expected in vanadium. Molybdenum alloys could replace niobium and vanadium if this embrittlement is a serious problem. Current welding and forming practices are more advanced for niobium and vanadium than for molybdenum even though much effort has been applied to molybdenum alloys recently. At present no insurmountable problems seem to exist with these alloys.

\subsection{BLANKET}

The blanket struc:ure will contain molten lithium to breed tritium and to carry away energy deposited by the fusion neutrons. Refractory metal alloys will be used to construct the lithium cooling flow channels. Graphite moderators and beryllium neutron multiplies are used to improve tritiuni breeding. Electrical insulators are used to encapsulate graphite moderator blocks to reduce eddy currents losses. The temperature in the blanket smusture will vary by $\sim 400 \mathrm{~K}$ relative to the coolant inlet temperature. Temperature transients will occur in the blanket and have been estimated in Sec. 3.5.3. Thermal stresses will be generated by these temperature transients. Forces associated with flow surges will be induced by the arrest of the flowing lithium when the compression field is activated, and cause additional stresses in the flow channel wall. The neutron flux will decrease with distance away from the plasma center (Fig. 4.5-5). Near the first wall, the neutron 
flux wiil be at the maximum; near the outside of the blanket edge, the flux will be a factor of $\sim 0.01$ of the first-wall flux. Hence, radiation damage rates will vary widely in structural metal alloys and in insulators.

\subsubsection{Beryllium Region}

Beryllium metal melts at $1550 \mathrm{~K}$, and is an anisotropic, hep metal. Fast neutron irradiation generates helium at high rates. ${ }^{29}$ Poor ductility and high chemical reactivity, especially with oxygen, are adverse properties of beryllium and its alloys. Controlled porosity could be used to keep helium gas pressure in bubbles, but may not be stable under the temperature and radiation-enhanced diffusion conditions in the RTPR.

\subsubsection{Graphite Region}

Manufactured graphites are polycrystalline materials made up of highly anisotropic crystals. These graphites are never free of porosity and internal cracks because of inadequacies of manufacturing techniques and because of the inability of graphite to relax differential contraction stresses except by cracking across crystal boundaries. Preferred orientation is often present in manufactured graphites. Pyrolytic carbons and graphites made by cracking hydrocarbon gases are still another class of carbon materials. Manufactured graphites and pyrolytic carbons have been used extensively in nuclear applications, and much is known about the response of graphites to radiation. Nightingale ${ }^{30}$ has reviewed the high temperature response of manufactured graphites to radiation; Bokros, ${ }^{31}$ Hewetts, ${ }_{1}^{32}$ and Luhleich, ${ }^{33}$ for example, have reported the response of pyrocarbons to radiation. As must be expected for highly anistotropic materials made up of crystals, with or without preferred orientation, the response is complex and varies from material to material. Manufactured graphites expand $>1 \%$ of length to fluences of $\sim 10^{20} \mathrm{n} / \mathrm{cm}^{2}$; contraction then occurs, which is lincar with exposure. Kaal, Stevens, and Bokros ${ }^{34}$ reported the density and dimensional changes induced by fast-neutron irradiation to fluences of $8 \times 10^{21} \mathrm{n} / \mathrm{cm}^{2}$ at temperatures from 900 to $1700 \mathrm{~K}$. Isotropic pyrolytic carbons do not increase in volume at high fluences above $1300 \mathrm{~K}$. Only isotropic pyrolytic carbons have been found to have the dimensional stability required for fuel element application in fission reactors.

Carbon has certain undesirable neutron cross sections which make it very susceptible to radiation damage. The ${ }^{12} \mathrm{C}\left(\mathrm{n}, \mathrm{n}^{\prime}\right) \quad 3 \alpha$ reaction, for example, will generate large quantities of helium ges and this will tend to destroy the graphite moderator. Controlled porosity in the moderator microstructure and venting the moderator will accomodate the gas generation without excessive pressure and stress generation and attendant crumbling. Heat transfer from the moderator to the lithium will involve conduction through the refractory metal alloy can and the insulator. Some thermal stress and thermal shock in the moderator may occur because of the plasma burn pulses, but the graphite is stable to very high temperatures and has excellent resistance to thermal shock and thermal stress. Corrosion of the graphite moderator by molten lithium and by the tritium it contains will be prevented by canning in a refractory metal.

\subsubsection{Structural Metals in Blanket}

The same factors involved in the first wall apply with few differences in the materials in the blanket structure. The reader is directed to Secs. 5.1.2, 5.1.2.1, 5.1.2.2, and 5.1.2.3 for details.

\subsection{COMPRESSION COIL}

The major material problem expected to be of concern in the room temperature compression coil is the increased electrical resistance attendant to the incident radiation flux. The electrical resistance of the copper compression coil will increase by two mechanisms: (1) neutron transmutation of the copper atoms to nickel or zinc, and (2) the creation of lattice defects which accompany an imperfect recombination of vacancy-interstitial pairs. The production rates of nickel and zinc are expected to be $112 \mathrm{ppm}$ and $80 \mathrm{ppm}$ per year, respectively, at the inner radius of the compression coil; these rates decrease by a factor of $\mathbf{3 5}$ to $\mathbf{5 0}$ at the outside surface of the coil. Brick and Phillips ${ }^{35}$ report the electrical resistivity of $\mathrm{Cu}-\mathrm{Ni}$ and $\mathrm{Cu}-\mathrm{Zn}$ alloys as a function of solute concentrations. These data indicate $22.70 \%$ increase in electrical resistance per ycar as 2 res'lt of nickel production and a $0.38 \%$ as 2 result of zinc production for $300 \mathrm{~K}$ at the inner radius.

The production of vacancy-interstitial pairs by fast neutron irradiation in copper has been a topic of extensive research. ${ }^{36,37}$ The excess resistivity at saturation range from 0.34 to $0.33 \mu \Omega-\mathrm{cm}$ at $\sim 300 \mathrm{~K}$. This added resistivity represents a $18 \%$ increase relative to the electrical resistivity of annealled copper at $300 \mathrm{~K}$.

The increase in electrical resistivity of copper which results from the creation of point defects far exceeds that expected for the transmutation of copper atoms to nickel and zinc. The overall increase in resistivity, however, does not appear to present a serious problem. 


\subsection{COOLANT}

\subsubsection{General Problems with Lithium}

The necessity of breeding tritium at a slightly greater rate than it is used as fuel so severely limits the choice of coolants that, coupled with the desire to minimize the blanket thickness (and thus minimize joule losses in the compression coil), the coolant choice is limited to lithium. To further enhance the breeding, the inner portions of the blanket are cooled with natural lithium, while the outermost portion of the blanket is cooled with ${ }^{6} \mathrm{Li}$, which is a more effective breeder of tritium with lowenergy neutrons.

The use of lithium as a coolant in a fusion blanket has been recently reviewed by Maroni, Cairns, and Calfasso. ${ }^{38}$ Maroni et al. give a thorough discussion of the corrosion of blanket materials by lithium. Most of the following is abstracted from the source.

To prevent the spread of radioactivity compounds, a secondary coolant loop is utilized in the plant, with sodium as the heat transfer medium.

\subsubsection{Corrosion Effects by Lithium}

The corrosion of metals by liquid lithium generally has been investigated under poorly defined conditions. Therefore, the majority of corrosion data are useful only as guidelines to the behavior of materials in lithium. Since the effect of impurities (oxygen, nitrogen and carbon, particularly) can have profound effects upon corrosion and penetration rates, disagreement between investigators is the rule rather than the exception. The absence of control of these impurities in many corrosion studies have rendered a major portion of the earlier work questionable.

5.4.2.1 Corrosion of Refractory Metals. Cowles and Pastemak ${ }^{39}$ reviewed the literature up to 1968 , and Maroni, Cairns, and Calfasso ${ }^{38}$ again in: 19:2. Table 5.41 summarizes some of the corrosion resir. dive of refractory metals and alloys to lithium. ${ }^{38}$ Lithium can readily attack and penetrate niobium, when the oxygen content of the metal is excessive. For instance, corrosion of niobium at $900 \mathrm{~K}$ is serious when the oxygen content is above $\sim 400$ ppm. Oxygen in the lithium increases the dissolution rate of niobium, but has little or no effect on the lithium penetration rate.

The problem of lithium penetration due to oxygen contamination has been greatly ameliorated by the use of alloying agents which "getter" oxygen, such as titanium, zirconium, and hafnium, to form alloys such as $\mathrm{Nb}-1 \% \mathrm{Zr}$,
Nb-28\% Ta-10.5\% W-0.9\% Zr (FS-85), and T2-8\% W-2\% $H f(T-111)$. The corrosion resistance of a number of these alloys is given in Table 5.41. To maximize the benefit obtained by the use of oxygen getters, it is necessaty to employ a heat treatment that encourages the reaction between oxygen and zirconium or hafnium before the refractory alloy is exposed to lithium. It has been found that an annealing treatment at $1500-1600 \mathrm{~K}$ for one hour is appropriate for $\mathrm{Nb}-1 \% \mathrm{Zr}, \mathrm{Nb}-28 \% \mathrm{~T}-10.5 \% \mathrm{~W}-0.9 \%$ $\mathrm{Zr}$, and $\mathrm{T} 2-8 \% \mathrm{~W}-2 \% \mathrm{Hf}^{46}$ This treatment results in the combination of sufficient oxygen with the getter to reduce the oxygen concentration below the threshold level, resulting in an alloy with good corrosicn resistance to lithium.

More recently, Sessions and DeVan ${ }^{47}$ investigared the effect of heat treatment and test temperature on the compatibility of several advanced refractory alloys with lithium. Their results, some of which are summarized in Table 5.3-2, showed that (1) attack by lithium at $800 \mathrm{~K}$ was less severe than anticipated on the basis of current corrosion models, (Z) at higher oxygen levels $\$ 2000$ ppm), corrosion at $800 \mathrm{~K}$ was both transcrystalline and intergranular, whereas, attack at $1300 \mathrm{~K}$ was predominantly intergranular, (3) in general, heat treatment at $1600 \mathrm{~K}$ enhanced resistance of the materials tested to corrosion by lithium, and (4) hydrogen pickup during decontamination can be a significant problem with advanced niobium- and tantalum-based alloys when rapid, hydrogen-producing reactions occur.

The nitrogen content of niobium (up to at least $0.1 \%$ ) does not have a great effect on the corrosion rate by lithium. ${ }^{48}$ However, as indicated above the nitrogen content of the lithium does have an influence on the solubility of refractory metals in lithium. Similarly, the carbon content of niobjum (up to at least $0.1 \%$ ) does not influence the corrosion rate by lithium at $1190 \mathrm{~K}$, as might be expected from the fact that niobium carbide is more stable than lithium carbide.

The corrosion resistance of she group VIB metals (cbromium, molybdenum, and tungsten) in lithium has not been as thoroughly investigated as that of niobium, and tantalum, and their alioys, but the same general problems and soiutions obtain. As shown in Table 5.4-1, titanium and zirconium are of ten used as oxygen getters. Alloys rich in molybdenum and tungsten show good corrosion resistarice to lithium at temperatures well above $1300 \mathrm{~K}$, for at least $1000 \mathrm{~h}$, even under flow conditions.

5.4.2.2 Corroxion of Curamic Materials. Although the design of the RTPR presented herein has provided for separation of lithium from any contact with the ceramic insulator, and even double separation from the carbon in 
TABLE 5.4-1

CORROSION RESISTANCE OF SOME REFRACTORY METALS AND ALLOYS TO LTHIUM

\begin{tabular}{|c|c|c|c|}
\hline Sample & Condicions & Results" & Reference \\
\hline $\mathbf{T i}$ & $\begin{array}{l}980-1090 \mathrm{~K}, 1080 \mathrm{hr} \text {, } \\
\text { convection }\end{array}$ & $\begin{array}{l}\text { nil to light } \\
\text { attack }\end{array}$ & 40 \\
\hline $\mathbf{T i}$ & $\begin{array}{l}1273 \mathrm{~K}, 400 \mathrm{hr} \text {, } \\
\text { capsule }\end{array}$ & slight attack & 41 \\
\hline $\mathbf{Z r}$ & $\begin{array}{l}1090-1040 \mathrm{~K}, 1070 \mathrm{hr} \text {, } \\
\text { convection }\end{array}$ & nil attack & 40 \\
\hline $\mathbf{z r}$ & $\begin{array}{l}1273 \mathrm{~K}, 400 \mathrm{hr} \text {, } \\
\text { capsule }\end{array}$ & nil attack & 41 \\
\hline $\mathbf{V}$ & $\begin{array}{l}1090 \mathrm{~K}, 1194 \mathrm{hr} \\
\text { convection }\end{array}$ & nil attack & 40 \\
\hline Nb & $\begin{array}{l}1090-1370 \mathrm{~K}, 8233 \mathrm{hr} \text {, } \\
\text { convection }\end{array}$ & nil attack & 43 \\
\hline $\mathrm{Nb}-1 \% \mathrm{Zr}$ & $\begin{array}{l}1640 \mathrm{~K}, 1000 \mathrm{hr} \text {, } \\
\text { capsule }\end{array}$ & little attack & 42 \\
\hline $\mathbf{N b - i \% Z r}$ & $\begin{array}{l}1420 \mathrm{~K}, 6000 \mathrm{hr} \text {, } \\
\text { capsule }\end{array}$ & slight attack & 44 \\
\hline $\mathrm{Nb}-1 \% \mathrm{Zr}$ & $\begin{array}{l}1030-1600 \mathrm{~K},>20,000 \mathrm{hr} \\
\mathrm{hr} \text {, convection }\end{array}$ & $\begin{array}{l}\text { nil to slight } \\
\text { attack }\end{array}$ & 43 \\
\hline $\mathrm{Nb}-10 \% \mathrm{~W}-1 \% \mathrm{Zr}-0.1 \% \mathrm{C}$ & $\begin{array}{l}1480 \mathrm{~K}, 9437 \mathrm{hr} \text {, } \\
\text { capsule }\end{array}$ & no corrosion & 44 \\
\hline $\mathbf{T a}$ & $\begin{array}{l}1090-1260 \mathrm{~K}, 2261 \mathrm{hr} \text {, } \\
\text { convection }\end{array}$ & nil attack & 43 \\
\hline $\mathbf{C r}$ & $\begin{array}{l}1090 \mathrm{~K}, 100 \mathrm{hr} \text {, } \\
\text { capsule }\end{array}$ & 1 mil dissolution & 45 \\
\hline $\mathbf{T a}$ & $\begin{array}{l}1090-1260 \mathrm{~K}, 2261 \mathrm{hr} \text {, } \\
\text { convection }\end{array}$ & nil attack & 43 \\
\hline Cr & $\begin{array}{l}1090 \mathrm{~K}, 100 \mathrm{hr} \text {, } \\
\text { capsule }\end{array}$ & 1 mil dissolution & 45 \\
\hline Mo & $\begin{array}{l}1640 \mathrm{~K}, 1000 \mathrm{hr} \text {, } \\
\text { capsule }\end{array}$ & little attack & 42 \\
\hline Mo-0.5\%Ti & $\begin{array}{l}1090 \mathrm{~K}, 694 \mathrm{hr} \text {, } \\
\text { convection }\end{array}$ & nil attack & 43 \\
\hline $\begin{array}{c}\text { Mo-0.5\%Ti-0.1\%Zr- } \\
0.01 \% C(T Z M)\end{array}$ & $\begin{array}{l}1920 \mathrm{~K}, 1000 \mathrm{hr} \text {, } \\
\text { capsule }\end{array}$ & no attack & 42 \\
\hline \multicolumn{4}{|l|}{ Mo-50\%Re } \\
\hline $\begin{array}{l}\text { W } \\
\text { Re }\end{array}$ & & & - \\
\hline $\left.\begin{array}{l}\text { W-0.9\%Nb } \\
\text { W-15\%Mo } \\
\text { W-10\%Re } \\
\text { W-25\%Re }\end{array}\right\}$ & $\begin{array}{l}1640 \mathrm{~K}, 1000 \mathrm{hr} \text {, } \\
\text { capsule }\end{array}$ & no attack & 42 \\
\hline
\end{tabular}


TABLE 5.4-2

\section{SUMMARY OF EFFECT OF OXYGEN ADDED AT $1300 \mathrm{~K}$ ON CORROSION OF REFRACTORY ALLOYS IN LITHIUM ${ }^{46}$}

Alloy

D-43

(Nb-10\%W-1\%Zr-0.1\%C)

B-66

(Nb-5\%Mo-5\%V-1\%Zr)

FS-85

(Nb-28\%Ta-10\%W-1\%Zr)

Cb-752

(Nb-10\%W-2.5\%Zr)

Cb-753

(Nb-5\%V-1.25\%Zr)

T-111

(T2-8\%W-2\%Hf)

$\frac{\text { Oxygen Concentration, ppm }}{\text { Before Test }} \frac{\text { After Test }}{\text { Test }}$

990

2000

1800

2700

1200

1900

1400

2500

2000

1500

2500

160

380

780

250

340

290

620

300

730

450

\begin{tabular}{|c|c|}
\hline $\begin{array}{c}\text { Lithium } \\
\text { Concentration } \\
\text { After Test, } \\
\text { ppm } \\
\end{array}$ & $\begin{array}{c}\text { Depth of } \\
\text { Corrocion } \\
\text { Attack, } \\
\mu \mathrm{m} \\
\end{array}$ \\
\hline $\begin{array}{l}\text { b } \\
\text { b }\end{array}$ & $\begin{array}{l}10 \\
30\end{array}$ \\
\hline 39 & 25 \\
\hline 11 & $38-50$ \\
\hline $\begin{array}{r}48 \\
180\end{array}$ & $\begin{array}{c}13-25 \\
38\end{array}$ \\
\hline 36 & $8-13$ \\
\hline$<10$ & $8-13$ \\
\hline b & $13-25$ \\
\hline 68 & 75 \\
\hline 27 & 75 \\
\hline
\end{tabular}

75

the blanket, lithium leaks will be inevitable, and the ceramic used should exhibit at least nominal resistar ce to spontaneous reaction with the lithium. A cursory examination of possible ceramics which are resistant to attack by lithium reveal only a few candidates. The available data is generally only for static corrosion tests, and would not be applicable to a flowing system where significant temperature variations exist throughout the blanket system. For the present purposes, however, it appears that several ceramics would be adequate.

Resulis of screening studies of ceramic materials by Elliot et $21^{+99}$ and Hays et $21^{50}$ are shown in Table 5.4-3. These results are not very different from what might have been expected on the basis of the free energies of reaction with lithium. None of the simple metal oxides are very much more stable than lithia, and all of the commonly used binding agents are less stabie than lithia. The result is that all of the single oxide samples containing unstable binding agents were attacked in the tests shown in Table 5.4-3. Ceramic samples prepared from a mixture of stable oxides (such as $\mathrm{ThO}_{2}-5 \% \quad \mathrm{Y}_{2} \mathrm{O}_{3}$ and $\mathrm{ThO}_{2}-10 \% \mathrm{Y}_{2} \mathrm{O}_{3}$ ) showed good corrosion resistance at $1366 \mathrm{~K}$ for 1000 exposure.

Since lithium carbide and lithium nitride are not very stable, it is appropriate to investigate the corrosion resistance of various carbides and nitrides. The low electrical resistivity of carbides preclude their use as a first wall insulator (with the possible exception of SiC). Very few results are available; $A \mid N$ and $B N$, have been investigated at $1366 \mathrm{~K}$ and show little promise (Table 5.4-3) for resistance to lithium attack.

Double metal oxides are generally more stable than the single oxides from which they are prepared. Admixing, therefore, provides the opportunity to prepare a double oxide from oxides which individually are not quite as stable as $\mathrm{Li}_{2} \mathrm{O}$, the product being more stable than $\mathrm{Li}_{2} \mathrm{O}$. Some double oxides were tested in lithium at $1366 \mathrm{~K}$, as shown in Table 5.4-3 Cracking and flaking were suffered by $\mathrm{CaZrO}_{3}$ after $500 \mathrm{~h}$ of exposure, whereas, $\mathrm{Mg}_{2} \mathrm{AlO}$ disintegrated in $100 \mathrm{~h}$. 
TABLE 5.4-3

STATIC CORROSION RESISTANCE OF SOME CERAMICS TO LITHIUM

\begin{tabular}{|c|c|c|c|c|}
\hline Sample & $\begin{array}{c}\text { Temp., } \\
\text { (K) }\end{array}$ & $\begin{array}{c}\text { Tinne, } \\
\text { (h) }\end{array}$ & Results & Reference \\
\hline BeO & 1366 & 500 & $\begin{array}{l}\text { Significant attack, } \\
\text { Li penetration }\end{array}$ & 49 \\
\hline $\mathbf{M g O}$ & 1366 & 100 & Disinzegrated & 51 \\
\hline $\mathrm{CaO}$ & 1366 & 500 & Significant attack & s1 \\
\hline $\mathrm{Y}_{2} \mathrm{O}_{3}$ & 1366 & 1000 & Slight attack & 49 \\
\hline $\mathrm{Sm}_{2} \mathrm{O}_{3}$ & 1366 & 1000 & Significant attack & 49 \\
\hline $\mathrm{ThO}_{2}$ & 1366 & 1000 & Slight attack & 49 \\
\hline $\mathrm{ThO}_{2}-5 \% \mathrm{Y}_{2} \mathrm{O}_{3}$ & 1366 & 1000 & Very minor attack & 49 \\
\hline $\mathrm{ThO}_{2}-10 \% \mathrm{Y}_{2} \mathrm{O}_{3}$ & 1366 & 1000 & Very minor attack & 49 \\
\hline AIN & 1366 & 1000 & Severe cracking & 49 \\
\hline $\mathbf{B N}$ & 1366 & 100 & Disintegrated & 49 \\
\hline $\mathrm{CaZrO}_{3}$ & 1366 & 500 & Deep cracking and flaking & 50 \\
\hline $\mathrm{BaZrO}_{3}$ & 1366 & 500 & Deep cracking and flaking & 49 \\
\hline $\mathbf{M g A l}_{2} \mathbf{O}_{4}$ & 1366 & 100 & Disintegrated & 49 \\
\hline
\end{tabular}

A number of insulating ceramics were tested by Cairns et $2 .^{52}$ in lithium at $648 \mathrm{~K}$. Although this temperature is lower than the temperatures of interest for RTPR use, the relative corrosion rates of the ceramics may provide some useful guidelines. Cairns note that the purity and manner of prenaration of BeO had a strong influence on its resistance to lithium; similar effects can be expected for other ceramics and have already been noted for BN. The most promising ceramics for resistance to lithium at $648 \mathrm{~K}$ are hot-pressed, high purity $\mathrm{BeO}, \mathrm{Y}_{3} \mathrm{Al}_{5} \mathrm{O}_{12} \mathrm{ThO}_{2}$, $\mathrm{MgO} \cdot \mathrm{Al}_{2} \mathrm{O}_{3} \mathrm{AIN}$, and $\mathrm{Y}_{2} \mathrm{O}_{3}$.

As can be seen from the paucity of verified results on the corrosion of metals, alioys, and electronically insulating ceramics by lithium under well-known purity conditions, a great deal of work remains to be done before the most suitable materials of construction for RTPR application can be unambiguously identified.

5.4.2.3 Corrosion of Graphite. Although the lithiumcarbon-niobium system has not been extensively siudied, it is expected that it will be similar to sodium-carbon with carbide forming metals. Carbon -xposed to sndium will rapidly transfer through the sodium and form carbides with metals in contact with the sodium at temperature in excess of $700 \mathrm{~K}$. Because of the expected behavior with the lithium-carbon-niobium system, ${ }^{53}$ and because such carbide formation with the niobium would be cause for

replacement of the entire system exposed to that part of the lithium flow, two preventive measures have been taken. First, the lithium coolant system has been divided into eight independent systems, each with its own pump, intermediate heat exchanger, and plumbing system. Therefore, should carbon gain access to the lithium, only a fraction of the plant will need replacement. The second prevention is to utilize a double niobium wall between the lithium and carbon in the blanket; two simultaneous leaks must uccur before the lithium is exposed to carbon. If further study of this system shows that such extreme precautions are not justified, some of the niobjum encasement in the blanket structure can be eliminated, enhancing the breeding ratio in the blanket.

\section{REFERENCES}

1. L. C. Inniello, Ed., "Fusion Reactor First Wall Materials," USAEC Report WASH-1 206 (1973).

2. D. B. Watson and W. Heyes, "Time Lags in the Breakdown of $\mathrm{NaCl}$ at High Temperatures," J. Phys. Chem. Solids 31, 2531 (1970).

3. F. L. Ribe, Comp. "Status Report-LASL Controlled Thermofuclear Research Program for the 12-Month Period Ending December 1972," Los Alamos Scientific Laboratory Report LA-5250-PR (June 1973).

4. J. Bunch, Los Alamos Scientific Laboratory private communication June 1973. 
5. F. Skoberne, Comp., "Quarterly Report on the Space Electric Power $R$ and D Program for the Period Ending October 31, 1972," Los Alamos Scientific Laboratory Report LA-5113. PR (November 1972).

6. J. W. Patten, E. D. McClanahan, and J. W. Johnston, "RoomTempera sure Recrystallization in Thick Bias-Sputzered Coppet Deposits," J. Appl. Phys. 42, 4371 (1971).

7. W. H. Reichelt, W. A. Ranken, C. V. Weave:, A. W. Black stock, A. J. Patrick, and M. C. Chaney, "Radiation Induced Damaje to Ceramics in the EBR-II Reactor," presented at the 1970 Thermionic Conversion Specialists Conference, Oct. 26-29, 1970, Miami, FI.

8. G. L. Guthrie, B. Mastel, H. E. Kissinger, and E. R. Bradley, "Absence of Voids in Neutron Irmadiated Sputtered Nickel," J. Nucl. Mater. 37, 343 (1970).

9. R. S. Nelson, "Filling the Voids in the Fast Reactor Technology," New Scientist and Science Journal, 664 (March 25, 1971).

10. A. T. Santhanam, F. L. Yaggee, and S. D. Harkness, "Hightemperature Helium Embrittlement in Vanadium Alloys," HEDL-TME 72-144 (November 1972).

11. D. Kramer, "Heliuta Embrittlement," Fusion Reactor First Wall Materials Meeting, Germantown (January 1972).

12. H. J. Lave, K. D. Class, and M. Guyette, "Irradiation-Induced Biaxial Creep Behavior of Fuel Element Cladding for Fast Reactors," Proc. IAEA Symp. on Radiation Damage in Reactor Materials Vienna (1969).

13. M. F. Ashby, "On Radiation-Enhancied Creep," UKAEA Report HU-DEAP-TR-21, (February 1971)

14. C. G. Collins, J. Moteff and B. A. Chandlet, "Evaluation of the Potential of Selected Alloys for Use as a Fuel Cladding Material in an LMFBR," GEAP-573 (November 1967).

15. E. E. Hoffman and R. W. Hatrison, "The Compatability of Refractory Metals with Liquid Metals," Refractory Metal Alloys-Metallurgy and Tecbnology, Plenum Press, New York (1968).

16. H. Postma, "Engineering and Environmental Aspects of Fusion Power Reactors," Nuclear News (April 1971), p. 57.

17. F. M. Wiffen, "Radiation Damage in CTRs," Proc. Int. Working Sessions on Fusion Reactor Technology, June 28-July 2, 1971, Oak Ridge, Tennessee.

18. J. L. Brimhall, Pacific Northwest Laboratory, private communication, April 1973.

19. B. A, Loomis, Argonne National Laboratory, private communication, April 1973.

20. A. T. Santhanam, Argonne National Laboratory, unpublished results, 1973.

21. R. Carlander, S. D. Harkness and A. T. Santhanam, "Effects of Fast-Neutron Irradiation on Tensile Properties and Swelling Behavior of Vanadium Alloys." Sixth Intl. Symp. on Effects of Radiation on Structural Metals, Los Angeles (June 26-28, 1972).
22. D. G. Martin, "An Ascesument of Some Rediation Damage Effects in the Contoinment Vesel of a Thermonuclear Reactor," B.N.E.S. Nuclear Fusion Reactor Conf., Culham Laboratory, (September 1969) p. 401.

23. D. Steiner, "The Production of Hydrozen nnd Helium in Metals During Reactor Irradiation," Nuclear Appl. and Tech., 9, 83-92 (1970).

24. H. Alter and C. E. Weber, "The Nuclear Performance of Fusion Blankets," J. Nucl. Mater. 16, 68-73 (1965).

25. A. G. Quartell, Ed., Conference on Niobium Tamtalum, Moly bdenum, ard Tungsten, (Elcevier, New Yoik 1961).

26. K. Farber and H. Schultza, "Hydrogen Contemination in Tantalum and Niobium Following UVU-Degassing," Scripta Met. 6. 1065 (1972).

27. G. S. Hanks, Los Alamos Scientific Laboratory, private communication, (1973).

28. R. A. Krakouski, W. V. Green, and W. C. Turner, "LASL' Experience in Welding Molybdenum," LA-UR-73-565 (March 9. 1973).

29. R. Nagasaki, S. Kawasaki, S. Ohashi, Y. Karita, and N. Tusmo "Behavior of He Gas Bubbles in Neutron-Jmadiated Beryllium," Proc IAEA Symp. on Radiation Damage in Reactor Materials,

30. R. E. Nightingale, Nuclear Grapbite, Academic Press, New York (1962).

31. J. C. Bokros, Cbemistry and Plyysics of Carbos, Vol, 5, P. L Walker, Ed. (Mareel Dekker Publ., New York 1969).

32. D. R. Hewette, "Density and Dimensional Changes in Methane and Porpylene Derived Carbon Imdiated to $2 \times 10^{22}$ neutrons $/ \mathrm{cm}^{2}$ at $715^{\circ} \mathrm{C}, "$ Bull. Am. Cer. Soc., 49, 450 (1970).

33. H. L. Luhlerch, L. S. Sutterlin, H. Hover, and H. Nickel, "Determination of the Distribution of Anorphous Particles in Pyrocarbon Coarings by Meens of Cold Oxidation," Fresenius Z. Anal. Chem., 255, 97 (1971).

34. J. L. Kane, D. W. Stevens, and J. C. Bokros, "Dimensioriel Changes Induced in Poorly Crystalline Isomopic Carbons by Irradiation," Carbon, 10, 561 (1972).

35. R. M. Brick and A. P. Phillips, Structure and Properties of Alloys, p. 71 McGraw-Hill Publ. Co., New York, (1949).

36. G. Burger, H. Meissner, and W. Schilling, "The Influence of the Initial Defect Concentration on the Annealing of LowTemperature Irradiated Metals," Phys. Stat. Sol. 4, 267, (1964).

37. J. A. Horak and T. H. Blewitt, "Fast Neutron Irradintion Induced Resistivity in Metals," Phys. Stav. Sol. (A) 4, 721 (1972).

38. V. A. Muroni, R. J. Cairns, and F. A. Cafasco, "A Review of the Chemical, Physical and Thermal Properties of Lithium that are Related to its Use in Fusion Reactors," Argonne National Laboratory Report ANL-8001, March 1973. 
39. J. O. Cowles and A. D. Pasternak, "Lithium Properties Related to Use as a Nuclear Reactor Coolant," Lawrence Livermore Laboratory report UCRL-S0647 (April 1969).

40. M. S. Freed and K. J. Kelly, "Corrosion of Columbium Base and Other Structural Alloys in High-Temperature Lithium," CANEL report PWAC-335.

41. J. F. Cunningham, "Resistance of Metallic Materials to Cor rosion Attack by High-Temperature Lithium," Oak Ridge National Laboratory Report ORNL-CF-51-7-135 (1951).

42. J. A. DeMastry and N. M. Greenauer, "Investigation of HighTemperature Refractory Metals and Alloys for Thermionic Converters," Battelle Memorial Institute Report BMI-AFPLTR-65-29 (1965).

43. J. H. Devan, A. P. Litman, J. R. DeStefano, and C. E. Sesajons, "Lithium and Potasxium Corrosion Studies with Refractory Metals," in Alkali Metal Coolants, p. 675, International Atomic Energy Agenty, Vienna (1967).

44. A. Romano, A. Fleitman, and C. Klamut, "Behavior of Refrectory Metals and Alloys in Boiling Sodium and Other Boiling Alkali Metals," in Alkali Metal Coolants. p. 663. International Atomic Energy Asency, Vienna (1967).

45. E. E. Hoffman, "Corrosion of Materials in Lithium at Elevated Temperatures," Oak Ridge National Laboratory Repurt ORNL-2674 (March 1959).

46. R. W. Harrison, "The Effects of Welding Atmosphere on the Lithium Corrosion of Refractory Alloys," in Comosion by Liquid Metals, p. 217. J. E. Draley and J. R. Weeks, Eds., (Plenum Press, New York, 1970).
47. C. E. Sessions and J. H. Devan, "Effects of Oxyzen, Heat Treatment, and Tex Temperature on the Compatibility of Several Advanced Refractory Alloys with Lithium," Oak Ridge National Laboratory Report ORNL-4430 (April 1971).

48. J. R. DiStefano, "Corrosion of Refractory Metals by Lithium," Oak Ridge National Laboratory Report ORNL-35s1 (1964).

49. D. Elliot, D. Cerini, and L. Hays, "Liquid MHD Power Convetsion," Space Programs Summary No. 37-41, Vol. IV, Jet Propulsion Laboratory, Pusadena, California (June 1966).

50. L. G. Hays and D. O'Connor, "A $2000^{\circ} \mathrm{F}$ Lithium Erosion and Component Performance Experiment," NASA Report No. 32-1150 (1967).

51. D. S. Jesueman, G. D. Roben, A. L. Grunewald, W. L. Fleshman, K. Anderson, and V. P. Calkins, "Preliminary Investizatics of Metallic Elements in Molten Lithium," report NEPA-1465 (1950).

52. E. J. Cairns et al, in "Development of fiigh Enerpy Batteries for Electric Vehicles, Progres Report for the Period July 1970-June 1971," p. 59, Argonne National Leborniary Report ANL-7888 (December 1971).

53. R. P. Elliot, "Constitution of Binary Alloys: First Supplement," (McGraw-Hill, New York, 1965) p. 219. 


\section{ASSEMBLY, OPERATION, AND MAINTENANCE*}

\subsection{INTRODUCTION}

This chapter discusses the aspects of the RTPR that are influenced by assembly/construction, operation and maintenance/repair considerations. Since the plant is is the early conceptual design stage, detailed discussion of procedures is not appropriate. Instead, some of the anticipated problem areas will be indicated to show how they affect the design of the reactor plant.

The first conceptual design of a power plant incorporating a theta-pinch fusion reactor has revealed some of the unique aspects of this kind of plant. This design has taken into account the need for ease of maintenance, simplicity of construction, and remote handling of reactor components.

Three major, distinct, and in some ways unique, phases in the operation of a plant can be identified:

1. Startup (not including preoperational checkout).

2. Normal operation.

3. Maintenance and repair (both on-line and during shutdown).

Some important ways in which maintenance considerations influence the design are:

1. Ease with which faults can be diagnosed and located.

2. Degree to which a system or component can be isolated for maintenance.

3. Accessibility for personnel, tools, and equipment.

Successful maintenance and repair programs begin with the first stages in the design of a plant. It is very difficult to add on adequate maintenance to a plant that has not been designed with the need for repairs and maintenance in view. Of course, the finest quality parts must be obtained; but even these must be maintained. Usually a

-P. V. Dauzvardjs, J. H. Talboy, ANL. plant is designed for normal operation and camnot be started up withour defeating interlocks and perhaps safery features. The design must recognize that the most dangerous plant is the one that is shut down for routine maintenance or repair.

The greatest need for instrumentation exists during the startiup period. That equipment must be easily removable or put in standby status. The operating instruments should be kept to a minimum, and should not include essentially diagnostic hardware-there is no point in maintaining something that is not vital for the day-to-day operation of the plant, or for safety.

Very accurate records and drawings must be kept, together with the reasons for the specifications. Since the designers usually do not operate plants, they must give the operators explicit information on the design rationale for all components so that intelligent repairs and/or replacements can be made, because "exact replacements" are usually not available in a few years.

\subsection{SUMMARY DESCRIPTION}

\subsubsection{General}

During construction and assembly, it must be recognized that those who will operate the plant will need to repair and replace parts and make the modifications that experience will show to be needed. Therefore, during assembly and construction it is imperative that pnotographs of each "layer" of machine be made and preserved. When items such as conduits, cables and pipes are hidden in concrete, accurate instructions for locating them must be generated and filed.

Of course, the remote assembly-disassembly machine and the items with which it interacts must be designed as a unit. The machine should be on hand, and used to actually install all of the modules of the reactor. In this way design fault: will be found and corrected before the structures become radioactive.

\subsubsection{Construction and Assembly of a Module}

As mentioned in the summary description, there are 176, two-meter-long modules in the RTPR. Each module 
blanket consists of 100 radial segments. This number of segmenis was selected so that the size of each one is reasonably large for ease of assembly and to allow a reasonable electrical insulation thickness between segments.

The lithium ducting within the blanket is formed of $0.05 \mathrm{~cm}$-thick $\mathrm{Nb}-1 \% \mathrm{Zr}$ alloy. All external surfaces of the lithium ducting, which is made into one integral piecre, (Fig. 4.5-2), is coated with an $0.03 \mathrm{~mm}$-thick layer of $\mathrm{Al}_{2} \mathrm{O}_{3}$. All surface of the blanket structure that contacts the lithium coolant will be left electrically uninsulated.

The graphite and beryllium portions of the blanket are canned separately in niobium alloy to protect them from porential lithium leakage. The $\mathrm{C}$ and $\mathrm{Be}$ logs are inserted and $\mathrm{t}^{*}$ welded in place. The $\mathrm{Al}_{2} \mathrm{O}_{3}$ insulation is applied via well-established plasma spraying techniques.

The natural lithium is pumped through the blanket segment and a portion exists via a tube passing through the stagnant lithium- 6 region to remove the heat generated. This tube is in turn welded to the natural lithium exit manifold (Fig. 4.5-2).

The 100 radial segments are assembled by being temporarily banded into their final configuration while the lithium coolant manifolds are welded in place. These manifolds support the partially assembled module. $\mathrm{Al}_{2} \mathrm{O}_{3}$ thermal insulation is applied around the outside of the blanket in the form of slabs with overlapping sliding joints to accomodate the thermal expansion of the blanket.

The implosion heating coil (Fig. 4.1-2) is made of potted conductors cooled by a radiation-resistant fluid. These conductors are wound to form a cylindrical shape. Each conductor traverses the length of the cylinder at a pre-determined angle relative to the axis of the cylinder, and then returns to the original end of the cylinder (maintaining the same angle). The electrical leads therefore all enter one end of the coil. Each implosion heating coil is about $0.8 \mathrm{~m}$ long. They will have to be segmented so that they will not be overstressed by thermal expansion.

Compression coils are wound with 1.5-mm-thick copper strips. A $0.95-\mathrm{mm}$ space is left between windings for cooling by a radiation-resistant fluid. Insulators are chosen to be compatible with the anticipated radiation fields. Four compression coils are used per module; two coils are wound clockwise and two coils are wound counterclockwise. One clockwise-wound coil is connected to the adjacent counterclockwise-wound coil.

\subsection{MODULE-HANDLING AND MAINTENANCE}

The overall design philosophy of the reactor arrangement centers on a high availability for the power plant. A large power plant catinot afford long period of down-time without severely taxing reserve power supplies. Therefore, design emphasis has been placed on methods of accelerating and simplifying reactor maintenance. It appears this approach can best be served by a modular reactor concept.

The reactor toroid is formed by 176 modules. Each module (Fig. 3.2-2) is a self-sufficient unit. It is not physically connected to an adjacent module and tharefore can readily be replaced after decoupling fluid and electrical leads.

If the reactor were exposed to air or inert gas when reactor repairs are made, an excessive amount of time would be required to re-evacuate the reactor to the required vacuum level. This problem is overcome by evacuating the reactor maintenance area (reactor upper chamber), thereby allowing module replacement without loss of vacuum. This approach also affords corrosion protection for the refractory alloys present in the lithium systems.

Fue! and ash pump-out is accomplished via a vacuum trough, around the module which forms a duct for pumping the ash from the plasma chamber after passing between the modules. This design negates the necessity for connecting and disconnecting vacuum ducts when replacing modules and also eliminates the need for a continuous vacuum containing toroid with its inherent construction and maintenance problems.

Components of the reactor module are described in more detail in Sections 4.1, 4.2, 4.3, and 4.4 and depicted on Figs. 3.2-1 and 3.2-2.

\subsubsection{Module Lifetime}

Once a module is installed and operating, no maintenance requirements, per se exist. The module, however, must be designed, engineered, and tested to a stringent set of standards that will ensure a predictable lifetime. In this case modules can be replaced on a regular basis before a failure occurs. The functional limit of a module will most probably be determined by damage to the first wall resulting from neutron and thermal effects (see Chapter 4.4.6). More specifically, factors to be considered for the reliable operation of a module are magnetically induced stresses, thermal expansion forces, radiation damage, corrosion, material fatigue, thermal cycling, materials cernpatibility, induced vibration frequencies, "hot spot" elimination, heat transfer, optimum flow path for lithium to reduce magnetic braking, minimization of eddy currents, and electrical insulation materials breakdown.

Each module will be instrumented for temperature and strain measurements in critical areas as well as for 
electrical continuity and cooling fluids pressure. In the event of any detected abnormality, the reactor will not be pulsed again until the abberation is corrected. The fact that the theta-pinch reactor is a pulsed reactor provides a built-in safety feature in that the time between pulses should be sufficient to verify that all systems are functional.

\subsubsection{Module Connections}

Each module is identical and independently hooked up with a lithium inlet and outlet pipe, inlet and outlet coil-cooling lines, fuel-injection tube, compression coil and implosion heating coil, electrical leads and diagnostic leads. Each module has a total of five fluidlines and three electrical connections. The module is suspended from 2 thers. - lly insulated, steel support plug which provides a vace seal when the module is lowered into the vacuum trough. The vacuum trough is formed by the biological shield which, in turn, is lined with a stainless steel sheet that provides a $15 \mathrm{~cm}$ clearance around the module. All module utilities are an integral part of the steel plug and have only to be connested to the main feeds. A secondary vacuum seal is provided above the steel plugs. This seal is made by horizontaliy sliding plates that permit access to the module when verification is received that the chamber above the reactor containing the module-handling machine is evacuated to the proper level. Cooling lines from the module will be connected to their feed lines by a specially designed flange which can be disconnected remotely. Since valves are a prime source of leakage, all valving will be located outside of the biological shield where they can be readily serviced. Whenever possible, cooling lines will be jacketed and the annular space monitored for leakage. Flectrical disconnects will be mechanically clamped.

\subsubsection{Module Failure Modes}

The two principal types of modular failure are anticipited; either coolant leakage or loss of magnetic field. A study is now in progress to detennine the effects of failure of the implosion heating coil or the adiabatic compression coil. It is most probable that a misfire would cause some minor damage to the first wall of the module with the defective coils due to localized heating. Leaking lithium would partially flash-vaporize and become immediately evident in the vacuum fuel-ash purnping system because of increased pumping load, and would also be detected by a mass spectrometer.

Modules with these types of failure must, of course, be identified and replaced. Modules with coolant leaks cannot be gas-purged because this would destroy the reactor vacuum. Rather the defective module must be valved-off and pumped down as far as possible. The vacuum trough will be provided with drain lines to remove any accumulated spillage.

\subsubsection{Module Replacement}

To replace a particular module under a scheduled replacement basis, all cooling lines to the module will be drained by an argon gas purge and then evacuated to a pressure slightly lower than the pressure of the reactor chamber to prevent release of contaminants when lines are disconnected. While proper pressure levels are being achieved, the two handiing machines will be brought into place preparatory to sliding back the secondary vacuum seal plates. One machine will contain the replacement module, and the other will disconnect all lines and leads and retract the defective unit. Upon the satisfactory testing of the replacement cooling lines for gas leakage and electrical checkout, the working fluids will be circulated and the vacuum seal plaies closed. It is conceivable that a module can be replaced in less than an hour.

The level of induced radiation in the module structural materials will preclude personnel from being in the reactor area inside of the biological shield. Therefore, two identical remote "module-handling machines" are provided. The handling machines are suspended from ar. overhead track inside the biological shield and directly over the reactor. These machines, which can also serie as module transport coffins, are remotely operated with radio signal controlled manipulators, hoisting mechanissns and video surveillance. Two machines are provided for redundancy as well as to expedite module exchange. While one machine is loading the defective module, the other is standing by with the replacement module. If the after-heat of the removed module wartants cooling, provision can be made in the machine for pressurized gas cooling. The evacuated upper reactor chamber contaning the module handling machine comnunicates with a module storage area via a rotary air lock: (Fig. 3.1-1).

\subsubsection{Module Storage and Repair}

The storage area located inboard of the upper chamber is under normal atmosphere. The rotary air lick separates the two environments and aliows the hardling machine to pass from one area to the other. Once the handling machine is in the storage area, a transfor mechanism will either unload the "hot" module and place it in a radiation decay area, or load a fresh module from the storige racks into the handing maclsine. An appropriate inventory of 
tested and acceptable modules will always be available from the storage racks for the anticipated replacement schedule. Radioactive modules removed from the reactor that require cooling will be placed in the radiation decay pit located in the center of the building wherein forced gas _..oling will be provided. This pit will also hold any other radioactive materials that require storage. Directly above the storage racks is a "hot cell" complex. Here, modules can be remotely repaired and necessary parts replaced to allow reinsertion of the module into the reactor. Various shops are located outboard of the hot cells and are geared to assemble and test entire modules and module components. The shops include machine, electrical, sheet metal, coil winding, welding, materials testing, instrumentation and assembly.

\subsection{MISCELLANEOUS STARTUP AND MAIN- TENANCE PROBLEMS}

Although the engineering design presented herein is not advanced to the point where a detailed assay of design-related startup and maintenance problems can be made, a number of ger sal problem areas can be identified. The hazards and potential problems related to vacuum technology, liquid-metal technology, helium handling systems, control and instrumentation are well documented from experiences in other areas of science and engineering. In addition to the monumental vacuum requirements, cryogeraic hazards, and liquid-metal problems, the task of operating and maintaining the RTPR is further complicated by the large inventories of tritium and radioactive structure. Furthermore, the high-voltage requirements add another restraint and/or design limitation. All these factors will influence profoundly the ultimate working design for the RTPR, and elucidations of this influence will be the goal of future systems studies of the RTPR. 


\section{APPENDIX A* \\ DERIVATION OF DESIGN RELATIONSHIPS FOR THE IMPLOSION HEATING PROCESS}

\section{A.1 SIMPLE IMPLOSION HEATING MODEL}

The model used to describe the simple implosion heating process is shown in Fig. 2.1-2 and Fig. A-1 with rise-time $\tau_{R} \sim 0$. An ion sheath separates the magnetic field, $B_{S}$, and the plasma advances radially inward at a speed $u_{s}$, projecting ions inward at a speed $2 u_{s}$. The plasma filling density inside this magntic piston is $n_{S}$, and the plasma ahead of the piston is assumed to be cold $(\sim 1 \mathrm{eV}$ in practice). Only ions are assumed to gain energy during the implosion; the electrons remain cold. In practice, some joule heating of the electrons occurs in the

'T. L. Ribe, LASL.
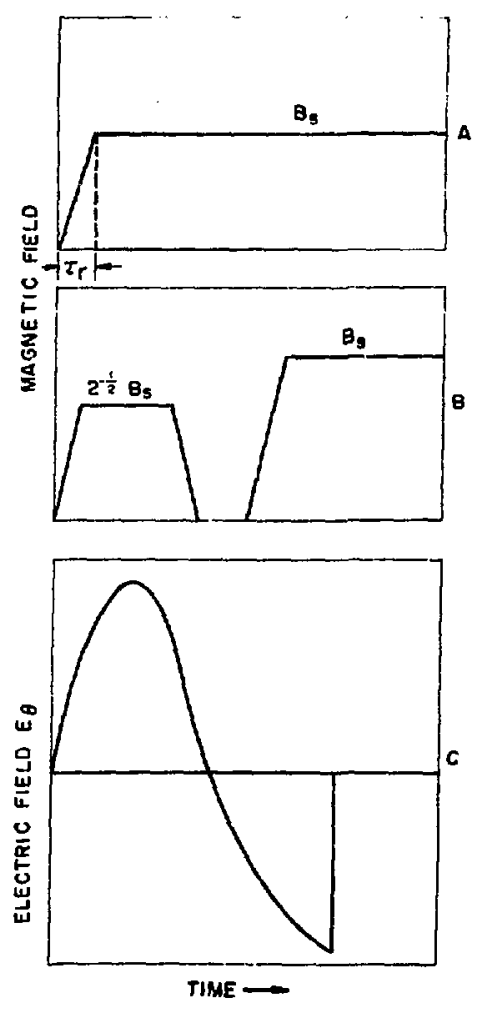

Fig. A-1.

Time-dependence of magnetic field and electric field. sheath. This heating, however, is much less than that of the ions. Plasma-simulation studies' show that the actual process whereby the ions are given a spread of energies from an ideal sheath can be described as one of repeatedly bouncing ions off the sheath wall 25 they pass through the center of the discharge tube. This bouncing leads to oscillations which, on a scale of $0.1 \mu \mathrm{sec}$, settle down to some sheath radius, $\mathrm{bx}_{\mathrm{S}}$. $2 t$ the end of the implosion phase. Here $b$ is the initial radius of the plesma, equal in practice to the inner radius of the first wall $(b=50$ cm).

Simple dynamical considerations show that the rirst ions projected from the sheath will again impinge upon it after passing through the axis of the discharge when the sheath has advanced to $x=1 / 3$. As an average approximation to the actual train of events, all ions inside the sheath at this instant are assumed to thermalize and push the sheath back to $x_{S}$; an amount of work $\left(B_{S}^{2} / 8 \pi\right) \pi b^{2}\left[x_{S}^{2}-(1 / 3)^{2}\right]$ is done against the constant field $B_{S}$, and an ion temperature $T_{S H}$ is obtained. Hence,

$$
\begin{aligned}
& \pi b^{2} n_{S}\left[1-(1 / 3)^{2}\right]\left[1 / 2 m_{1}\left(2 u_{s}\right)^{2}\right] \\
& =\frac{3}{2} \pi b^{2} n_{S} k T_{S H}+1 / 8 b^{2} B_{S}^{2}\left[x_{S}^{2}-(1 / 3)^{2}\right] \\
& (A-1)
\end{aligned}
$$

where $m_{i}$ is the ion mass. by

The momentum of the projected ions is related to $\mathbf{B}_{\mathbf{S}}$

$\mathrm{B}_{\mathrm{S}}^{2} / 8 \pi=2 \mathrm{~m}_{i} \mathrm{n}_{\mathrm{s}} \mathrm{u}_{\mathrm{S}}^{2}$.

After the piston-heated plasma becomes quiescent, pressure balance requires

$\left(n_{S} / x_{S}^{2}\right) k T_{S H}=B_{S}^{2} / 8 \pi$.

Substituting Eqs. (A-2) and (A-3) into (A-1) and eliminating $u_{S}$ and $T_{S H}$ gives

$$
x_{S}=(2 / 5)^{1 / 2}=0.632 \quad(A-4)
$$


Note that by elimination of $B_{S}$ from Eqs. (A-2) and (A-3),

$$
\begin{aligned}
u_{S} & =\left(k T_{S} / 2 m_{1}\right)^{1 / 2} / x_{S} \\
& =2.43 \times 10^{7}\left[k T_{S H}(k e V)\right]^{1 / 2} \cdot(A-5)
\end{aligned}
$$

Here $m_{i}$ is taken as the deuteron mass $\left(m_{i}=3.32 \times 10^{-24}\right.$ $\mathrm{kg}$ ). The initial back emf $\mathrm{V}_{\mathrm{S}}$ (voits) around the inside of the discharge tube is given by

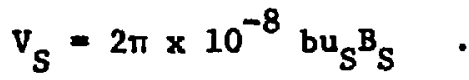

Substituting Eqs. (A-2) and (A-3) into Eq. (A-6) gives

$v_{S}=10^{-8}\left(\frac{\pi}{4 m_{1} n_{S}}\right) b B_{S}^{2}$

$v_{S}=4 \times 10^{-8}\left(\frac{\pi^{3} n_{S}}{m_{1}}\right)^{1 / 2} \frac{b k T_{S H}}{x_{S}^{2}}$

In more convenient units,

$$
\begin{aligned}
& \mathrm{v}_{\mathrm{S}}(\mathrm{kV})=0.58 \times 10^{-6} \mathrm{~b} \mathrm{~B}_{\mathrm{S}}^{2} / \mathrm{P}_{\mathrm{A}}^{1 / 2}(\mathrm{~A}-9) \\
& \mathrm{v}_{\mathrm{S}}(\mathrm{kV})=(4.14) \mathrm{P}_{\mathrm{A}}^{1 / 2} \mathrm{bkT}_{\mathrm{SH}}(\mathrm{keV}),
\end{aligned}
$$

where $p_{A}$ is the filling pressure in $m$ Torr, $b$ is in $c m$, and $\mathbf{B}_{\mathbf{S}}$ is in gauss.

The electric field at the first wall is given by

$$
\begin{aligned}
E_{b}(\mathrm{kV} / \mathrm{cm}) & =V_{S} / 2 \pi \mathrm{b} \\
& =0.65 \mathrm{p}_{\mathrm{A}}^{1 / 2} \mathrm{kT}_{\mathrm{SH}}(\mathrm{keV}) .
\end{aligned}
$$

Substituting Eq. (A-5) into Eq. A-2) gives, for the piston magnetic field,

$$
{ }_{B}(k G)=2.67 \mathrm{p}_{A}^{1 / 2}\left[k_{S H}(\mathrm{keV})\right]^{1 / 2} \cdot(\mathrm{A}-12)
$$

Equation (A-7) shows that the plasma back emf is related quadratically to the coil current, I, driving the field $B_{s}$

$$
B_{s}=0.4 \pi \mathrm{I} / \mathrm{R}_{\mathrm{s}} .
$$

where $\ell_{\mathbf{S}}$ is the length of implosion-heating coil corresponding to I. A plasma impedance, $Z_{p}$, may be defined which corresponds to the time at which $B_{S}$ has risen to its final value and the plasma is at the initial radius $b$. Substituting Eq. (A-13) into Eq. (A-7) yields the following expression for $Z_{p}$ :

$$
z_{p}=v_{S} / I=2 \pi^{3 / 2} \times 10^{-9} m_{1}^{-1 / 2} \frac{b B_{S}}{n_{S}^{1 / 2} \ell_{S}} \text {. }
$$

(A-14)

In practical units, taking $m_{i}$ as the deuteron mass,

$$
z_{p}=0.75 \times 10^{-3} \frac{b_{B}}{p_{A}^{1 / 2} \ell_{S}} \text { (ohms) }
$$

where $b$ and $\ell_{S}$ are in $\mathrm{cm}, P_{A}$ in $m$ Torr, ${ }$ ind gauss. As an example for $b=50 \mathrm{~cm}, B_{S}=14 \mathrm{kG}, P_{A}=17$ mTorr, and $\ell_{S}=100 \mathrm{~cm}: Z_{p}=1.4 \Omega$.

\section{A.2 PROGRAMMED IMPLOSION HEATING; FREE EXPANSION OF IONS}

To outain lower compression for a given $B_{S}$ and $V_{S}$, a magnetic field having the time history shown in Fig. A-1(B) may be used. The initial piston field of magnitude $2^{-1 / 2} B_{S}$ is suddenly removed at time $t_{0}$ when the sheath has arrived at $x=1 / 3$. A larger magnetic field is applied at $3 / 2 t_{0}$, when all ions have advanced to the opposite side of the ion sheath; this field reflects the ions at a velocity 2 $u_{S}$. In contrast to Eq. (A-2), the corresponding momentum balance renders

$$
\mathrm{B}_{\mathrm{S}}^{2} / 8 \pi=4 \mathrm{n}_{0} \mathrm{~m}_{i} \mathrm{u}_{\mathrm{S}}^{2}
$$

Corresponding to Eq. (A-1), the energy balance is now

$$
\begin{aligned}
& \pi b^{2} n_{S}\left[1-(1 / 3)^{2}\right]\left[1 / 2 m_{i}\left(2 u_{S}\right)^{2}\right] \\
& =3 / 2 \pi b^{2} n_{S} k T S H \\
& -1 / 8 b^{2} B_{S}^{2}\left(1-x_{S}^{2}\right) .
\end{aligned}
$$

The last term in Eq. (A-17) represents work done on the plasma after the reversing reflection. All quartities now denote quantities for the case of free expansion. Corresponding to the pressure balance, Eq. (A-3),

$$
\left(n_{s} / x_{S}^{2}\right) k_{S H}=B_{s}^{2} / 8 \pi \text {. }
$$


Solving the three preceding equations for $x_{S}$ yields

$x_{s}=(26 / 45)^{1 / 2}=0.76$.

Equation (A-19) shows the advantage of the programmed field or free expansion in obtaining lower compression ratios after the implosion. The piston velocity is now

$$
\begin{aligned}
u_{S} & =\left(k T_{S H} / 4 m_{1}\right)^{1 / 2} / x_{S} \\
& =1.29 \times 10^{7}\left[\mathrm{kT}_{S H}(\mathrm{keV})\right]^{1 / 2}
\end{aligned}
$$

Since the ions are initially accelerated by the field $2^{-1 / 2} B_{s}$, the expressions for the back emf, electric field, and implosion field become

$$
\begin{gathered}
v_{S}=2^{1 / 2} \pi \times 10^{-8} b_{S^{B}} \\
v_{S}(k V)=0.25 \times 10^{-6}{b B_{S}^{2} / P_{A}^{1 / 2}}^{1 / 2} v_{S}(k v)=1.27 \mathrm{P}_{A}^{1 / 2}{ }_{S H}(k e V) \\
\quad\left(x_{S H}=0.76\right)
\end{gathered}
$$

$B_{S}(k G)=2.22 P_{A}^{1 / 2}\left[k_{S H}(k e V)\right]^{1 / 2} \cdot(A-24)$
These equations show a considerable gain by using the free expansion of the ions. It can be objected that the waveform depicted in Fig. A-1(B) implies very large values of $E_{\theta}$ at time $t_{o}$ and hence is unrealistic. Results of simulation computations which use the $\mathrm{E}_{\theta}$ waveform of Fig. A-1(C), however, show conveniently small values of $\mathrm{E}_{\boldsymbol{\theta}}$ and give a corresponding $\mathrm{B}_{S}$ waveform which performs the same essential functions as that of Fig. A-1(B). These simulation computations give a value of $x_{S}$ that is even larger than the $x_{S}$ derive: from the $B_{S}$ wave form shown on Fig. A-1(B). Therefore, the inference is made that Egs. (A-20) to (A-23) are sufficiently representative of the advantages of free expansion.

Equations $(A-20)$-(A-23) represent the scaling relationships for the implosion heating stage of the RTPR power cycle. These expressions are used in conjunction with the adiabatic relationships in Section 4.2 to develop a complete set of design equations. Table 2.4-1 summaries RTPR parameters which have pertinence to the implosion heating stage and has been derived from these scaling equations.

\section{REFERENCES}

1. J. P. Freidberg, R. L. Morse, F. L. Ribe, Texas Symp. on the Technol. of Controlled Fusion Experiments and Engineering Aspects of Fusion Reactors, Session 5, paper 1, November 20-22, 1972. 


\section{APPENDIX B* COMPUTER OUTPUT SUMMARY OF RTPR PARAMETER DESIGN VALUES}

\section{B.1 GENERAL DESCRIPTION}

Approximately 700 design parameters have been developed in this design study. These data include a wide variety of structural dimensions, system parameters. and performance parameters. Ready access to these data is essential to a flexible and comprehensive systems study. As design development proceeds, it will become even more essential that the expanding data base be readily accessible modifiable.

The need for a workable date management system is appropriately met by a computer used to store data, to present data in a useful form at request, and to modify data quickly and easily upon command. A useful and inexpensive system to perform these functions is the time-sharing option avilable on computers such as the IBM 360 system at ANL and the CDC 7600 system at LASL. Data stored on disks at the computer are recalled on demand at a visual display terminal. Printed output is readily available. Punched card output is also available, but serves primarily as a permanent data record if data stored in the computer are inadvertently lost.

\section{B.2 POTENTIAL OF SYSTEM}

At the minimum level described above, computer management of data represents a high-speed, ready-access storage and typing system. This function alone is adequate justification for the system. The utility of computer management of data increases greatly when the full potential of the system is considered. For instance, a computer program which represents functional relationships between sets of parameters within a system can use the data base as input to generate a new or revised data base. In this way the introduction of a revised input data can generate programmed changes in output data.

The dynamic simulation of the entire fusion reactor power plant represents another application of the proposed computerized design. Mathematical models would represent all individual reactor systems and coupling between systems. This approach could determine a unique set of self-consistent reactor parameters which have been optimized with respect to a selected criterion. The model would draw upon a limited data base as the source of input parameters for the reactor systems as required; all other parameters would then be obtained in 2 selfconsistent manner from a large set of simultaneous equations which would be temporarily and spatially dependent. Output data could be presented in graphical form as desired.

\section{B.3 DESCRIPTION OF EXISTING RTPR SYSTEM}

The present theta-pinch data base consists of two spearate data composites, each having a unique function according to the type of output desired. The two output modes presently available are (1) recall of any desired data set for revision or for addition of new data sets, and (2) a complete sequential listing of all data on each separate reactor system. The following compilation represents the mode (2) output and numerically represents the present status of the RTPR engineering design.

\footnotetext{
•C. B. Dennis, A. J. Hatch, ANL.
} 


\section{B.4 DESIGN DATA FOR REFERENCE THETA-PINCH REACTOR (RTPR)}

\section{RTPR Systems}

1. Implosion (shock) heating systen:

2. Adiabatic compression system

3. Magnetic energy transfer system

4. First wall system

5. Blanket system

6. Biological shielding system

7. Fuel supply/ash removal system

8. Fuel processing system

9. Coolant processing system

10. Energy conversion system

11. Alternate potassium topping energy conversion system

\section{IMPLOSION (SHOCK) HEATING SYSTEM}

1. Plasma parameters at start of burn

2. Implosion heating coil (IHC) parameters

3. Design requirements

\section{Plasma Parameters at Start of Burn}

1. Compression field, kG

2. Compression ratio at start of burn

3. Radius of first wall, $\mathrm{cm}$

4. Electron and ion temperature at start of burn, keV

5. Plasma radius at start of burn, $\mathrm{cm}$

6. Temperature of ions after implosion heating (before equilibration), keV

7. Electron and ion temperature after implosion heating (after equilibration), keV

8. Implosion heating field, $\mathbf{k G}$

9. Implosion velocity, $\mathrm{cm} / \mu \mathrm{s}$

10. Plasma back EMF, kV

11. Implosion heating electric field, $\mathrm{kV} / \mathrm{cm}$

12. Ambient filling pressure $(300 \mathrm{~K}), \mathrm{m}$ Torr

13. Ion density at start of burn, ions $/ \mathrm{cm}^{3}$

14. Ratio of plasma to magnetic pressure 
2. Implosion Heating Coil (IHC) Barameters

1. Mean radius of $\mathrm{IHC}, \mathrm{cm} \quad 90$

2. Length of IHC. $\mathrm{cm} \quad 100$

3. Thickness of $\mathrm{IHC}$ winding, $\mathrm{cm}$

4. Filling fraction for IHC $\quad 0.7$

5. Implosion heating field, $\mathrm{kG} \quad 13.8$

6. Voltage of the lHC capacitor, keV 60

7. Total azimuthal voltage around IHC, keV 650

\section{Dexips Requirements}

1. Implosion heating fjeld, $\mathrm{kG} \quad 13.8$

2. Time for field, $\mathrm{ms} \quad 10$

3. Risetime, microsec 0.1

4. Energy required by the implosion heating stage, MJ/m 1.66

\section{ADIABATIC COMHRESSION SYSTEM}

1. Design requirements

2. Sechanical stresses in the compression coil

3. Compression coil cooling parameters

\section{Dexipn Requirements}

1. Coil winding thickness, $\mathrm{mm}$

2. Number of turns in compression coil 187

3. Width of compression coil, $m \quad 0.5$

4. Neutron heating in coil, $\mathrm{MJ} / \mathrm{m} \quad 0.57$

5. Gamma-ray heating in coil, $M J / m$

6. Total energy generated in coil, $\mathrm{MJ} / \mathrm{m} \quad 11.6$

7. Increase in temperature of copper coil, $\mathrm{K} / \mathrm{pulse} \quad 2.0$

8. Energy necessiry to remove from coil (for 210 sec duty cycle), MW/m

9. Magnesic field requirements:

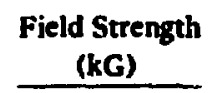

13.8-110

110

110-32.2

$32.2-0$

$\mathbf{0}$
Risetime*
(ms)

20

20

1000
Duration*

(ms)

80

$\sim 8000$

"Nominal values used (see footnote on page fi). 
2. Mechanical Strewes in the Compression Coil

1. Adiabatic compression field, $k G$

110

2. Outward pressure exerted by field on compression coil, MPa

kpsi

3. Average hoop stress, MPa

\section{Comprewion Coil Cooling Parameters}

1. Neutron heating, $M J / m$

2. Gamma-ray heating, $M J / m$

3. Transport current joule heating, $M J / m$

4. Eddy-cument joule heating, $\mathrm{MJ} / \mathrm{m}$

5. Lengeth of compression coil, $m$

0.5

6. Inner diameter of compreasion coil, $m$

7. Outer diameter of compression coil, $m$

8. Conductor (tum) thickness, mm

9. Nur:ber of turns

10. Current per tum, kA

11. Insulation thickness, $\mathbf{m m}$

12. Insulation thickness (glass), mm

13. Mean coil temperature, $C$

14. Coolant inlet temperature, $C \quad 20$

15. Coolant outlet temperature, $C \quad 23$

16. Mean heat transfer coefficient, BTU $\mathrm{hr} \mathrm{ft}^{2}{ }^{\circ} \mathrm{F}$

17. Average temperature rise in coil per pulse, $\mathrm{C}$

18. Weight of copper, $\mathrm{kg} /$ module

19. Weight of coolant per module, kg

20. Coolunt pressure drop through coil, psi

21. Total coclant pressure drop, psi

\section{MAGNETIC ENERGY TRANSFER SYSTEM}

1. Design parameters for 2 superconducting energy tranfer module

1. Dexign Parameters for a Superconducting Every Transfer Module

1. Coupling coefficient, $k_{T}^{2}$

2. Coupling coefficient, $k_{m}^{\frac{1}{2}}$

3. Transfer efficiency, $\%$

4. Field strength of coil $1, k G$

5. Total magnetic energy stored, $M$ J

6. Energy transferred to load, M]

7. Outer coil radius, $\mathrm{cm}$

6. Inner coil radius, $\mathrm{cm}$

9. Intermediate coil radius, cm 
10. Ampere turns, $A$

11. Ampere turns, $A$

12. Ampere turns, $A$

$1.41 \mathrm{E}+07$

13. Current density in superconductor, $A / \mathrm{cm}^{2}$

$1.0 \mathrm{E}+05$

14. Coil thickness, $\mathrm{mm}$

7.16

15. Outer coil thickness, $\mathbf{m m}$

7.16

16. Intermediate coil thickness, $\mathrm{mm}$

17. Inner superconductor volume, $\mathrm{cm}^{3}$

18. Outer superconductor volume, $\mathrm{cm}^{3}$

19. Intermediate superconductor volume, $\mathrm{cm}^{3}$

20. Moment of inertic of inner superconductor winding, $\mathrm{kg} / \mathrm{m}^{2}$

$1.23 E+05$

21. Moment of inertiz of outer superconductor winding, $\mathrm{kg} / \mathrm{m}^{2}$

22. Moment of inertia of intermediate superconductor winding, $\mathrm{kg} / \mathrm{m}^{2}$

23. Refrigerator power, MWe

\section{FIRST WALL SYSTEM}

1. First wall thermal and radiation flux loading

2. Parameters used in first wall heat transfer calculations

\section{First Wall Thermal and Radiation Flux Loading}

14-MEV neutron loading, $\mathrm{MW} / \mathrm{m}^{2}$

Burn time, sec

Cycle time, sec

Radius of the first wall, $\mathrm{cm}$
2.0

0.080

10.0

50.0

8.91E + 13

$1.10 \mathrm{E}+16$

$9.03 E+14$

$2.84 \mathrm{E}+21$

$8.91 E+14$

156.3

63.5

23.6

183.4

71.62

1432.4

286.5

1652.2

493.5 
1. Inside radius of niobium wall, $\mathrm{cm}$

50.00

2. Thickness of alumina insulator, $\mathrm{cm}$

0.030

3. Thickness of niobium wall, $\mathrm{cm}$

0.100

4. Thickness of lithium, $\mathrm{cm}$

10.00

5. Neutron energy deposited in blanket, $M J / m$

93.1

6. Bremsstrahlung energy during burn, $M \mathrm{~J} / \mathrm{m}$

2.25

7. Internal energy of plasma at the time of cooling, $M J / m$

4.13

8. Heat capacity of alumina, J/gK

$0.4184 \mathrm{~T}^{0.18}$

9. Heat capacity of niobium, J/gK

0.335

10. Heat capacity of lithium, J/gK

11. Thermal conductivity of alumina, $W / \mathrm{cmK}$

12. Thermal conductivity of niobium, $W / \mathrm{cmK}$

4.184

13. Thermal conductivity of lithium, $W / \mathrm{cmK}$

$390.0 T^{1.20}$

0.700

0.500

14. Density of alumina, $\mathrm{g} / \mathrm{cm}^{3}$

15. Density of niobium, $\mathrm{g} / \mathrm{cm}^{3}$

3.80

8.55

16. Density of lithium, $\mathrm{g} / \mathrm{cm}^{3}$

0.43

\section{BLANKET SYSTEM}

1. Position and thickness of components in blanket and shield

2. Blanket parameters: area, volume, density, weight

3. Surface areas per $2-m$ segment

1. Position and Thickness of Components in Blanket and Shield

\begin{tabular}{|c|c|c|c|}
\hline Layer & $\begin{array}{l}\text { Inner Radius } \\
\text { (cm) } \\
\end{array}$ & $\begin{array}{l}\text { Outer Radius } \\
(\mathrm{cm})\end{array}$ & $\begin{array}{l}\text { Thickness } \\
\text { (cm) }\end{array}$ \\
\hline 1. First wall & 50.0 & 50.1 & 0.1 \\
\hline 2. Lithium & 50.1 & 53.1 & 3.0 \\
\hline 3. Beryllium & $53.2^{*}$ & 55.7 & 2.5 \\
\hline 4. Lithium & $55.8^{*}$ & 60.3 & 4.5 \\
\hline 5. Graphite & $60.4^{* *}$ & 64.9 & 4.5 \\
\hline 6. Lithium & $65.0^{* *}$ & 67.7 & 2.7 \\
\hline 7. Graphite & $67.8^{* *}$ & 82.6 & 14.9 \\
\hline 8. Enriched ${ }^{6} \mathrm{Li}$ & $82.7^{* *}$ & 88.9 & 6.2 \\
\hline 9. Alumina + Vacuum & $89.0^{*}$ & 89.5 & 0.5 \\
\hline 10. Implosion coil & 89.5 & 90.5 & 1.0 \\
\hline 11. Alumina + vacuum & 90.5 & 93.5 & 3.0 \\
\hline 12. S.S. ring & 93.5 & 93.5 & 0.01 \\
\hline 13. Compression coil & 93.5 & 133.5 & 40.0 \\
\hline 14. Titanium & 133.5 & 143.5 & 10.0 \\
\hline 15. Vacuum & 143.5 & 150.0 & 6.5 \\
\hline 16. Biological shield & 150.0 & Variable & $>200.0$ \\
\hline
\end{tabular}

* Indicates allowance for thickness of $\mathrm{Nb}$ wall.

* Indicates allowance for thickness of $\mathrm{Nb}$-alumina-Nb wall. 
2. Blenket Parameters: Area, Volume, Density, Weight per Module

\begin{tabular}{|c|c|c|c|c|}
\hline Layer & $\begin{array}{l}\text { Cross Sec- } \\
\text { tional Area } \\
\left(\mathrm{cm}^{2}\right) \\
\end{array}$ & $\begin{array}{l}\text { Volume } \\
\left(\mathrm{cm}^{3}\right)\end{array}$ & $\begin{array}{l}\text { Dencity } \\
\left(1 / \mathrm{cm}^{3}\right)\end{array}$ & $\begin{array}{c}\text { Weight } \\
\text { (ke) }\end{array}$ \\
\hline 1. Lithium (1) & 894 & 178,800 & 0.43 & 77 \\
\hline 2. Beryllium & 790 & 158,000 & 1.85 & 292 \\
\hline 3. Lithjum (2) & 1521 & 304,200 & 0.43 & 131 \\
\hline 4. Graphite & 1601 & 320,200 & 2.26 & 723 \\
\hline 5. Lithium (3) & 977 & 195,000 & 0.43 & 84 \\
\hline 6. Graphite & 1957 & $1,391,400$ & 2.26 & 3150 \\
\hline 7. Lithium 6 & 3234 & 646,800 & 0.43 & 278 \\
\hline 8. Insulator & 3306 & 661,200 & 3.80 & 2520 \\
\hline 9. Niobium & & 250,000 & 8.58 & 2180 \\
\hline
\end{tabular}

3. Surface Areas per Module

1. Niobium segment walls, $\mathrm{m}^{2}$

2. Niobium dividers, $m^{2}$

3. Alumina wall coating, $\mathrm{m}^{2}$

4. Alumina divider coatings, $\mathrm{m}^{2}$

Length of niobium welds, $m$

\section{BIOLOGICAL SHIELDING SYSTEM}

1. Reactor module assembly

1. Reactor Module Assembly

1. Thickness of concrete shield, $m$

2. Neutron flux at inner surface of shield, $n / s \mathrm{~cm}^{2}$

3. Neutron flux at outer surface of shield, $n / 5 \mathrm{~cm}^{2}$

4. Photon flux at inner surface of shield, gammas/s $\mathrm{cm}^{2}$

5. Photon flux at outer surface of shield, gammas/s $\mathrm{cm}^{2}$

6. Biological dose rate at outer surface of shield, $\mathrm{mrem} / \mathrm{h}$

2.3

7. Maximum permissible dose rate for free access areas, $\mathrm{mrem} / \mathrm{h}$

0.02

8. Maximum radiation heating rate in concrete shield. $W / g$

0.08

5.7E - on 


\section{FUEL SUPPLYIASH REMOVAL SYSTEM}

1. Design parumeters

2. Vacuum pumping system

1. Desion Parameten (per Module)

1. Pressure of D-T mixture at $810 \mathrm{~K}$, mitorr 46

2. Temperature of gas, ${ }^{\circ} \mathrm{K} \quad 810$

3. Filling pressure at $300 \mathrm{~K}$, mTorr 17

4. Filling pressure at $810 \mathrm{~K}$, mTorr 46

5. Volume of 50-em-radius chamber which must be filled, liters, 1500

6. Required flow rate, Torr-liters/s 250

7. Pumping speed in chamber, liters/s $\quad 14,000$

$\begin{array}{lr}\text { 8. Residual helium, arom percent } & 0.6\end{array}$

2. Vacuum Pumping Syatem

1. Number of first-stage vacuum pumps (19 kW) 352

2. Number of second-stage vacuum pumps (15 kW) 352

3. Number of other vacuum pumps (16 kW) 20

4. Total power requirement for vacuum pumps (MWe) 12.3

\section{FUEL PROCESSING SYSTEM}

1. Design parameters

1. Dexigr Parameters

1. Fuel ash flow, g/hr of gas

10,000

2. Molar composition of gas, $\%$
A) Deuterium
B) Tritium
C) Helium
D) Protium

3. Mean temperature of gas, $\mathrm{K}$

4. Mixture equilibration point, $K$

5. Number of plates in each distillation column

6. Space between columns, in.

7. Diameter of column 1, in.

8. Dismeter of column 2, in.

9. Height of columns 1 and 2, ft

10. Added diameter due to insulator, in.

11. Plate efficiency, $*$

12. Relative volatility of H-D compared to H-T

49
49
1
$<1$
800
50
60
2.5
6
3
15
2.4
30
1.4


1. Design requirements

1. Design Requirements

1. Partial pressure of tritium in lithium, Torr

$<1.0 E-10$

2. Temperature of lithium, $C$

3. Number of salt contactors ( 10 s cycle)

4. Contactor diameter $\mathrm{cm}$

5. Contactor height $\mathrm{cm}$

6. Contactor powet MW ( 10 s cycle)

10. ENERGY CONVERSION SYSTEM

1. Surge tanks

2. Primary lithium pumps

3. Secondary sodium pumps

4. Intermediate heat exchanger

5. Intermediate heat exchanger heat transfer data

6. Steam generator

7. Steam cycle farameters

8. Power plant auxiliary power

9. Power summary

1. Sunge Tanks

1. Number of surge tanks

2. Flow velocity, ft/s

3. Capacity $\mathrm{ft}^{3}$

2. Primary Lithium Pumpa, $\tau_{c}=3 \mathrm{~s}, \eta_{\mathrm{TH}}=40 \%$

1. Number of pumps

8

2. Pump stages

3. Pump type

4. Flow rate, lb/s

Single

Centrifugal

6250

gpm

103,500

5. Head, fx Li

160

psi

30

6. NPSH (operating) ft $\mathrm{Li}$

80.5

(Limit)

50

7. Speed, rpm

450

8. Port size, intake diameter, in.

Outlet dianeter, in.

48

9. Power required, hp 
3. Secondary Sodium Pumps, $r_{c}=3 s, \eta_{\mathrm{TH}}=40 \%$

1. Number of pumps

2. Pump stages

3. Pump type

4. Flow rate, $l b / s$

5. $\mathrm{gpm}$

Bpm

5. Head, ft NA

psi

6. NPSH, ft NA

psi

7. Speed, rpm

8. Port size, intake diameter, in. Outlet diameter, in.

8

Single

Centrifugal

14,800

122,000

115

43.3

92

34

811

48

34

3820

2.85

4. Intermediate Heat Exchanger, $\tau_{c}=3 \mathrm{~s}, \eta_{\mathrm{TH}}=40 \%$

Parameter

Shell

Lithium

1004

775

29

17

6250

5

42.5

168

3.5

36

1800

3100

$36(2)$

36 (2)

300,000
Tubes

Sodium

675

971

100

88

14,800

11.3

32

0.75

0.042

24.6

56,520

9000

1780

$48(1)$

$34(2)$

106,000
17. Weight, lb

5. Intermediate Heat Exchanger Heat Transfer Data, $\tau_{c}=3 s, \eta_{\mathrm{TK}}=40 \%$

1. Number of heat exchangers

8

2. Power rating, MWT (each)

1500

91.42

4. Heat transfer area, sq ft

56,520

1400

5. Overall heat transfer coefficient, BTu/h $\cdot s q$ ft $\cdot F$

63

6. Log mean temperature difference, $F$

Niobium

7. Material (alloy)

500,000 
6. Steam Generator, $\tau_{c}=3 s, \eta_{\mathrm{TH}}=40 \%$

Parameter
1. Number of steam generators
2. Fluid
3. Inlet temperature, $F$
4. Outlet temperature, $F$
5. Inlet pressure, psia
6. Outlet pressure, psia
7. Flow rate, lb/s
8. Power rating, $M W t$
9. Flow speed, ft/s
10. Length, ft
11., Diameter, in.
12. Thickness, in.
13. Material
14. Surface area, sq $f t$
15. Number of tubes
16. Holdup, cu $\mathrm{ft}$
17. Intake diameter, in.
18. Outlet diameter, in.
19. Weight, lb

Shell

B

Sodium

965

650

32

17

14,800

1500

1.42

74.6

180

2.5

304SS

3532

8597

$30(2)$

$30(2)$

400,000
Tubes

Steam
500
903
2660
2435
1560
1500
142.6
0.5
0.065
INCO800
67,275
3600
552
$8(6)$
$12(6)$
200,000

Steam

903

2660

2435

1560

42.6

0.5

600

8 (6)

0

7. Steam Cycle Parameters, $\tau_{\mathrm{c}}=3 \mathrm{~s}, \eta_{\mathrm{TH}}=40 \%$

Fou: Turbine-Generator Type: Tandem-Compound Quadruple Flow

1. Heat input, MWt

3000

2. Steam flow rate, $\mathrm{lb} / \mathrm{s}$

3134

3. Temperature at throttle, F

900

4. Pressure at throtle, psig

2400

5. Gross power output, MWe

1275

6. Gross thermal efficiency, $\%$

7. Number of condenser units

8. Number of feedwater heaters

9. Condenser steam pressure, in. $\mathbf{H g}$

10. Condenser heat transfer surface, sq ft/unit

11. Feedwater pump power, MW

12. Condenser cooling water pump power, $M W$

13. Condenser cooling water inlet temperature $F$ 
8. Power Plant Auxiliary Power, $\tau_{c}=3 \mathrm{~s}, \eta_{\mathrm{TH}}=40 \%$

Subsystem

1. Helium liquefiers for S.-C. energy transfer modules

2. Fuel/ash system:

1st stage vacuum pumps, 352 at $20 \mathrm{~kW}$

2nd stage vacuum pumps, 352 at $16 \mathrm{~kW}$

Tunnel pumps, 20 at $16 \mathrm{~kW}$

Compressors, fuel/ash, compressor, interstage, compressor, to storage, compressor, inert gas cooling, 10 at $40 \mathrm{~kW}$

3. Coolant processing system:

Molten salt pumps, 200 at $1 \mathrm{~kW}$

Contactor drive, 200 at $20 \mathrm{~kW}$

4. Implosion heating and compression coils:

Cooling pumps

Ohmic loss in busbars ( $6000 \mathrm{ft}$ per module, 2 -in. sq busbars)
(MW)

600

600

7.2

5.8

0.3

0.4

0.2

10

16

120

136

14.8

22.8

149

13.2

8.0

207.8

9. Power Summary

Total auxiliary power, MW

968.2

Gross electric power, $M W$

Net electric power, $M W$

11. POTASSIUM TOPPING ENERGY CONVERSION SYSTEM, $\tau_{c}=10 \mathrm{~s}, \eta_{\mathrm{TH}}=56.4 \%$

1. System parameters

2. Potessium boilers

3. Major lithium piping runs

4. Major potassium piping runs 
1. System Parameters $\tau_{\mathrm{c}}=10 \mathrm{~s}, \eta_{\mathrm{gh}}=56.4 \%$

1. Thermal power, MWt

2. Lithium temperature, in/out, $K$

3600

3. Lichium pressure, in/out, psi

$1273 / 1400$

4. Lithium flow rate, $\mathrm{kg} / \mathrm{h}$

$30 / 25$

5. Lithium pump, hp

6. Potassium temperature from primary heat exchanger, $K$

$25 E+06$

$0.4 \mathrm{E}+06$

7. Potassium temperature from turbine, $K$

1255

8. Potassium temperature from feed heater, $K$

866

9. Potassium pressure from primary heat exchanger, atmospheres

1190

10. Potassium pressure from turbine, atmospheres

11. Enthalpy rise in boiler, BTU/Hb

12. Potassium flow rate, $\mathrm{kg} / \mathrm{s}$

1950

2. Potassium Boilers

1. Height, ft

2. Diameter, $\mathrm{ft}$

3. Tube diameter, in.

4. Mean temperature difference, $F$

5. Heat transfer coefficient, $B T u / s q ~ f t \cdot h \cdot F$

6. Number of tubes per boiler

7. Tube wall thickness, in.

8. Weight of tubes, $1 \mathrm{~b}$

9. Weight of shell, $1 \mathrm{~b}$

\section{0}

6

1

5

2660

910

0.042

27,000

50,000

\section{Major Lithium Piping Runs}

\section{Parameter}

1. Temperature, $F$

2. Pressure, psig

3. Density, lb/cu ft

4. Flow rate, lb/s

5. Velocity, $\mathrm{ft} / \mathrm{s}$

6. Length, $\mathrm{ft}$

7. Diameter, in.

8. Thickness, in.

9. Sectional area, sq ft

10. Surface area, sq dt

11. Weight/pipe, tons

12. Pressure loss, psi

13. Number of pipes

Tozal weight, tons

\begin{tabular}{|c|c|c|c|}
\hline \multicolumn{4}{|c|}{ Location } \\
\hline $\begin{array}{c}\text { Module } \\
\text { to } \\
\text { Manifold }\end{array}$ & $\begin{array}{c}\text { Outlet } \\
\text { Manifold }\end{array}$ & $\begin{array}{c}\text { Menifold } \\
\text { to } \\
\text { K Boiler }\end{array}$ & $\begin{array}{c}\text { K Boiler } \\
\text { to } \\
\text { Contactor }\end{array}$ \\
\hline 2050 & 2050 & 2050 & 1832 \\
\hline 17 & 14 & 14 & 2 \\
\hline 25 & 25 & 25 & 27 \\
\hline 85.2 & 1560 & 3750 & 3750 \\
\hline 17 & 9 & 2 & 1 \\
\hline 50 & 290 & 130 & 75 \\
\hline 6 & 36 & 48 & 48 \\
\hline 0.085 & 0.25 & 0.5 & 0.25 \\
\hline 0.2 & 7.06 & 12.5 & 12.5 \\
\hline 78.5 & 2730 & 1650 & 960 \\
\hline 0.15 & 0.15 & 17.5 & 6 \\
\hline 1 & 0.1 & 0.3 & 0.2 \\
\hline 176 & 4 & 2 & 2 \\
\hline 26 & 60 & 35 & 12 \\
\hline
\end{tabular}


Location

Parameter

1. Temperature, $F$

2. Pressure, psig

3. Density

4. Flow rate, Ib/s

5. Velocity, $\mathrm{ft} / \mathrm{s}$

i). Length, ft

7 . Diameier, in.

6. Tinickness, in.

9. Sectional area, sq ft

i0. Surface area, sq ft

11. Weight, tons

12. Pressure loss, psi

13. Number of pipes

Total weight, tons

Total weight of lithium piping $=\mathbf{2 3 8}$ tons

\section{Major Potassium Piping Runs}

Parameter

1. Temperature, $F$

2. Pressure, psia

3. Density, lb/cu ft

4. Flow rate, lb/s

5. Velocity, ft/s

6. Length, $\mathrm{ft}$

7. Diameter, ft

8. Thickness, in.

9. Sectional area, sq ft

10. Surface area, sq ft

11. Weight, tons

12. Pressure loss, psi

13. Number of pipes

Total weight, tons

Total weight of potassium piping, renns:

\begin{tabular}{|c|c|c|c|}
\hline $\begin{array}{l}\text { Contactor } \\
\text { to Pump }\end{array}$ & $\begin{array}{l}\text { Pump to } \\
\text { Manifold }\end{array}$ & $\begin{array}{c}\text { Inlet } \\
\text { Manifold }\end{array}$ & $\begin{array}{l}\text { Manifold } \\
\text { to Modules }\end{array}$ \\
\hline 1832 & 1832 & 1832 & 1832 \\
\hline 0 & 30 & 30 & 30 \\
\hline 27 & 27 & 27 & 27 \\
\hline 3750 & 3750 & 1560 & 85.2 \\
\hline 1 & 1 & 8 & 16 \\
\hline 25 & 120 & 2 & 50 \\
\hline 48 & 48 & 36 & 6 \\
\hline 0.25 & 0.25 & 0.072 & \\
\hline 12.5 & 12.5 & 7.06 & 0.2 \\
\hline 320 & 1520 & 1730 & 78.5 \\
\hline 1.8 & 8.4 & 0.15 & 0.15 \\
\hline 0.1 & 0.2 & 0.1 & 1 \\
\hline 2 & 2 & 4 & 176 \\
\hline 3 & 16 & 60 & 26 \\
\hline
\end{tabular}

Location

\begin{tabular}{|c|c|c|c|}
\hline $\begin{array}{c}\text { Boiler to } \\
\text { Turbine }\end{array}$ & $\begin{array}{l}\text { Turbine to } \\
\text { Condenser }\end{array}$ & $\begin{array}{l}\text { Condenuer } \\
\text { to Pump }\end{array}$ & $\begin{array}{c}\text { Pump to } \\
\text { Boiver }\end{array}$ \\
\hline 1800 & 1100 & 1100 & 1700 \\
\hline 80 & 2.4 & 2.4 & 80 \\
\hline 0.15 & 0.0057 & 43 & 43 \\
\hline 1072 & 1072 & 1072 & 1072 \\
\hline 300 & 1200 & 15 & 15 \\
\hline 50 & - & 20 & 60 \\
\hline 5.5 & 14 & 1.5 & 1.5 \\
\hline 1.0 & 0.42 & 0.035 & 0.25 \\
\hline 23.75 & 154 & 1.75 & 1.75 \\
\hline 863 & - & 95 & 246 \\
\hline 20 & & - & 1.5 \\
\hline 0.5 & - & 0.5 & 1.0 \\
\hline 4 & 4 & 4 & 4 \\
\hline 80 & - & - & 6 \\
\hline
\end{tabular}

Niobium

80

s.s.

6 


\section{APRENDIX C*}

\section{EFFECTS OF THE MAGNETIC FIELD ON STAGNANT LQUID METAL}

Section 4.5.4 describes in general terms the effects of induced current densities in the lithium coolant and the pressures which result from $\overrightarrow{\mathbf{J}} \times \overrightarrow{\mathrm{B}}$ interactions. The following is a detailed analysis of the compression of lithium during the rise and fall of the compression field. Since the lithium is contained in ducts parallel to the field (coolant ducts) and perpendicular to the field, each case is discussed separately. Once the forces acting on the fluid are estimated, the effects of the resulting pressures on the flow patterns and structural stresses are estimated.

\section{C.1 EFFECTS ON FLUID IN FLOW CHANNELS PAR- ALLEL TO THE FIELD}

\section{C.1.1 Model}

A model for the flow channels is chosen to yield a simple yet accurate calculation of the induced current. ohmic loss, and pressure in the fluid in the various ducts in a time-varying magnetic field. The duct is assumed to have rectangular dimensions $L_{1}$ by $L_{2}, L_{1}>L_{2}$, as shown in Fig. $C-1$. The actual du ts are trapezoidal because of the cylindircal geometry but the effect on the numerical results of using the average rectangular dimensions will be small. The magnetic flux density is assumed to have no spatial dependence within the flow chansel. This is true for the applied magnetic field but is nc $r$ valid for the total field because the currents in the fluid set up an added induced field. The procedure followed is to assume no

-E. Pierson, ANI.

\section{Q $\mathrm{B}$}

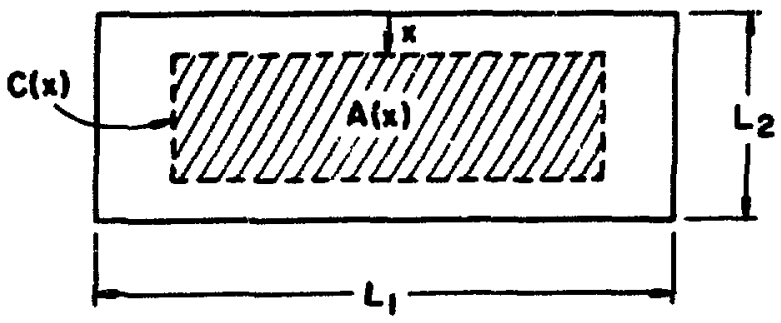

Fis. C.I. Geomerny used in parallel flow model. spatial dependence of the magnetio field, then calculate the currents and the induced field, and check the assumption. Numerical results show that this assumption is reasonable except near the beginning of the rise in the field where the terms of interest are all small. The fluid velocity along the chanuel, i.e., parallel to the magnetic field. has no influence on the terms calculated bere.

The current loops in the flow channels are assumed to follow the rectangular duct shape in Fig. C-1. This shape is chosen as the best model short of solving the boundary problem exactly, which would be a complex problem because of the induced electric field. The actual current paths are expected to be more rounded, approaching an ellipse or circle near the center of the channel. The model becomes more exact as $L_{1} / L_{2}$ increases.

\section{C.1.2 Analysis}

To determine the induced current in the fluid, consider the model of Fig. C-1. The electric field around the contour is

$$
E=\frac{A(x)}{C(x)} \frac{d B}{d t} \text {. }
$$

where $C(x)$ is the circumference of the contour, $A(x)$ is the area enclosed, and $x$ is the distance from the wall to the contour, $0<x<L_{2} / 2$. The current density, $\vec{J}=0 \vec{E}$, varies from zero at the center of the channel to a maximum at the edge, the maximum given by.

$\frac{\sigma L_{1} L_{2}}{2\left(L_{1}+L_{2}\right)} \frac{d B}{d t}$

The complete expression is

$$
\begin{aligned}
J(x)= & \frac{g}{2} \frac{d B}{d t}\left[\frac{\left[L_{1} L_{2}-\left(L_{1}+L_{2}\right)+4 x^{2}\right]}{\left(L_{1}+L_{2}-4 x\right)}\right] \\
& \left(A m / m^{2}\right) . \quad(C-2)
\end{aligned}
$$


The total current flowing in a channel, i.e., the current crossing a surface from the center of the channel to one wall. is

$$
\begin{array}{r}
I=\frac{\sigma}{32} \frac{d B}{d t}\left[2 I_{1} L_{2}+\left(L_{1}-L_{2}\right)^{2} \ln \left(\frac{L_{1}-L_{2}}{L_{1}+L_{2}}\right)\right] \\
(a m p / m) . \\
\text { (c-3) }
\end{array}
$$

This is the fluid current per meter of length parallel to the magnetic field.

The known current 1 can be used to calculate the change in the magnetic flux density between the center and the wall of the channel due to the current. This is $\Delta B=\mu_{0} I$, which is checked numerically to be sure that the assumption is valid.

The compression of the fluid is calculated for the known current density by means of the magnetic force density $\vec{J} \times \vec{B}$. The effect of the magnetic force is not the same as compressing the fluid with a piston. A piston creates a constant pressure throughout the fluid, whereas the magnetic body force makes the pressure vary from zero (or the statically applied pressure) at the wall to a maximum at the center of the channel. Since the variation of $\mathbf{J}$ across the channel is almost linear, becoming more linear as the ratio $L_{1} / L_{2}$ increases, the pressure variation is also almost linear. The exact expression for the total pressure difference between the center and wall is

$$
\begin{aligned}
\Delta p=\frac{\sigma B}{32} \frac{d B}{d t}\left[2 L_{1} L_{2}+\left(L_{1}-L_{2}\right)^{2} \ln \left(\frac{L_{1}-L_{2}}{L_{1}+L_{2}}\right)\right] \\
\left(N t / m^{2}\right) .
\end{aligned}
$$

The $\vec{J} \times \vec{B}$ force tends to compress the fluid toward the center of the channel (or, equivalently, to pull the fluid away from the channel walls). Note, however, that the fluid can move unimpeded along the magnetic field lines. The result may be thought of as being similar to squeezing a tube of toothpaste-, the fluid may tend to squirt out the ends. The distance that the fluid can move under this force is calculated from the integral along the channel of the momertum equation, $\rho l d v i d t=\Delta p$, where $\ell$ is the channel length parallel to the magnetic field, $\Delta p$ is the pressure difference causing the flow, and one end of the fluid is fixed by the blank wall. To integrate the equation for $\mathrm{dv} / \mathrm{dt}$ twice to yield the dispiacement $\Delta l$ it is necessary to know the magnetic field as a function of time.
Using the $\sin ^{2} \theta$ rise from an initial value of $B_{0}$ to 2 final value of $B_{p}$ yields

$$
\begin{aligned}
\Delta l= & {[2 \sigma / 5 \rho \ell]\left[2 \mathrm{~L}_{1} L_{2}+\left(L_{1}-L_{2}\right)^{2} \ln \left(\frac{L_{1}-L_{2}}{L_{1}+L_{2}}\right)\right] } \\
& {\left[\left(B_{P}-B_{0}\right)^{2}+4\left(B_{P}^{2}-B_{0}^{2}\right)\right] \Delta t(C-5) }
\end{aligned}
$$

where $\Delta t$ is the time interval of the field rise. This value for $\Delta l$ is high because we assume that there is no force outside of the channel opposed to the fiuid motion, and we neglect the problem of moving the fluid inward across the magnetic field lines so that it can squirt out the ends. This is considered later.

The compression $\delta$ of the fluid due to the magnetic field is determined from

$\delta=\int_{0}^{L_{2} / 2} K p d x$,

where $K$ is the compressibility and $p$ is the excess pressure over the static pressure due to the induced currents. The exact solution for $\delta$ is

$\delta_{e}=\left(\frac{\sigma L_{2}^{3}}{24} B \frac{d B}{d t}\right) \frac{3}{16 I_{2}^{3}}\left[-\left(L_{1}-L_{2}\right)^{3}\right.$

$\left.\operatorname{In}\left(\frac{I_{1}-L_{2}}{I_{1}+L_{2}}\right)-2 L_{2}\left(L_{1}^{2}-3 L_{1} L_{2}+\frac{2}{3} L_{2}^{2}\right)\right]$

A simplified approximate result obtained for $L_{1} \gg L_{2}$ is

$\delta_{a}=\frac{\sigma L_{2}^{3}}{24}$ B $\frac{d B}{d t}$

This represents a squeezing of the fluid away from the walls toward the center of the channel. The ratio $\delta_{\mathrm{e}} / \delta_{\mathrm{a}}$ is 0.706 for $L_{1} / L_{2}=2$ and increases as $L_{1} / L_{2}$ increases. 


\section{C1.3 Numerical Results}

To determine the magnitude of the effect of the rise in the compression magnetic field it is necessary to substitute typical numbers into the equations. The material properties chosen are $\sigma=2.5 \times 10^{6} \mathrm{mho} / \mathrm{m}, \rho=434$ $\mathrm{kg} / \mathrm{m}^{3}$, and $K=1.3 \times 10^{10} \mathrm{~m}^{2} /$ newton for lithium at around $500^{\circ} \mathrm{C}$. The magnetic flux density is assumed to rise as $\sin ^{2} \theta$ from $1.38 \mathrm{~T}$ to $11 \mathrm{~T}$ in $0.0314 \mathrm{sec}$, or $(\mathrm{dB} / \mathrm{dt})_{\text {nax }}=481 \mathrm{~T} / \mathrm{sec}$. The resulting numbers for two flow cli..nnels are given in Table $C$ - 1 . The pressure difference, $\Delta p$, and compression, $\delta$, are pioportional to $(B \cdot d B / d t)$ and thus rise from zero to 2 maximum and retum to zero for the constant part of B as shown in Fig. C-2. The upper limit to the fluid motion, $\Delta l$ calculated for $l=2 \mathrm{~m}$, is large enough to be significant if there is no pressure to oppose the flow.

The induced magnetic field with the time dependence shown in Fig. C-2 is numerically small compased to the applied field except at the start of the rise. A more accurate statement is that the induced field causes the total field in the center of the channel to lag by up to about $0.09 \mathrm{~T}$ (for the inner channel) behind the field outside i.e., a skin effect. This would displace the curves very slightly to the right (i.e., later in time), but would not have a significant effect on the conclusions.

\section{C.2 EFFECTS ON THE FLUID IN THE FEED LINES PERPENDICULAR TO THE FIELD}

\section{C.2.1 Model}

The model for the magnetic effects on the fluid in the feed lines to the flow channels is considerably more

\section{TABLE C-1}

\section{NUMERICAL RESULTS FOR THE COMPRESSION FIELD-FLUID EFFECTS}

\begin{tabular}{|c|c|c|c|}
\hline Parameter & Units & $\begin{array}{l}\text { Inner } \\
\text { Channel }\end{array}$ & $\begin{array}{c}\text { Next } \\
\text { Channet }\end{array}$ \\
\hline$L_{1}$ & $\mathrm{~cm}$ & 3.24 & 4.5 \\
\hline $\mathbf{L}_{2}$ & $\mathbf{c m}$ & 3.0 & 3.65 \\
\hline$\Delta p / l_{c}$ (peak) & $\begin{array}{l}\text { kPa } \\
\text { (Dsi) }\end{array}$ & $\begin{array}{l}539.3 \\
(77.6)\end{array}$ & $\begin{array}{l}873.5 \\
(125.7)\end{array}$ \\
\hline$\delta_{e}$ (peak) & $\mathrm{mm}$ & $0.681 \sigma^{3}$ & $1.3510^{3}$ \\
\hline$\Delta B$ (peak) & tesla & $910.010^{-4}$ & $1470.010^{4}$ \\
\hline$\Delta \boldsymbol{l}$ & cm & 19.4 & 31.5 \\
\hline
\end{tabular}
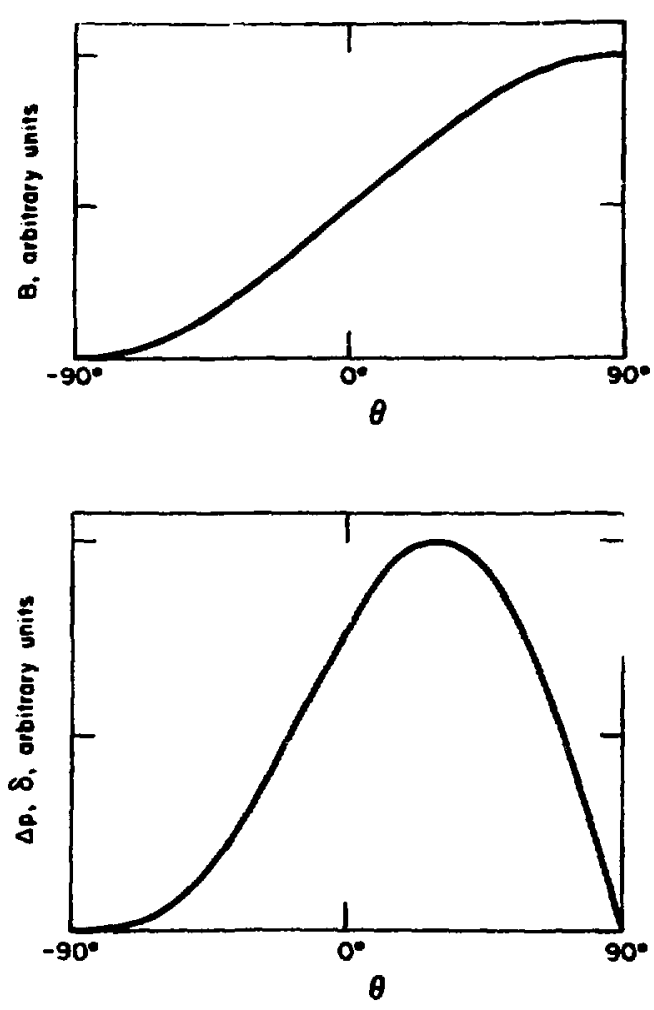

Fig. C.2.

Time-dependence of compression field, surface displacement, and radial pressure difference for a reccangular duct of litbium subjected to an axial magnetic field.

complicated for several reasons. Chief among these are that the magnetic field has a spatial dependence, the walls may act to short out current paths, and the calculations depend on the piping system extemal to the blanket. There are two effects to consider: the induced currents in the fluid and the resulting pressure and compression, and the possible motions due to the clectromagnetic forces. The fluid can move freely into or out of the flow channels along the field lines (part of the toothpaste effect of Sec. C.1.2, and there may be some motion across the field lines due to the large pressure differences.

To calculate the induced currencs and force in a feed line, a simplified geometry is assumed and attention is confined to the line feeding the innermost flow channel. The quasi-rectangular duct shown in Fig. C-3 has a length $L_{3}$ representing the distance from the plasma wall to the inner edge of the compression coil, and a depth $L_{4}$ representing the average depth of the trapezoid. The 


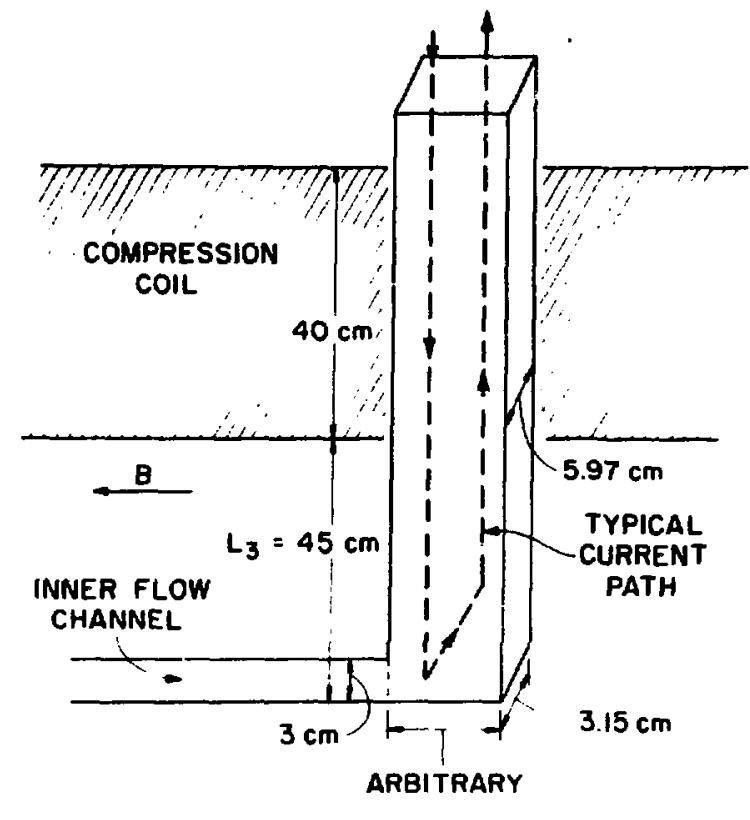

Fig. C-3.

Geometry used in transverse flow model.

dimensions indicated there correspond to an inner radius of $50 \mathrm{~cm}, 100$ segments in a circumference, and $45 \mathrm{~cm}$ from the plasma wall to the coil. To obtain an order of magnitude result, both the flux contribution and the resistance to current flow of the region going through the compression coil are neglected, and the resistance of the top leg of the current path is assumed negligible. The latter assumption is made because the current paths depend on the geometry outside of the field structure and the shorting of the duct walls. Neither effect occurs with the flow channels. This resistance will be small compared to the other legs in any case. The line width parallel to the magnetic field is arbitrary; it does not enter into the calculations. Once the currents are calculated, the pressure is determined as in Sec. C.1.2. Note that now there is no magnetic restraining force across the top of the duct because the current paths are closed outside of the field region. Thus the unbalanced magnetic force will cause some flow out of the line.

The flow and pressure couplings between the flow channel and the feed line are important and complex. For both, the induced currents cause the pressure to $b:$ higher in the center than at the walls. The pressure along the bottom and side walls of both is the static pressure of the system. The pressures in the centers are several hundreds of psi higher and different.

The flow patterns created may be strange. For example, consider the outer wall of the inner flow channel, Fig. C-3. From the electromagnetic force in the channel, the fluid along this wall should be at the static pressure, yet the pressure in the feed line just outside of that point will be hundreds of psi above static pressure. Thus, fluid may flow from the feed line into the cooling channel along this wall; this flow might compensate for any compression or toothpaste effect in the fluid in the channel, at least along the top wail. The electromagnetic pressure in the line is generally higher than in the channel, so that flow out of the channel is not expected.

\section{C.2.2 Analysis}

The model developed is already very approximate because of the assumptions regarding the closing of the current loops and the treatment of the region under the compression coil. Thus it is appropriate to make one further approximation and use the equations from Sec. C.1.2 for the case $L_{1} \gg L_{2}$. This is consistent because neglecting the resistance required to close the current loops is equivalent to $L_{1} \gg i_{2}$. For this case, the dimensions are $L_{3}=45 \mathrm{~cm}$, and $L_{4}=4.56 \mathrm{~cm}$, i.e., the approximation is very good. The relevant equations, with $L_{1}$ and $L_{2}$ replaced by $L_{3}$ and $L_{4}$, respectively, are

$$
I_{\ell}=\frac{\sigma}{8} L_{4}^{2} \frac{d B}{d t}(A / m)
$$

for the total fluid current, and

$$
\Delta \mathrm{p}_{\mathrm{Q}}=\frac{\sigma}{\mathrm{B}}
$$

for the pressure difference, where the subscript $l$ is used to denote a feed line quantity. The simplified expression for the compression of the fluid,

$$
\delta_{a L}=\frac{\sigma K L_{4}^{3} B}{24} \frac{d B}{d t}
$$

is used.

For the feed lines, the current ioops are assumed to close outside the magnetic field region, leaving an unbalanced force that will cause radially outward motion across the field lines. To calculate the motion of the fluid, the current density due to the fluid velocity, $v$, is found first. 
This is

$$
J_{m}=\sigma(\vec{E}+\vec{v} \times \vec{B}) \cong \sigma \vee B,
$$

where the electric field $\vec{E}$ required to close the current loops is neglected because of the difficulty in obtaining an exact solution relative to the increased accuracy. Note that $\vec{J}_{m}$ is in the opposite direction to the induced current density $\vec{J}$, and this means a decreased need for $E$ as they tend to cancel. Also, of course, the channel walls tend to short the return currents, and the stationary fluid will help close the $\vec{J}_{m}$ loops. Neglecting $\vec{E}$ means that the estimate for $v$ will be low because $E$ acts to decrease $\vec{J}_{m}$ for a given $v$, and hence decrease the force required to push the fluid across the field. The error is expected to be less than a factor of two.

If the fluid inertial and viscous forces are neglected, then the electromagnetic pressures due to the inducer! and motional currents must be equal, or

$v=\frac{\Delta \mathrm{P}_{\ell}}{\sigma \mathrm{B}^{2} \mathrm{~L}_{3}}=\frac{2 \mathrm{~L}_{4}}{8 \mathrm{BL} \mathrm{L}_{3}} \quad \frac{\mathrm{dE}}{\mathrm{dt}}$

Here $v$ is the maximum velocity which occurs only along the centerline of the feed line as the pressure difference decreases toward the wall. In using $\mathbf{L}_{3}$ as the length for the $J_{m}$ force, the small distance $L_{q}$ from the bottom wall required to reach full pressure difference is neglected. The expression for $v$ can be integrated directly to give the total distance moved as

$\Delta L_{\ell}=\frac{L_{4}^{2}}{8 L_{3}} \ln \frac{B_{C}}{B_{0}}$.

\section{C.2.3 Numerical Results}

Using the lithium properties as before, the above equations, and the dimensions $L_{3}=45 \mathrm{~cm}$ and the average $L_{4}=4.56 \mathrm{~cm}$, the resulting values are:

$\Delta P_{\ell}=2.349$ MPa peak (335 psi),

$\delta_{2} Q=0.018 \mathrm{~cm}$ peak,

$\Delta B_{\ell}=0.39$ Tesla peak.

$\Delta \ell_{\ell}=1.194 \mathrm{~cm}$.

At the con:lusion of the rise in the compression magnetic field, the currents and pressures become zero.

\section{C.3 EFFECTS ON THE FLOW CHANNELS}

\section{C.3.1 Model}

The rapid change in the magnetic flux density will cause induced currents in the closed paths formed by the metal flow channels, and these in turn cause ohmic loss and mechanical forces. The forces are significant because the magnetic compression of the lithium may leave room for the dust walls to deform, or the force may separate the inner conducting wall from the insulating layer which experiences no force of magnetic origin.

To calculate the induced currents, the flow channels are considered to be metal cans of circumference $C_{w}$, metal wall thickness $d$, and enclosed (flow) area $A_{w}$, where the subscript $w$ denotes a wall quantity. The actual channel shape is used here. The wall thickness $d$ is assumed to be small compared to the other dimensions so that the current density is constant over the thickness. All wall current is assumed to flow just in the wall, i.e. there is no shorting effeet due to the fluid. Each individual channel wall is assumed to be insulated from direct metalto-metal contact with the walls of the other channels. Finally, the induced current is assumed not to significantly affect the total flux density inside the flow channel; this assumption is checked by the numerical results.

\section{C.3.2 Analysis}

Theanalysis is as before, except that with the simpler geometry no integrals are required. The electric field around the wall circumference, $\mathbf{C}_{w}$, is

$E_{w}=\frac{A_{w}}{C_{w}}\left(\frac{d B}{d t}\right)$.

The total current per meter of length parallel to the magnetic field is

$I_{w}=\frac{\sigma_{w} A_{w} d}{C_{w}}\left(\frac{d B}{d t}\right)$.

The change in the magnetic flux density across the channel wall due to this (surface) current is

$\Delta B_{w}=\mu_{0} I_{w}=\frac{\mu_{0} \sigma_{w} A_{w} d}{C_{w}}\left(\frac{d B}{d t}\right)$. 
The total change in the flux density for a channel filled with a liquid metal suust also include the effect of the fluid current.

The force on the channel wall per unit area due to the current is

$F_{w}=\frac{\sigma_{w} A_{w} d B}{C_{w}}\left(\frac{d B}{d t}\right)\left(N t / m^{2}\right)$

This force tends to push in on or collapse the wall like an external pressure.

The ohmic energy loss in one cycle is

$$
w_{e w}=\frac{\sigma_{w}}{c_{w}} A_{w}^{2} \int\left(\frac{d B}{d t}\right)^{2} d t(J / m) .
$$

This is the energy loss per cycle in one channel wall per meter of length parallel to the magnetic field.

\section{C3.3 Numerical Results}

The numerical results for a wall conductivity of $2 \times 10^{6} \mathrm{mhos} / \mathrm{m}$, typical for niobium, and the dimensions for several channels are given in Table $\mathbf{C}$-2. All terms have the same time dependence as in Fig. C-2. Note that the peak force density may be substantial for a wall only 0.02 in. thick, and precautions must be taken. This is especially important for the wall around the graphire segments because it has the largest force. Cooling may also be necessary for the wall around the graphite.

\section{C4 SUMMARY AND CONCLUSIONS}

The most pronounced effect is the compression of the fluid in the flow channels, and the resulting possibilities

\section{TABLE C-2}

\section{NUMERICAL RESULTS FOR THE COMPRESSION FIELD-WALL EFFECTS}

\begin{tabular}{|c|c|c|c|c|}
\hline Purameter & Units & $\begin{array}{l}\text { Inner } \\
\text { Chennes }\end{array}$ & $\begin{array}{l}\text { Next } \\
\text { Chennel }\end{array}$ & Graphite cun \\
\hline d & $\mathrm{mm}$ & 0.1 & 0.1 & 0.1 \\
\hline & $\mathbf{m}^{2}$ & $9.73 \times 10^{4}$ & $16.4 \times 10^{4}$ & $69.7 \times 10^{4}$ \\
\hline & $\mathbf{m}$ & 0.125 & 0.183 & 0.389 \\
\hline & A/m & 3810.0 & 4921.0 & 8740.0 \\
\hline $\begin{array}{l}\Delta B_{w}(p=a k) \\
F \text { (penk) }\end{array}$ & $\begin{array}{l}\text { tesla } \\
\text { kPan }\end{array}$ & $\begin{array}{l}48.0 \times 10^{-} \\
28.4\end{array}$ & $\begin{array}{l}62.0 \times 10^{4} \\
36.6\end{array}$ & $\begin{array}{l}110.0 \times 10 \\
65.1\end{array}$ \\
\hline & (pii) & (4.08) & (3.27) & $(9.37)$ \\
\hline$w_{e}$ & $\mathrm{j} / \mathrm{m}$ & 23.5 & 51.3 & 387.0 \\
\hline
\end{tabular}

of no fluid pressure to counterbalance the magnetic force on the metal channel walls. The fluid compression is small, of the order of $0.4 \times 10^{-4} \mathrm{~cm}$, away from the walls. This can be compensated for by prestressing the fluid with a static pressure. The static pressure would cause the walls to bend outward with no magnetic pressure so as to not leave a gap with the magnetic pressure, but would require thicker walls and increase the possibility of leakage.

Special precautions may be required for the flow channel end walls and the feed line side walls as there the magnetic pressure adds to the static pressure since the fluid is free to flow along the field lines. The walls are probably not strong enough to withstand hundreds of psi unless additional support is provided or walls are placed back to back so that the net force on two adjacent walls is zero. Of course, the first wall facing the plasma may not be so supported.

Special care may be required to remove the energy loss in the conducting walls that are not cooled by the fluid. One example is the metal walls surrounding the graphite segments.

The flow patterns are the hardest to understand. The basic points are:

1. The magnetic field inhibits flow across the field lines. For example, the fluid in the feed lines is estimated to move only about $1.3 \mathrm{~mm}$ during the D-T bum pulse (less if inertial effects are included, as they must be).

2. The magnetic pressure in the flow channel, tending to cause flow out of the channel, is much smaller than the counteracring magnetic pressure in the feed line. In addition, there are special locations, such as the top edge of the flow channel, where the channel analysis predicts no magnetic pressure but the feed line analysis predicts a high magnetic pressure.

The estimated flow patterns, from this information, are:

1. There will be some loss of fluid from the feed line, but the total volume will be much less than a cubic inch.

2. The fluid loss from the feed line could allow a small amount of fluid to run out of the channel along the inner wall, but this is expected to be very, very small. (There is no magnetic pressure to cause this flow.)

3. There may be a very slight amount of circulation in the flow channel, but the channel should remain full of fluid. 


\section{APPENDAX D* \\ MECHANICAL AND ELECTRICAL ASPECTS OF A HOMOPOLAR MOTOR GENERATOR AS A TRANSFER CAPACITOR*}

\section{D.1 HOMOPOLAR MOTOR GENERATOR}

A homopolar motor generator can take any one of a number of forms. For simplicity, and because it is preferable for our purpose, our attention is limited to a modification of the form invented by Faraday in 1831. A enducting disk is mounted on bearings so that it can rotate around its axis in a uniform axial magnetic field, $B_{G}$. Actually, the field need not be uniform or purely axial but must be poloidal and symmetric about the plane of the disk; making $B_{G}$ uniform and axial simplifies the algebra. The magnetic field is separately excited by external coils. Brush contacts are made uniformly around the disk at the periphery and somewhere near the axis. The voltage generated between the brushes by rotation of the disk at a tangential velocity $v=r \omega$, is given by

$$
\begin{aligned}
v & =\int_{r_{1}}^{r_{2}} v B_{G} d r=\omega B \int_{r_{1}}^{r_{2}} r d r \\
& =\frac{3}{8} \omega_{G} B_{R^{2}}=\frac{3}{8} B_{G}^{R v} .
\end{aligned}
$$

where $\mathbf{R}$ is the radius of the disk or rotor. The inner brush contact is assumed made at half the radius of the disk. This gives three-fourths of the voltage that would be produced if the inner brush were at the axis but a large area is available for brush contact; the $\overrightarrow{\vec{J}} \times \overrightarrow{\mathrm{B}}$ shear forces applied to the rotor are limited, because the current has converged only half way to the axis.

The motor generator is assumed to have no shaft power transmitted to or from an external load or power source. Rotation results from current passed through the terminals, and any electrical energy generated is obtained by a decrease of mechanical energy of rotation. This means that the effective shaft size is the full diameter of the disk and that mechanical limitations on acceleration or deceleration are very generous.

\section{D.2 HOMOPOLAR CAPACITOR}

A separately excited homopolar machine behaves in a circuit as though it were a capacitor. This can be seen if it is recalled that the torque results from $\vec{J} \times \vec{B}$ forces. and therefore is proportional to the current. Assuming negligible friction, and remembering that the angular acceleration is proportional to the torque, then $\omega \sim i$. Assuming an initial angular speed of zero, and integrating in time,

$$
\omega \sim \int 13 T=Q \text {. }
$$

Since the voltage is proportional to the angular speed, i.e. $v \sim \omega, 2$ proportionality is seen to exist between the voltage and the charge. This is just the behavior of a capacitor. Other separately excited de machines also behave capacitively in circuits. We limit our discussions here to homopolar machines because of their inherently lower inductance, and because of their superior internal mechanical strength.

The capacity can be calculated explicitly by noting that the sapacitive energy stored is in reality the mechanical energy of rotation:

$$
\begin{aligned}
I / 2 \mathrm{Cv}^{2} & =1 / 2 I \omega^{2} \\
c & =\frac{I \omega^{2}}{v^{2}} .
\end{aligned}
$$

where $I$ is the moment of inertial of the disk.

\section{D.3 ROTOR SPEED AND MATERIAL}

The asgular speed of a rotor is limited by the maximum permissible stress generated by centrifugal forces. The tencike stress, $S$, in a radially thin hoop rotating around its sxis is given by

$$
s=p R^{2} \omega^{2}=p v^{2} .
$$

•J. Marshall, LASL. 
$S$ is measured in newtons $/ \mathrm{m}^{2}$ if $v$ is in $\mathrm{m} / \mathrm{sec}$ and $n$ is in $\mathrm{kg} / \mathrm{m}^{3}$. A reasonable stress in steel might be $3.745 \times 10^{8}$ $\mathrm{N} / \mathrm{m}^{2}\left(5 \times 10^{4} \mathrm{psi}\right)$. Taking the density of steel as $7.8 \times 10^{3}$ $\mathrm{kg} / \mathrm{m}^{3}$, Eq. (D-7) gives an allowable peripheral velocity for a steel hoop of $210.2 \mathrm{~m} / \mathrm{sec}$. A real flywheel is not normally made as a simple hoop, a solid cylindrical disk being much more common. The maximum stress in a cylindrical disk with an axial hole is given by

$$
\begin{gathered}
S_{\max }=\frac{\rho \omega^{2}}{4}\left[(3+\mu) b^{2}+\right. \\
\left.(1-\mu) a^{2}\right] .
\end{gathered}
$$

The quantities $a$ and $b$ are respectively, the radii of the hole and the disk, and $\mu$ is Poisson's ratio ( $\sim 0.3$ for most engineering metals). The maximum stress occurs at the periphery of the hole. For a solid disk (no hole). the maximum stress is then

$$
\begin{aligned}
S_{\max }= & \frac{3.3}{4} \rho v^{2}= \\
& 0.825 \rho v^{2}\left(N t / m^{2}\right) .
\end{aligned}
$$

Therefore, the maximum stress is $\mathbf{8 2 . 5 \%}$ of the stress in a thin hoop at the same peripheral velocity and the allowable peripheral speed is given by

$v=\sqrt{\frac{S_{\max }}{0.825 \rho}}(\mathrm{m} / \mathrm{sec})$.

For steel of strength $3.445 \times 10^{8} \mathrm{~N} / \mathrm{m}^{2}$. Fq. (D-10) gives an allowable peripheral speed for a solid disk of $v_{\max }=\$ 31.4 \mathrm{~m} / \mathrm{sec}$. Since the permissible peripheral speed varies inversely as the square root of the density, considerable advantage exists in making a homopolar rotor of a light metal. The highest speed thus attainable results in larger voltage, and therefore in smaller current at a given power level. Since homopolar machines are limited by brush current problems, low density and high peripheral speed may be advantageous.

In Fig. D-1 the peripheral speed is piotred as a function of allowable tensile stress for a number of metals; the highest speed is attained with an aluminum alioy having a yield point of $4.8 \times 10^{8} \mathrm{~N} / \mathrm{m}^{2}\left(7 \times 10^{4}\right.$ psi). Perhaps a real disk could be operated at half this stress. which would be reached at a peripheral speed of $\sim 330 \mathrm{~m} / \mathrm{sec}$.

If a machine is to be operated at field strengths near the saturation of steet, there might be an advantage in

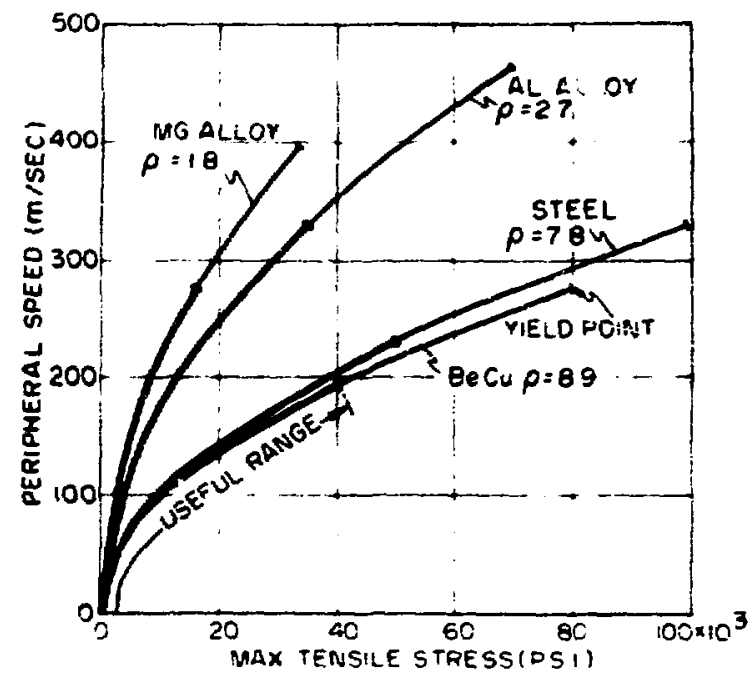

Fig. D.I.

Peripberal speed vs maximum tensile strengtb in a solid disk rotor. Curoes are extended only to approximate yield strengtb. Prrmissible speed ranges up to balf tbe yield stress are shown as beury lines.

using a steel disk. If the magnecic circuit is nearly all ferromagnetic, a valuable enhancement of the field strength results. This is true even at field strengths presently attainable with superconducting coils in large magnets. An additional advantage of steel is that it is less likely to be corroded by liquid metal brush materials than by some of the other possibilities. A disadvantage is that there will be ferromagnetic forces on the bearings.

\section{D.4 THE USE OF HOMOPOLAR CAPACITOR}

The optimum use of a homopolar capacitor would be simply as an energy storage device. It would be spun up or charged as a homopolar motor from a charging supply, and then discharged into a load. This introduces most of the problems of homopolar rotating machinery associated with high-current brushes. An alternative is to use the homopolar machine as a transfer capacitor between an inductive energy store and an inductive load. Initially the rotor would be at rest with the field energized. During energy transfer it would be spun up and spun down again within a few milliseconds. Since rotation would persist for only a very short time, the brushes ind bearings could be miuch more frictional than would be allowable in a continuuusly running machine. The brushes might conceivably 
be simple wiping metal contacts or the disk might be completely flooded with a liquid metal such as mercury or NaK. The serious problems of switching and current connections to the homopolar capacitor from a superconducting inductive energy store are ignored for the present.

\section{D.S SPECIFIC DESIGN PROFOSAZ}

The proposed design uses two counterrotating disks connected in series. The disks will be $50 \mathrm{~cm}$ in radius, $5 \mathrm{~cm}$ thick, and completely immersed in a liquid metal for brush contact. A conceptual design of the unit is shown in Fig. D-2. Arrows indicate current paths. The return current for disk \#1 flows outward on stator \#1 from the top side of the disk and on disk \#2 from the bottom side. The reaction to the torque on the top side of disk \#1 is applied to stator \#1. The reaction to the torque on the bottom side is applied to the top side of disk \#2. The torques on the two rotating disks are equal and opposite so that no net torque is applied to the foundations. The large torque between stator \#1 and stator \#2 requires the frame of the machine to be strong enough to support it.

the disks are made of high tensile strength steel. Steel is less likely to be corroded by NaK or mercury than aluminum alloy. Otherwise, aluminum wouid probably be preferable. Sheets of insulation are inserted between the two disks and between disks and stators to prevent short circuiting of the current by the liquid metal brush material. Contact is made to the outside of the disks and from disk to stator and disk to disk by liquid metal. Complete

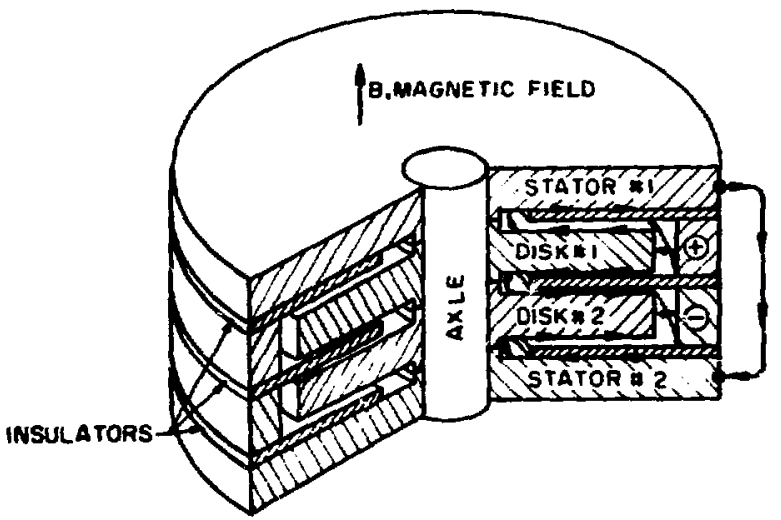

Fig. $D \cdot 2$.

Conceptual design of a two-disk hrmopolar capacitor. All volume surrounding disks to be flooded with liquid metal ( $\mathrm{Hg}$, NaK). flooding with liquid metal, possibly pressurized, prevents interference with brush contact by hydrodynamic and magnetsc forces. Liquid metal tends to short circuit the radial surfaces of the disks on which current must flow to apply $\vec{j} \times \overrightarrow{\mathrm{B}}$ torque. This will make the brush liquid rotate so as to produce a back emf which will oppose the current. This should allow torque to be applied to the disks. The layers of liquid metal would be kept thin in any case. Any number of two disk units could be stacked on top of each other and connected in series with sheets of insulation between them. The magnetic field could be supplied by a long solenoid.

A set of sample calculations is reproduced here for each disk assuming $50 \mathrm{kG}$ (5 tesla) axial field. Energy transfer occurs in 2 msec ( $1 \mathrm{~m}$ sec to spin disk up to full velocity and another to spin it down again). The disks are assumed to nun safely at a peripheral speed of $230 \mathrm{~m} / \mathrm{sec}$ $(\omega=460 \mathrm{radians} / \mathrm{sec}$ ). Brushes are at $50 \mathrm{~cm}$ and $25 \mathrm{~cm}$ radii.

- Maximum voltage limited by allowable angular velocity of rotor

$$
\begin{aligned}
& v=\int^{r_{2}} v B d r=w B \int^{0.5} r d r \\
& r_{1} \quad 0.25 \\
& =460 \times 5\left[\frac{x^{2}}{2}\right]_{0.25}^{0.5}=215.6 v(D-8) \\
& m=\pi r^{2} \ell \rho=\pi \times 0.5^{2} \times .05 \times 7.8 \\
& \times 10^{3}=306.3 \mathrm{~kg}
\end{aligned}
$$

- Moment of inertia

$I=\frac{I}{2} \mathrm{~m} \mathrm{r}^{2}=38.29 \mathrm{~kg} \mathrm{\textrm {m } ^ { 2 }}$

- Kinetic energy

$$
\begin{aligned}
U & =\frac{1}{2} I \omega^{2}=\frac{38.29}{2} \times 460^{2} \\
& =4.051 \times 10^{6} \mathrm{~J}
\end{aligned}
$$


- Maximum torque. The maximum acceleration experienced in spinning up sinusoidally in 1 mses is the same as uniform acceleration to spin up in $2 / \pi$ msec or in 637 $\boldsymbol{\mu}$ sec.

$$
\begin{aligned}
& \text { Torque }=\theta=I \stackrel{0}{\max }^{\circ}=38.29 \\
& \times \frac{460}{637 \times 10^{-6}}=2.765 \times 10^{7} \mathrm{~km}
\end{aligned}
$$

- Electrical torque

$$
\begin{aligned}
\theta & =\int_{r_{1}}^{r_{2}} 1 \mathrm{~B} r \mathrm{dr}=51\left|\frac{r^{2}}{2}\right|_{0.25}^{0.5} \\
& =0.468751
\end{aligned}
$$

so

$$
\begin{aligned}
1 & =\frac{\theta}{.46875}=\frac{2.765 \times 10^{7}}{.46875} \\
& =5.898 \times 10^{7} \mathrm{~A}
\end{aligned}
$$

- Maximum $\vec{j} \times \vec{B}$ shear force. The maximum shear force on rotor surface occurs at the inner brush where radial current has its maximum surface density.

$$
\begin{aligned}
&(j \times B)_{\max }=\frac{5.898 \times 10^{7}}{2 \pi \times 0.25} \times 5 \\
&=1.8774 \times 10^{2} \mathrm{Nm}^{2} \\
&(\mathrm{D}-15)
\end{aligned}
$$

This is applied to both surfaces of the disk. Shear force on one surface is half as much. Hence,

$$
\begin{aligned}
& S=\text { Shear force }=0.9387 \\
& \times 10^{8} \mathrm{~km}^{2}\left(1.36 \times 10^{4} \mathrm{psi}\right)
\end{aligned}
$$

The maximum shear force, then, is well within the sirength of the steel.

The above calculations are for one disk. Two disks in serie: will produce twice the voltage, store twice the energy, and have half the capacity. The unit then acts like a capacitor of

$c=\frac{I \omega^{2}}{v^{2}} \times \frac{1}{2}=87.13 \mathrm{~F} \quad(\mathrm{D}-17)$

The maximum voltage is $231 \mathrm{~V}$ and stored energy is 8.10 MJ. 